\title{
Antenatal inflammatory insults and preterm brain injury
}

Citation for published version (APA):

Gussenhoven, R. (2019). Antenatal inflammatory insults and preterm brain injury: Pathophysiology and therapeutic strategies. [Doctoral Thesis, Maastricht University]. https://doi.org/10.26481/dis.20191216rg

Document status and date:

Published: 01/01/2019

DOI:

10.26481/dis.20191216rg

Document Version:

Publisher's PDF, also known as Version of record

\section{Please check the document version of this publication:}

- A submitted manuscript is the version of the article upon submission and before peer-review. There can be important differences between the submitted version and the official published version of record.

People interested in the research are advised to contact the author for the final version of the publication, or visit the DOI to the publisher's website.

- The final author version and the galley proof are versions of the publication after peer review.

- The final published version features the final layout of the paper including the volume, issue and page numbers.

Link to publication

\footnotetext{
General rights rights.

- You may freely distribute the URL identifying the publication in the public portal. please follow below link for the End User Agreement:

www.umlib.nl/taverne-license

Take down policy

If you believe that this document breaches copyright please contact us at:

repository@maastrichtuniversity.nl

providing details and we will investigate your claim.
}

Copyright and moral rights for the publications made accessible in the public portal are retained by the authors and/or other copyright owners and it is a condition of accessing publications that users recognise and abide by the legal requirements associated with these

- Users may download and print one copy of any publication from the public portal for the purpose of private study or research.

- You may not further distribute the material or use it for any profit-making activity or commercial gain

If the publication is distributed under the terms of Article $25 \mathrm{fa}$ of the Dutch Copyright Act, indicated by the "Taverne" license above, 


\title{
Antenatal inflammatory insults and preterm brain injury:
}

\section{Pathophysiology and therapeutic strategies}

\author{
PROEFSCHRIFT
}

ter verkrijging van de graad van doctor aan de Universiteit Maastricht, op gezag van Rector Magnificius, Prof. dr. Rianne M. Letschert, volgens het besluit van het college van Decanen, in het openbaar te verdedigen op maandag 16 december 2019 om 14.00 uur

door

Ruth Gussenhoven 


\section{"The brain is still a world}

consisting of a number

of unexplored continents

and great stretches of

unknown territory."

- Ramon y Cajal
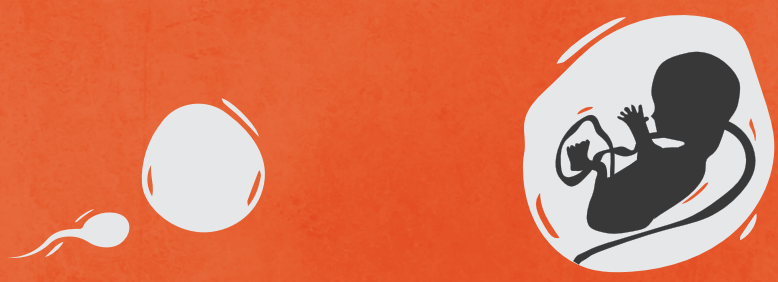

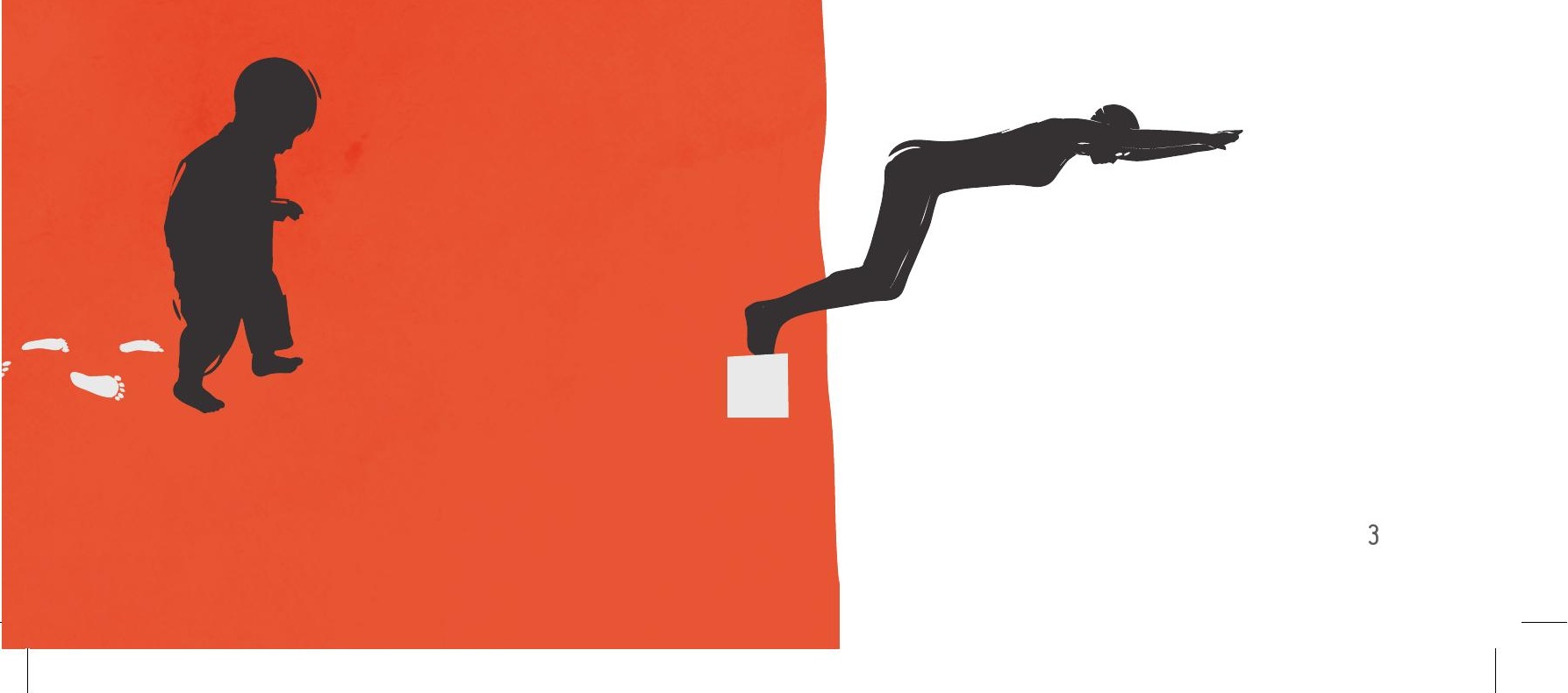


\section{Promotores}

Prof. Dr. Boris W. Kramer

Prof. Dr. Luc J.I. Zimmermann

Dr. Tim G.A.M. Wolfs (ius promovendi)

\section{Assessment committee}

Prof. Dr. R. Jeroen Vermeulen (chair)

Prof. Dr. Marc E.A. Spaanderman

Dr. Daniel L.A. van den Hove

Prof. Dr. Freek E. Hoebeek - UMC Utrecht

Prof. Dr. Jane Pillow - University of

Western Australia 


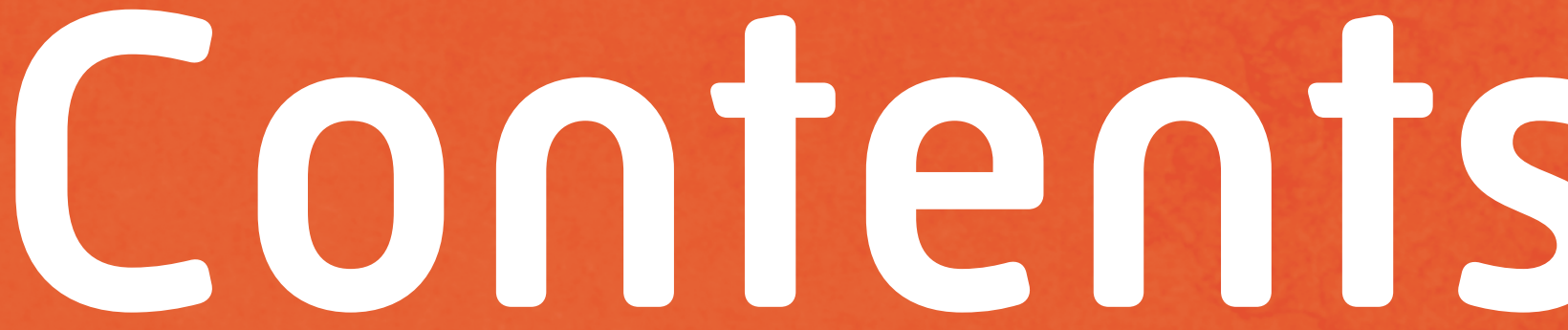

1. General introduction

2. The paradoxical effects of chronic intra-amniotic Ureaplasma 34 parvum exposure on ovine fetal brain development

3. Chorioamnionitis, neuroinflammation and injury; timing is key in the preterm ovine fetus

4. Neuroinflammation and structural injury of the fetal ovine brain following intra-amniotic Candida albicans exposure

5. Systemic Multipotent Adult Progenitor Cells protect the cerebellum after asphyxia in fetal sheep

6. Annexin A1 as neuroprotective determinant for blood-brain barrier integrity in neonatal hypoxic-ischemic encephalopathy

7. Summary \& general discussion

8. Nederlandse samenvatting

9. Valorization

10. Curriculum Vitae \& List of publications 


\section{How does the brain work?}

One of the greatest scientific questions of the last century and still one of the most fascinating mysteries on earth is "How does our brain work?" (Dick Swaab, author of Wij zijn ons Brein). Renowned philosophers are already trying for ages to understand whether the human brain can understand itself, however satisfactory answers remain unfound. Historically, the predominant perception of the brains functioning finds its origin in dualism which distinguishes the mind from the body. Nowadays, a shift into modern physicalism, stating that body and mind are physical, opened new paths in discovering the brains functioning. Neurologists and cognitive scientists have extensively contributed to the contemporary knowledge in brain research which has evolved enormously over the last decades. These researchers aim to mimic, describe and understand the brain's functioning. But maybe even more important is the dysfunctioning of the brain what we are trying to understand so badly. What happens in the brain during disease, how the behavior of the cells changes, and which processes are involved in this, is the driving force behind the research of the present brain pathophysiology.

The continuing search of desperately trying to discover where brain pathologies originate from raises another important concept that was critically discussed over the last years, namely Barker's hypothesis. This fetal origin of adult diseases is nowadays well accepted, and more commonly referred to as DoHAD (Developmental Origins of Health and Disease) in which Barker proposes that many challenges in life find their origin in susceptibility before birth. The fact that neurocognitive dysfunctioning in some, or maybe even the majority of cases, starts already very early in life or even during early phases of embryology before we call it life, is an interesting thought and increases the importance of research into the field of fetal brain (organ) development. The womb that houses the child before birth is supposed to be a safe and protective environment that secures normal development; however, many environmental influences from the outside might affect this developmental trajectory with potential long-term adverse consequences. And these environmental influences already start 
as soon as we have an environment which means as soon as they are implanted in the womb, or possibly already during preconception. This is also elegantly displayed by the interesting documentary In utero by Kathleen Gyllenhaal that summarizes and discusses the influence of experiences in the womb on the health of people and society from a scientific, economic and historical point of view. This fetal origin hypothesis also points at the potential need to intervene earlier as we are used to, to secure normal and healthy development throughout life. Therefore, studying the brain during its most critical period of development is crucial for the development of new neurotherapeutics to protect the brain and ensure a healthy life.

Modern research, equipped with state of the art analytical techniques including microscopy, magnetic resonance imaging (MRI) and mass spectrometry, enables scientists to explore the brain with increasing detail. Yet we have to keep in mind that our knowledge of the brain is still overshadowed by what we do not know: "The brain is still a world consisting of a number of unexplored continents and great stretches of unknown territory." (Ramon y Cajal). Nevertheless, as today we are able to look back on the road and progress that has been made over the past years, all little steps add up and together it has covered quite some distance. And for the future we will keep going forward, step by step as "One may walk over the highest mountain one step at a time". 


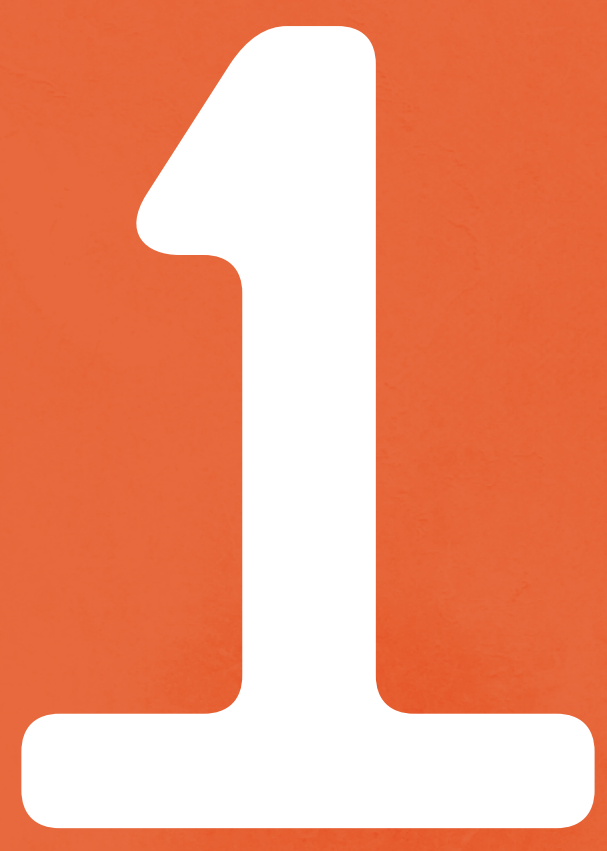

General

introduction 



\section{Encephalopathy of prematurity}

Human brain development is a protracted process that starts already very early in utero around the 3th week of gestation with the differentiation of the neural progenitor cells [1]. It further develops during pregnancy, being in the proposed safe and protective environment of the womb, until 40 weeks of full term pregnancy are completed. Moreover, even after birth brain development continues further for many years. Especially during the last half of gestation, a spectacular period of brain growth and development occurs in which the brain weight increases with $90 \%$ and evolves from a smooth surface area (lissencephaly) to a surface consisting of numerous gyri and sulci (gyrencephaly) [2]. Especially, in utero, during this important critical period of brain development, even mild disturbances can alter the trajectory of development leading to disproportionately adverse brain functioning. Premature birth, defined as birth before 37 weeks of completed pregnancy, is the most important interference of brain development that might result in long lasting neurodevelopmental disabilities [3, 4]. Unfortunately, preterm birth continues to be a major global health problem comprising $11.1 \%$ of all livebirths around the world in 2010 (14.9 million babies born before 37 weeks of gestation) resulting in an enormous social and economic burden to patients, families and society [5, 6]. Preterm birth is the leading cause of death of babies under 5 years of age [7]. In survivors, preterm birth can result in a variety of long-term complications with the frequency and severity of adverse outcomes rising with decreasing gestational age and decreasing quality of care [8]. In around 50-60\% of infants born extremely preterm ( $<27$ weeks of gestation), cognitive and behavioral impairments will develop [9].

Preterm brain injury, or as it nowadays is referred to as Encephalopathy of Prematurity (EoP) [9], is a syndrome of overall brain dysfunction as a consequence of the complex and multifactorial grey and white matter lesions in the cerebrum and cerebellum following preterm birth reflecting acquired insults, altered developmental pathways and reparative phenomena. These acquired insults include hypoxia-ischemia, maternal-fetal infection, 
postnatal sepsis, drug- and toxicant exposures, pain, neonatal stress and malnutrition [10]. Accordingly, these various factors can interact, thereby increasing or decreasing the damage, also referred to as sensitization or preconditioning [11]. Moreover, every preterm baby has its unique additional factors that might modify the cerebral outcome or trigger preterm birth including gender, genetics, epigenetics and socioeconomic status [12]. Considering the resulting heterogeneity of the lesions comprising EoP, the clinical spectrum of neurodevelopmental abnormalities in survivors of preterm birth is wide. Combinations of deficits in cognitive, behavioral and attention functions that manifests as social limitations and restrictions, school performance and language impairments, and mild to severe motoric disabilities like cerebral palsy occur during childhood and even up to adolescence $[2,3]$.

Among the multiple etiological factors contributing to EoP, there are two key factors that I will highlight here since they are clinically the most common and significant contributing factors to adverse fetal brain development. These factors are intrauterine infection/inflammation (i.e. chorioamnionitis or triple-I) and global hypoxia-ischemia and these comprise the main focus of the research described in this thesis.

\section{Intrauterine infections and/or inflammation}

Chorioamnionitis (i.e. antenatal infection/inflammation) is defined as inflammation of the fetal membranes (chorion and amnion), histologically characterized by diffuse infiltration of neutrophils into these membranes. Chorioamnionitis is the most common cause of preterm birth (25-40\%) and incidences are increasing with decreasing gestation age [13]. Chorioamnionitis is only present in 3-5\% of deliveries at term; however, in deliveries between 21-24 weeks of gestation in $94 \%$ of the placentas chorioamnionitis has been diagnosed [14]. Chorioamnionitis is considered to be a polymicrobial syndrome including Mycoplasmas species (Ureaplasma species in particular), Gardnerella vaginalis and Fusobacteria species as the most common isolated bacteria, but viral and fungal species can also be found [15]. Especially women who became pregnant with intrauterine contraceptive devices are at high risk for intra-amniotic infection with Candida albicans [16]. Although incidences of intrauterine $C$. albicans infections are low, the fetal consequences are severe. An intrauterine C. albicans infection has a high fetal mortality rate and can result in fetal candidiasis with severe adverse outcomes including impaired neurodevelopment [17, 18]. Besides 
contamination via intrauterine contraceptive devices, other routes in which microorganisms can invade the amniotic cavity are ascending from the lower genital tract (most common), hematogenous through the placenta, accidental introduction via invasive procedures (i.e. amniocentesis / percutaneous umbilical cord blood sampling) and retrograde spread via fallopian tubes [19]. Since the ascending route is the most important infectious cause, it is not surprising that microbial invasion of the amniotic cavity is more frequently found in pregnancies with preterm premature rupture of the membranes (PPROM) and longer duration of labor. However also intact membranes can be invaded by microbes and cervical insufficiency, twin gestations, meconium-stained amniotic fluid, presence of genital tract pathogens (eg, sexually transmitted infections, group B Streptococcus, bacterial vaginosis) and idiopathic vaginal bleeding are also associated with increased risk of intra-amniotic infections.

Interestingly, not in all cases of inflammation of the fetal membranes a microbe is found/detected/isolated [14]. This 'sterile' intra-amniotic inflammation can be induced by other 'danger signals' that are released under conditions of cellular stress, injury or cell death [14]. Independent of the cause of inflammation (sterile vs. non-sterile) and also independent of the induction of preterm birth, chorioamnionitis is associated with adverse outcomes of fetal organ development including the brain [20]. In particular, chorioamnionitis is linked to an increased risk of intraventricular hemorrhage [21] and neurologic impairment/injury [22, 23] including cerebral palsy [24]. Therefore, it would be of clinical importance to diagnose such intra-amniotic infection/inflammation in order to intervene if possible and when necessary. However, assessment of the presence of an intraamniotic infection during pregnancy is hindered by the fact that most infections are subclinical in nature and occur in the absence of clinical (i.e. symptomatic) chorioamnionitis.

From an immunological point of view, pregnancy is an interesting phenomenon since the mother and fetus tolerate each other's presence. This maternal-fetal immunological tolerance allowing normal pregnancy might explain why microorganisms can overcome certain barriers and gain access to the uterine cavity. Additionally, it explains why the mother can tolerate the presence of these bacteria in her uterus for long periods without clinical manifestations. Accordingly, chorioamnionitis is mostly diagnosed after birth by histological examination of the placenta. When 
clinical symptoms do occur this includes a combination of fever, maternal-fetal tachycardia, uterine tenderness and/or foul-smelling amniotic fluid.

When the fetus is directly exposed to intrauterine inflammation/infection via direct skin contact or swallowing and breathing movements in utero, the fetal immune system responds via the release of pro-inflammatory cytokines inducing a fetal inflammatory response syndrome (FIRS) [13]. FIRS is characterized by elevation of fetal plasma interleukin-6 (IL-6), a pro-inflammatory cytokine involved in hematopoiesis, final maturation of antibody-producing plasma cells, T-cell activation and differentiation and secretion of acute phase proteins by the liver [25]. An elevation in fetal plasma IL-6 concentrations is an independent risk factor for the occurrence of severe neonatal morbidity including intraventricular hemorrhage (IVH) and periventricular leukomalacia (PVL) [26, 27]. Moreover, funisitis and chorionic vasculitis which are the clinical hallmarks of FIRS are associated with clinical chorioamnionitis, the onset of preterm labor, and a higher rate of multi-organ adverse outcomes including the brain $[13,28]$.

A recent study including 2,390 extremely preterm infants $(<27$ weeks of gestation) demonstrated that fetal exposure to histological and clinical chorioamnionitis was associated with an increased risk of cognitive impairment at 18-22 months of corrected age compared to infants exposed to no chorioamnionitis or histological chorioamnionitis alone [29]. These combined data propose that the release of inflammatory cytokines (FIRS) in the course of intrauterine infections may play a crucial role in the initiation of brain injury [27]. Fetal systemic inflammation is a known inducer of a cerebral inflammatory response and this route is considered to result in altered neurodevelopmental outcomes (see paragraph 1.2) [30].

\section{Global hypoxia-ischemia}

During pregnancy, the fetus merely depends on its mother for oxygen and blood supply via the umbilical cord and placenta. Therefore, fetal global hypoxiaischemia (HI) is the result of an impaired gas exchange between mother and fetus. Pre- and perinatal causes can be inadequate placental perfusion due to maternal complications (preeclampsia), impaired umbilical cord flow due to compression, rupture of the placenta or prolapse of the umbilical cord during pregnancy or labor. Postnatally, global HI can result from inadequate blood pressure (auto)regulation, ineffective ventilation/oxygenation and systemic 
infections. Brain injury as a result of hypoxic-ischemic event(s) is usually referred to as hypoxic-ischemic encephalopathy (HIE) which is a severe birth complication including high morbidity and mortality rates that occurs in 1-8 per 1000 live births [31]. Accordingly, HIE has detrimental effects on brain development with severe long-term disabilities including mental retardation, epilepsy and cerebral palsy. Moreover, incidences in preterm infants are higher than we previously thought indicating that HI plays a more important role in the pathophysiology of EoP, though compared to term infants, the hypoxicischemic event in preterm infants is usually less severe and more chronic in its course [32]. Classically, the organ injury caused by $\mathrm{HI}$ can be divided in three phases [33]. The initial phase (primary energy failure) results from a decreased supply of oxygen in which the tissue switches to anaerobic metabolism generating less adenosine triphosphate (ATP) and lactate. Due to this energy loss, membrane pumps will fail, resulting in accumulation of intracellular sodium and calcium and subsequent cytotoxic edema, release of excitatory neurotransmitters (especially glutamate) and free fatty acids accumulation from degrading cellular structures. Glutamate activates the N-methyl-D-aspartate (NMDA) and $\alpha$-amino-3-hydroxy-5-methyl-4-isoxazolepropionic acid (AMPA) receptors present on pre-oligodendrocytes and neurons resulting in reactive oxygen and nitrogen species (ROS and RNS) production. The secondary phase of injury (secondary energy failure; 6-72h after the insult) is mainly the result of restored reperfusion leading to induction of an inflammatory reaction in the brain, continuing synthesis of free radicals (ROS), mitochondrial dysfunction and persistent glutamate excitotoxicity that eventually results in cell death. Cell death mechanisms include apoptosis, autophagy and/or necrosis. Whereas apoptosis and autophagy are more controlled paths leading to cell death mediated by intracellular programs, necrosis is the result of external factors including infection or toxins resulting in premature cell death via unregulated degradation of cell components. These cell components function as danger associated molecular patterns (DAMPs) that trigger a cerebral inflammatory response that ultimately can lead to altered brain development and injury (phase III; chronic inflammation days to years following the insult) (see paragraph 1.2). 


\section{Pathophysiology of EoP}

Both antenatal infection/inflammation and global hypoxia-ischemia can result in encephalopathy of the newborn. The underlying pathophysiological mechanisms resulting from perinatal stress (i.e. inflammation or $\mathrm{HI}$ ) are multifactorial and complex. Among these numerous developmental processes occurring during brain development, some are related to cellular immaturities making them more vulnerable to inflammatory cytokines, glutamate and free radicals. Therefore, these processes are of particular interest in the preterm infant and play a crucial role in the pathophysiology of EoP. In this paragraph the pathophysiology of EoP is outlined which is based on strong clinical evidence and a large number of studies in animal models for intra-amniotic infections/inflammation and global hypoxia-ischemia.

\section{Cerebral inflammatory response - a disturbed balance}

Early-life immune challenges including intrauterine inflammation and $\mathrm{HI}$ can interfere with fetal brain development. Microglia and astrocytes are the most important immune cells of the brain involved in many crucial developmental processes and accordingly disturbed activation of these cells during early life have the potential to permanently disturb proper brain development. At the interface of the fetal peripheral blood and the brain, the blood-brain barrier (BBB), pro-inflammatory cytokines including IL-6 contribute to the activation of cerebral endothelium as part of the BBB. Subsequently, activation of adjacent microglia and astrocytes occurs as their primary targets and producers of inflammatory mediators, ultimately resulting in cerebral inflammatory response.

Microglia are the resident innate immune cells of the brain and function as "gate-keepers" ensuring a healthy microenvironment in the brain. Aberrant or excessive activation of microglia is linked to impaired neurogenesis and white matter development [34, 35]. Microglia exert multiple physiological functions that are crucial during healthy brain development. These functions include the secretion of trophic factors and phagocytosis which are important in synaptic pruning and development of connectivity 
pathways, oligodendrogenesis, vasculogenesis and vascular sprouting [36, 37]. Moreover, altered microglial functioning has been linked to autism spectrum disorder (ASD)-like behavior in mice and is proposed to be the result of disrupted microglia-mediated synaptic pruning [38]. When microglia are being exposed to different types of DAMPs and PAMPs they become activated/triggered and serve as antigen presenting cells [39-42]. Despite their different etiological factors (infectious or non-infectious), they all trigger acute and/or chronic inflammation and are proposed to share the same activation pathways leading to impaired white matter development and neural maturation and connectivity ultimately resulting in altered brain functions [43]. Downstream mediators that are involved in inflammationand/or HI-induced injury include the induction of immune mediators (cytokines and chemokines), reactive oxygen and nitrogen species (ROS and NOS), excitotoxicity (glutamate), mitochondrial impairment, and reduced vascular integrity [11].

Accordingly, this reduced vascular integrity also includes an increased blood-brain barrier (BBB) permeability. The BBB exerts an important role in the regulation of brain homeostasis by preventing potential harmful infiltrating immune cells and inflammatory molecules from entering the brain, removing waste products from the brain and regulating fluid and metabolic balance [44]. Disruption of the BBB is increasingly recognized as a pathophysiological mechanism of EoP [44, 45]. Pro-inflammatory cytokines that are released by activated immune cells during antenatal inflammation and/or HI both upregulate enzymes associated with the breakdown of the BBB. In line, increased permeability facilitates the influx of these blood-borne inflammatory cells (monocytes, neutrophils, T-lymphocytes) into the brain and thereby further exacerbating the cerebral inflammatory response.

Astrocytes are also indispensable for normal brain development and besides an essential component of the BBB its functions include regulation of the extracellular glutamate homeostasis, providing structural and metabolic support to surrounding cells (e.g. oligodendrocytes) and modulate neuronal connections [46]. Changes in astrocyte function as a result of perinatal inflammatory stress (i.e. infection or HI) have been implicated in altered neurological outcomes and are associated with increased risk of ASD and cerebral palsy $[47,48]$. 


\section{Diffuse white matter injury - the developing oligodendrocyte}

At present, the clinical hallmark of brain injury in preterm infants is diffuse white matter injury (dWMI) [10]. For decades cystic periventricular WMI or leukomalacia (PVL), referring to the large cystic lesions in the deep periventricular white matter, was the most common type of white matter injury observed in preterm babies. Fortunately, this type of white matterinjury has almost disappeared due to improved obstetrical and neonatal healthcare. Nevertheless, more diffuse types of white matter injury are increasingly recognized[10]. This diffuse WMI is clinically characterized by diffuse microscopic, punctate lesions, decreased white matter volumes and thinning of the white matter tracts $[49,50]$.

White matter consists of myelinated axons that allow proper brain connectivity by enabling rapid action potential transmission and providing axonal protection. Oligodendrocytes (OLs) are the cells responsible for myelin production, especially during the third trimester of pregnancy OLs undergo critical phases of development that make these cells extra vulnerable for perinatal insults [51]. OLs originate from neural stem cell (NSC)-derived oligodendrocyte precursor cells (OPCs) that differentiate into immature premyelinating OLs (pre-OLs) and finally differentiate into mature OLs that, when these pre-OLs come in contact with axons, start producing myelin [51]. Particularly the pre-OLs, that are excessively present during 24-30 weeks of gestation, are the key cellular target in EoP [52]. Pre-OLs exhibit maturation-dependent characteristics that render them more vulnerable to injurious hits including their overexpression of excitatory amino acid receptors and immature anti-oxidant system [53, 54]. Under normal conditions, a consistent pool of OPCs is maintained by continuous proliferation of OPCs. During development, OPCs migrate from the subventricular zone into white matter regions, where they stop dividing and differentiate into mature myelinating OLs. If this balance is disturbed due to inflammatory / hypoxic insults, pre-OL decrease in number and local OPCs are triggered to proliferate and replenish pre-OLs to maintain a balanced number of OPCs. However, these pre-OLs fail to differentiate into myelin-producing cells since signals that promote proliferation will inhibit differentiation of OLs [9, 52, 55]. In chronic diffuse WMI, maturation of late pre-OLs is believed to arrest at this pre-myelinating stage, contributing to myelination failure in WMI [55]. Precise mechanisms of maturational arrest are not known but likely include excessive accumulation of hyaluronic acid produced by reactive astrocytes, chronic microglial activation and changes in the cell cycle of oligodendrocyte progenitors [56]. 


\section{Grey matter injury - grey matters}

An increasing body of evidence shows that cerebral white matter injury is accompanied by disturbances of grey matter structures including cerebral and cerebellar cortex, thalamus, hippocampus and basal ganglia [57]. As in the white matter, grey matter abnormalities are also subscribed to alterations in development including altered neuronal dendritic arborisation, rather than cell death alone [32, 58]. Although data from multiple animal models have demonstrated that this predominant type of WMI is not necessarily associated with axonal damage [59, 60], it has also been shown that oligodendrocytes and neuronal functions are inherently intertwined. Oligodendrocytes play an integral role in axonal development and function, postulating that mild diffuse loss of white matter reduces the functional integrity of neuronal axons, thereby contributing to impaired neuronal growth, development and function after preterm birth [32]. Mainly two specific types of neurons, the GABAergic interneurons and subplate neurons may be of particular interest in the pathophysiology of EoP. Subplate neurons comprise a transient neuronal cell population, which is located just below cortical layer 6, and subplate neurons are essential for the development of thalamo-cortical connections and accurate formation of the distinct cortical layers [58]. The GABAergic interneurons migrate in parallel through the cerebral white matter, building connections between two other neurons and modulate neural circuitry. Both processes coincide with the peak window for vulnerability to preterm WMI and are indispensable in the development and function of cortical networks [61]. Despite that these neurons are surprisingly resistant to cell death, both show marked immature dendritic arborization and functional dysmaturation following transient hypoxemia in a fetal ovine model [61, 62] underlining their potential clinical relevance in the pathophysiology of EoP.

\section{Cerebellar injury - the underestimated impact of the little brain}

Of interest is the increasing recognition of cerebellar involvement in adverse neurodevelopmental outcomes following preterm birth. The cerebellum was previously believed to be solely responsible for motor functions and altered cerebellar function was linked to ataxia. Nowadays there is accumulating evidence showing that the cerebellum also plays an important role in the high prevalence of non-motor deficits (i.e. cognition, learning and behavior) in survivors of prematurity [63]. In particular, a correlation between autism and cerebellar injury has been established [63-65]. This is not surprising 
considering that the cerebellum contains around $80 \%$ of all neurons in the brain. Especially during the third trimester of pregnancy, the cerebellum exhibits a rapid increase in growth and development [66]. This accelerated growth is characterized by proliferation and migration of granule precursor cells from the external granular layer (EGL) to the internal granular layer (IGL) of the cerebellar cortex. These processes are essential for the structural and functional integrity of the cerebellum [66]. At this stage of development, the cerebellum is particularly vulnerable to insults that disturb normal development (e.g. hypoxia-ischemia, inflammation). Experimental studies in fetal sheep [67] have shown that perinatal asphyxia resulted in increased neuronal death, reduction of cerebellar strata, increased oxidative stress and decreased astrocytes in the preterm cerebellum. These recent findings indicate that cerebellar injury contributes to the morbidities associated with 'encephalopathy of prematurity' and highlight the importance of protection of the cerebellum to prevent long-term neurodevelopmental impairments.

\section{Diagnostics}

In contrast to the previously common cystic white matter lesions that were easily detectable by ultrasound and MRI, the new diffuse WMI with microscopic, punctate lesions and altered white matter development is extremely difficult to detect by ultrasound and conventional MRI (T1, T2) [68]. This diffuse type of WMI that is most frequently observed in preterm infants is only in $20 \%$ of all cases detected by conventional early MRI. In the recent years, brain-imaging techniques, such as Diffusion Tensor Imaging (DTI) and functional magnetic resonance imaging (fMRI) have allowed scientists to study the brain in more structural detail and determine how groups of neurons function [4]. To optimize sensitivity and specificity of these new imaging modalities correlating histological pathological changes in the fetal brain with imaging markers is essential. Considering that all potential neuroprotective treatments have a limited window of opportunity, earlier diagnosis with the help of MRI, especially DWI, may help to develop and optimize new treatment strategies and prognostic markers for more accurate prediction of outcome. 


\section{New neuroprotective strategies - enhancing endogenous regeneration}

In an ideal world, we would first like to prevent preterm birth. Over the last years, interventions including adjusted protocols for assisted reproductive technologies, progesterone supplementation, smoking cessation and reduction of non-medically indicated labor induction or caesarean delivery resulted in a relative reduction of only $5 \%$ of preterm birth [69]. Timely diagnosis and treatment of intra-amniotic infections as the main underlying mechanisms of preterm birth using antibiotic treatment and fetal delivery has shown to improve maternal and neonatal outcomes [70]. Moreover, antibiotics for PPROM are effective in reducing the risk of early morbidities including respiratory distress syndrome and neonatal infections but without a significant improvement of mortality rates [71]. The current considerations of preferential antibiotic treatment in chorioamnionitis mostly do not cover Mycoplasma species though they are the most common isolates in chorioamnionitis [72]. Unfortunately, randomized controlled trials regarding the effectiveness and superiority of antibiotic treatment to treat chorioamnionitis are limited [73]. A new clinical trial to prevent chorioamnionitis and subsequent preterm birth by antibiotic treatment of pregnant women with a positive microbiological profile at 18 weeks of gestation is currently ongoing (clinicaltrials.gov). Nevertheless, antibiotic treatment only prevents adverse outcomes related to bacterial infections, whereas viral and fungal species are not eradicated by antibiotic treatments. In two recent case reports of intra-amniotic $C$. albicans infections intraamniotic injections of Fluconazole, supplemented with oral and vaginal treatment, resulted in prolonged pregnancy and survival of both neonates [17]. Therefore, in chapter 4 we studied the effects of intra-amniotic Flucanozole administration on the development of the preterm brain following intrauterine $C$. albicans exposure.

Alternatively, besides preventing preterm birth and eradication of the microbe responsible for the intrauterine infection, we need to aim at 
preventing the inflammation- and/or HI-induced adverse effects on brain development. As already outlined in paragraph 1.2, the pathophysiology of EoP includes multiple factors contributing to alterations in healthy brain development or injury ultimately leading to impaired brain functions. As a response to this, endogenous defensive mechanisms will be activated; though when the high demand for regeneration cannot be met this will eventually result in a self-perpetuating cycle of injury. Therefore, we propose that on the search for new neuroprotective treatment strategies to restore a healthy balance within the brain, one should focus on enhancing the endogenous repair mechanisms and stimulation of regeneration.

Since the neuroinflammatory response, characterized by the disproportionate activation of microglia, is a key mediator in the induction of brain injury, targeting these microglia seem a reasonable and promising treatment strategy to prevent adverse neurodevelopmental outcomes. Nevertheless, depleting microglia from the brain has demonstrated to increase the lesion site by $60 \%$ after (neonatal) stroke [74, 75]. Besides its noxious effects, microglia are an indispensable player in the endogenous defense mechanisms by inducing anti-inflammatory cascades and activating endogenous repair mechanisms $[36,37]$. The classical dichotomous classification of microglial functions into M1 (pro-inflammatory) and M2 (anti-inflammatory) microglia is oversimplified compared to the in vivo situation and therefore obsolete. Nowadays it is appreciated that microglia display a broad array and continuum of phenotypes in vivo that can rapidly change in a context-dependent manner and thereby attenuating or exacerbating injury [36]. These negative feedback mechanisms slow down the progression of inflammation and protect the central nervous system from the various injurious effects of neuroinflammation, thereby preventing extensive inflammation induced brain damage. In order to devise effective treatment for EoP, it is important to reach an adequate balance between reducing excessive inflammation but maintaining basal defense mechanisms (immunomodulation) and regeneration of damaged cells [76, 77].

In the last decades, extensive research has been performed to find new neuroprotective treatment options to protect the immature brain. Extensive experimental studies have shown that strong immunomodulatory and regenerative capacities are met by erythropoietin (EPO) and stem cell-based therapies $[78,79]$. 
EPO is an important cytokine for brain development [80]. Multiple experimental and clinical studies have demonstrated efficacy of EPO administration to prevent injury to the preterm brain, including severe periventricular leukomalacia, without adverse effects [81]. However, in contrast, other clinical trials do not report improvement in neurodevelopment following EPO treatment [82, 83]. Effects of EPO are mediated by its receptor, which is abundantly present on (pre)oligodendrocytes, astrocytes, microglia and neurons. EPO binding triggers phosphorylation of two monomers, which in turn phosphorylates and activates the signaling kinase Jak-2 facilitating effects including its anti-inflammatory, anti-oxidative and anti-apoptotic properties [81]. In addition, EPO enhances neuro- and oligodendrogenesis, oligodendrocyte maturation and myelin production which are indispensable events in injury repair and normal neurodevelopment. A potential explanation for the reported inconsistencies in clinical outcomes following EPO treatment might be the heterogeneity between study cohorts and/or differences in EPO signaling in response to inflammatory triggers. This latter aspect is studied in chapter 3 .

Cell based therapies are emerging as promising neuroprotective treatment for the prevention of EoP. Stem cells can indirectly amend endogenous cell responses by secretion of growth factors and cytokines which are mediating tissue repair [84]. In addition, stem cells possess the potential for self-renewal and the ability to differentiate into mature cell lineages which is beneficial for brain repair after injury. A great variety in different types of stem cells are currently under investigation including mesenchymal stem/stromal cells (MSCs) and endothelial progenitor cells (EPCs) obtained from placental tissue or umbilical cord blood. More recently, Multipotent Adult Derived Stem Cells (MAPCs) (chapter 5), a subpopulation of MSCs, have gathered much attention. Compared to MSCs, MAPCs demonstrate a number of favorable characteristics including more genetic stability, extensive expansion capacity, and lower immunogenicity profiles that support allogeneic utility and "off the shelf" availability [85]. Moreover, their smaller size enables them to pass through the pulmonary vasculature more easily. The neuroprotective effects of both MSCs and MAPCs are largely mediated through paracrine activities including the release of extracellular vesicles (MSC-EVs) containing RNAs, lipids and proteins as potential favorable mediators (chapter 6). MSCs and MSC-EVs have been shown to exert similar positive effects in various preclinical disease models. In addition, MSC-EVs are not metabolically active which avoids potential 
concerns raised by administration of living cells including possible tumor formation.

Considering the important role of a disrupted BBB in the pathophysiological mechanism of EoP, restoring this integrity by enforcing endothelial cell connections could be an additional target for new neuroprotective treatments. AnnexinA1 (ANXA1) has recently been identified as an essential endogenous regulator of blood-brain barrier integrity in neurodegenerative diseases and administration of exogenous ANXA1 ameliorates BBB permeability [86, 87]. Classically, ANXA1 acts as important downstream molecule of glucocorticoids via formyl peptide receptors (FPR) and is involved in resolution of inflammation by decreasing leukocyte extravasation in the periphery [88]. However, the role of ANXA1 in the brain is not fully understood. Enhanced expression of ANXA1 has been reported in lesions of the brain in Parkinson's disease and multiple sclerosis. During brain development ANXA1 is already present in microglia and in brain microvascular endothelial cells where it is suggested to regulate BBB integrity. In endothelial cells lining the BBB, ANXA1 binds to $\beta$-actin hereby promoting cytoskeleton formation and facilitating establishment of tight junctions [87]. In addition, ANXA1 can act on the membrane formyl peptide receptor 2 (FPR2) to inhibit the activity of ras homolog gene family, member A (RhoA) which promotes cytoskeletal stability and enhances tight junction formation, hence contributing to BBB integrity.

\section{Translational animal model}

In the studies reported in this thesis we used a preclinical model of pregnant sheep to study the effects of in utero inflammation or global HI on the development of the fetal brain. The developmental biology of the ovine fetus closely resembles the human situation [89]. Essential key developmental processes including oligodendrocyte maturation and myelination of the brain follow the same time course in sheep as in humans. In particular, myelination of the ovine and human brain already starts in utero, as compared to rodents where this only starts postnatally. Moreover, the human and sheep brain are gyrencephalic, whereas rodents are lisenchephalic. Despite these differences, rodent models have been 
very helpful in obtaining more mechanistic insight in pathophysiological processes underlying EoP. Yet, the key features of EoP that are critical to model in animal paradigms including a cerebral inflammatory response and diffuse white matter injury have been previously observed in fetal sheep exposed to in utero inflammation or HI [90]. Interplay between human and animal analysis in translational research is valuable since the two types of approaches can inform, build upon and supplement each other towards the complete elucidation of EoP and its treatment. However, concerns about rodent models regarding differences in responses to pharmacological agents compared to humans might hamper translation to the clinical situation [91]. Therefore, we used the fetal ovine model to test potential new neuroprotective treatments to expedite clinical application. For the in utero inflammation model we used in this thesis bacteria (Ureaplasma parvum), bacterial products (E. Coli-derived Lipopolysaccharide) or yeast species (Candida albicans) were administered directly in the amniotic fluid of pregnant sheep at different gestational ages and with different exposure periods (inflammation model). A global hypoxic-ischemic perinatal insult was mimicked by transient umbilical cord occlusion (UCO) followed by a reperfusion period before preterm delivery by a caesarean section (HI model).

\section{Scope of this thesis}

In this thesis, different insults and treatments are tested separately. The strictly defined circumstances in a preclinical model allow the concise testing of an intervention and in parallel avoid interferences by controlling additional parameters which potentially influence outcome measures. To study the effects of chorioamnionitis we used a model of intra-uterine inflammation using different inflammatory triggers including E. coliderived lipopolysaccharide (LPS), Ureaplasma parvum and C. albicans. Detailed investigations of the interactions between different infectious triggers and the timing and duration of inflammatory exposures in the context of a polymicrobial syndrome such as chorioamnionitis is essential to understanding the complex and diverse neurodevelopmental outcomes after birth. 
Therefore, in chapter 2, I tested the effects of chronic intra-amniotic UP exposure in the presence or absence of a second (acute) inflammatory stimulus (Escherichia coli-derived LPS) on fetal neurodevelopment. Cerebral outcome was studied by analyzing inflammation, structural injury, epigenetic markers and lipid profile composition of the fetal brain.

Understanding the temporal effects of antenatal infection in relation to the onset of neurological injury is crucial for the development of neurotherapeutics for preterm infants. Therefore, in chapter 3, I performed a detailed analysis of the temporal dynamics of intra-amniotic LPS-induced systemic and cerebral inflammation and subsequent fetal brain injury. In addition, to optimize EPO treatment in the clinical setting we analyzed the temporal expression of the phosphorylated EPO receptor (pEPOR) in the course of this intra-amniotic inflammation.

In chapter 4, I studied the effects of a chorioamnionitis induced by Candida albicans on fetal brain development. Moreover, the therapeutic potential of a single dose of intra-amniotic Flucanozole administration on the development of the preterm brain was tested.

In chapter 5 and 6 I used a translational large animal model of global hypoxia-ischemia by umbilical cord occlusion representing the clinical condition of neonatal hy poxic-ischemic encephalopathy (HIE). In this model I studied the effects of global HI and cell based treatments on the cerebellum (chapter 5) and the blood-brain barrier (chapter 6), two regions that are both increasingly recognized in the pathophysiology of HIE. In chapter 5, I tested the effects of multipotent adult progenitor cells (MAPC) on the structure of the fetal cerebellum after global $\mathrm{HI}$ by immunohistochemistry of inflammatory, white matter and neuronal changes. Moreover, I studied if the histological changes that I have found could also be detected by diffusion weighted MRI.

In chapter 6 I tested whether stem cell derived extracellular vesicles (containing ANXA1) protect the integrity of the blood-brain barrier after global HI. Besides the use of the large sheep model, I studied more mechanistic insights in a widely accepted BBB in vitro model in which transendothelial electrical resistance (TEER) was measured on fetal primary ECs as a marker for endothelial integrity. 


\section{References}

1. Stiles, J., and T. L. Jernigan. “The Basics of Brain Development." Neuropsychol Rev 20, no. 4 (2010): 327-48.

2. Kinney, H. C., and J. J. Volpe. "Modeling the Encephalopathy of Prematurity in Animals: The Important Role of Translational Research." Neurol Res Int 2012 (2012): 295389.

3. Moreira, R. S., L. C. Magalhaes, and C. R. Alves. "Effect of Preterm Birth on Motor Development, Behavior, and School Performance of School-Age Children: A Systematic Review." J Pediatr (Rio J) 90, no. 2 (2014): 119-34.

4. Ment, L. R., and B. R. Vohr. "Preterm Birth and the Developing Brain." Lancet Neurol 7, no. 5 (2008): 378-9.

5. Blencowe, H., S. Cousens, M. Z. Oestergaard, D. Chou, A. B. Moller, R. Narwal, A. Adler, C. Vera Garcia, S. Rohde, L. Say, and J. E. Lawn. “National, Regional, and Worldwide Estimates of Preterm Birth Rates in the Year 2010 with Time Trends since 1990 for Selected Countries: A Systematic Analysis and Implications." Lancet 379, no. 9832 (2012): 2162-72.

6. Lawn, J. E., R. Davidge, V. K. Paul, S. von Xylander, J. de Graft Johnson, A. Costello, M. V. Kinney, J. Segre, and L. Molyneux. "Born Too Soon: Care for the Preterm Baby." Reprod Health 10 Suppl 1 (2013): S5.

7. Liu, L., S. Oza, D. Hogan, Y. Chu, J. Perin, J. Zhu, J. E. Lawn, S. Cousens, C. Mathers, and R. E. Black. "Global, Regional, and National Causes of under-5 Mortality in 2000-15: An Updated Systematic Analysis with Implications for the Sustainable Development Goals." Lancet 388, no. 10063 (2016): 3027-35.

8. Blencowe, H., S. Cousens, D. Chou, M. Oestergaard, L. Say, A. B. Moller, M. Kinney, J. Lawn, and Group Born Too Soon Preterm Birth Action. "Born Too Soon: The Global Epidemiology of 15 Million Preterm Births." Reprod Health 10 Suppl 1 (2013): S2.

9. Volpe, J. J. "The Encephalopathy of Prematurity--Brain Injury and Impaired Brain Development Inextricably Intertwined." Semin Pediatr Neurol 16, no. 4 (2009): 167-78.

10. Back, S. A., and S. P. Miller. "Brain Injury in Premature Neonates: A Primary Cerebral Dysmaturation Disorder?" Ann Neurol 75, no. 4 (2014): 469-86.

11. Hagberg, H., C. Mallard, D. M. Ferriero, S. J. Vannucci, S. W. Levison, Z. S. Vexler, and P. Gressens. "The Role of Inflammation in Perinatal Brain Injury." Nat Rev Neurol 11, no. 4 (2015): 192-208.

12. Penn, A. A., P. Gressens, B. Fleiss, S. A. Back, and V. Gallo. “Controversies in Preterm Brain Injury." Neurobiol Dis 92, no. Pt A (2016): 90-101. 
13. Galinsky, R., G. R. Polglase, S. B. Hooper, M. J. Black, and T. J. Moss. "The Consequences of Chorioamnionitis: Preterm Birth and Effects on Development." J Pregnancy 2013 (2013): 412831.

14. Kim, C. J., R. Romero, P. Chaemsaithong, N. Chaiyasit, B. H. Yoon, and Y. M. Kim. "Acute Chorioamnionitis and Funisitis: Definition, Pathologic Features, and Clinical Significance." Am J Obstet Gynecol 213, no. 4 Suppl (2015): S29-52.

15. Goldenberg, R. L., J. C. Hauth, and W. W. Andrews. "Intrauterine Infection and Preterm Delivery." N Engl J Med 342, no. 20 (2000): 1500-7.

16. Maki, Y., M. Fujisaki, Y. Sato, and H. Sameshima. "Candida Chorioamnionitis Leads to Preterm Birth and Adverse Fetal-Neonatal Outcome." Infect Dis Obstet Gynecol 2017 (2017): 9060138.

17. Bean, L. M., J. R. Jackson, W. J. Dobak, T. R. Beiswenger, and J. A. Thorp. “Intra-Amniotic Fluconazole Therapy for Candida Albicans Intra-Amniotic Infection." Obstet Gynecol 121, no. 2 Pt 2 Suppl 1 (2013): 452-4.

18. Tsai, N. Y., S. S. Laforce-Nesbitt, R. Tucker, and J. M. Bliss. "A Murine Model for Disseminated Candidiasis in Neonates." Pediatr Res 69, no. 3 (2011): 189-93.

19. Romero, R., J. Espinoza, L. F. Goncalves, J. P. Kusanovic, L. A. Friel, and J. K. Nien. "Inflammation in Preterm and Term Labour and Delivery." Semin Fetal Neonatal Med 11, no. 5 (2006): 317-26.

20. Elovitz, M. A., A. G. Brown, K. Breen, L. Anton, M. Maubert, and I. Burd. "Intrauterine Inflammation, Insufficient to Induce Parturition, Still Evokes Fetal and Neonatal Brain Injury." Int J Dev Neurosci 29, no. 6 (2011): 663-71.

21. Arayici, S., G. Kadioglu Simsek, M. Y. Oncel, Z. Eras, F. E. Canpolat, S. S. Oguz, N. Uras, S. Zergeroglu, and U. Dilmen. "The Effect of Histological Chorioamnionitis on the ShortTerm Outcome of Preterm Infants $</=32$ Weeks: A Single-Center Study." J Matern Fetal Neonatal Med 27, no. 11 (2014): 1129-33.

22. Lu, H. Y., Q. Zhang, Q. X. Wang, and J. Y. Lu. “Contribution of Histologic Chorioamnionitis and Fetal Inflammatory Response Syndrome to Increased Risk of Brain Injury in Infants with Preterm Premature Rupture of Membranes." Pediatr Neurol 61 (2016): 94-98 e1.

23. Korzeniewski, S. J., R. Romero, J. Cortez, A. Pappas, A. G. Schwartz, C. J. Kim, J. S. Kim, Y. M. Kim, B. H. Yoon, T. Chaiworapongsa, and S. S. Hassan. "A “Multi-Hit" Model of Neonatal White Matter Injury: Cumulative Contributions of Chronic Placental Inflammation, Acute Fetal Inflammation and Postnatal Inflammatory Events." J Perinat Med 42, no. 6 (2014): 731-43.

24. Soraisham, A. S., C. Trevenen, S. Wood, N. Singhal, and R. Sauve. "Histological Chorioamnionitis and Neurodevelopmental Outcome in Preterm Infants." J Perinatol 33, no. 1 (2013): 70-5.

25. Gotsch, F., R. Romero, J. P. Kusanovic, S. Mazaki-Tovi, B. L. Pineles, O. Erez, J. Espinoza, and S. S. Hassan. “The Fetal Inflammatory Response Syndrome.” Clin Obstet Gynecol 50, no. 3 (2007): 652-83. 
26. Gomez, R., R. Romero, F. Ghezzi, B. H. Yoon, M. Mazor, and S. M. Berry. "The Fetal Inflammatory Response Syndrome.” Am J Obstet Gynecol 179, no. 1 (1998): 194-202.

27. Yoon, B. H., J. K. Jun, R. Romero, K. H. Park, R. Gomez, J. H. Choi, and I. O. Kim. “Amniotic Fluid Inflammatory Cytokines (Interleukin-6, Interleukin-1beta, and Tumor Necrosis Factor-Alpha), Neonatal Brain White Matter Lesions, and Cerebral Palsy." Am J Obstet Gynecol 177, no. 1 (1997): 19-26.

28. Pacora, P., T. Chaiworapongsa, E. Maymon, Y. M. Kim, R. Gomez, B. H. Yoon, F. Ghezzi, S. M. Berry, F. Qureshi, S. M. Jacques, J. C. Kim, N. Kadar, and R. Romero. “Funisitis and Chorionic Vasculitis: The Histological Counterpart of the Fetal Inflammatory Response Syndrome." J Matern Fetal Neonatal Med 11, no. 1 (2002): 18-25.

29. Pappas, A., D. E.Kendrick, S. Shankaran, B.J.Stoll, E.F. Bell, A.R.Laptook, M.C. Walsh, A. Das, E. C. Hale, N. S. Newman, R. D. Higgins, Health Eunice Kennedy Shriver National Institute of Child, and Network Human Development Neonatal Research. "Chorioamnionitis and Early Childhood Outcomes among Extremely Low-Gestational-Age Neonates." JAMA Pediatr 168, no. 2 (2014): 137-47.

30. Malaeb, S., and O. Dammann. "Fetal Inflammatory Response and Brain Injury in the Preterm Newborn." J Child Neurol 24, no. 9 (2009): 1119-26.

31. Douglas-Escobar, M., and M. D. Weiss. "Hypoxic-Ischemic Encephalopathy: A Review for the Clinician." JAMA Pediatr 169, no. 4 (2015): 397-403.

32. Galinsky, R., C. A. Lear, J. M. Dean, G. Wassink, S. K. Dhillon, M. Fraser, J. O. Davidson, L. Bennet, and A. J. Gunn. "Complex Interactions between Hypoxia-Ischemia and Inflammation in Preterm Brain Injury." Dev Med Child Neurol 60, no. 2 (2018): 126-33.

33. Wang, Q., H. Lv, L. Lu, P. Ren, and L. Li. "Neonatal Hypoxic-Ischemic Encephalopathy: Emerging Therapeutic Strategies Based on Pathophysiologic Phases of the Injury." J Matern Fetal Neonatal Med (2018): 1-8.

34. Sato, K. "Effects of Microglia on Neurogenesis." Glia 63, no. 8 (2015): 1394-405.

35. Lodygensky, G. A., N. Kunz, E. Perroud, E. Somm, V. Mlynarik, P. S. Huppi, R. Gruetter, and S. V. Sizonenko. "Definition and Quantification of Acute Inflammatory White Matter Injury in the Immature Brain by Mri/Mrs at High Magnetic Field." Pediatr Res 75, no. 3 (2014): 415-23.

36. Mallard, C., M. E. Tremblay, and Z. S. Vexler. "Microglia and Neonatal Brain Injury." Neuroscience 405 (2019): 68-76.

37. Pierre, W. C., P. L. P. Smith, I. Londono, S. Chemtob, C. Mallard, and G. A. Lodygensky. "Neonatal Microglia: The Cornerstone of Brain Fate." Brain Behav Immun 59 (2017): 333-45.

38. Fernandez de Cossio, L., A. Guzman, S. van der Veldt, and G. N. Luheshi. "Prenatal Infection Leads to Asd-Like Behavior and Altered Synaptic Pruning in the Mouse Offspring." Brain Behav Immun 63 (2017): 88-98.

39. Nitsos, I., S. M. Rees, J. Duncan, B. W. Kramer, R. Harding, J. P. Newnham, and T. J. Moss. "Chronic Exposure to Intra-Amniotic Lipopolysaccharide Affects the Ovine Fetal Brain." J Soc Gynecol Investig 13, no. 4 (2006): 239-47. 
40. Dean, J. M., Y. van de Looij, S. V. Sizonenko, G. A. Lodygensky, F. Lazeyras, H. Bolouri, I. Kjellmer, P. S. Huppi, H. Hagberg, and C. Mallard. "Delayed Cortical Impairment Following Lipopolysaccharide Exposure in Preterm Fetal Sheep." Ann Neurol 70, no. 5 (2011): 846-56.

41. Favrais, G., Y. van de Looij, B. Fleiss, N. Ramanantsoa, P. Bonnin, G. Stoltenburg-Didinger, A. Lacaud, E. Saliba, O. Dammann, J. Gallego, S. Sizonenko, H. Hagberg, V. Lelievre, and P. Gressens. "Systemic Inflammation Disrupts the Developmental Program of White Matter." Ann Neurol 70, no. 4 (2011): 550-65.

42. Cowell, R. M., J. M. Plane, and F. S. Silverstein. "Complement Activation Contributes to Hypoxic-Ischemic Brain Injury in Neonatal Rats." J Neurosci 23, no. 28 (2003): 9459-68.

43. Mottahedin, A., M. Ardalan, T. Chumak, I. Riebe, J. Ek, and C. Mallard. "Effect of Neuroinflammation on Synaptic Organization and Function in the Developing Brain: Implications for Neurodevelopmental and Neurodegenerative Disorders." Front Cell Neurosci 11 (2017): 190.

44. Moretti, R., J. Pansiot, D. Bettati, N. Strazielle, J. F. Ghersi-Egea, G. Damante, B. Fleiss, L. Titomanlio, and P. Gressens. "Blood-Brain Barrier Dysfunction in Disorders of the Developing Brain." Front Neurosci 9 (2015): 40.

45. Lee, W. L. A., A. T. Michael-Titus, and D. K. Shah. "Hypoxic-Ischaemic Encephalopathy and the Blood-Brain Barrier in Neonates." Dev Neurosci 39, no. 1-4 (2017): 49-58.

46. Carson, M. J., J. C. Thrash, and B. Walter. "The Cellular Response in Neuroinflammation: The Role of Leukocytes, Microglia and Astrocytes in Neuronal Death and Survival." Clin Neurosci Res 6, no. 5 (2006): 237-45.

47. Burd, I., A. Brown, J. M. Gonzalez, J. Chai, and M. A. Elovitz. "A Mouse Model of Term Chorioamnionitis: Unraveling Causes of Adverse Neurological Outcomes." Reprod Sci 18, no. 9 (2011): 900-7.

48. Yang, Y., H. Higashimori, and L. Morel. "Developmental Maturation of Astrocytes and Pathogenesis of Neurodevelopmental Disorders." J Neurodev Disord 5, no. 1 (2013): 22.

49. Rutherford, M. A., V. Supramaniam, A. Ederies, A. Chew, L. Bassi, M. Groppo, M. Anjari, S. Counsell, and L. A. Ramenghi. "Magnetic Resonance Imaging of White Matter Diseases of Prematurity." Neuroradiology 52, no. 6 (2010): 505-21.

50. Alexandrou, G., G. Martensson, B. Skiold, M. Blennow, U. Aden, and B. Vollmer. “White Matter Microstructure Is Influenced by Extremely Preterm Birth and Neonatal Respiratory Factors." Acta Paediatr 103, no. 1 (2014): 48-56.

51. van Tilborg, E., C. G. M. de Theije, M. van Hal, N. Wagenaar, L. S. de Vries, M. J. Benders, D. H. Rowitch, and C. H. Nijboer. "Origin and Dynamics of Oligodendrocytes in the Developing Brain: Implications for Perinatal White Matter Injury." Glia 66, no. 2 (2018): 221-38.

52. Volpe, J. J., H. C. Kinney, F. E. Jensen, and P. A. Rosenberg. "The Developing Oligodendrocyte: Key Cellular Target in Brain Injury in the Premature Infant." Int J Dev Neurosci 29, no. 4 (2011): 423-40. 
53. Back, S. A., B. H. Han, N. L. Luo, C. A. Chricton, S. Xanthoudakis, J. Tam, K. L. Arvin, and D. M. Holtzman. "Selective Vulnerability of Late Oligodendrocyte Progenitors to HypoxiaIschemia." J Neurosci 22, no. 2 (2002): 455-63.

54. Back, S. A., X. Gan, Y. Li, P. A. Rosenberg, and J. J. Volpe. "Maturation-Dependent Vulnerability of Oligodendrocytes to Oxidative Stress-Induced Death Caused by Glutathione Depletion." J Neurosci 18, no. 16 (1998): 6241-53.

55. Segovia, K. N., M. McClure, M. Moravec, N. L. Luo, Y. Wan, X. Gong, A. Riddle, A. Craig, J. Struve, L. S. Sherman, and S. A. Back. "Arrested Oligodendrocyte Lineage Maturation in Chronic Perinatal White Matter Injury." Ann Neurol 63, no. 4 (2008): 520-30.

56. Back, S. A., and P. A. Rosenberg. "Pathophysiology of Glia in Perinatal White Matter Injury." Glia 62, no. 11 (2014): 1790-815.

57. Keunen, K., K. J. Kersbergen, F. Groenendaal, I. Isgum, L. S. de Vries, and M. J. Benders. "Brain Tissue Volumes in Preterm Infants: Prematurity, Perinatal Risk Factors and Neurodevelopmental Outcome: A Systematic Review." J Matern Fetal Neonatal Med 25 Suppl 1 (2012): 89-100.

58. Volpe, J. J. "Dysmaturation of Premature Brain: Importance, Cellular Mechanisms, and Potential Interventions." Pediatr Neurol (2019).

59. Riddle, A., J. Maire, X. Gong, K. X. Chen, C. D. Kroenke, A. R. Hohimer, and S. A. Back. "Differential Susceptibility to Axonopathy in Necrotic and Non-Necrotic Perinatal White Matter Injury." Stroke 43, no. 1 (2012): 178-84.

60. van Tilborg, E., E. J. M. Achterberg, C. M. van Kammen, A. van der Toorn, F. Groenendaal, R. M. Dijkhuizen, C. J. Heijnen, Ljmj Vanderschuren, Mnjl Benders, and C. H. A. Nijboer. “Combined Fetal Inflammation and Postnatal Hypoxia Causes Myelin Deficits and AutismLike Behavior in a Rat Model of Diffuse White Matter Injury." Glia 66, no. 1 (2018): 78-93.

61. McClendon, E., K. Chen, X. Gong, E. Sharifnia, M. Hagen, V. Cai, D. C. Shaver, A. Riddle, J. M. Dean, A. J. Gunn, C. Mohr, J. S. Kaplan, D. J. Rossi, C. D. Kroenke, A. R. Hohimer, and S. A. Back. "Prenatal Cerebral Ischemia Triggers Dysmaturation of Caudate Projection Neurons." Ann Neurol 75, no. 4 (2014): 508-24.

62. McClendon, E., D. C. Shaver, K. Degener-O’Brien, X. Gong, T. Nguyen, A. HoerderSuabedissen, Z. Molnar, C. Mohr, B. D. Richardson, D. J. Rossi, and S. A. Back. "Transient Hypoxemia Chronically Disrupts Maturation of Preterm Fetal Ovine Subplate Neuron Arborization and Activity." J Neurosci 37, no. 49 (2017): 11912-29.

63. Stoodley, C. J., and C. Limperopoulos. "Structure-Function Relationships in the Developing Cerebellum: Evidence from Early-Life Cerebellar Injury and Neurodevelopmental Disorders." Semin Fetal Neonatal Med 21, no. 5 (2016): 356-64.

64. Limperopoulos, C., H. Bassan, K. Gauvreau, R. L. Robertson, Jr., N. R. Sullivan, C. B. Benson, L. Avery, J. Stewart, J. S. Soul, S. A. Ringer, J. J. Volpe, and A. J. duPlessis. “Does Cerebellar Injury in Premature Infants Contribute to the High Prevalence of Long-Term Cognitive, Learning, and Behavioral Disability in Survivors?" Pediatrics 120, no. 3 (2007): 584-93.

65. Tsai, P. T., C. Hull, Y. Chu, E. Greene-Colozzi, A. R. Sadowski, J. M. Leech, J. Steinberg, J. 
N. Crawley, W. G. Regehr, and M. Sahin. "Autistic-Like Behaviour and Cerebellar Dysfunction in Purkinje Cell Tsc1 Mutant Mice." Nature 488, no. 7413 (2012): 647-51.

66. Volpe, J. J. "Cerebellum of the Premature Infant: Rapidly Developing, Vulnerable, Clinically Important." J Child Neurol 24, no. 9 (2009): 1085-104.

67. Hutton, L. C., E. Yan, T. Yawno, M. Castillo-Melendez, J. J. Hirst, and D. W. Walker. “Injury of the Developing Cerebellum: A Brief Review of the Effects of Endotoxin and Asphyxial Challenges in the Late Gestation Sheep Fetus." Cerebellum 13, no. 6 (2014): 777-86.

68. Jin, C., I. Londono, C. Mallard, and G. A. Lodygensky. "New Means to Assess Neonatal Inflammatory Brain Injury." J Neuroinflammation 12 (2015): 180.

69. Chang, H. H., J. Larson, H. Blencowe, C. Y. Spong, C. P. Howson, S. Cairns-Smith, E. M. Lackritz, S. K. Lee, E. Mason, A. C. Serazin, S. Walani, J. L. Simpson, J. E. Lawn, and group Born Too Soon preterm prevention analysis. "Preventing Preterm Births: Analysis of Trends and Potential Reductions with Interventions in 39 Countries with Very High Human Development Index." Lancet 381, no. 9862 (2013): 223-34.

70. Johnson, C. T., R. R. Adami, and A. Farzin. "Antibiotic Therapy for Chorioamnionitis to Reduce the Global Burden of Associated Disease." Front Pharmacol 8 (2017): 97.

71. Cousens, S., H. Blencowe, M. Gravett, and J. E. Lawn. “Antibiotics for Pre-Term Pre-Labour Rupture of Membranes: Prevention of Neonatal Deaths Due to Complications of Pre-Term Birth and Infection." Int J Epidemiol 39 Suppl 1 (2010): i134-43.

72. Tita, A. T., and W. W. Andrews. "Diagnosis and Management of Clinical Chorioamnionitis." Clin Perinatol 37, no. 2 (2010): 339-54.

73. Chapman, E., L. Reveiz, E. Illanes, and X. Bonfill Cosp. “Antibiotic Regimens for Management of Intra-Amniotic Infection.” Cochrane Database Syst Rev, no. 12 (2014): CD010976.

74. Szalay, G., B. Martinecz, N. Lenart, Z. Kornyei, B. Orsolits, L. Judak, E. Csaszar, R. Fekete, B. L. West, G. Katona, B. Rozsa, and A. Denes. "Microglia Protect against Brain Injury and Their Selective Elimination Dysregulates Neuronal Network Activity after Stroke." Nat Commun 7 (2016): 11499.

75. Faustino, J. V., X. Wang, C. E. Johnson, A. Klibanov, N. Derugin, M. F. Wendland, and Z. S. Vexler. "Microglial Cells Contribute to Endogenous Brain Defenses after Acute Neonatal Focal Stroke." J Neurosci 31, no. 36 (2011): 12992-3001.

76. Fleiss, B., and P. Gressens. "Tertiary Mechanisms of Brain Damage: A New Hope for Treatment of Cerebral Palsy?" Lancet Neurol 11, no. 6 (2012): 556-66.

77. van Tilborg, E., C. J. Heijnen, M. J. Benders, F. van Bel, B. Fleiss, P. Gressens, and C. H. Nijboer. "Impaired Oligodendrocyte Maturation in Preterm Infants: Potential Therapeutic Targets." Prog Neurobiol 136 (2016): 28-49.

78. Hassell, K. J., M. Ezzati, D. Alonso-Alconada, D. J. Hausenloy, and N. J. Robertson. "New Horizons for Newborn Brain Protection: Enhancing Endogenous Neuroprotection." Arch Dis Child Fetal Neonatal Ed 100, no. 6 (2015): F541-52.

79. van Bel, F., J. Vaes, and F. Groenendaal. "Prevention, Reduction and Repair of Brain Injury of the Preterm Infant." Front Physiol 10 (2019): 181. 
80. Yu, X., J. J. Shacka, J. B. Eells, C. Suarez-Quian, R. M. Przygodzki, B. Beleslin-Cokic, C. S. Lin, V. M. Nikodem, B. Hempstead, K. C. Flanders, F. Costantini, and C. T. Noguchi. “Erythropoietin Receptor Signalling Is Required for Normal Brain Development." Development 129, no. 2 (2002): 505-16.

81. van der Kooij, M. A., F. Groenendaal, A. Kavelaars, C. J. Heijnen, and F. van Bel. "Neuroprotective Properties and Mechanisms of Erythropoietin in in Vitro and in Vivo Experimental Models for Hypoxia/Ischemia." Brain Res Rev 59, no. 1 (2008): 22-33.

82. Korzeniewski, S. J., E. Allred, J. W. Logan, R. N. Fichorova, S. Engelke, K. C. Kuban, T. M. O'Shea, N. Paneth, M. Holm, O. Dammann, A. Leviton, and Elgan study investigators. "Elevated Endogenous Erythropoietin Concentrations Are Associated with Increased Risk of Brain Damage in Extremely Preterm Neonates." PLoS One 10, no. 3 (2015): e0115083.

83. Natalucci, G., B. Latal, B. Koller, C. Ruegger, B. Sick, L. Held, H. U. Bucher, J. C. Fauchere, and E. P. O. Neuroprotection Trial Group Swiss. "Effect of Early Prophylactic High-Dose Recombinant Human Erythropoietin in Very Preterm Infants on Neurodevelopmental Outcome at 2 Years: A Randomized Clinical Trial." JAMA 315, no. 19 (2016): 2079-85.

84. Paton, M. C. B., C. A. McDonald, B. J. Allison, M. C. Fahey, G. Jenkin, and S. L. Miller. "Perinatal Brain Injury as a Consequence of Preterm Birth and Intrauterine Inflammation: Designing Targeted Stem Cell Therapies." Front Neurosci 11 (2017): 200.

85. Busch, S. A., J. A. Hamilton, K. P. Horn, F. X. Cuascut, R. Cutrone, N. Lehman, R. J. Deans, A. E. Ting, R. W. Mays, and J. Silver. "Multipotent Adult Progenitor Cells Prevent MacrophageMediated Axonal Dieback and Promote Regrowth after Spinal Cord Injury." J Neurosci 31, no. 3 (2011): 944-53.

86. Cristante, E., S. McArthur, C. Mauro, E. Maggioli, I. A. Romero, M. Wylezinska-Arridge, P. O. Couraud, J. Lopez-Tremoleda, H. C. Christian, B. B. Weksler, A. Malaspina, and E. Solito. "Identification of an Essential Endogenous Regulator of Blood-Brain Barrier Integrity, and Its Pathological and Therapeutic Implications." Proc Natl Acad Sci U S A 110, no. 3 (2013): 832-41.

87. McArthur, S., R. A. Loiola, E. Maggioli, M. Errede, D. Virgintino, and E. Solito. "The Restorative Role of Annexin A1 at the Blood-Brain Barrier." Fluids Barriers CNS 13, no. 1 (2016): 17.

88. Perretti, M., and F. D'Acquisto. "Annexin A1 and Glucocorticoids as Effectors of the Resolution of Inflammation." Nat Rev Immunol 9, no. 1 (2009): 62-70.

89. Back, S. A., A. Riddle, and A. R. Hohimer. "Role of Instrumented Fetal Sheep Preparations in Defining the Pathogenesis of Human Periventricular White-Matter Injury." J Child Neurol 21, no. 7 (2006): 582-9.

90. Kuypers, E., D. Ophelders, R. K. Jellema, S. Kunzmann, A. W. Gavilanes, and B. W. Kramer. "White Matter Injury Following Fetal Inflammatory Response Syndrome Induced by Chorioamnionitis and Fetal Sepsis: Lessons from Experimental Ovine Models." Early Hum Dev 88, no. 12 (2012): 931-6.

91. Shanks, N., R. Greek, and J. Greek. "Are Animal Models Predictive for Humans?" Philos Ethics Humanit Med 4 (2009): 2. 
1 
Ruth Gussenhoven, Daan R.M.G.

Ophelders, Matthew W. Kemp, Matthew S. Payne, Owen B. Spiller, Michael L. Beeton, Sarah Stock, Bertha CilleroPastor, Florian P.Y. Barré, Ron M.A. Heeren, Lilian Kessels, Bas Stevens, Bart P. Rutten, Suhas G. Kallapur, Alan H. Jobe, Boris W. Kramer, Tim G.A.M. Wolfs

Developmental Neuroscience.

2017;39[6]:472-486. 


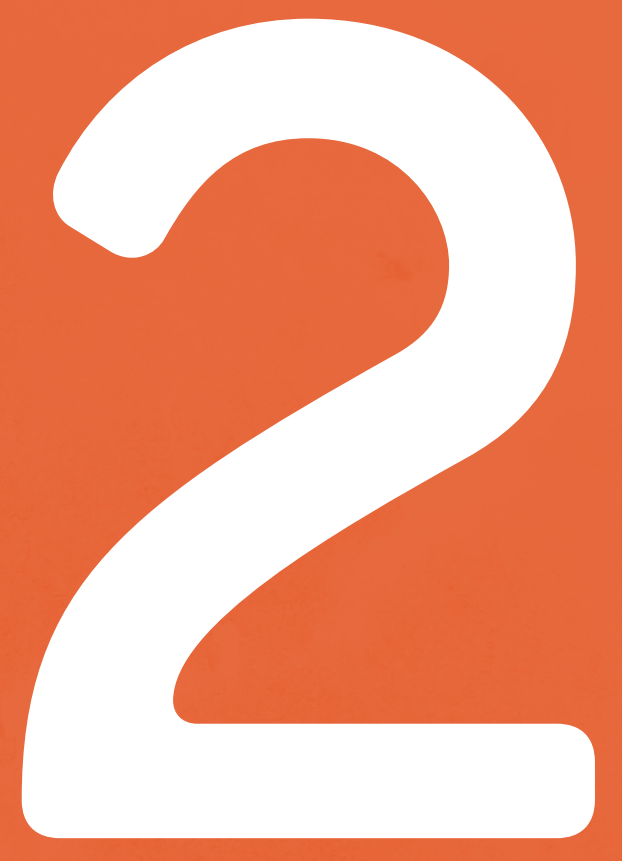

The paradoxical effects of chronic intra-amniotic Ureaplasma parvum exposure on ovine fetal brain development. 


\section{Abstract}

Chorioamnionitis is associated with adverse neurodevelopmental outcomes in preterm infants. Ureaplasma spp. are the microorganisms most frequently isolated from the amniotic fluid of women diagnosed with chorioamnionitis. However, controversy remains concerning the role of Ureaplasma spp. in the pathogenesis of neonatal brain injury. We hypothesize that re-exposure to an inflammatory trigger during the perinatal period might be responsible for the variation in brain outcome of preterms following Ureaplasma driven chorioamnionitis. To investigate these clinical scenarios, we performed a detailed multi-modal study in which ovine neurodevelopmental outcomes were assessed following chronic intra-amniotic Ureaplasma parvum (UP) infection, either alone or combined with subsequent lipopolysaccharide (LPS) exposure.

We show that chronic intra-amniotic UP exposure during the second trimester provoked a decrease of astrocytes, increased oligodendrocyte numbers and elevated 5-methylcytosine levels. In contrast, short-term LPS exposure before preterm birth induced increased microglial activation, myelin loss, elevation of 5-hydroxymethylcytosine levels and lipid profile changes. These LPS-induced changes were prevented by chronic pre-exposure to UP (preconditioning).

These data indicate that chronic UP exposure provokes dual effects on preterm brain development in utero. On one hand, prolonged UP exposure causes detrimental cerebral changes which may predispose to adverse postnatal clinical outcomes. On the other, chronic intra-amniotic UP exposure preconditions the brain against a second inflammatory hit. This study demonstrates that microbial interactions, timing and duration of inflammatory insults will determine the effects on the fetal brain. Therefore, this study helps to understand the complex and diverse postnatal neurological outcomes following UP driven chorioamnionitis. 


\section{Introduction}

Neonatal brain injury acquired during pregnancy remains a major cause of adverse neurodevelopmental outcomes throughout life [1, 2]. Chorioamnionitis which is defined as a microbial invasion and infection of the amniotic cavity is one of the most important risk factors for adverse neurodevelopmental outcomes of the newborn $[3,4]$. Ureaplasma spp. are the most common isolated micro-organisms associated with chorioamnionitis [5]. Clinical recognition of amniotic fluid infections is challenging given its asymptomatic course despite sustained fetal exposure to intrauterine inflammation, particularly during the critical period of fetal brain development [6].

Intra-amniotic exposure to Ureaplasma spp. is associated with development of fetal and neonatal brain injury [7-10]. Clinical data show that there is an increased risk for intraventricular hemorrhage and impaired neurodevelopmental outcomes later in life after intra-amniotic Ureaplasma spp. exposure $[7,9,10]$. This association was confirmed by Normann et al. who showed that intra-amniotic Ureaplasma parvum (UP) exposure resulted in increased microglial activation, delayed myelination, and disturbed cortical development of the fetal murine brain [11]. In contrast, clinical studies reported that antenatal exposure to Ureaplasma spp. and brain injury did not correlate [12, 13]. Diversities in microbial interplay, timing, duration and severity of the inflammatory response after onset of chorioamnionitis are considered to determine the neurodevelopmental outcome which most likely explains the considerable differences in antenatal UP exposure and brain injury incidences among studies [14, 6]. In particular, the onset of cerebral inflammation during the brain's most vulnerable period from 23 to 32 weeks of gestation can have detrimental consequences for the fetal brain, particularly white matter damage. Multiple animal models demonstrate that the brain becomes more (i.e. sensitization) or less (i.e. preconditioning) susceptible to a second injurious hit following pre-exposure to inflammation $[15,16]$. Besides cerebral inflammation, epigenetic mechanisms (such as DNA methylation and DNA hydroxymethylation) may mediate the 
processes leading to brain injury in response to environmental challenges in utero [17]. In line, DNA-methylation levels in genes involved in growth and development are found to be increased in premature infants with chorioamnionitis compared with infants without chorioamnionitis [18].

Moreover, alterations of phospholipids which are highly abundant in the brain and play important functions in cell membrane formation, as energy reservoirs and as precursors for second messengers (i.e. arachidonic acid (AA)) [19] have been implicated in multiple brain pathologies. In particular, changes in lipid metabolism, as seen in lysosomal storage diseases, can cause severe impaired brain function with lipids accumulating within the brain [20].

Detailed investigations of the interactions between different infectious triggers and the timing and duration of inflammatory exposures in the context of a polymicrobial syndrome such as chorioamnionitis is essential to understanding the complex and diverse neurodevelopmental outcomes after birth. We therefore investigated the effects of chronic intra-amniotic UP exposure in the presence or absence of a second (acute) inflammatory stimulus on fetal neurodevelopment. We used a well-established translational ovine model of intrauterine inflammation in which fetuses were chronically exposed to intra-amniotic UP, followed by acute exposure to Escherichia coli-derived lipopolysaccharide (LPS). Cerebral outcome was studied by analyzing inflammation, structural injury, epigenetic markers and lipid profile composition of the fetal brain. 


\section{Methods}

\section{Animal experiments}

The animal procedures were performed with approval of the animal ethics committee of the University of Western Australia (Perth, Australia).

Thirty-seven date-mated Merino ewes were randomly assigned to study groups of 5-7 animals. Fetuses of either sex were used. Ewes received an ultrasound-guided intra-amniotic injection of culture media $(2 \mathrm{~mL})$ as control or strain HPA5 of Ureaplasma parvum (UP) serovar $3(2 \times 105$ colony changing units (CCU)) at 80 days of gestation (term $\sim 150$ days). To minimize any inflammatory effects from culture media, both UP and control injections were created from stock cultures/sterile media diluted 1:100 in sterile saline. To assess the effect of an additional inflammatory hit following long-term pre-exposure with UP, both groups received a second intra-amniotic injection of 10mg Escherichia coli-derived LPS (O55:B5; Sigma-Aldrich, St. Louis, MO) at 2 or 7 days before preterm delivery at $122 \pm 2$ days of gestation or an equivalent dose of saline (SAL; controls) (Fig. 1).

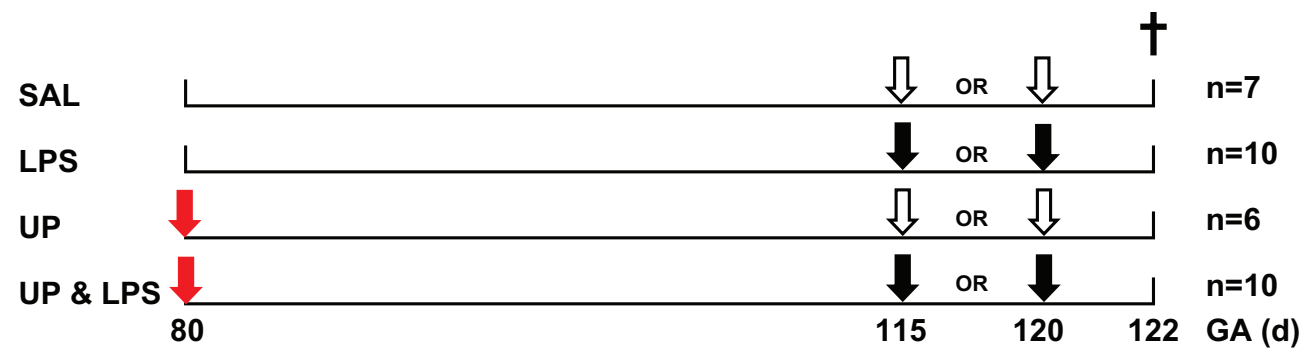

Figure 1. Experimental groups. Animals were intra-amniotically exposed to Ureaplasma parvum (UP; red arrow) for 42 days with $(n=11)$ or without $(n=6)$ subsequent lipopolysaccharide (LPS; black arrow) exposure at $2(n=5)$ or $7(n=5)$ days before preterm delivery at 122 days of gestation (GA) and sacrificed immediately after birth $(\dagger)$. Control animals received intra-amniotic injection with saline (SAL; open arrow) 


\section{Data acquisition and analysis}

All fetuses were surgically delivered via Caesarean section at $122 \pm 2$ days of gestation (equivalent of 32-34 weeks human gestation) and euthanized with intravenous pentobarbitone $(100 \mathrm{mg} / \mathrm{kg})$ immediately after birth. Amniotic fluid (AF), blood and cerebrospinal fluid (CSF) were collected at delivery and cultures for UP were performed. Brains were immersion fixed in $4 \%$ paraformaldehyde.

\section{Culture analysis of UP infection}

Samples of amniotic fluid $(1 \mathrm{ml})$ collected by amniocentesis at LPS or control saline injection, as well as plasma, CSF and amniotic fluid collected at Caesarean-section delivery were cultured for UP growth as previously described [21]. For each animal, twenty microliters of biological fluid was serially diluted 1:10 in Ureaplasma Selective Medium (Mycoplasma Experience plc., Reigate, UK) in triplicate for each sample and incubated at $37 \mathrm{oC}$. Assays were performed in 96 well plates and bacterial growth was quantified by the titration of the urease activity (conversion of urea to ammonium ions leading to $\mathrm{pH}$ color change). Plates were observed until bacteria-mediated color change ceased and the titration of the bacteria present determined.

\section{Analysis of IL-6 concentration}

The pro-inflammatory cytokine IL-6 was measured in fetal plasma as marker for systemic inflammation using a sheep-specific sandwich enzyme-linked immunosorbent assay (ELISA). Briefly, a mouse-anti-ovine monoclonal antibody (MAB1004, Millipore, Darmstadt, Germany, working concentration 1:200) was the coating antibody. Plasma samples $(100 \mu \mathrm{L})$ were loaded per well in duplicate and incubated for 2 hours at room temperature. Incubation with the detection antibody (rabbit-anti-ovine IL-6, AB1839, Millipore, Darmstadt, Germany, working concentration 1:500) was performed for 60 minutes, followed by incubation for 30 minutes with $100 \mu \mathrm{L}$ of a goatanti-rabbit-HRP (Jackson ImmunoResearch Laboratories Inc, West Grove, PA, USA, working concentration 1:500). After washing, incubation with 3,3',5,5'-tetramethylbenzidine (TMB) substrate solution for 15 minutes. The reaction was stopped by addition of $50 \mu \mathrm{L} 2 \mathrm{~N}$ sulfuric acid. The optical density (OD) was measured using a micro-plate reader at $450 \mathrm{~nm}$. Concentrations were expressed relative to a standard curve of ovine IL-6 recombinant protein (ImmunoChemistry Technologies, Bloomington, MN, USA). 


\section{Histology and immunohistochemistry}

After fixation, the right hemisphere was divided into four defined anatomical regions. The region of the posterior hippocampus/mid-thalamus was embedded in paraffin and serial coronal sections $(4 \mu \mathrm{m})$ were cut with a Leica RM2235 microtome. At this level, we analyzed the hippocampus and cerebral white matter for inflammatory and structural changes since these regions are most affected following intra-uterine infection at this developmentalstage [22]. Four slides per staining per animal were used (every 10th consecutive slide) for immunohistochemical analysis. Hematoxylin and eosin (H\&E) staining was performed for morphological and anatomical analysis. Adjacent sections were stained as previously described with a rabbit anti-ionized calcium binding adaptor molecule 1 (IBA-1) antibody (Wako Pure Chemical Industries, Osaka, Japan) for microglia, a rabbit antiglial fibrillary acidic protein (GFAP) antibody (DAKO Z0334) for astrocytes, a rat anti-myelin basic protein (MBP) antibody (Merck Millipore, MAB386) for myelin sheaths, a rabbit anti-oligodendrocyte transcription factor 2 (Olig2) antibody (Millipore, AB9610) for oligodendrocyte lineage cells, a rabbit anti-myeloperoxidase (MPO) (DAKO, A0398) for neutrophils, a mouse anti-cluster of differentiation (CD) 68 (DAKO, M0718) for active microglia/phagocytizing macrophages, a rabbit anti-CD3 (DAKO A0452) for T-lymphocytes and a mouse anti-5-Methylcytosine (5-mc) (Genway GWB-CB561B) and rabbit anti-5-Hydroxymethylcytosine (5-hmc) (Active Motif, 39769) were used as epigenetic markers.

Endogenous peroxidase activity was inactivated with $0.3 \% \mathrm{H}_{2} \mathrm{O}_{2}$ treatment (or $1 \% \mathrm{H}_{2} \mathrm{O}_{2}$ for 5-mc and 5-hmc). Antigen retrieval was performed by microwave boiling of tissue sections in citrate buffer ( $\mathrm{pH}$ 6.0). Nonspecific binding was blocked by incubation with bovine, goat or donkey serum in PBS. Sections were incubated overnight at $4^{\circ} \mathrm{C}$ with the diluted primary antibody (5-hmc 1:2500; IBA-1, GFAP and MBP 1:1000; 5-mc 1:500; Olig2, MPO 1:200). The following day sections were incubated with the specific secondary antibody and staining was enhanced with a Vectastain $A B C$ peroxidase Elite kit (Vector Laboratories Inc, Burlingame, CA) and (nickel) 3,3'-diaminobenzidine (DAB) staining. If required, appropriate background staining was performed. 


\section{Matrix assisted laser desorption ionization mass spectrometry imaging}

A more detailed molecular analysis of the cerebral tissue was done by matrix assisted laser desorption ionization mass spectrometry imaging (MALDI-MSI) to map variations in lipid profiles of the white and grey matter. MALDI-MSI to image lipid distribution can be invaluable in understanding complex lipid changes and it has been used to study these molecular patterns in models of brain injury [23]. With MALDI-MSI we avoid all extraction and purification steps for lipid analysis while retaining their spatial distribution. For this technique, post fixation tissues of controls, 42UP, 2LPS and UP\&LPS groups were frozen in liquid nitrogen and subsequently samples were cryo-sectioned ( $10 \mu \mathrm{m}$ thickness) in a cryostat (Leica CM3050S), deposited on indium tin oxide high-conductive slides (Delta Technologies, US), and stored at $-20^{\circ} \mathrm{C}$. Subsequently, the matrix solution consisting of norharman $(7 \mathrm{mg} / \mathrm{ml})$ in 2:1 chloroform:methanol was sprayed on top of the tissue section by a vibrational sprayer (Suncollect; SunChrom, Germany) for positive ion mode and 9-aminoacridine $(10 \mathrm{mg} /$ $\mathrm{mL}$ ) in $70 \%$ ethanol for negative ion mode MALDI-MSI analysis. Digital optical scans of all tissue sections were obtained prior to MALDI-MSI experiments using a 2,400 dots per inch desktop scanner. The resulting digital images were imported into the MALDI Imaging HDI software v1.4 (Waters Corporation). A MALDI-quadrupole time-of-flight SYNAPT HDMS G2Si system (Waters Corporation) operating with a 200-Hz Nd:YAG laser was configured to acquire data in positive and negative V-reflectron mode. Data were acquired at a raster size of 100 by $100 \mu \mathrm{m}$. Instrument calibration was performed using a standard calibration solution of red phosphorus.

Principal components analysis (PCA) and discriminant analysis (DA) were used to investigate spectral similarities and differences between all samples. PCA was performed as a data compression and noise filtering step before application of DA, only $1 / 4$ of the functions (216) were used as input for DA. In short, PCA is an unsupervised statistical method that aims at pooling a maximum amount of variance in a minimum number of independent variables. Data pre-treatment, PCA and DA were performed using our in-house built ChemomeTricks toolbox for MATLAB version 2014a (The MathWorks, Natick, MA, USA). The peak assignments were performed according to the bibliography and LIPID MAPS software (http://www. lipidmaps.org/). 


\section{Qualitative analysis}

H\&E-stained sections were analyzed by two independent investigators and a neuropathologist, blinded for the experimental set up, to assess overall brain structure and inflammatory changes. The sections were examined for the presence of gliosis, hemorrhages, (cytotoxic) edema and structural damage, including cyst formation.

\section{Immunohistochemical analysis}

Immunohistochemical stainings were analyzed using a light microscope (Leica DM2000) equipped with Leica QWin Pro version 3.4.0 software (Leica Microsystems, Mannheim, Germany). Regions of interest of the white matter and hippocampus were defined as previously described [24]. These regions were chosen since they are most affected by intra-uterine infections at this developmental stage. In the white matter 4-6 adjacent images were taken at 100x magnification and from the hippocampus one image at 20x magnification was taken. To assess regional vulnerability within the hippocampus separate images were taken at 200x magnification of the Cornu Amonis (CA) 1\&2, 3 and 4 and the dentate gyrus (DG). Area fractions of IBA-1, GFAP, MBP, 5-mc and 5-hmc expression were determined using a standard threshold to detect positive staining with Leica QWin Pro V 3.5.1 software (Leica, Rijswijk, the Netherlands). Regions of interest were delineated in the image with large blood vessels and artefacts excluded from analysis. Since the level of DNA methylation and hydroxymethylation can differ per cell, the integrated density of 5-mc and 5 -hmc was calculated by multiplying the area fraction by the mean gray value, and these values were normalized to the data of the control group as previously described by Lardenoije et al [25]. In addition to area fraction analysis, IBA-1 and GFAP positive cells were counted in 3 fields of view within the regions of interest at a magnification of 400x. The Olig2 positive cells were counted using Qwin software and expressed as cells $/ \mathrm{mm} 2$. MPO positive cells were counted focusing on the cerebral vasculature, meninges and choroid plexus. To quantify the density (cells per mm2) of MPO-, CD68- and CD3-positive cells, representative images of the choroid plexus present in the lateral ventricles aligning the hippocampus were counted using ImageJ software (Rasband, W.S., Image J US National Institutes of Health, Bethesda, Maryland, USA; RRID:SCR_003070). The images were acquired and analyzed by an independent observer who was blinded to the experimental groups. 


\section{Data analysis}

All values are shown as means with $95 \%$ confidence interval or standard deviations with significance level at $p<0.05$. Comparison between different experimental groups was performed with analysis of variance (ANOVA) or with a random intercept mixed model in case of repeated measurements per animal (e.g., different sections per brain). We applied log-transformation to obtain normal distributed data when data or variables were positively skewed before statistical testing. Statistical analysis was performed with IBM SPSS Statistics Version 22.0 (IBM COrp., Armonk, NY, USA; SPSS, RRID: SCR_002865). Considering the relative low number of animals per group, we have depicted the exact p-values in Fig. 4-6.

\section{Results}

\section{Ureaplasma parvum cultures and detection}

Chronic UP infection in animals inoculated at 80 days of gestation was confirmed by culture of amniotic fluid at the time of subsequent LPS or saline injections by amniocentesis (Fig. 2). No significant differences in the UP levels were observed between the three groups (42UP $1.1 \pm 0.8 \times 106$ CCU/ml; UP\&2LPS 1.1 $\pm 0.8 \times 107$ CCU/ml; UP\&7LPS $9.4 \pm 0.7 \times 106 \mathrm{CCU} / \mathrm{ml}$ ). Cultures of amniotic fluid at time of delivery were positive for UP in all experimentally infected animals except one of the animals of the UP\&7LPS group. No endogenous UP growth was observed in the amniotic fluid of animals that were not inoculated with UP (SAL, 2LPS and 7LPS groups). No UP growth in CSF or plasma was observed for any animal.

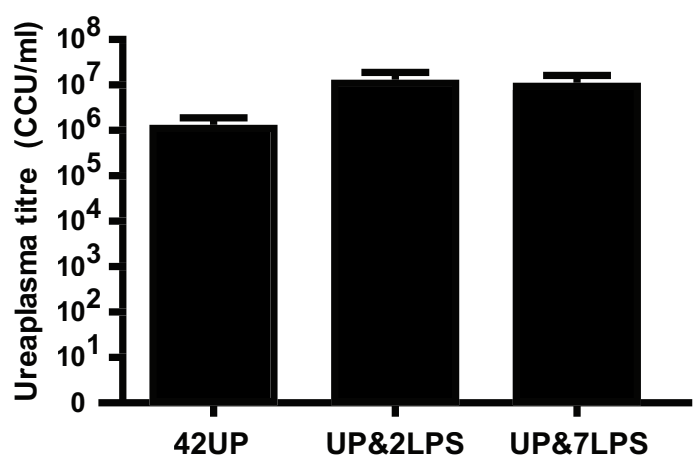

Figure 2. Ureaplasma titer at time of subsequent LPS or saline injection. Establishment of chronic infection was confirmed in amniocentesis samples taken at time of LPS or saline injection. Mean and standard error of the mean are shown for each group (culture titration of viable UP determined in triplicate for each animal). No statistical difference was found by one-way ANOVA testing 


\section{Animal characteristics}

Overall, no sex differences in susceptibility were observed in all readouts. LPS exposure for 2 days decreased the body weight (SAL vs. 2LPS; $\mathrm{p}=0.002$ ) and increased brain-to-body ratio (SAL vs. 2LPS; $\mathrm{p}=$ 0.038) compared to controls (Table 1). These significant changes were not observed in animals that were chronically exposed to UP prior to 2 days of LPS exposure (UP\&2LPS). Moreover, no change in brain weight or brainto-body ratio was observed in animals of the 42UP, 7LPS and UP\&7LPS groups compared to control animals.

\begin{tabular}{lllllll}
\hline & $\begin{array}{l}\text { SAL } \\
(\mathbf{n}=7)\end{array}$ & $\begin{array}{l}\text { 42UP } \\
(\mathbf{n}=6)\end{array}$ & 2LPS (N=5) & 7LPS (N=5) & $\begin{array}{l}\text { UP\&2LPS } \\
(\mathbf{N}=5)\end{array}$ & $\begin{array}{l}\text { UP\&7LPS } \\
(\mathbf{n}=5)\end{array}$ \\
& $3021 \pm 328$ & $2765 \pm 312$ & $2256 \pm 305^{*}$ & $3040 \pm 285$ & $2580 \pm 285$ & $2652 \pm 396$ \\
$\begin{array}{l}\text { Body } \\
\text { weight (g) }\end{array}$ & $52.2 \pm 4.5$ & $50.8 \pm 4.9$ & $46.0 \pm 2.8 \#$ & $51.7 \pm 7.0$ & $47.9 \pm 2.1$ & $51.7 \pm 4.1$ \\
$\begin{array}{l}\text { Brain } \\
\text { weight (g) }\end{array}$ & & $1.9 \pm 0.02$ & $2.1 \pm 0.02^{*}$ & $1.7 \pm 0.02$ & $1.9 \pm 0.02$ & $2.0 \pm 0.02$ \\
$\begin{array}{l}\text { Brain/body } \\
\text { Ratio (\%) }\end{array}$ & $1.7 \pm 0.02$ & & & & \\
\hline
\end{tabular}

Table 1. Animal characteristics. Animal characteristics are expressed as mean $[ \pm S D]$. One-way ANOVA with Dunnett's multiple comparison testing was performed. * $\rho<0.05$ versus control; \# $\rho<0.1$ versus control

\section{Circulatory pro-inflammatory cytokine levels}

Elevated plasma IL- 6 concentrations were found in $50 \%$ (3/6) of the 2 day LPS exposed animals and in 20\% (1/5) of the 42 days UP and 2 days LPS exposed animals when compared to controls (Fig. 3). Plasma IL-6 concentrations in the SAL, 42UP, 7LPS and UP\&7LPS animals were not detectable.

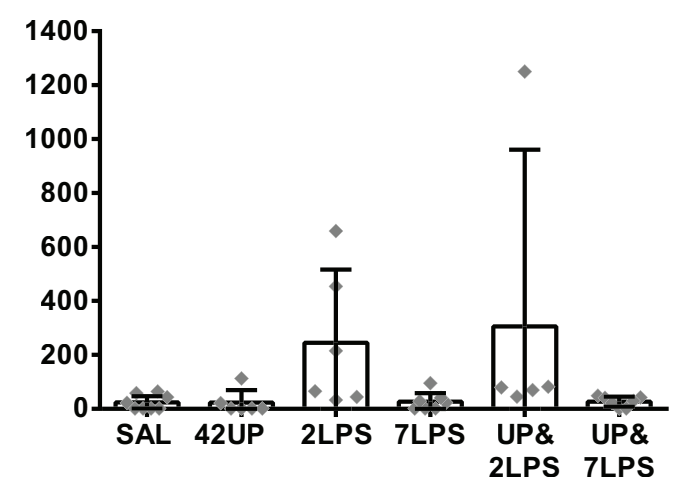

Figure 3. Systemic immune activation was ascertained by measuring circulatory IL-6 concentrations. For statistical analysis, undetectable values were assigned an arbitrary value of $1 \mathrm{pg} /$ $\mathrm{mL}$. No statistical difference was found by one-way ANOVA testing. 


\section{Structural analysis of the brain}

Qualitative analysis of H\&E stained sections revealed increased cell densities in the gyral crest of the white matter which primarily consisted of glial cells. These gliotic foci were most prominent in $3 / 6$ (50\%) of the $2 \mathrm{~d}$ LPS exposed animals. Furthermore, in $1 / 6(16 \%)$ of the $42 \mathrm{~d}$ UP exposed animals and in $1 / 6(16 \%)$ of the 7d LPS exposed animals these gliotic foci were present. Control animals and animals of the UP \& LPS combined groups had mild to no gliotic foci. No evidence of structural changes including intraventricular hemorrhages and cystic lesions were present in any of the experimental groups.

\section{Dual effects of chronic UP infection on cerebral development.}

The neuroinflammatory changes, as indicated by the more pronounced presence of gliotic foci in the 2d LPS exposed animals were further evaluated by cell counts and area fraction analysis of the microglial marker IBA-1 and astrocytic marker GFAP in the cerebral white matter and hippocampus. Chronic intra-amniotic exposure to UP decreased GFAP immunoreactivity (IR) (SAL vs. 42UP; $\mathrm{p}=0.020$ ) and the number of astrocytes (SAL vs. 42UP; $\mathrm{p}=0.100$ ), compared to controls (Fig. 4). IBA-1 IR and IBA-1 positive cell numbers remained unaltered following chronic intra-amniotic UP exposure (Fig. 4). In contrast, acute exposure to LPS increased IBA-1 IR (SAL vs. 2LPS; $p=0.008$ ) and the number of IBA-1 positive cells (SAL vs. 2LPS; $\mathrm{p}=0.036$ ) in the cerebral white matter (Fig. 4). In addition, morphological analysis revealed a higher density of amoeboid microglia present in the white matter after 2 days of LPS exposure (Fig. 4 inserts). However, if the animals were chronically exposed to UP prior to LPS, no IBA-1 IR or IBA-1 positive cell increase was observed in the cerebral white matter at 2 or 7 days post-LPS challenge (Fig. 4d\&f). Equally, LPS administration did return GFAP IR in chronically UP infected animals to control levels (Fig. 4c). This preconditioning effect of UP was also found in the hippocampus in which an increase of IBA-1 IR was found at 2 and 7 days following LPS exposure but not in the groups with pre-exposure to UP (SAL vs. 2LPS; $p=0.002$ and SAL vs. 7LPS; $p=0.000$ ) (data not shown). No changes of GFAP IR or GFA positive cell numbers were found following LPS exposure in the white matter (Fig. 4) and in the hippocampus (data not shown). 

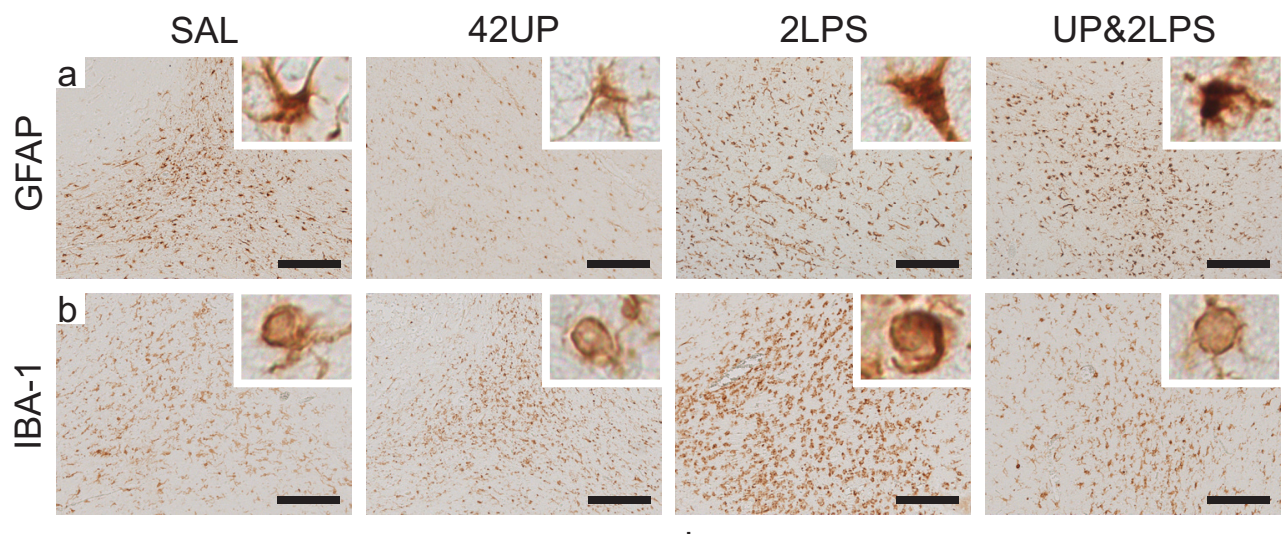

C

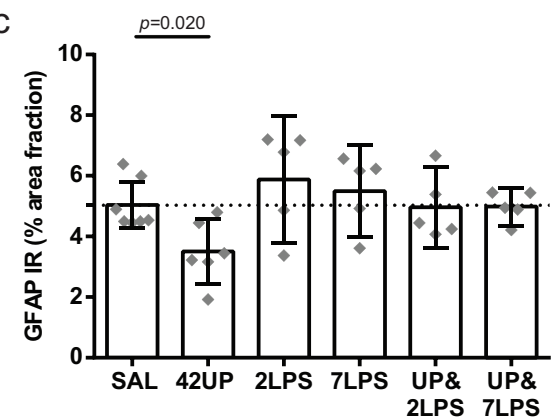

d
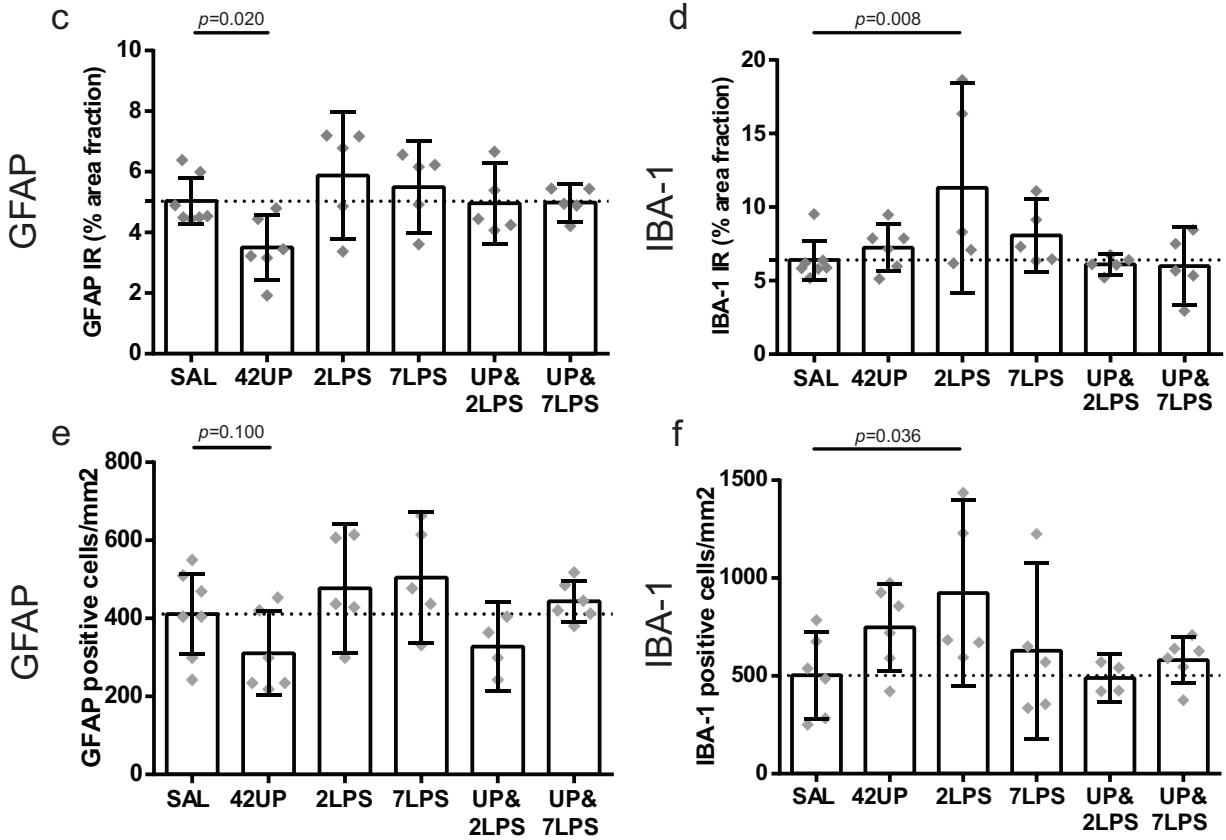

Figure 4. Intra-amniotic exposure to Ureaplasma parvum (UP) induces a decrease of astrocytes and preconditions the fetal brain against re-exposure to lipopolysaccharide (LPS). A significant decrease $\left({ }^{*}, \mathrm{p}<0.05\right)$ of the area fraction of GFAP immunoreactivity (IR) was observed in animals of the chronic UP group (42UP) compared to controls (SAL) (SAL vs. 42UP; $p=0.020$ ) (a \& c). GFAP positive cells tended to be decreased following chronic UP exposure (SAL vs. 42UP; $p=0.100$ ) (e). No changes in GFAP IR were found in animals of the LPS exposed groups regardless of the presence or absence of UP pre-exposure. Significant increase $\left({ }^{*}, \mathrm{p}<0.05\right)$ of the area fraction of IBA-1 IR and IBA-1 positive cells was observed in animals of the $2 \mathrm{~d}$ LPS group (2LPS) (SAL vs. 2LPS; $\mathrm{p}=0.008$ and $\mathrm{p}=0.036$ respectively) which was prevented by pre-exposure to UP (UP\&2LPS) (b \& d \& f). No changes of IBA-1 IR and cell numbers were found in animals of the $7 \mathrm{~d}$ LPS group (SAL vs. 7LPS; $\mathrm{p}=0.342$ and $\mathrm{p}=1.00$ respectively) ( $\mathrm{d} \& \mathrm{f}$ ). Representative histological figures of the GFAP positive astrocytes and IBA-1 positive microglia are shown in (a) and (b) respectively. Morphological analysis showed a higher density of amoeboid microglia present in the white matter after 2 days of LPS exposure (b, inserts). Figures of animals of the $7 \mathrm{~d}$ LPS group (7LPS) and $42 \mathrm{~d}$ UP and $7 \mathrm{~d}$ LPS group (UP\&7LPS) are not depicted. GFAP and IBA-1 IR are represented in the graphs as mean $\%$ area fraction $\pm 95 \%$ CI. Images taken at 100x magnification (insert at 400x magnification), scale bar $=200 \mu \mathrm{m}$ 
Since the choroid plexus is primarily dominated by macrophages, T-lymphocytes and dendritic cells for continuous immune surveillance, and the resolution of cerebral inflammation [26] we analyzed the distribution of CD68+ macrophages, CD3+ lymphocytes and MPO+ neutrophils within the choroid plexus. MPO-positive cells tended to be increased following $7 \mathrm{~d}$ LPS exposure compared to control animals and this increase was prevented by pre-exposure to $42 \mathrm{~d}$ UP (SAL vs. 7LPS; $\mathrm{p}=0.086$ ) (Tabel 2). In line, this increase in MPO-positive cells at 7d post LPS exposure was accompanied by a decrease in IBA- 1 IR in the cerebral white matter compared to $2 \mathrm{~d}$ LPS exposed animals. No differences in CD68 and CD3- positive cells were found in the choroid plexus. No CD3- and MPO-positive cells were detected in the brain parenchyma.

\begin{tabular}{lllllll}
\hline cells/mm & SAL (n=7) & 42UP (n=6) & 2LPS (N=5) & 7LPS (N=5) & $\begin{array}{l}\text { UP\&2LPS } \\
(\mathbf{N}=5)\end{array}$ & $\begin{array}{l}\text { UP\&7LPS } \\
(\mathbf{n}=5)\end{array}$ \\
$\begin{array}{l}\text { MPO+ cells } \\
3\end{array}$ & $3.31 \pm 2.72$ & $6.80 \pm 10.29$ & $8.58 \pm 2.84$ & $14.66 \pm 8.96^{\sharp}$ & $1.06 \pm 0.87$ & $5.68 \pm 9.36$ \\
$\begin{array}{l}\text { CD68+ } \\
\text { cells }\end{array}$ & $67.99 \pm 47.40$ & $48.45 \pm 33.92$ & $69.91 \pm 51.64$ & $80.62 \pm 32.07$ & $64.49 \pm 49.05$ & $59.31 \pm 40.18$ \\
$\begin{array}{l}\text { CD3+ } \\
\text { cells }\end{array}$ & $28.98 \pm 24.96$ & $11.14 \pm 7.21$ & $30.82 \pm 34.98$ & $47.24 \pm 23.73$ & $26.11 \pm 35.25$ & $15.92 \pm 19.32$ \\
\hline
\end{tabular}

Table 2. Immune cells present in the choroid plexus. Cell counts of MPO+, CD68+ and CD3+ cells in the choroid plexus are expressed as cells/mm2 $[ \pm S D$ ). One-way ANOVA with Dunnett's multiple comparison testing was performed. ${ }^{*} \rho<0.05$ versus control; ${ }^{*} \rho<0.1$ versus control

White matter injury was studied by assessing the densities of mature (MBP) and overall (Olig2) oligodendrocytes in the cerebral white matter. An apparent increase in Olig2 positive cells was found for all groups relative to control levels; however, this only reached significance for chronically UP-infected animals (SAL vs. 42UP; $\mathrm{p}=0.012$ ) and animals exposed to LPS for 2 days (SAL vs. 2LPS; $\mathrm{p}=0.037$ ) (SAL vs. 7LPS; $\mathrm{p}=0.211$, SAL vs. 42UP\&2LPS; $p=0.558$, SAL vs. 42UP\&7LPS; $p=0.467$ ) (Fig. 5c). In addition, MBP IR tended to be decreased at 42d following UP exposure (SAL vs. 42UP; $p=0.097$ ) (Fig. 5). Short term LPS exposure for 2 days resulted in a decrease of MBP IR within regions of overt microgliosis (SAL vs. 2LPS; $\mathrm{p}=$ 0.001 ) which was prevented by pre-exposure to UP. At 7d of LPS exposure no changes in MBP IR were found (2LPS vs. 7LPS; $p=0.000$ ). 
We analyzed 5-mc and 5-hmc integrated density as epigenetic markers for DNA-methylation in the dentate gyrus of the hippocampus. We focused our analysis on the dentate gyrus since this is the region in the hippocampus were neurogenesis takes place and DNA methylation and demethylation are important contributors to this process [27, 28]. Both short term LPS exposure as well as chronic UP exposure resulted in an increase of the gene repression marker 5-mc integrated density compared to controls (SAL vs. 2LPS; $\mathrm{p}=0.008$ and SAL vs. 7LPS; $\mathrm{p}=0.002$ and SAL vs. 42UP; $\mathrm{p}=0.008$ ) (Fig. 6). The increase in 5-mc following LPS exposure tended to be prevented by pre-exposure to UP.

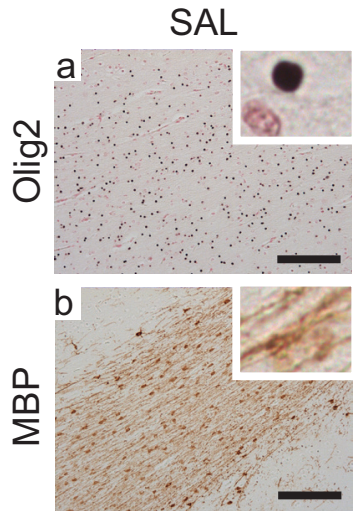

C

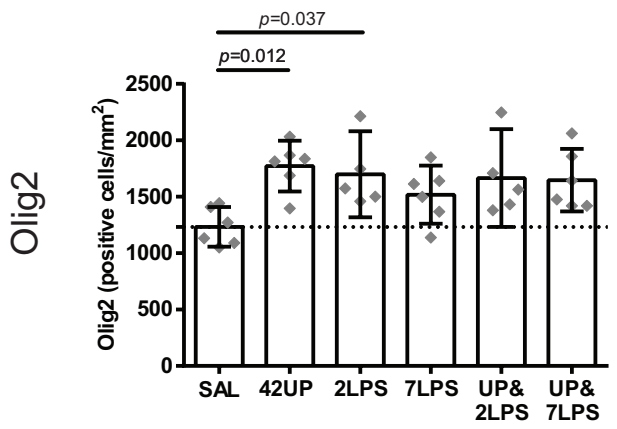

42UP
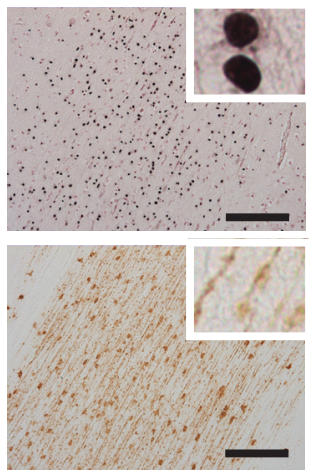

d

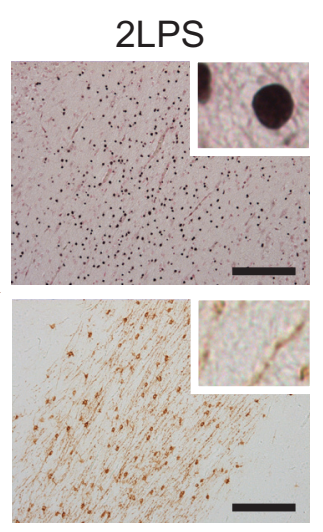

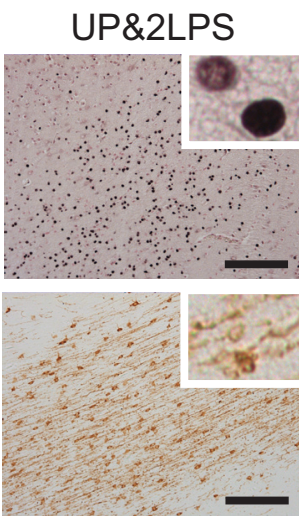

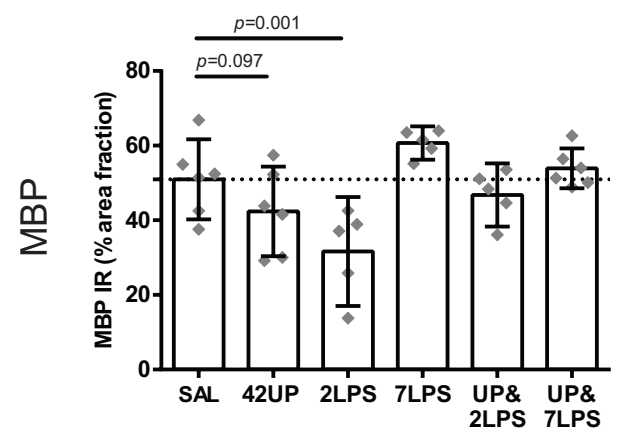

Figure 5. Intra-amniotic exposure to Ureaplasma parvum (UP) induces changes in white matter development and preconditions the fetal brain upon re-exposure to a second inflammatory hit with lipopolysaccharide (LPS). Significant increase $\left({ }^{*}, \mathrm{p}<0.05\right)$ of Olig2 positive cells was observed in animals of the chronic UP group (SAL vs. 42UP; $\mathrm{p}=0.012$ ) and of the 2d LPS group (SAL vs. 2 LPS; $\mathrm{p}=$ 0.037 ) compared to controls ( $\mathrm{b} \& \mathrm{c}$ ). This increase of Olig2 positive cells was accompanied by a decrease in MBP (SAL vs. 42UP; $\mathrm{p}=0.097$ and SAL vs. 2LPS; $\mathrm{p}=0.001$ ) (a \& d). This decrease of MBP IR was prevented in the short term LPS exposed animals that were pre-exposed to UP. At 7d of LPS exposure no changes in MBP IR were found (2LPS vs. 7LPS; $\mathrm{p}=0.000$ ). Olig2 is represented as mean positive cells/ $\mathrm{mm} 2 \pm 95 \% \mathrm{CI}$ and MBP IR is represented in the graphs as mean \% area fraction $\pm 95 \%$ CI. Images taken at 100x magnification (insert at 400x magnification), scale bar $=200 \mu \mathrm{m}$ 
An increase in transcription activation marker 5-hmc integrated density was only found in $2 \mathrm{~d}$ LPS exposed animals compared to controls which was prevented by pre-exposure to UP (SAL vs. 2LPS; $\mathrm{p}=0.000 ; 2 \mathrm{LPS}$ vs. 7LPS; $\mathrm{p}=$ 0.000) (Fig. 6).

We demonstrated with MALDI-MSI unique regional differences of the lipid composition in the preterm ovine brain between animals from the control, 42UP, 2LPS and UP\&LPS group. Fig. 7 shows the reconstructed image that represents the molecular differences of lipids between white (red area) and grey matter (green area). The lipid composition, characteristic for healthy white and grey matter of the preterm brain was not altered following chronic UP exposure (Fig. 7a-c). In particular, in the white matter of control and 42UP animals, typical tentative assigned $\mathrm{m} / \mathrm{z}$ values of different phosphocholine (PC) species such as m/z 782.5 PC 34:1+Na+, m/z 810.5 PC 36:1+Na+ or sphingomyelin m/z $725.5 \mathrm{SM} 34: 1+\mathrm{Na}+$ are found which are known to be representative for the white matter [29, 30] (Fig. 7c). m/z 756.5 PC 32:0+Na+ which is a characteristic grey matter lipid [31], were detected in the grey matter of control and 42UP animals, demonstrating that control and chronic UP exposed animals had a similar and constitutive lipid profile. Mosaic PCA-images demonstrated that short-term LPS exposure resulted in lipid accumulation in the white matter and diffusion of white matter specific molecular patterns into the grey matter and vice versa (Fig. 7d-i; Principal component 4 \& 6). These changes were reduced when LPS exposure was preceded by 42 days of UP infection (Fig. $7 \mathrm{e} \& \mathrm{~h}$ ). In particular, the abundance of the white matter specific component $\mathrm{m} / \mathrm{z} 725.5$ in the grey matter of LPS exposed animals was not present in the grey matter of control or UP animals (Fig. 7g-i; negative spectrum of principal component 6). Whereas, $\mathrm{m} / \mathrm{z} 734.5 \mathrm{PC} 32: 0+\mathrm{H}+$ was accumulated in the grey matter of LPS animals (negative spectrum of principal component 4), and increased in the white matter (positive spectrum of principal component 6). In addition, principal component 6 showed that other peaks such as $\mathrm{m} / \mathrm{z} 760 \mathrm{PC}$ 34:1+H+ accumulate in the white matter at $2 \mathrm{~d}$ of LPS exposure which did not correspond with the pattern seen in our controls and those from others [29] in which these peaks were evenly distributed. 

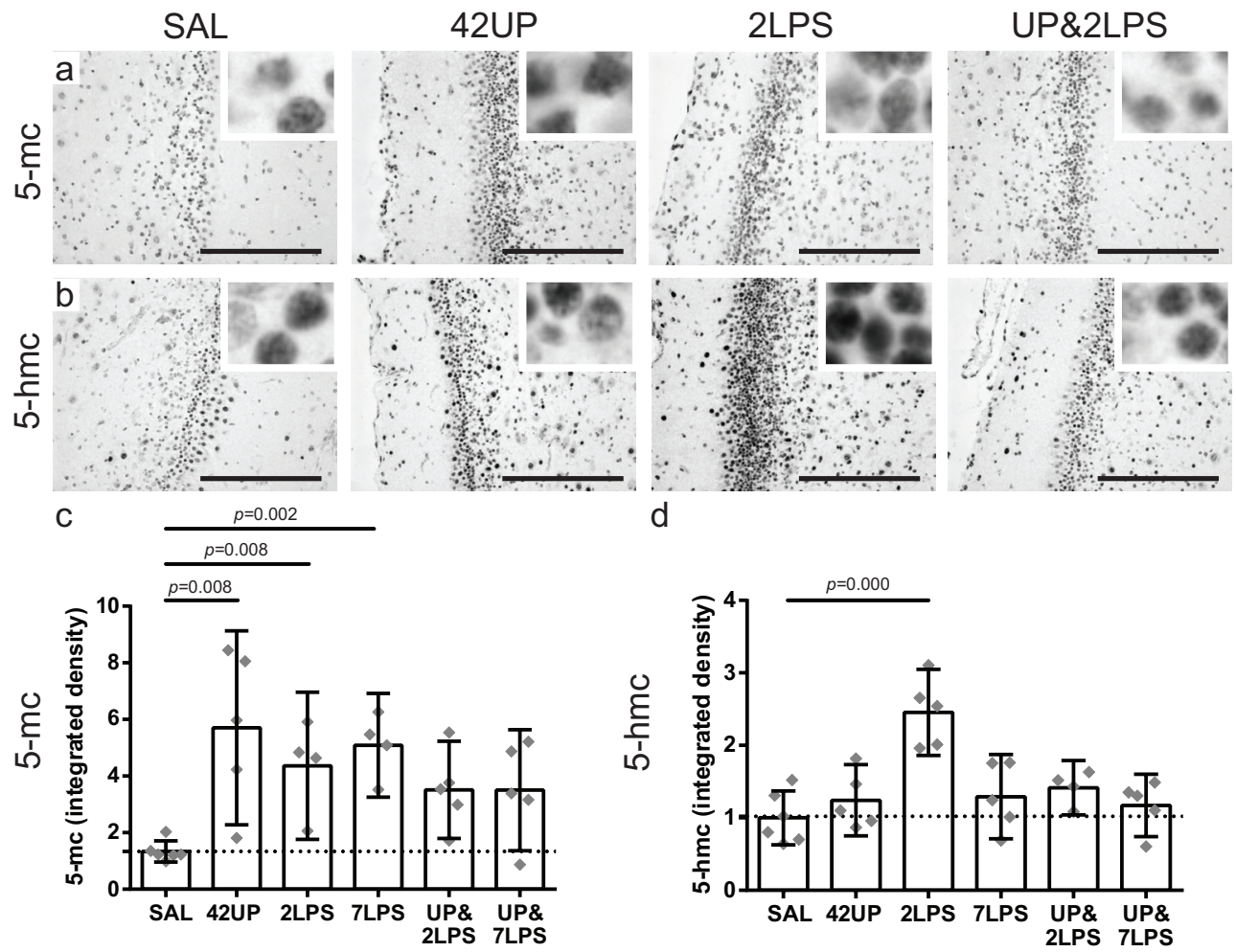

Figure 6. Changes of the epigenetic markers 5-mc and 5-hmc following intra-amniotic exposure to Ureaplasma parvum (UP) and short-term exposure to lipopolysaccharide (LPS) in the dentate gyrus of the hippocampus. Significant increase $\left({ }^{*}, \mathrm{p}<0.05\right)$ of the gene repression marker 5 -mc IR was observed in short term LPS exposed animals and chronic UP exposed animals when compared to controls (SAL vs. 2LPS; $\mathrm{p}=0.008$ and SAL vs. 7LPS; $\mathrm{p}=0.002$ and SAL vs. 42UP; $\mathrm{p}=0.008$ ) (a \& c). Significant increase $\left({ }^{*}, \mathrm{p}<0.05\right)$ in transcription activation marker 5 -hmc IR was restricted to the 2d LPS group compared to controls (SAL vs. 2LPS; $p=0.000 ; 2 L P S$ vs. 7LPS; $p=0.000$ ), which was prevented by pre-exposure to UP. 5-mc and 5-hmc are represented in the graphs as mean integrated density $\pm 95 \%$ CI. The integrated density was calculated by multiplying the area fraction and gray intensity measurements. Accumulation of lipids and changes in the white and grey matter lipid profile following acute LPS exposure is prevented by pre-exposure to UP. Values were normalized to the data of the control group. Images taken at 200x magnification (insert at 400x magnification), scale bar $=200$ $\mu \mathrm{m}$ (insert at $400 \mathrm{x}$ magnification), scale bar $=200 \mu \mathrm{m}$ 
> Figure 7. Matrix assisted laser desorption ionization mass spectrometry imaging (MALDI-MSI) performed in positive ion mode followed by principal component analysis (PCA). a shows the reconstructed image that represents the molecular differences of lipids between white (red area) and grey matter (green area) of control and UP animals. PCA 3 demonstrates similar lipid compositions of white and grey matter of control and UP animals (a-c). In particular, phosphocholine (PC) species $\mathrm{m} / \mathrm{z}$ 782.5 PC 34:1+Na+, m/z 810.5 PC 36:1+Na+ or sphingomyelin m/z 725.5 SM 34:1+Na+ are present in white matter (positive spectrum principal component 3) whereas $\mathrm{PC} \mathrm{m} / \mathrm{z} 756.5$ 32:0+Na+ is present in grey matter of control and 42UP animals (negative spectrum principal component 3 ). Short-term LPS exposure results in lipid accumulation in the white matter as illustrated by an increased intensity of the white matter related peaks, especially m/z 725.5 (d \& g) (positive spectrum of principal component 4). In addition, a shift of white and grey matter specific molecular patterns is observed at $2 \mathrm{~d}$ LPS exposure illustrated by more white matter specific lipids present in the grey matter such as $\mathrm{m} / \mathrm{z} 725.5$ (d, negative spectrum of principal component 6); and grey matter specific lipids into the white matter such as $\mathrm{m} / \mathrm{z}$ $734.5 \mathrm{PC} 32: 0+\mathrm{H}+$ and $\mathrm{m} / \mathrm{z} 760 \mathrm{PC} 34: 1+\mathrm{H}+$ (g, positive spectrum of principal component 6). These changes were prevented by pre-exposure to UP (e \& h)

Other lipid species including phosphatidylinositols (PI) and sulfatide (SF) can be identified using the negative ion mode in MALDI-MSI. Regions of interest corresponding to the white matter were selected based on the results with the positive ion mode. The DF1 (Fig. 8a) reveals that the highest differences are observed between the control (negative scores) and LPS group (positive scores). The UP\&LPS group had negative scores and therefore possessed a molecular composition more similar to the control group. The DF1 spectrum (Fig. 8b) shows the lipid composition of the white matter of the 2 days of LPS exposed animals. This spectrum showed that LPS exposure reduced the amount of tentative assigned sulfatide (SF) species such as m/z 806.5 SF 18:0-H-, m/z 888.6 SF 24:1-H-, m/z 890.6 SF 24:0-H-, which were described as lipids characteristic of white matter [29]. These species were mainly present in the white matter of control, UP and UP\&LPS groups (spectrum not shown) whereas m/z 885.5 PI 38:4-H- or m/z 718.6 PC 31:0-H- were representative of the white matter in animals of the 2LPS group (fig.8b). 

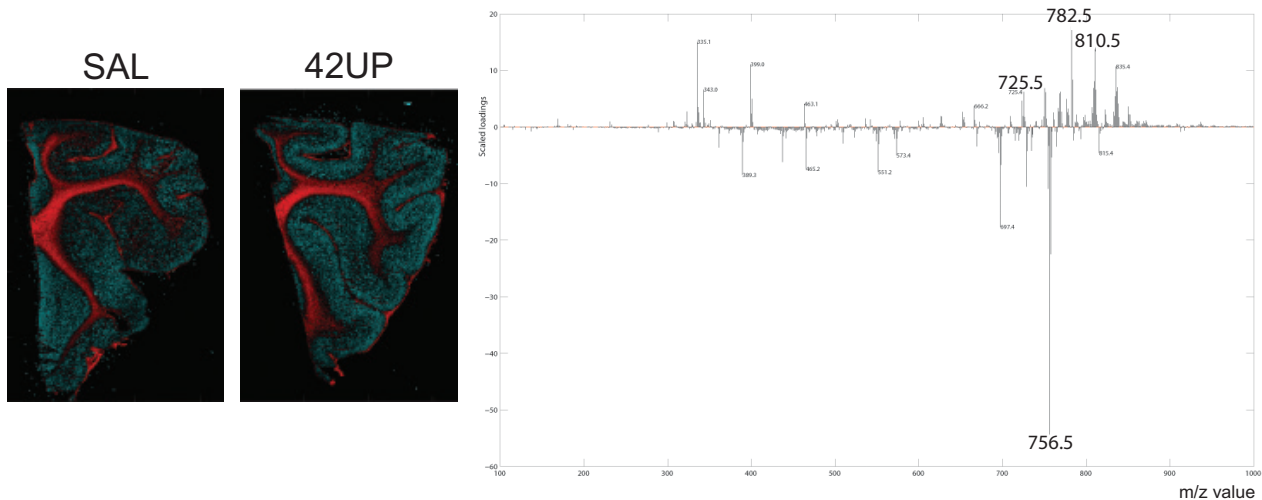

d

e

f

PRINCIPAL COMPONENT 4

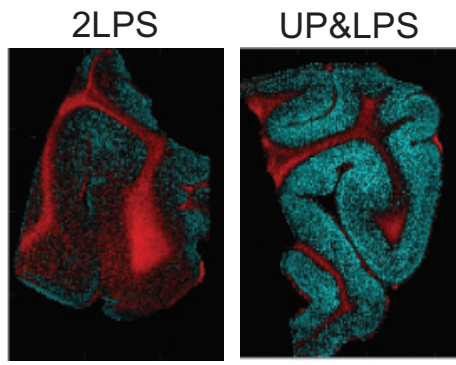

g

h

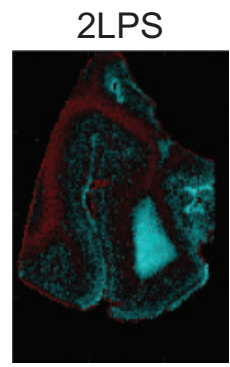

UP\&LPS
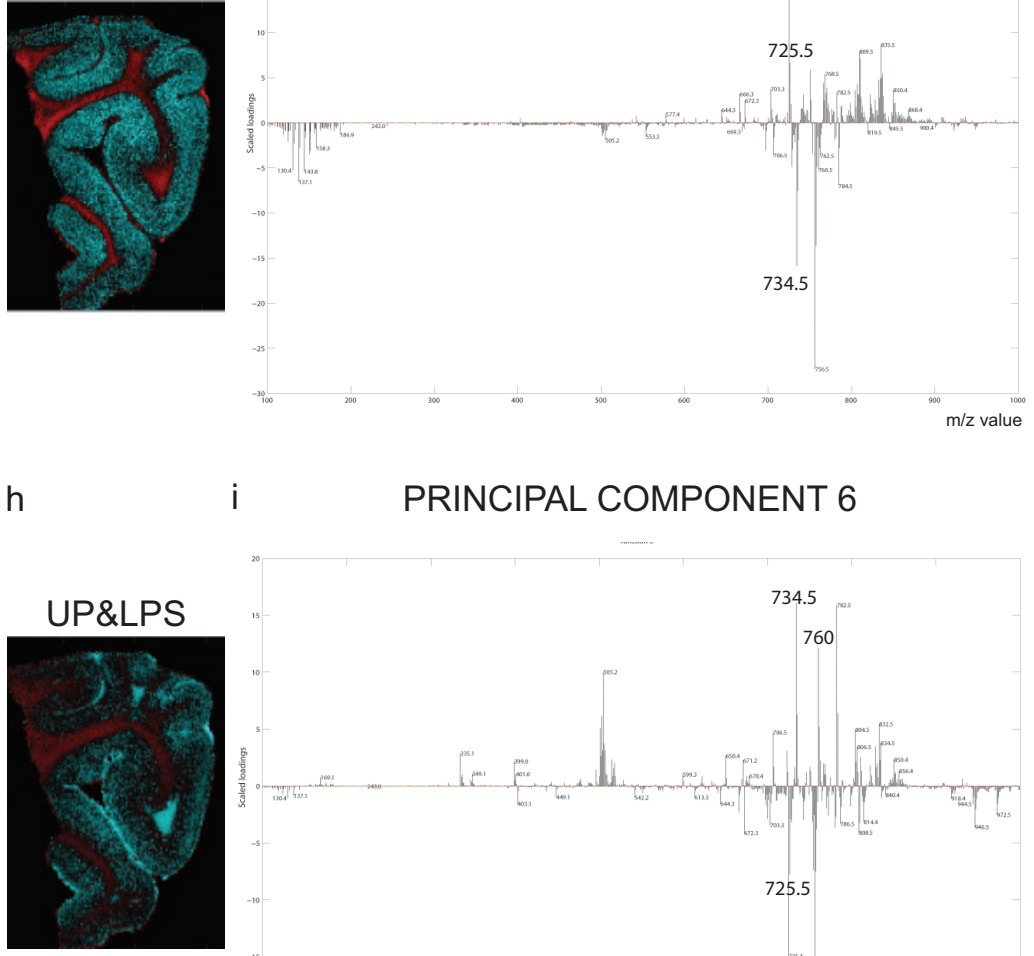

\section{PRINCIPAL COMPONENT 6}


a

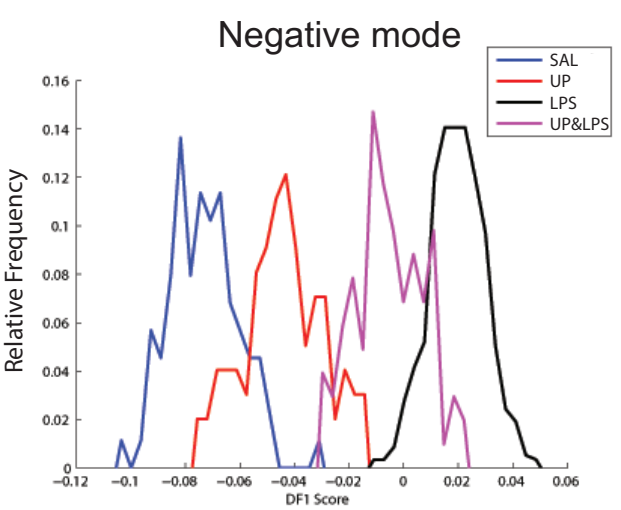

b

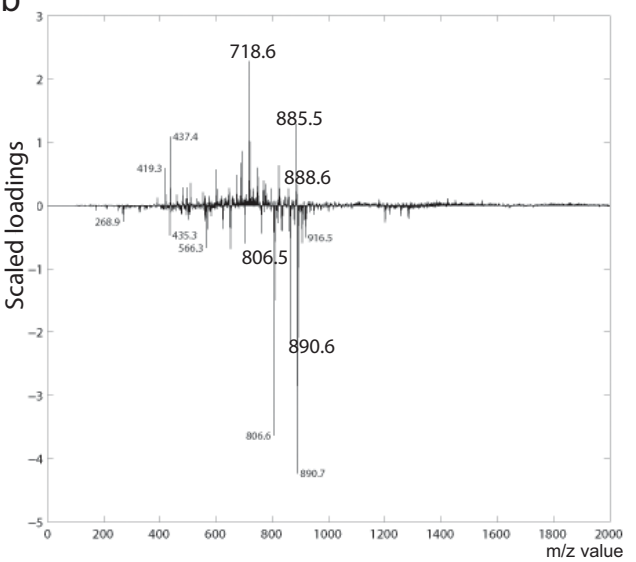

Figure 8. Matrix assisted laser desorption ionization mass spectrometry imaging (MALDI-MSI) performed in negative ion mode followed by PCA. The DF1 (a) revealed that the highest differences were observed between the control (negative scores) and LPS group (positive scores). Both 42UP and UP\&LPS groups had negative scores which were comparable to the control group. The DF1 spectrum of the 2 days of LPS exposed animals (b) showed that LPS infection reduced the amount of sulfatide (SF) species such as m/z 806.5 SF 18:0-H-, m/z 888.6 SF 24:1-H-, m/z 890.6 SF 24:0-H-. These species were mainly present in the white matter of control, UP and UP\&LPS groups (spectrum not shown) whereas $\mathrm{m} / \mathrm{z}$ 885.5 PI 38:4-H- or m/z 718.6 PC 31:0-H- were representative of the white matter in animals of the 2LPS group (b) 


\section{Discussion}

Chronic intra-amniotic Ureaplasma parvum (UP) exposure decreased GFAP immunoreactivity (IR) and increased Olig2 positive cells and 5 -methylcytosine (5-mc) IR in the brain. These changes have potential clinical implications postnatally.

The observed decrease of GFAP IR and number of astrocytes (GFAP+ cells) at $42 \mathrm{~d}$ of UP exposure is important since these cells possess several essential functions in brain development including regulation of the extracellular glutamate homeostasis, providing structural and metabolic support to surrounding cells (e.g. oligodend rocytes) and modulate neuronal connections [32]. Changes in astrocyte function or density result in altered neurological outcomes. In particular, altered astrocyte protein expression (GFAP) and disrupted astrocyte maturation have been implicated in the pathogenesis of neurodevelopmental disorders such as autism and cerebral palsy [33, 34]. Moreover, Sharma et al. [35] showed that LPS injection in the spinal cord of rodents decreased astrocytes which was followed by hypomyelination. This suggests that white matter injury, a hallmark of preterm brain injury, can still occur in these fetuses considering the loss of GFAP IR at 42d post UP exposure. Collectively, the astrocyte cell and protein loss in our study indicates that chronic UP exposure during the second trimester of gestation predisposes to brain pathologies that are often seen in newborns.

Second, the increase of oligodendrocyte lineage cells, as seen following $42 \mathrm{~d}$ of UP exposure might indicate replenishment of oligodendrocytes upon initial lossin the acute phase following UP exposure [36]. Importantly, UP was administered at $80 \mathrm{~d}$ of gestation which is the premyelinating stage of brain development with abundant vulnerable immature pre-oligodendrocytes, sensitive to glutamate receptor induced injury [37]. Interestingly, the mature oligodendrocytes tended to be decreased following chronic UP exposure. Given these combined findings of increased Olig2+ cell numbers and reduced $\mathrm{MBP}+\mathrm{IR}$, it iss tempting to speculate that this indicates a 
maturation arrest of oligodendrocyte progenitor cells, a key feature of white matter injury of preterms [38-40]. This oligodendrocyte maturation arrest can be linked to the decreased astrocytes that we found in this study. Astrocytes are essential contributors to extracellular glutamate homeostasis which will be disturbed by a loss of astrocytes [32]. Since immature oligodendrocytes are particularly vulnerable to glutamate receptor induced injury, this can lead to oligodendrocyte injury [37]. In addition, oligodendrocytes rely on astrocytes for their metabolic support via gap junctions [41]. Failure of metabolic support for oligodendrocytes following astrocyte loss results in energy failure and eventually maturation arrest or death. Alternatively, it is tempting to speculate that oligodendrocyte maturation arrest may be connected to the apparent increase of the DNA-methylation marker 5-mc at 42 days of UP exposure, which is a very important repressor of gene transcription [42]. This theory is supported by several reports stating that changes in epigenetic regulatory mechanisms contribute to disturbed maturation and differentiation of immature oligodendrocytes [43-45]. Moreover, inflammation induced epigenetic changes during early development can cause substantial lasting neurodevelopmental impairments later in life [46, 47]. Altogether, these data offer mechanistic insight in the association between intra-amniotic UP exposure and the increased incidence of adverse neurodevelopmental outcomes postnatally.

Interestingly, the cerebral phenotype following short term LPS exposure was remarkably different when compared to chronic UP exposure. In particular, we demonstrated that short term LPS exposureinduced a rapid and temporal increase of the number of microglia and decreased myelin immunoreactivity, reflecting diffuse cerebral inflammation with hypomyelination. Microglia are important for inflammatory perinatal brain injury [43]. Aberrant or excessive microgliosis can be detrimental for the immature brain resulting in white matter injury [14], which corresponds to the loss of myelin that we found in our study. The cerebral inflammatory response following LPS in this study seems to be temporal since no increase in microglial density was found following 7d of LPS exposure. This dynamic response of activated microglia is consistent with distinct phases of cerebral inflammation [48] and can be explained by the presence of neutrophils in the choroid plexus, which are known to be important to the resolution of cerebral inflammation [26]. However, our immunohistochemical analysis does not rule out the possibility that phenotypic conversion of microglia might be induced following short term LPS or chronic UP exposure, which might influence the cerebral immune response. 
Although such short time UP effects were not investigated in our model, in a study performed by Normann et al. [11] in rodents short term UP exposure during early pregnancy resulted in increased microglial density and decreased myelin basic protein density in the fetal brain. This cerebral phenotype is consistent with our data following short term LPS exposure indicating that timing and not the inflammatory trigger is more important in the neurological outcome of the fetal brain.

Besides the DNA-methylation marker 5-mc, the hydroxylated product and transcription activation marker 5-hmc was increased following $2 \mathrm{~d}$ of LPS exposure. 5-hmc is very important for proper neurodevelopment and 5 -hmc is altered in the umbilical cord of babies born after pre-eclampsia and gestational diabetes mellitus [49] and in the hippocampus of 7-week old mice exposed to non-infectious stress [50]. In addition, 5-hmc alterations are associated with severe neurodevelopmental disorders such as Rett syndrome which is caused by mutations in the MeCP2 gene that encodes for proteins that directly bind to methylated DNA domains [51]. Therefore, the alterations we found in epigenetic markers following acute LPS exposure might explain, at least in part, the association between chorioamnionitis and the development of psychopathology later in life [52]. Since epigenetic changes are reversible, these findings provide new therapeutic targets to prevent long lasting neurodevelopmental morbidities following prenatal stress [17].

In addition, our lipid data provide supporting evidence that short-term LPS exposure results into lipid accumulation and "diseased" lipid patterns in the preterm brain. Such lipid accumulation in the brain is associated with severe neurological damage and altered brain functions [20]. In addition, we show a relative decrease of the myelin-specific sphingolipids in the white matter of the LPS exposed animals, which confirms and extends our findings concerning the loss of MBP in these animals. The abundance of phosphatidylinositols (PI) following $2 \mathrm{~d}$ of LPS exposure was primarily seen within the region of increased IBA-1 IR. In line, the phosphorylated form of PI, phosphoinositide, is known to be upregulated in microglia and contributes to activation of microglia following ischemia [53].

Third, we found that chronic intra-amniotic UP exposure prevented an increase of IBA-1 IR and IBA-1+ cells, 5-hmc IR, lipid profile changes 
and a decrease of MBP IR upon a second inflammatory hit with LPS. This phenotype, also referred to as 'preconditioning', has been previously described in animal models in which pre-exposure to inflammation induced by LPS renders the brain less susceptible to a second hypoxic-ischemic insult, thereby resulting in less brain injury $[54,55]$. This preconditioning effect of chronic UP exposure could be explained by work from Cao et al. [56] which showed in pregnant sheep that microglia once activated in vivo by intra-amniotic LPS exposure, display diminished inflammatory responses following re-exposure to LPS. Moreover, they state that the memory acquired by microglia upon the first exposure to inflammation might be mediated by epigenetic regulatory processes [56]. Although this hypothesis needs to be tested in future studies, it is noteworthy that changes in the global level of 5-hmC and 5-mC were observed in our study following acute LPS exposure that was prevented by chronic UP exposure. Clearly, long term protection after inflammation induced preconditioning needs to be confirmed in a longitudinal study, but it is considered to be permanent since structural and functional protection up to 8 weeks was established following hypoxic preconditioning in a neonatal rodent model [57].

One important limitation of a large animal study is the relative low number of animals per group. Given the relative small animal numbers per group, we report actual $p$-values and tend to interpret $p$-values between 0.05 and 0.1 as biologically relevant. This assumption will decrease the chance of a false negative finding but increases the chance that one of these differences is a false positive result.

In this double-hit study, in which sequential different infectious hits were tested, we show that microbial interactions, the moment of onset and duration of these potential injurious triggers determine the neurological outcome. These findings seem to be an important explanation for the diversity of neurological outcomes associated with intra-amniotic UP exposure. Altogether, these data emphasize that an accurate history of infections during pregnancy is essential to guide neonatal management which warrants the need for biomarkers to diagnose antenatal infections. 


\section{References}

1. Hagberg H, Gressens P, Mallard C. Inflammation during fetal and neonatal life: implications for neurologic and neuropsychiatric disease in children and adults. Annals of neurology. 2012;71(4):444-57. doi:10.1002/ana.22620.

2. Strunk $T$, Inder $T$, Wang $X$, Burgner $D$, Mallard $C$, Levy O. Infection-induced inflammation and cerebral injury in preterm infants. Lancet Infect Dis. 2014;14(8):75162. doi:10.1016/S1473-3099(14)70710-8.

3. Rovira N, Alarcon A, Iriondo M, Ibanez M, Poo P, Cusi V et al. Impact of histological chorioamnionitis, funisitis and clinical chorioamnionitis on neurodevelopmental outcome of preterm infants. Early human development. 2011;87(4):253-7. doi:10.1016/j. earlhumdev.2011.01.024.

4. Kuypers E, Ophelders D, Jellema RK, Kunzmann S, Gavilanes AW, Kramer BW. White matter injury following fetal inflammatory response syndrome induced by chorioamnionitis and fetal sepsis: lessons from experimental ovine models. Early human development. 2012;88(12):931-6. doi:10.1016/j.earlhumdev.2012.09.011.

5. Sweeney EL, Kallapur SG, Gisslen T, Lambers DS, Chougnet CA, Stephenson SA et al. Placental Infection With Ureaplasma species Is Associated With Histologic Chorioamnionitis and Adverse Outcomes in Moderately Preterm and Late-Preterm Infants. The Journal of infectious diseases. 2016;213(8):1340-7. doi:10.1093/infdis/jiv587.

6. Viscardi RM. Ureaplasma species: role in diseases of prematurity. Clinics in perinatology. 2010;37(2):393-409. doi:10.1016/j.clp.2009.12.003.

7. Viscardi RM. Ureaplasma species: role in neonatal morbidities and outcomes. Archives of disease in childhood Fetal and neonatal edition. 2014;99(1):F87-92. doi:10.1136/ archdischild-2012-303351.

8. Glaser K, Speer CP. Neonatal CNS infection and inflammation caused by Ureaplasma species: rare or relevant? Expert Rev Anti Infect Ther. 2015;13(2):233-48. doi:10.1586/14 787210.2015.999670.

9. Kasper DC, Mechtler TP, Bohm J, Petricevic L, Gleiss A, Spergser J et al. In utero exposure to Ureaplasma spp. is associated with increased rate of bronchopulmonary dysplasia and intraventricular hemorrhage in preterm infants. J Perinat Med. 2011;39(3):331-6. doi:10.1515/JPM.2011.022.

10. Berger A, Witt A, Haiden N, Kaider A, Klebermasz K, Fuiko R et al. Intrauterine infection with 
11. species is associated with adverse neuromotor outcome at 1 and 2 years adjusted age in preterm infants. J Perinat Med. 2009;37(1):72-8. doi:10.1515/JPM.2009.016.

12. Normann E, Lacaze-Masmonteil T, Eaton F, Schwendimann L, Gressens P, Thebaud B. A novel mouse model of Ureaplasma-induced perinatal inflammation: effects on lung and brain injury. Pediatric research. 2009;65(4):430-6. doi:10.1203/PDR.0b013e31819984ce.

13. Goldenberg RL, Andrews WW, Goepfert AR, Faye-Petersen O, Cliver SP, Carlo WA et al. The Alabama Preterm Birth Study: umbilical cord blood Ureaplasma urealyticum and Mycoplasma hominis cultures in very preterm newborn infants. American journal of obstetrics and gynecology. 2008;198(1):43 e1-5. doi:10.1016/j.ajog.2007.07.033.

14. Kirchner L, Helmer H, Heinze G, Wald M, Brunbauer M, Weninger M et al. Amnionitis with Ureaplasma urealyticum or other microbes leads to increased morbidity and prolonged hospitalization in very low birth weight infants. Eur J Obstet Gynecol Reprod Biol. 2007;134(1):44-50. doi:10.1016/j.ejogrb.2006.09.013.

15. Jin C, Londono I, Mallard C, Lodygensky GA. New means to assess neonatal inflammatory brain injury. Journal of neuroinflammation. 2015;12:180. doi:10.1186/ s12974-015-0397-2.

16. Hagberg H, Dammann O, Mallard C, Leviton A. Preconditioning and the developing brain. Semin Perinatol. 2004;28(6):389-95.

17. Fleiss B, Tann CJ, Degos V, Sigaut S, Van Steenwinckel J, Schang AL et al. Inflammationinduced sensitization of the brain in term infants. Developmental medicine and child neurology. 2015;57 Suppl 3:17-28. doi:10.1111/dmcn.12723.

18. Gao Q, Tang J, Chen J, Jiang L, Zhu X, Xu Z. Epigenetic code and potential epigeneticbased therapies against chronic diseases in developmental origins. Drug discovery today. 2014;19(11):1744-50. doi:10.1016/j.drudis.2014.05.004.

19. Liu Y, Hoyo C, Murphy S, Huang Z, Overcash F, Thompson J et al. DNA methylation at imprint regulatory regions in preterm birth and infection. American journal of obstetrics and gynecology. 2013;208(5):395 e1-7. doi:10.1016/j.ajog.2013.02.006.

20. Farooqui AA, Horrocks LA, Farooqui T. Glycerophospholipids in brain: their metabolism, incorporation into membranes, functions, and involvement in neurological disorders. Chemistry and physics of lipids. 2000;106(1):1-29.

21. Adibhatla RM, Hatcher JF. Role of Lipids in Brain Injury and Diseases. Future lipidology. 2007;2(4):403-22. doi:10.2217/17460875.2.4.403.

22. Miura Y, Payne MS, Keelan JA, Noe A, Carter S, Watts R et al. Maternal intravenous treatment with either azithromycin or solithromycin clears Ureaplasma parvum from the amniotic fluid in an ovine model of intrauterine infection. Antimicrobial agents and chemotherapy. 2014;58(9):5413-20. doi:10.1128/AAC.03187-14.

23. Gavilanes AW, Strackx E, Kramer BW, Gantert M, Van den Hove D, Steinbusch H et al. Chorioamnionitis induced by intraamniotic lipopolysaccharide resulted in an intervaldependent increase in central nervous system injury in the fetal sheep. American journal of obstetrics and gynecology. 2009;200(4):437 e1-8. doi:10.1016/j.ajog.2008.12.003. 
24. Hankin JA, Farias SE, Barkley RM, Heidenreich K, Frey LC, Hamazaki K et al. MALDI mass spectrometric imaging of lipids in rat brain injury models. Journal of the American Society for Mass Spectrometry. 2011;22(6):1014-21. doi:10.1007/ s13361-011-0122-z.

25. Jellema RK, Lima Passos V, Zwanenburg A, Ophelders DR, De Munter S, Vanderlocht J et al. Cerebral inflammation and mobilization of the peripheral immune system following global hypoxia-ischemia in preterm sheep. Journal of neuroinflammation. 2013;10:13. doi:10.1186/1742-2094-10-13.

26. Lardenoije R, van den Hove DLA, Vaessen TSJ, Iatrou A, Meuwissen KPV, van Hagen BTJ et al. Epigenetic modifications in mouse cerebellar Purkinje cells: effects of aging, caloric restriction, and overexpression of superoxide dismutase 1 on 5-methylcytosine and 5-hydroxymethylcytosine. Neurobiology of aging. 2015;36(11):3079-89. doi:10.1016/j. neurobiolaging.2015.08.001.

27. Schwartz M, Baruch $K$. The resolution of neuroinflammation in neurodegeneration: leukocyte recruitment via the choroid plexus. The EMBO journal. 2014;33(1):7-22. doi:10.1002/embj.201386609.

28. Chen Y, Ozturk NC, Zhou FC. DNA methylation program in developing hippocampus and its alteration by alcohol. PloS one. 2013;8(3):e60503. doi:10.1371/journal.pone.0060503.

29. Wang Z, Tang B, He Y, Jin P. DNA methylation dynamics in neurogenesis. Epigenomics. 2016;8(3):401-14. doi:10.2217/epi.15.119.

30. Veloso A, Astigarraga E, Barreda-Gomez G, Manuel I, Ferrer I, Giralt MT et al. Anatomical distribution of lipids in human brain cortex by imaging mass spectrometry. Journal of the American Society for Mass Spectrometry. 2011;22(2):329-38. doi:10.1007/s13361-0100024-5.

31. Carter CL, McLeod CW, Bunch J. Imaging of phospholipids in formalin fixed rat brain sections by matrix assisted laser desorption/ionization mass spectrometry. Journal of the American Society for Mass Spectrometry. 2011;22(11):1991-8. doi:10.1007/s13361-011-0227-4.

32. Angerer TB, Dowlatshahi Pour M, Malmberg P, Fletcher JS. Improved molecular imaging in rodent brain with time-of-flight-secondary ion mass spectrometry using gas cluster ion beams and reactive vapor exposure. Analytical chemistry. 2015;87(8):4305-13. doi:10.1021/ ac504774y.

33. Carson MJ, Thrash JC, Walter B. The cellular response in neuroinflammation: The role of leukocytes, microglia and astrocytes in neuronal death and survival. Clinical neuroscience research. 2006;6(5):237-45. doi:10.1016/j.cnr.2006.09.004.

34. Burd I, Brown A, Gonzalez JM, Chai J, Elovitz MA. A mouse model of term chorioamnionitis: unraveling causes of adverse neurological outcomes. Reproductive sciences. 2011;18(9):900-7. doi:10.1177/1933719111398498.

35. Yang Y, Higashimori H, Morel L. Developmental maturation of astrocytes and pathogenesis of neurodevelopmental disorders. Journal of neurodevelopmental disorders. 2013;5(1):22. doi:10.1186/1866-1955-5-22. 
36. Sharma R, Fischer MT, Bauer J, Felts PA, Smith KJ, Misu T et al. Inflammation induced by innate immunity in the central nervous system leads to primary astrocyte dysfunction followed by demyelination. Acta neuropathologica. 2010;120(2):223-36. doi:10.1007/s00401-010-0704-z.

37. Bonestroo HJ, Heijnen CJ, Groenendaal F, van Bel F, Nijboer CH. Development of cerebral gray and white matter injury and cerebral inflammation over time after inflammatory perinatal asphyxia. Developmental neuroscience. 2015;37(1):78-94. doi:10.1159/000368770.

38. Volpe JJ. Neurobiology of periventricular leukomalacia in the premature infant. Pediatric research. 2001;50(5):553-62. doi:10.1203/00006450-200111000-00003.

39. Penn AA, Gressens P, Fleiss B, Back SA, Gallo V. Controversies in preterm brain injury. Neurobiology of disease. 2016;92(Pt A):90-101. doi:10.1016/j.nbd.2015.10.012.

40. Segovia KN, McClure M, Moravec M, Luo NL, Wan Y, Gong X et al. Arrested oligodendrocyte lineage maturation in chronic perinatal white matter injury. Annals of neurology. 2008;63(4):520-30. doi:10.1002/ana.21359.

41. Back SA, Miller SP. Brain injury in premature neonates: A primary cerebral dysmaturation disorder? Annals of neurology. 2014;75(4):469-86. doi:10.1002/ ana.24132.

42. Kamasawa N, Sik A, Morita M, Yasumura T, Davidson KG, Nagy JI et al. Connexin-47 and connexin-32 in gap junctions of oligodendrocyte somata, myelin sheaths, paranodal loops and Schmidt-Lanterman incisures: implications for ionic homeostasis and potassium siphoning. Neuroscience. 2005;136(1):65-86. doi:10.1016/j. neuroscience.2005.08.027.

43. Ficz G, Branco MR, Seisenberger S, Santos F, Krueger F, Hore TA et al. Dynamic regulation of 5-hydroxymethylcytosine in mouse ES cells and during differentiation. Nature. 2011;473(7347):398-402. doi:10.1038/nature10008.

44. Fleiss B, Gressens P. Tertiary mechanisms of brain damage: a new hope for treatment of cerebral palsy? The Lancet Neurology. 2012;11(6):556-66. doi:10.1016/S14744422(12)70058-3.

45. Favrais G, van de Looij Y, Fleiss B, Ramanantsoa N, Bonnin P, Stoltenburg-Didinger $\mathrm{G}$ et al. Systemic inflammation disrupts the developmental program of white matter. Annals of neurology. 2011;70(4):550-65. doi:10.1002/ana.22489.

46. van Tilborg E, Heijnen CJ, Benders MJ, van Bel F, Fleiss B, Gressens P et al. Impaired oligodendrocyte maturation in preterm infants: Potential therapeutic targets. Progress in neurobiology. 2016;136:28-49. doi:10.1016/j.pneurobio.2015.11.002.

47. Jirtle RL, Skinner MK. Environmental epigenomics and disease susceptibility. Nature reviews Genetics. 2007;8(4):253-62. doi:10.1038/nrg2045.

48. Bilbo SD, Schwarz JM. Early-life programming of later-life brain and behavior: a critical role for the immune system. Frontiers in behavioral neuroscience. 2009;3:14. doi:10.3389/neuro.08.014.2009. 
49. Hagberg H, Mallard C, Ferriero DM, Vannucci SJ, Levison SW, Vexler ZS et al. The role of inflammation in perinatal brain injury. Nature reviews Neurology. 2015;11(4):192-208. doi:10.1038/nrneurol.2015.13.

50. Sun M, Song MM, Wei B, Gao Q, Li L, Yao B et al. 5-Hydroxymethylcytosinemediated alteration of transposon activity associated with the exposure to adverse in utero environments in human. Human molecular genetics. 2016. doi:10.1093/hmg/ ddw089.

51. Li S, Papale LA, Kintner DB, Sabat G, Barrett-Wilt GA, Cengiz P et al. Hippocampal increase of $5-\mathrm{hmC}$ in the glucocorticoid receptor gene following acute stress. Behavioural brain research. 2015;286:236-40. doi:10.1016/j.bbr.2015.03.002.

52. Szulwach KE, Li X, Li Y, Song CX, Wu H, Dai Q et al. 5-hmC-mediated epigenetic dynamics during postnatal neurodevelopment and aging. Nature neuroscience. 2011;14(12):1607-16. doi:10.1038/nn.2959.

53. Meyer U. Prenatal poly(i:C) exposure and other developmental immune activation models in rodent systems. Biological psychiatry. 2014;75(4):307-15. doi:10.1016/j. biopsych.2013.07.011.

54. Jin R, Yu S, Song Z, Quillin JW, Deasis DP, Penninger JM et al. Phosphoinositide 3-kinase-gamma expression is upregulated in brain microglia and contributes to ischemia-induced microglial activation in acute experimental stroke. Biochemical and biophysical research communications. 2010;399(3):458-64. doi:10.1016/j.bbrc.2010.07.116.

55. Eklind S, Mallard C, Arvidsson P, Hagberg H. Lipopolysaccharide induces both a primary and a secondary phase of sensitization in the developing rat brain. Pediatric research. 2005;58(1):112-6. doi:10.1203/01.PDR.0000163513.03619.8D.

56. Mallard C, Hagberg H. Inflammation-induced preconditioning in the immature brain. Seminars in fetal \& neonatal medicine. 2007;12(4):280-6. doi:10.1016/j.siny.2007.01.014.

57. Cao M, Cortes M, Moore CS, Leong SY, Durosier LD, Burns P et al. Fetal microglial phenotype in vitro carries memory of prior in vivo exposure to inflammation. Frontiers in cellular neuroscience. 2015;9:294. doi:10.3389/fncel.2015.00294.

58. Gustavsson M, Anderson MF, Mallard C, Hagberg H. Hy poxic preconditioning confers long-term reduction of brain injury and improvement of neurological ability in immature rats. Pediatric research. 2005;57(2):305-9. doi:10.1203/01.PDR.0000151122.58665.70. 


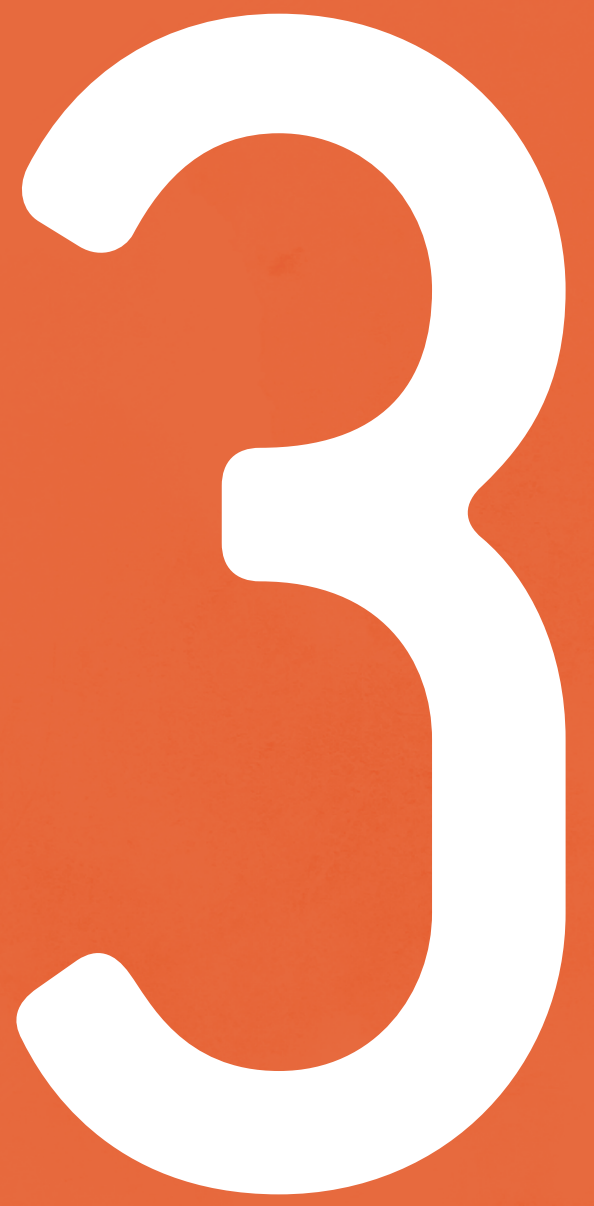

Chorioamnionitis,

neuroinflammation and injury;

timing is key in the preterm

ovine fetus 
Ruth Gussenhoven, Rob J. J.

Westerlaken, Daan R.M.G. Ophelders, Alan H. Jobe, Matthew W. Kemp, Suhas G. Kallapur, Luc J. Zimmermann, Per T. Sangild, Stanislava Pankratova, Pierre Gressens, Boris W. Kramer, Bobbi Fleiss, Tim G.A.M. Wolfs

Journal of Neuroinflammation.

2018;15(1):113 


\section{Abstract}

\section{Background}

Antenatal infection (i.e. chorioamnionitis) is an important risk factor for adverse neurodevelopmental outcomes after preterm birth. Destructive and developmental disturbances of the white matter are hallmarks of preterm brain injury. Understanding the temporal effects of antenatal infection in relation to the onset of neurological injury is crucial for the development of neurotherapeutics for preterm infants. However, these dynamics remain unstudied.

\section{Methods}

Time-mated ewes were intra-amniotically injected with lipopolysaccharide at 5 , 12 , or 24 hours or $2,4,8$, or 15 days before preterm delivery at 125 days gestational age (term $\sim 150$ days). Post mortem analyses for peripheral immune activation, neuroinflammation and white matter/ neuronal injury were performed. Moreover, considering the neuroprotective potential of erythropoietin (EPO) for perinatal brain injury we evaluated (phosphorylated) EPO receptor (pEPOR) expression in the fetal brain following LPS exposure.

\section{Results}

Intra-amniotic exposure to this single bolus of LPS resulted in a biphasic systemic IL-6 and IL-8 response. In the developing brain, intra-amniotic LPS exposure induces a persistent microgliosis (IBA-1 immunoreactivity) but a shorterlived increase in the pro-inflammatory marker COX-2. Cell death (caspase-3 immunoreactivity) was only observed when LPS exposure was greater than 8 days in the white matter, and there was a reduction in the number of (pre) oligodendrocytes (Olig2 and PDGFR $\alpha$ positive cells) within the white matter at 15 days post LPS exposure only. pEPOR expression displayed a striking biphasic regulation following LPS exposure which may help explain contradicting results amongst clinical trials that tested EPO for the prevention of preterm brain injury.

\section{Conclusion}

We provide increased understanding of the spatiotemporal pathophysiological changes in the preterm brain following intra-amniotic inflammation which may aid development of new interventions or implement interventions more effectively to prevent perinatal brain damage. 


\section{Background}

Antenatal infections (i.e. chorioamnionitis) are an important risk factor for preterm birth and a major contributor to neonatal morbidity and mortality $[1,2]$. Intra-amniotic exposure to micro-organisms and subsequent induction of inflammatory mediators in the amniotic cavity can initiate a fetal systemic immune response that is characterized by increased plasma interleukin (IL)-6 and IL-8 concentrations [3], and (persistent) changes in essential immunological organs including the fetal spleen and thymus $[4,5]$. At the crosstalk between fetal peripheral blood and the brain (i.e. blood-brain barrier), this systemic inflammatory response can initiate a detrimental neuroinflammatory response which is primarily mediated by microglia and peripheral immune effector cells $[6,5]$. This cerebral inflammatory response is a risk factor for preterm brain injury and concomitant adverse neurodevelopmental outcomes including cognitive, behavioral and attentional impairments and motor dysfunctions (i.e. cerebral palsy) $[7,5]$.

In a pre-clinical chorioamnionitis model, we showed that short-term (2 days) intra-amniotic exposure to lipopolysaccharide (LPS) resulted in systemic inflammation, overt microgliosis and changes in myelin basic protein (MBP) immunoreactivity (IR) in the fetal ovine brain [8]. However, this systemic and cerebral phenotype was substantially different following longer exposure time (7 days) indicating that time-dependent peripheral and cerebral changes occur following intra-amniotic inflammation. Moreover, we and others have shown that inflammation can modulate a second inflammatory stimulus through either preconditioning or sensitization of the fetal brain $[9,8,10]$. Taken together, this emphasizes that inflammation as pathogenic mediator for brain damage is not a single trigger within a short time frame but more a dynamic process over an extended period of time. Therefore, detailed studies elucidating the timedependent effects of antenatal infection/inflammation in relation to neurological injury and development are crucial to gain insight in the pathophysiological changes in the fetal brain following antenatal stress. Importantly, such temporal insight in the induction of brain injury following antenatal stress is also essential to define the therapeutic window of opportunity for neurotherapeutics. 
One of the most promising treatment options for preterm neonates at high risk for brain injury is erythropoietin (EPO), an important cytokine for brain development [11, 12]. Multiple experimental and clinical studies have demonstrated efficacy of EPO administration to prevent injury to the preterm brain, including severe periventricular leukomalacia, without adverse effects [13-21]. In contrast, other clinical trials do not report improvement in neurodevelopment following EPO treatment [22, 23]. Effects of EPO are mediated by its receptor, which is abundantly present on (pre)oligodendrocytes, astrocytes, microglia and neurons. EPO binding triggers phosphorylation of two monomers, which in turn phosphorylates and activates the signaling kinase Jak-2 facilitating effects including its anti-inflammatory, anti-oxidative and anti-apoptotic properties [24, 25]. In addition, EPO enhances neuro- and oligodendrogenesis, oligodendrocyte maturation and myelin production which are indispensable events in injury repair and normal neurodevelopment [26]. We hypothesize that changes in basal levels of EPO receptor activation in response to inflammation or perinatal stress might explain at least part of the differences in clinical outcomes following EPO treatment.

Considering the clinical need for understanding the time-dependent cerebral changes following intra-amniotic inflammation, we performed a detailed analysis of the temporal dynamics of intra-amniotic LPS-induced systemic and cerebral inflammation and subsequent fetal brain injury. In addition, to optimize EPO treatment in the clinical setting we analyzed the temporal expression of the phosphorylated EPO receptor (pEPOR) in the course of intra-amniotic inflammation. 


\section{Methods}

\section{Study approval}

Animal procedures were performed with approval of the animal ethics committee of the University of Western Australia (Perth, Australia).

\section{Experimental design}

The design of this study was published previously [4]. Briefly, fifty-two timemated ewes with singleton fetuses were randomly allocated in groups of 5-7 animals per group to receive an intra-amniotic injection under ultrasound guidance with an established dose [27] of $10 \mathrm{mg}$ Escherichia coli-derived LPS (O55:B5; Sigma-Aldrich, St. Louis, MO) at 5, 12 or 24 hours (h) or 2, 4, 8 or 15 days (d) before preterm delivery at 125 days of gestation (term $\sim 150$ days) (Figure 1). This paradigm is based on the clinical paradigm, where we know the gestational age of the infant but not the length of exposure to inflammation. As such all our tissues were collected at a known gestation age but inflammation was induced at various times before. The half-life time of LPS in the amniotic fluid is relatively long (1.7 days) and LPS concentrations remain detectable till 15 days after injection [28]. Moreover, intra-amniotic delivery of $0.1 \mathrm{mg}$ LPS, a bolus which in this study is reached at 10 days after injection, still results in an influx of inflammatory cells in the amniotic fluid and fetal lungs [27] indicating that IA delivery of 10mg LPS is a clinical relevant ongoing inflammatory stimulus. Fetuses of either sex were used and previous analysis of the thymus reported no sex specific differences in this model [4]. Control animals received an equivalent volume of $0.9 \%$ saline solution (SAL; controls) at variable gestational ages comparable to LPS injections, ranging from $5 \mathrm{~h}$ to $15 \mathrm{~d}$ before preterm delivery. Within this control group no differences were observed between different lengths of saline exposure for which we have pooled these animals in one control group (SAL). At 125 days of gestation, when ovine brain development is similar to 32-34 weeks of human gestation [29], all fetuses were surgically delivered and immediately euthanized with intravenous pentobarbitone (100 mg/kg). Fetal blood was collected and the brains were removed and immersion fixed in $4 \%$ paraformaldehyde. 


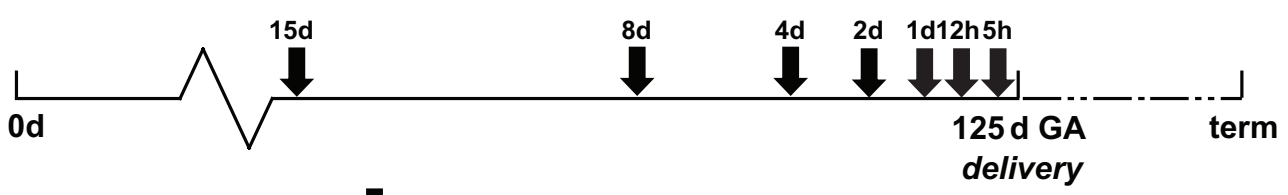

Figure 1. Study design. Pregnant ewes received an intra-amniotic injection with $10 \mathrm{mg}$ Escherichia coli-derived lipopolysaccharide (LPS) at 5, 12 or 24 hours (h) or 2, 4, 8 or 15 days (d) (black arrows) before preterm delivery at 122 days of gestation (term 150 days). Control animals received an intra-amniotic injection with an equivalent volume of $0.9 \%$ saline solution at comparable time points to LPS injections.

\section{Analysis of blood IL-6 and IL-8 concentration}

Levels of the pro-inflammatory cytokines interleukin (IL)-6 and IL-8 were measured in fetal plasma as markers for systemic inflammation using ovine specific sandwich enzyme-linked immunosorbent assays (ELISA) as previously described [8].

In short, a 96-wells plate was coated with a monoclonal mouse-anti IL-6 (Millipore Cat\# MAB1004, working concentration 1:200) or IL-8 (Millipore Cat\# MAB1044, working concentration 1:200) and incubated overnight at $4^{\circ} \mathrm{C}$. The standard curve and serum samples were diluted in PBS $+0.1 \%$ BSA in 1:1 or 1:80 respectively for IL-6 and IL-8. Incubation with the detection antibody rabbit-anti-ovine IL-6 (Millipore Cat\# AB1839, working concentration 1:500) or IL-8 (AB1040, Millipore, working concentration 1:500) was performed for 1 hour, followed by incubation with a HRP-labelled antibody (Jackson ImmunoResearch Labs Cat\# 111-035-045, working concentration 1:500). Next, incubation with 3,3'5,5'-Tetramethylbenzidine (TMB) substrate solution was done for 10 (IL-6) or 2,5 (IL-8) minutes. The reaction was stopped by addition of $\mathrm{H} 2 \mathrm{SO} 4$ and the optical density (OD) was measured at 450nm in a Thermo Electron Type 1500 Multiskan Spectrum Microplate Reader. Concentrations were expressed relative to a standard curve of recombinant ovine IL-6 or IL-8 (ImmunoChemistry Technologies, Bloomington, MN, USA).

\section{Histology and immunohistochemistry}

The cerebral white matter and hippocampus are most commonly affected by intra-amniotic infections at this developmental stage [30]. Therefore, we have chosen to assess inflammatory and structural changes within these regions of interest. After fixation, a predefined region containing the posterior hippocampus/mid-thalamus of the left hemisphere was embedded in paraffin and serial coronal sections $(4 \mu \mathrm{m})$ were cut with a Leica RM2235 microtome. 
Hematoxylin and eosin (H\&E) staining was performed for structural and morphological analysis. Immunohistochemical staining was performed on four slides per staining per animal (every 10th consecutive slide) as previously reported [8]. Inflammatory changes were assessed by the following immunohistochemical markers: cylcooxygenase-2 (COX-2) (1:50, Cayman Chemical; aa570-598), ionized calcium-binding adapter molecule 1 (IBA-1) (1:1000, Wako Pure Chemical Industries, Osaka, Japan) and glial fibrillary acidic protein (GFAP) (1:1000, DAKO Z0334). The presence of neutrophils was assessed by myeloperoxidase (MPO) staining (1:200, DAKO A0398). Markers used to assess alterations in the white matter including oligodendrocyte differentiation were oligodendrocyte transcription factor 2 (Olig2) (1:200, Millipore, 13 AB9610), platelet-derived growth factor receptor alpha (PDGFRa) (1:100, Santa Cruz Biotechnology, sc338), 2',3'-Cyclic-nucleotide 3'-phosphodiesterase (CNPase) (1:1000, Sigma, C5922) and myelin basic protein (MBP) (1:1000, Merck Millipore, MAB386). Neuronal architecture, including cell bodies and dendrites, was determined by microtubule associated protein-2 (MAP-2) (1:500, Sigma, M9942). Apoptotic cell death was measured as cells positive for cleaved caspase-3 (1:1000, cell signaling, \#9661) and the number of mitotic cells were identified by phosphoHistone H3 (pHH3) (1:100, Santa Cruz Biotechnology, sc-101679). The presence of the erythropoietin receptor was assessed by measuring the expression of the (phosphorylated) erythropoietin receptor (EPOR and pEPOR) (1:200, Santa Cruz, SC-365662 and SC-20236).

Deparaffinization and rehydration was performed by incubation in xylol and decreasing alcohol concentrations. Endogenous peroxidase activity was quenched via incubation with $0.3 \% \mathrm{H}_{2} \mathrm{O}_{2}$ for 10 minutes. Antigen retrieval involved boiling tissues in citrate buffer $(\mathrm{pH}$ 6.0) for 10 minutes or for $\mathrm{pEPOR}$ proteinase $\mathrm{K}$ at $37^{\circ} \mathrm{C}$ for 5 minutes. Nonspecific binding was prevented by incubation with 5\% (IBA-1, GFAP, MAP2, pHH3) or 10\% (MPO, CNPase) normal goat serum, 5\% bovine serum albumin (COX-2, MBP, Olig2, EPOR) (Invitrogen Thermofisher Scientific) or 10\% nonfat dry milk (pEPOR; Elk, Campina bv., Eindhoven, The Netherlands) for 1 hour. Tissues were incubated with the primary antibody overnight at $4^{\circ} \mathrm{C}$, followed by incubation with the species specific secondary antibody at 1:200 (DAKO) for 1 hour at room temperature. The antibody specific signal was enhanced with a Vectastain $A B C$ peroxidase Elite kit (Vector Laboratories Inc, Burlingame, CA) for 1 hour and 3,3'-diaminobenzide (COX-2, IBA-1, GFAP, MPO, PDGFRa, CNPase, MBP, MAP-2, pHH3, EPOR) or nickel chloride 3,3'-diaminobenzide (Olig2, cleaved caspase-3, pEPOR) for 2-10 minutes. Nuclei were stained with Mayer's hematoxylin. 
Qwin Pro v3.4.0. software (Leica Microsystems, Wetzlar, Germany). Area fractions and integrated densities were calculated for IBA-1, GFAP, COX-2, MBP, MAP-2 and pEPOR. MPO, Olig2, PDGFRa, CNPase, pHH3 and cleaved caspase-3 positive cells were counted and expressed as total cell count per square millimeter (cells/mm2). In addition, $\mathrm{MPO}+$ cells were also counted in the choroid plexus. Values per region of interest were averaged.

\section{Statistical analysis}

All values are shown as mean with 95\% confidence interval (CI) or standard deviations (SD). Comparison between different experimental groups was performed with analysis of variance (ANOVA) or with a random intercept mixed model in case of repeated measurements per animal (e.g., different sections per brain) with Bonferroni correction for multiple comparisons. We applied log-transformation to obtain normal distributed data when data or variables were positively skewed before statistical testing. Statistical analysis was performed with IBM SPSS Statistics Version 22.0 (IBM Corp., Armonk, NY, USA; SPSS). Statistical significance was accepted at $p<0.05$. Considering the relatively low number of animals per group exact $p$-values are provided and $0.05<\mathrm{p}<0.1$ is considered a trend.

\section{Qualitative and quantitative analysis}

An independent neuropathologist and two independent researchers who were blinded for the experimental conditions performed qualitative and quantitative analysis of the tissues. Analysis were performed using a light microscope (Leica DM2000) equipped with Leica QWin Pro version 3.4.0 software (Leica Microsystems, Mannheim, Germany). H\&E stained sections were scored for gliosis, hemorrhages and structural damage like cyst formation. Regions of interest of the white matter and hippocampus were defined as previously described [31]. In addition, grey matter alterations in the cerebral cortex were assessed within the same section. Three to five adjacent images were taken per region of interest at 100x magnification and analyses were performed using Leica 


\section{Results}

\section{Animal characteristics}

At birth, no differences in weight were found between experimental groups. Fetal blood $\mathrm{pH}$ and hemoglobin levels did not differ following intra-amniotic LPS exposure. No sex differences in susceptibility were observed in either readout including animal characteristics and all following readouts regarding systemic cytokine levels and immunohistochemical analysis.

\section{Intra-amniotic LPS exposure results in a biphasic fetal systemic inflammatory response}

Intra-amniotic exposure to LPS results in an acute increase of fetal systemic IL-6 concentrations at 5, 12 and $24 \mathrm{~h}$ after LPS exposure compared to control levels (SAL vs 5h LPS $\mathrm{p}=0.0157$; SAL vs $12 \mathrm{~h}$ LPS $\mathrm{p}=0.0011$; SAL vs $24 \mathrm{~h}$ LPS $\mathrm{p}=0.0035$ ) (Figure 2A). Subsequently, at 4 and 8 days after LPS exposure systemic IL-8 concentrations are increased compared to controls (SAL vs. 4d LPS $p=0.0147$; SAL vs. 8d LPS $\mathrm{p}=0.0502$ ) (Figure 2B).

A

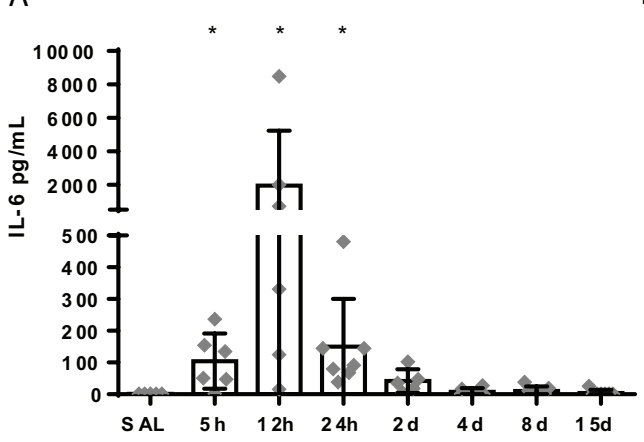

B

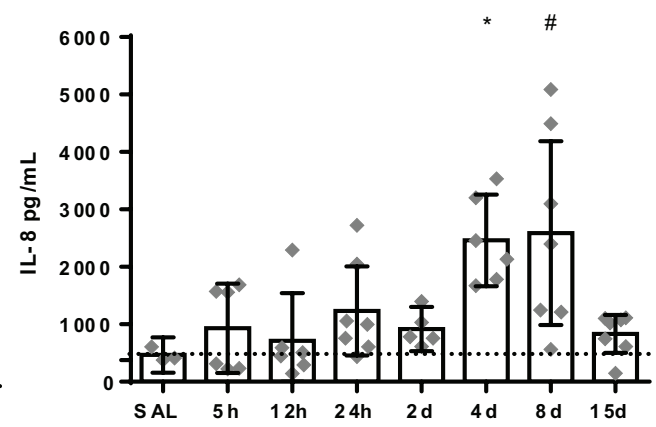

Figure 2. Circulatory interleukin (IL)-6 and IL-8 concentrations illustrate a biphasic response following intra-amniotic LPS exposure. Undetectable values were assigned an arbitrary value of $1 \mathrm{pg} / \mathrm{mL}$ in order to perform statistical analysis. Statistical analysis was done with ANOVA and values are expressed as mean \pm 95\% CI. * indicated $\mathrm{p}<0.05$ versus control group, \# indicated $0.05<\mathrm{p}<0.1$ versus control. 


\section{Cerebral inflammation in the fetal white matter, hippocampus and cortex following intra-amniotic LPS exposure}

A systemic fetal inflammatory response is postulated to initiate cerebral inflammation leading to subsequent injury [32-34]. Therefore, we initially measured markers of changes in neuroinflammatory processes (IBA-1, COX-2, GFAP). We found that the systemic inflammatory response following intra-amniotic LPS exposure is followed by cerebral inflammatory changes as indicated by an increase in IBA-1 IR in the white matter at 12 hours, 2, 4 and 8 days following LPS exposure compared to controls (SAL vs. 12h LPS $\mathrm{p}=0.012$; SAL vs. $2 \mathrm{~d}$ LPS $\mathrm{p}=0.006$; SAL vs. $4 \mathrm{~d}$ LPS $\mathrm{p}=0.005$; SAL vs. $8 \mathrm{~d}$ LPS $\mathrm{p}=0.088$ ) (Figure 3A and 3B). In the hippocampus, inflammation is detected by an acute increase in COX-2 IR at 5, 12 and 24 hours post LPS exposure (SAL vs. 5h LPS $\mathrm{p}=0.055$; SAL vs. $12 \mathrm{~h}$ LPS $\mathrm{p}=0.016$; SAL vs. 24h LPS $\mathrm{p}=0.096$ ) (Figure 3C and 3D) and an increase in IBA-1 IR at 15 days after LPS exposure compared to controls (SAL vs. 15d LPS $p=0.073$ ). There were no changes of GFAP IR after LPS exposure at any of the time points. As outlined in Table 1 the number of MPO+ cells is increased in the white matter at $15 \mathrm{~d}$ post LPS exposure (SAL vs. $15 \mathrm{dLPS} p=0,027$ ). In the choroid plexus, no significant differences of $\mathrm{MPO}+$ cells were found between groups (Table 1).

\begin{tabular}{lllllllll}
\hline $\begin{array}{l}\text { MPO+ } \\
\text { cells } / \text { mm }^{2}\end{array}$ & SAL & 5hLPS & $\mathbf{1 2 h L P S}$ & $\mathbf{2 4 h L P S}$ & 2dLPS & 4dLPS & 8dLPS & 15dLPS \\
Choroid & $2,44 \pm$ & $4,34 \pm$ & $3,19 \pm$ & $1,80 \pm$ & $3,88 \pm$ & $6,25 \pm$ & $4,07 \pm$ & $3,51 \pm$ \\
plexus & 3,22 & 2,86 & 3,44 & 1,45 & 2,54 & 4,25 & 2,67 & 4,38 \\
White & $0,47 \pm$ & $3,83 \pm$ & $1,14 \pm$ & $1,95 \pm$ & $1,88 \pm$ & $2,05 \pm$ & $2,67 \pm$ & $4,52 \pm$ \\
matter & 0,30 & 3,82 & 0,83 & 2,46 & 2,70 & 1,84 & 2,50 & $4,22^{*}$ \\
\hline
\end{tabular}

Table 1. MPO-positive cells in the choroid plexus and white matter. Mean values \pm standard deviations are represented. ${ }^{*} \rho<0,05$. 
SAL
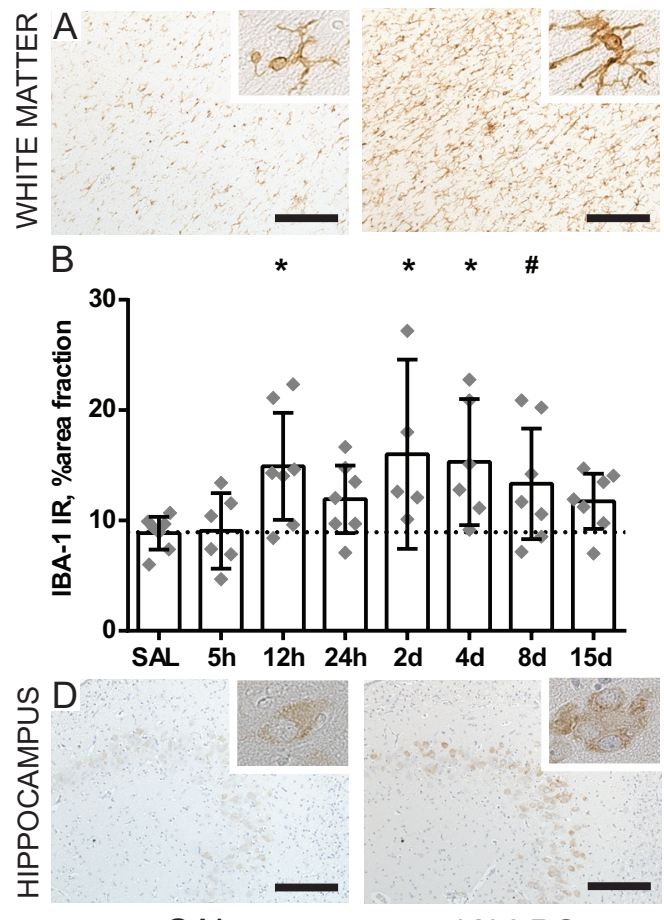

SAL

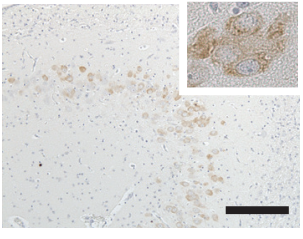

12hLPS
8dLPS
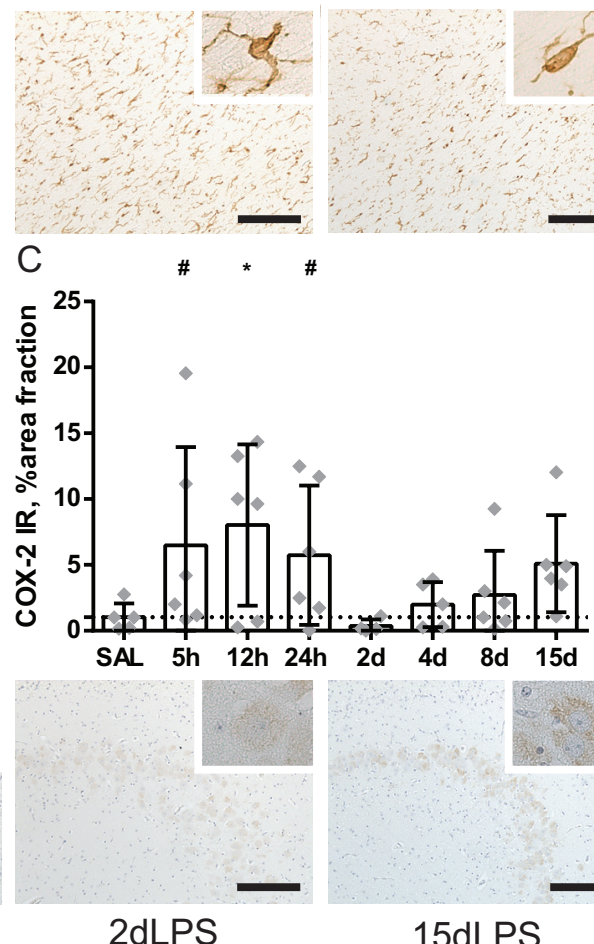

15dLPS
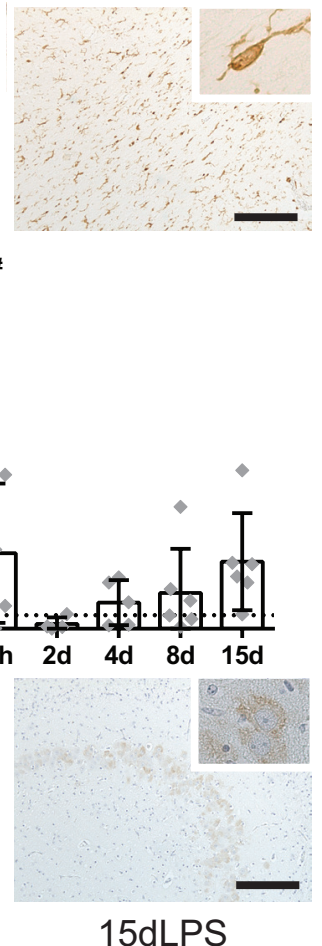

Figure 3 Intra-amniotic exposure to LPS induces an acute, transient cerebral inflammatory response in the preterm white matter and hippocampus. An increase of the area fraction of IBA-1 immunoreactivity (IR) was observed in the white matter at 12 hours, 2, 4 and 8 days following LPS exposure compared to controls (SAL vs. 12h LPS $\mathrm{p}=0.012$; SAL vs. $2 \mathrm{~d}$ LPS $\mathrm{p}=0.006$; SAL vs. $4 \mathrm{~d}$ LPS $\mathrm{p}$ $=0.005$; SAL vs. $8 \mathrm{~d}$ LPS $\mathrm{p}=0.088$ ) (A \& B). In the hippocampus, an increase of the area fraction of COX-2 IR was found at 5, 12 and 24 hours following LPS exposure (SAL vs. 5h LPS p = 0.055; SAL vs. 12h LPS $\mathrm{p}=0.016$; SAL vs. 24h LPS $\mathrm{p}=0.096)(\mathrm{C} \& \mathrm{D})$. Representative histological figures of the IBA-1 positive microglia in animals exposed to intra-amniotic saline (SAL), 2d, 8d and 15d of LPS are shown in (A). Representative histological figures of COX-2 positive neurons in the hippocampus of animals exposed to saline (SAL), 12h, 2d and 15d LPS are depicted in (D). IBA-1 IR and COX-2 IR are depicted as mean \% area fraction $\pm 95 \%$ CI. * indicated $\mathrm{p}<0.05$ versus control (SAL), \# indicated $0.05<\mathrm{p}<0.1$ versus control (SAL). Images taken at 100x magnification (insert at 400x magnification), scale bar $=200 \mu \mathrm{m}$. 

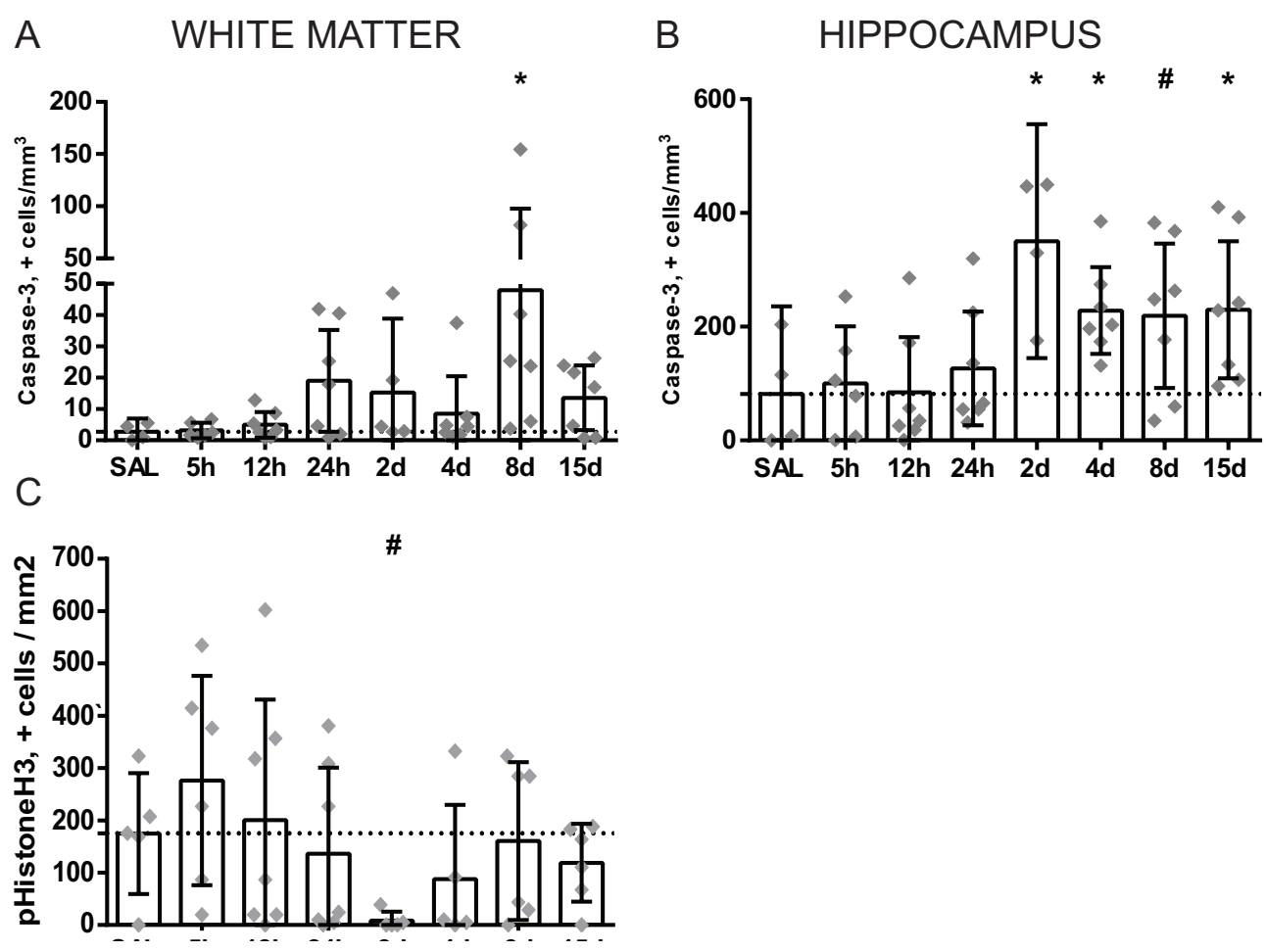

Figure 4. Intra-amniotic exposure to LPS results in a decrease in mitotic cells and relatively late onset of cell death in the preterm white matter and hippocampus. A significant increase of caspase-3 positive cells is observed at 8 days following LPS exposure in the white matter compared to controls (SAL vs $8 \mathrm{~d}$ LPS $\mathrm{p}=0.004$ ) (A). In the hippocampus, at 2, 4, 8 and 15 days following LPS exposure an increase in caspase- 3 positive cells was found compared to controls (SAL vs 2d LPS $\mathrm{p}=0.002$; SAL vs $4 \mathrm{~d}$ LPS $\mathrm{p}=0.030$; SAL vs $8 \mathrm{~d}$ LPS $\mathrm{p}=0.058$; SAL vs $15 \mathrm{~d}$ LPS $\mathrm{p}=0.042)(\mathrm{B})$. At $2 \mathrm{~d}$ following LPS exposure a decrease in $\mathrm{pHH} 3+$ cells was found compared to controls (SAL vs. 2dLPS p =0.100) (C). Caspase-3 and pHH3 are expressed as positive cells / $\mathrm{mm} 2$ and represented in the graphs as mean $\pm 95 \%$ CI. * indicated $\mathrm{p}<0.05$ versus control (SAL), \# indicated $0.05<\mathrm{p}<0.1$ versus control (SAL).

\section{Cell death and proliferation in the fetal white matter, hippocampus and cortex following intra-amniotic LPS exposure}

To assess whether cerebral inflammation is followed by tissue injury we measured the number of caspase- 3 positive cells in the cerebral white matter, hippocampus and cortex as a marker of apoptotic cell death as this is an important prognostic factor for neurological outcomes [35]. Exposure to LPS results in an increase in cleaved caspase-3 positive cells in the white matter at 8 days following LPS exposure (SAL vs $8 d$ LPS $p=0.004$ ), in the hippocampus at 2, 4, 8 and 15 days following LPS exposure (SAL vs $2 \mathrm{~d}$ LPS $\mathrm{p}=0.002$; SAL vs $4 \mathrm{~d}$ LPS $\mathrm{p}=0.030$; SAL vs $8 \mathrm{~d}$ LPS $\mathrm{p}=0.058$; 
SAL vs $15 d$ LPS $p=0.042)$ and in the cortex at 8 days following LPS exposure (SAL vs $8 \mathrm{~d}$ LPS $\mathrm{p}=0.041$ ) compared to controls (Figure $4 \mathrm{~A}$ and $4 \mathrm{~B})$. No evidence of structural changes such as intraventricular hemorrhages and cystic lesions in all experimental groups was found. To assess the proliferation state of the brain, $\mathrm{pHH} 3$ + cells were counted. At 2 $\mathrm{d}$ following LPS exposure a trend towards significant decrease in $\mathrm{pHH} 3+$ cells was found (SAL vs. 2dLPS p $=0.100$ ) (Figure 4C).

\section{Distinct time dependent changes in numbers of oligodendrocyte lineage cells following LPS exposure}

The typical histopathological substrate of brain injury in premature infants consists of injury of the developing oligodendrocyte (OL), a cell type that is abundantly present within the brain during weeks 23-32 of gestation (when preterm birth often occurs) and is prone to inflammatory insults [36]. Therefore, we have assessed oligodendrocyte development following intraamniotic LPS exposure by studying the following oligodendrocyte lineage markers: Olig-2 as a pan-oligodendrocyte lineage marker, PDGFRa as a pre-oligodendrocyte marker (for both OL progenitor and pre-OLs), CNPase as an early oligodendrocyte differentiation marker and MBP for mature oligodendrocytes and myelin. At $15 \mathrm{~d}$ after LPS exposure, a significant decrease in Olig2+ cell number was found compared to controls (SAL vs. $15 \mathrm{dLPS} \mathrm{p}=0.050$ ) (Figure 5A and 5C). At this time point, the PDGFRa+ progenitor and precursor oligodendrocyte populations tended to decrease compared to controls (SAL vs. 15dLPS $\mathrm{p}=0.070$ ) (Figure 5A and 5D). No significant changes of CNPase+ cells and MBP IR were found following LPS exposure compared to controls at all studied time points (Figure 5A, $5 \mathrm{E}$ and $5 \mathrm{~F})$.

\section{Intra-amniotic exposure to LPS resulted in altered dendritic development in grey matter regions of the fetal brain}

Besides alterations in white matter development and oligodendrocyte loss, developmental disturbances of the grey matter are an increasingly important feature of perinatal brain injury [37-39]. For the assessment of dendritic maturation we have studied MAP-2 IR as an established marker for neuronal development [40] in the hippocampus and cerebral cortex. As illustrated in figure 5, intra-amniotic exposure to LPS results in a significant or trend to increase in the MAP-2 IR in the hippocampus at all time points except at 2d LPS (SAL vs 5h LPS $p=0.007$; SAL vs $12 \mathrm{~h}$ LPS $\mathrm{p}=0.073$; SAL vs $24 \mathrm{~h}$ LPS $\mathrm{p}=0.000$; SAL vs $4 \mathrm{~d}$ LPS $\mathrm{p}=0.034$; SAL vs $8 \mathrm{~d}$ LPS $\mathrm{p}=0.000$; 


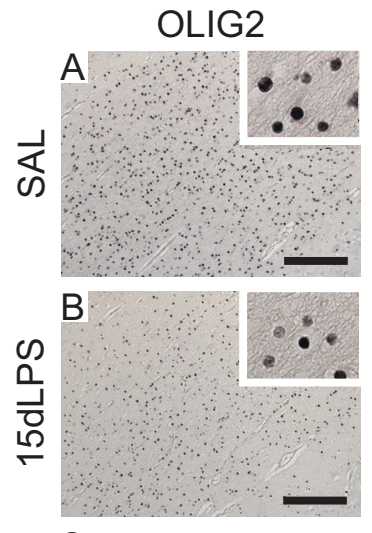

C
PDGFRa

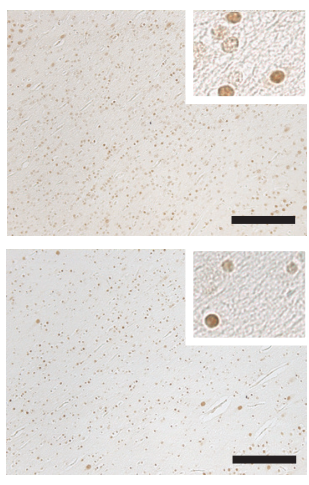

$\mathrm{D}$
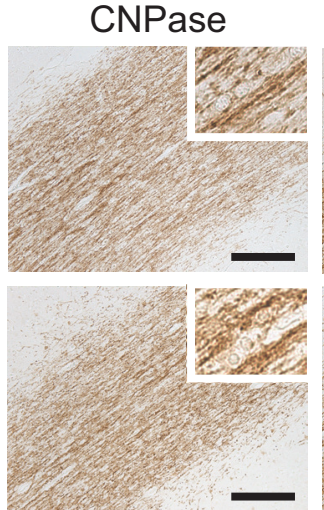

D

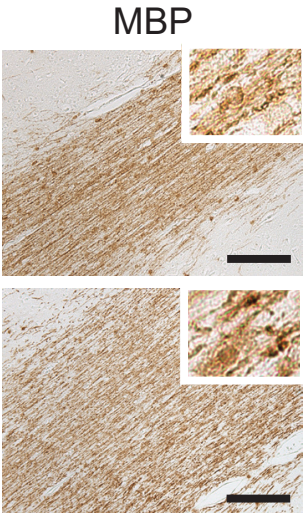

\#
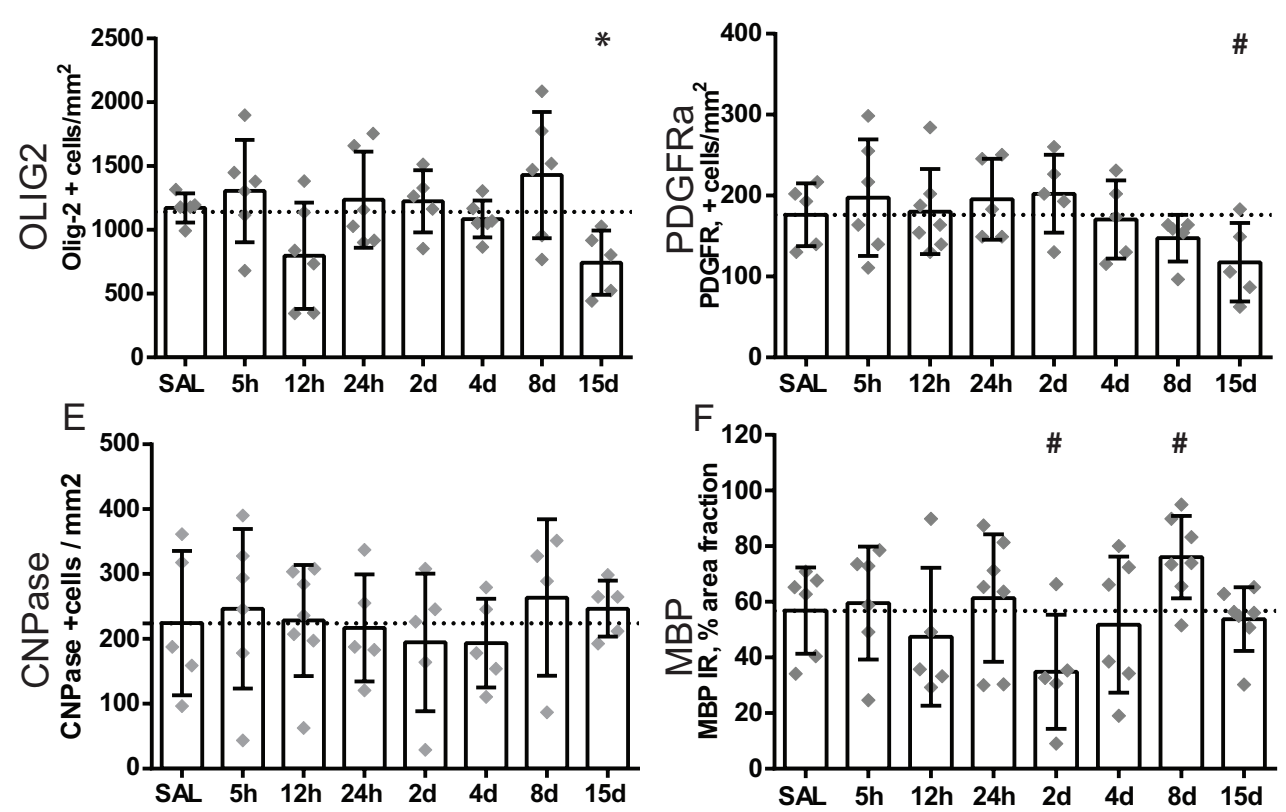

Figure 5. Intra-amniotic exposure to LPS induces distinct time dependent changes in oligodendrocyte lineage cells. A significant decrease of Olig2 positive cells was observed in animals after 15d of LPS exposure compared to controls (SAL vs. 15dLPS $\mathrm{p}=0.050)(\mathrm{C})$. At the same time point a decrease in PDGFRa positive cells was found compared to controls (SAL vs. 15dLPS $p=0.070$ ) (D). No significant changes of CNPase+ cells were found following LPS exposure compared to controls (E). Area fractions $(\%)$ of MBP immunoreactivity (IR) showed a decrease at $2 \mathrm{~d}$ and an increase at $8 \mathrm{~d}$ following LPS exposure compared to controls (SAL vs. 2dLPS, $\mathrm{p}=0.070$; SAL vs. 8dLPS $\mathrm{p}=0.083$ ) (F). Representative histological figures of Olig2, PDGFRa, CNPase positive cells and MBP IR in animals exposed to intra-amniotic saline (SAL) and 15d of LPS are shown in (A) and (B) respectively. Images taken at 100x magnification (insert at 400x magnification), scale bar $=200 \mu \mathrm{m}$. * indicated $\mathrm{p}<0.05$ versus control (SAL), \# indicated $0.05<\mathrm{p}<0.1$ versus control (SAL). 
SAL vs 15d LPS $\mathrm{p}=0.057$ ) (Figure 6A and 6B). At 2 days following LPS exposure MAP-2 IR is comparable to controls (SAL vs 2d LPS p $=0.825)$. In the cerebral cortex only at 24 hours after LPS exposure an increase in MAP-2 IR was found (SAL vs $24 \mathrm{~h}$ LPS $\mathrm{p}=0.036$ ) (Figure 6A and $6 \mathrm{C})$ and all other time points were comparable to control.

\section{Expression of the phosphorylated erythropoietin receptor decreases 2 days following LPS exposure}

There is a single EPO receptor and activation of this receptor leads to receptor phosphorylation and as such phosphorylation is a useful surrogate for EPO-induced downstream pathway activation. Analysis of the IR for total EPOR revealed no change from baseline levels at any of time points of LPS exposure. Interestingly however we observed that $\mathrm{pEPOR}$ had a distinct time-dependent switch from over-expression to under-expression. Specifically, following $5 \mathrm{~h}$ of LPS exposure pEPOR IR was significantly increased in the white matter (SAL vs $5 \mathrm{~h} \mathrm{p}=0.010$ ) (Figure 7A and 7C) and tended to increase in the cortex (SAL vs $5 \mathrm{~h} p=0.100$ ) (Figure 7E) compared to controls. However, at 2 days after LPS exposure there is a strong decrease in pEPOR IR within all brain regions compared to controls; significant in the white matter (SAL vs $2 \mathrm{~d}$ LPS $\mathrm{p}=0.030$ ), and trending in the hippocampus (SAL vs $2 \mathrm{~d}$ LPS $\mathrm{p}=0.088$ ) and cortex (SAL vs $2 \mathrm{~d}$ LPS $\mathrm{p}$ $=0.010$ ) (Figure 7C-7E). At 4 and 8 days following LPS exposure pEPOR expression is still decreased compared to controls; trending in the white matter (SAL vs $4 \mathrm{~d}$ LPS $\mathrm{p}=0.100$ ), and significant in the hippocampus (SAL vs $4 \mathrm{~d}$ LPS 0.045; SAL vs $8 \mathrm{~d}$ LPS $\mathrm{p}=0.014$ ) and cortex (SAL vs $4 \mathrm{~d}$ LPS 0.020; SAL vs $8 \mathrm{~d}$ LPS $\mathrm{p}=0.030$ ). When the fetus had been exposed to $15 \mathrm{~d}$ of LPS there was no drop in $\mathrm{pEPOR} I R$. 


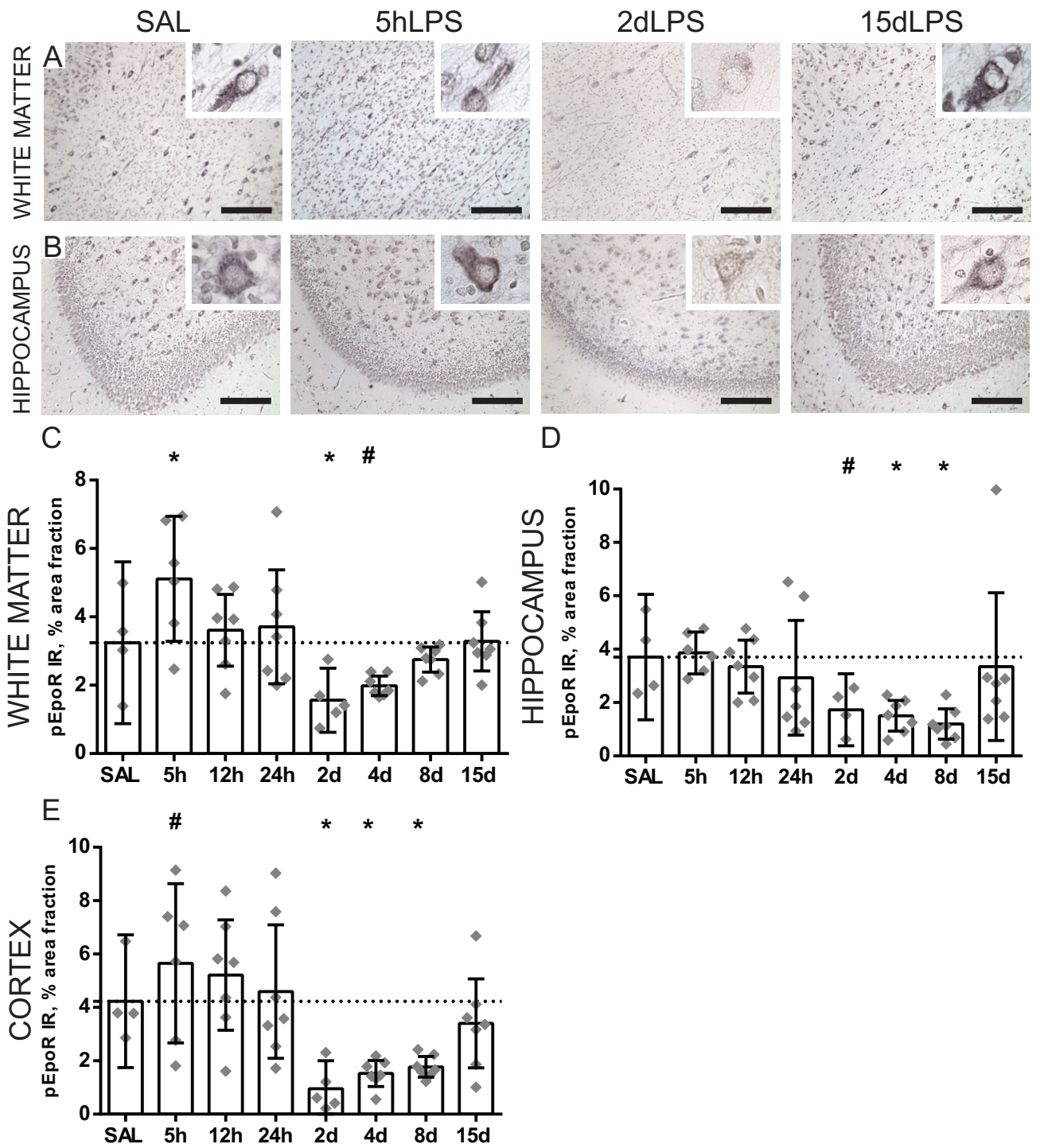

Figure 7. Expression of the phosphorylated erythropoietin receptor decreases 2 days following LPS exposure. An acute increase of the area fraction (\%) of pEPOR immunoreactivity (IR) was observed at $5 \mathrm{~h}$ after LPS exposure in the white matter (SAL vs $5 \mathrm{~h} \mathrm{p}=0.010$ ) and cortex (SAL vs $5 \mathrm{~h} \mathrm{p}=0.100$ ) compared to controls (C \& E). At 2 days after LPS exposure there is a significant decrease in pEPOR IR within all brain regions compared to controls; white matter (SAL vs 2d LPS p $=0.030)(C)$, hippocampus (SAL vs $2 \mathrm{~d}$ LPS $\mathrm{p}=0.088)(\mathrm{D})$ and cortex (SAL vs $2 \mathrm{~d}$ LPS $\mathrm{p}=0.010)(\mathrm{E})$. At 4 and 8 days following LPS exposure pEPOR expression is still decreased compared to controls in the white matter (SAL vs 4d LPS $\mathrm{p}=0.100$ ), hippocampus (SAL vs 4d LPS 0.045; SAL vs 8d LPS $\mathrm{p}=0.014$ ) and cortex (SAL vs 4d LPS 0.020.; SAL vs $8 \mathrm{~d}$ LPS $\mathrm{p}=0.030$ ). When the fetus had been exposed to $15 \mathrm{~d}$ of LPS there was no decrease in $\mathrm{pEPOR}$ IR (C-E). Representative histological figures of the pEPOR in the white matter (A) and hippocampus (B) are depicted in control animals (SAL) and animals exposed to LPS for 5h, 2d and 15d. Images taken at 100x magnification (insert at 400x magnification), scale bar $=200 \mu \mathrm{m}$. * indicated $\mathrm{p}<0.05$ versus controls (SAL), \# indicated $0.05<\mathrm{p}<0.1$ versus controls (SAL). 

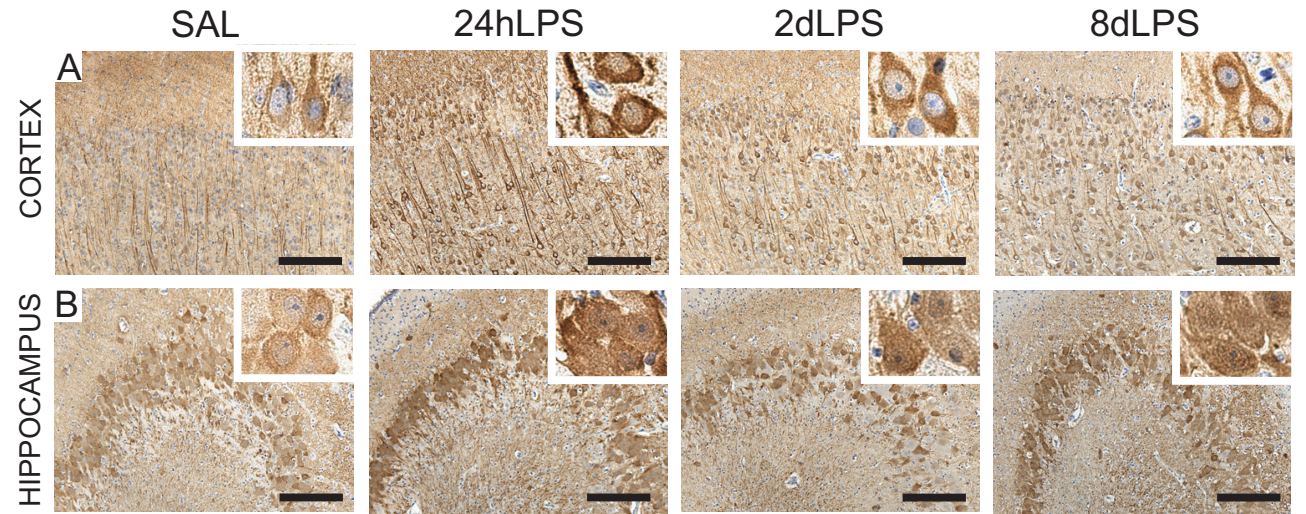

C
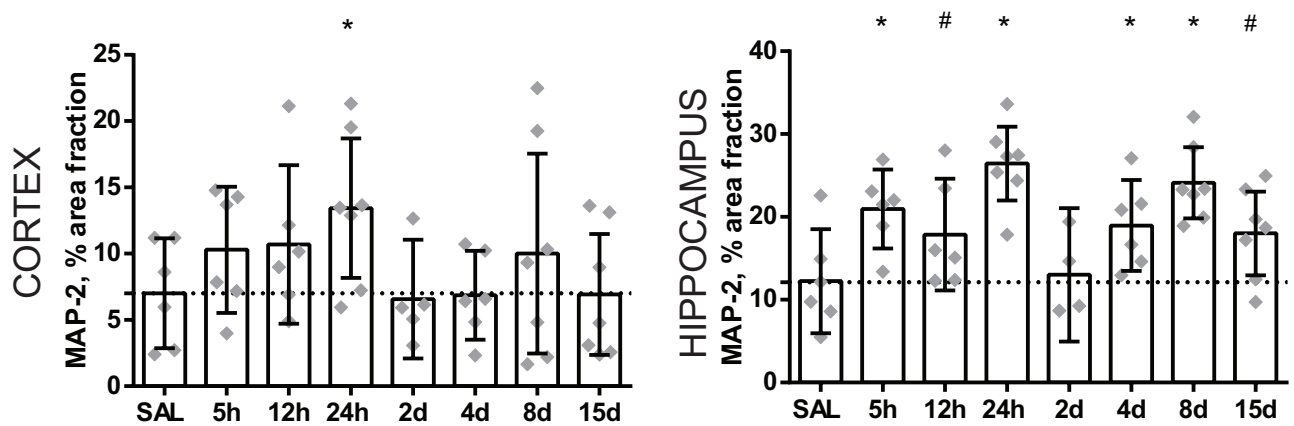

Figure 6. Intra-amniotic exposure to LPS results in altered dendritic development in the grey matter of the fetal brain. A significant increase of the area fraction (\%) of MAP-2 immunoreactivity (IR) was found in the cerebral cortex at $24 \mathrm{~h}$ after LPS exposure compared to controls (SAL vs $24 \mathrm{~h}$ LPS $\mathrm{p}=0.036$ ) (A \& C). In the hippocampus, an increase of area fraction (\%) of MAP-2 IR was observed at 5, 12 and 24 hours and at 4, 8 and 15 days after LPS exposure compared to controls (SAL vs 5h LPS $\mathrm{p}=0.007$; SAL vs $12 \mathrm{~h}$ LPS $\mathrm{p}=0.073$; SAL vs $24 \mathrm{~h}$ LPS $\mathrm{p}=0.000$; SAL vs $4 \mathrm{~d}$ LPS $\mathrm{p}=0.034$; SAL vs $8 \mathrm{~d}$ LPS $\mathrm{p}=0.000$; SAL vs $15 d$ LPS $p=0.057)(B \& D)$. Representative histological figures of MAP-2 in the cerebral cortex $(A)$ and hippocampus (B) are depicted in control animals (SAL) and animals exposed to LPS for $24 \mathrm{~h}, 2 \mathrm{~d}$ and $8 \mathrm{~d}$. Images taken at 100x magnification (insert at 400x magnification), scale bar $=200 \mu \mathrm{m}$. * indicated $\mathrm{p}<0.05$ versus control (SAL), \# indicated $0.05<\mathrm{p}<0.1$ versus control (SAL). 


\section{Discussion}

The main findings of this study are that intra-amniotic exposure to LPS results in 1) an acute onset and biphasic fetal systemic inflammatory response; 2 ) persisting microgliosis with a limited pro-inflammatory phase; 3) cell death and (pre)oligodendrocytes loss only following long exposure to inflammation $(8 \mathrm{~d}+$ ) but 4$)$ a striking regional sensitivity to changes in neuronal architecture following short (hours) and long (days) LPS exposure and 5) biphasic regulation of pEPOR expression.

We demonstrate that fetuses, intra-amniotically exposed to LPS, develop biphasic opposing peaks in the systemic inflammatory response, illustrated by changes in IL- 6 and IL-8 concentrations. This data agree with previous observations from this model on increased circulatory monocytes and neutrophils and subsequent lymphocytopenia [4] and agrees with previous clinical data showing increased levels of these cytokines, associated with adverse neurological outcomes [41, 9]. Furthermore, the systemic response to LPS is consistently associated with the production of a vast milieu of inflammatory cytokines including IL-6 [42]. These signal across the bloodbrain barrier trigger a neuroinflammatory response including activation of microglia and astrocytes [6,5]. Moreover, IL-8 is known as potent chemotactic and neutrophil-activating factor, and increased serum IL-8 levels are reported in newborns with MRI-defined cerebral abnormalities and abnormal neurodevelopmental outcomes [43, 44, 9, 45, 46]. In line, we observed an increased number of $\mathrm{MPO}+$ cells within the cerebral white matter at $15 \mathrm{~d}$ after LPS exposure.

Our study also provides meaningful information about the cerebral inflammatory response initiated by intra-amniotic LPS exposure. We observed a rapid increase in the inflammatory mediator COX-2 and microglial marker IBA-1 in the preterm brain. COX-2 activity results in prostaglandin-E2 production which has wide ranging inflammatory actions on the brain [47], but is typically associated with a pro-inflammatory state in microglia [48] and also reported in astrocytes [49]. Weaver-Mikaere 
et al. demonstrated in a fetal ovine derived mixed glial culture that COX-2 activation is the most important mechanism leading to inflammation-mediated white matter injury [50]. Blocking the COX-2 pathway has recently been shown to prevent hypomyelination and behavioral impairment in mice with neonatal white matter injury [49]. In this model, it has also been reported that there is also no change in GFAP-IR across the groups, but additional analysis revealed that despite this, astrocytes together with microglia were still an important source of oligodendrocyte-injurious COX-2 [49]. An additional injurious mechanism of increased COX-2 is that its primary product prostaglandinE2 stimulates glutamate release from astrocytes [51], which is suggested to be essential in the pathophysiological mechanism underlying neonatal encephalopathy [52]. Additionally, the dynamic temporal microglial response in our inflammatory model, indicated by increased IBA-1IR, is consistent with distinct phases of cerebral inflammation in response to other injurious factors in perinatal brain injury, including a hypoxic-ischemic or excitotoxic insult [9]. Microglial activation is proposed to be essential in cerebral injury and dysmaturation associated with exposure to maternal fetal infection/ inflammation [53]. Of interest for the application of neurotherapeutics is that microglia activation based on the simple proxy of IBA-1IR was maintained for at least 8 days following LPS exposure, but increased COX2 as surrogate for a pro-inflammatory state was only elevated for one day. Further studies to determine a systemic surrogate of microglial activation state might re-invigorate the utility of previously discarded immunomodulatory neurotherapeutics if we understood when immunosuppression would be beneficial.

Considerable debate has raged in the field of preterm neuropathology regarding the role for cell death in encephalopathy of prematurity [54-57]. Experimental data has shown that microglia activation results in pro-inflammatory cytokine release which in turn can lead to apoptosis. However, experimental data has also demonstrated that moderate activation of microglia leads only to the maturation arrest of oligodendrocytes and not cell death [58]. Our study supports a coherent integration of clinical and experimental data as it shows that with a clinically-relevant exposure paradigm the duration of exposure is essential; oligodendrocyte death only occurs after 8 days of LPS exposure. Although outside the scope of this study, research into additional variables including pathogen type and maternal/fetal health and genetics also undoubtedly play a role understanding cohort specific observations on neuropathology. We also 
wish to highlight that the significant loss of (pre)oligodendrocytes was not accompanied by myelin loss in our study. It has been previously postulated that the type of robust reduction in myelin protein levels that we would be able to measure with our analysis technique only occurs later in the course of brain injury due to normal kinetics of myelin production [59]. Furthermore, in an inflammation-induced oligodendrocyte injury model, early analysis of myelin proteins [58] and genes [60] has indeed failed to observe reductions despite later robust hypomyelination and behavioral deficits. Detailed analysis of myelin structure could be considered to investigate early markers of myelin injury in future studies.

Together with disturbed white matter development, alterations in grey matter development are implicated in long-term neurological sequelae following intra-amniotic infections and preterm birth [39, 37, 38]. During normal grey matter development, a decrease in MAP-2IR occurs which is indicative for refining of dendritic branching and spines [40]. Regulation of spine development is part of the basic homeostatic functions of microglia, which also include supporting immature cortical neuronal survival and stimulating myelination [61]. As such, increase in MAP-2 IR that we found in the acute phase following LPS exposure suggests a delay in cortical development, a phenomenon that has also been suggested from analysis of preterm infants using MRI [62]. Moreover, in humans suffering from schizophrenia, increased dendritic arborisation was found in the hippocampus compared to healthy humans when stained for MAP-2 [63]. Concerning the specific mechanistic link between increased inflammation and increased MAP-2, it is noteworthy that COX-2 overexpressing neuronallike cells showed significantly increased neurite outgrowth and branching, which was partially reverted by COX inhibitor [64].

Despite multiple studies identifying EPO as very promising neuroprotective candidate in newborn infants [65, 66, 14, 67, 13, 17,68-70], other studies did not find therapeutic effects on neurodevelopmental outcomes [71, 72, 23]. These inconsistencies might be explained by heterogeneity between study cohorts including, as outlined in this study for the first time, differences in EPO signaling in response to inflammation. Interestingly, after an initial increase in the receptor phosphorylation levels, a distinct and long lasting decrease of the pEPOR occurred. Activation of the EPOR phosphorylates and activates the kinase Jak-2 resulting in initiation of a complex antiapoptotic signaling cascade $[73,25]$. Accordingly, we are unsurprised that a decrease in the level of pEPOR is accompanied by an increase in the number 
of apoptotic cells (caspase-3+) within the same region in the fetal brain. This suggests that the reduced activation of the EPO receptor in our study results in increased apoptosis in the preterm brain and in experimental paradigms this relationship between EPOR and cell death has been demonstrated [25]. In regards to the total expression of EPOR, others have also shown that this remains stable or increases [74], and this elevation occurs within hours in response to pro-inflammatory cytokines like TNFa [75], whereas the level of its parent ligand, EPO, is downregulated under pro-inflammatory conditions [76]. Thus, understanding the inflammationinduced dysregulation of EPO signaling provides a therapeutic window where treatment with EPO may be most efficacious [76]. Altogether, the considerable changes in EPOR expression within the fetal brain in the course of inflammation stresses the need for biomarkers to determine the onset of intra-amniotic infections.

One important limitation of a large animal study is the relative low number of animals per group. Given the relatively small animal numbers per group, we reported actual $p$-values and tended to interpret $p$-values between 0.05 and 0.1 as biologically relevant. This assumption decreases the chance of a false-negative finding but increases the chance that one of these differences is a false-positive result.

\section{Conclusion}

Altogether, the cerebral changes as found in our translational model of inflammation-related brain injury of the preterm infant could be reasonably expected to lead to deficits in learning, memory and social skills and/ or motor disabilities that can persist into adulthood [34]. However, these outcomes need to be confirmed in longitudinal studies, incorporating behavioral analysis and MRI but nonetheless inform the development of interventions to protect and/or regenerate the preterm brain in order to allow normal development. By gaining more insight into the temporal processes underlying inflammation-induced preterm brain injury we provide an increased understanding of the pathophysiological changes in the fetal brain with the goal of helping to design new interventions or implement interventions more effectively to prevent perinatal brain damage. 


\section{References}

1. Rovira N, Alarcon A, Iriondo M, Ibanez M, Poo P, Cusi V et al. Impact of histological chorioamnionitis, funisitis and clinical chorioamnionitis on neurodevelopmental outcome of preterm infants. Early human development. 2011;87(4):253-7. doi:10.1016/j. earlhumdev.2011.01.024.

2. Paton MCB, McDonald CA, Allison BJ, Fahey MC, Jenkin G, Miller SL. Perinatal Brain Injury As a Consequence of Preterm Birth and Intrauterine Inflammation: Designing Targeted Stem Cell Therapies. Frontiers in neuroscience. 2017;11:200. doi:10.3389/fnins.2017.00200.

3. Goncalves LF, Chaiworapongsa T, Romero R. Intrauterine infection and prematurity. Mental retardation and developmental disabilities research reviews. 2002;8(1):3-13. doi:10.1002/mrdd.10008.

4. Kuypers E, Wolfs TG, Collins JJ, Jellema RK, Newnham JP, Kemp MW et al. Intraamniotic lipopolysaccharide exposure changes cell populations and structure of the ovine fetal thymus. Reproductive sciences. 2013;20(8):946-56. doi:10.1177/1933719112472742.

5. Lai JCY, Rocha-Ferreira E, Ek CJ, Wang X, Hagberg H, Mallard C. Immune responses in perinatal brain injury. Brain, behavior, and immunity. 2017;63:210-23. doi:10.1016/j. bbi.2016.10.022.

6. Malaeb S, Dammann O. Fetal inflammatory response and brain injury in the preterm newborn. Journal of child neurology. 2009;24(9):1119-26. doi:10.1177/0883073809338066.

7. Jin C, Londono I, Mallard C, Lodygensky GA. New means to assess neonatal inflammatory brain injury. Journal of neuroinflammation. 2015;12:180. doi:10.1186/s12974-015-0397-2.

8. Gussenhoven R, Ophelders D, Kemp MW, Payne MS, Spiller OB, Beeton ML et al. The Paradoxical Effects of Chronic Intra-Amniotic Ureaplasma parvum Exposure on Ovine Fetal Brain Development. Developmental neuroscience. 2017. doi:10.1159/000479021.

9. Fleiss B, Tann CJ, Degos V, Sigaut S, Van Steenwinckel J, Schang AL et al. Inflammationinduced sensitization of the brain in term infants. Developmental medicine and child neurology. 2015;57 Suppl 3:17-28. doi:10.1111/dmcn.12723.

10. Hagberg H, Dammann O, Mallard C, Leviton A. Preconditioning and the developing brain. Seminars in perinatology. 2004;28(6):389-95.

11. Tsai PT, Ohab JJ, Kertesz N, Groszer M, Matter C, Gao J et al. A critical role of erythropoietin receptor in neurogenesis and post-stroke recovery. The Journal of neuroscience : the official journal of the Society for Neuroscience. 2006;26(4):1269-74. doi:10.1523/JNEUROSCI.448005.2006 . 
12. Yu X, Shacka JJ, Eells JB, Suarez-Quian C, Przygodzki RM, Beleslin-Cokic B et al. Erythropoietin receptor signalling is required for normal brain development. Development. 2002;129(2):505-16.

13. Song J, Sun H, Xu F, Kang W, Gao L, Guo J et al. Recombinant human erythropoietin improves neurological outcomes in very preterm infants. Annals of neurology. 2016;80(1):2434. doi:10.1002/ana.24677.

14. Leuchter RH, Gui L, Poncet A, Hagmann C, Lodygensky GA, Martin E et al. Association between early administration of high-dose erythropoietin in preterm infants and brain MRI abnormality at term-equivalent age. Jama. 2014;312(8):817-24. doi:10.1001/jama.2014.9645.

15. Fauchere JC, Koller BM, Tschopp A, Dame C, Ruegger C, Bucher HU et al. Safety of Early High-Dose Recombinant Erythropoietin for Neuroprotection in Very Preterm Infants. The Journal of pediatrics. 2015;167(1):52-7 e1-3. doi:10.1016/j.jpeds.2015.02.052.

16. Juul SE, McPherson RJ, Bauer LA, Ledbetter KJ, Gleason CA, Mayock DE. A phase I/II trial of high-dose erythropoietin in extremely low birth weight infants: pharmacokinetics and safety. Pediatrics. 2008;122(2):383-91. doi:10.1542/peds.2007-2711.

17. Wu YW, Mathur AM, Chang T, McKinstry RC, Mulkey SB, Mayock DE et al. High-Dose Erythropoietin and Hypothermia for Hypoxic-Ischemic Encephalopathy: A Phase II Trial. Pediatrics. 2016;137(6). doi:10.1542/peds.2016-0191.

18. Wassink G, Davidson JO, Dhillon SK, Fraser M, Galinsky R, Bennet L et al. Partial white and grey matter protection with prolonged infusion of recombinant human erythropoietin after asphyxia in preterm fetal sheep. Journal of cerebral blood flow and metabolism : official journal of the International Society of Cerebral Blood Flow and Metabolism. 2017;37(3):1080-94. doi:10.1177/0271678X16650455.

19. Gao R, Tang YH, Tong JH, Yang JJ, Ji MH, Zhu SH. Systemic Lipopolysaccharide Administration-Induced Cognitive Impairments are Reversed by Erythropoietin Treatment in Mice. Inflammation. 2015;38(5):1949-58. doi:10.1007/s10753-015-0175-4.

20. Rees S, Hale N, De Matteo R, Cardamone L, Tolcos M, Loeliger M et al. Erythropoietin is neuroprotective in a preterm ovine model of endotoxin-induced brain injury. Journal of neuropathology and experimental neurology. 2010;69(3):306-19. doi:10.1097/ NEN.0b013e3181d27138.

21. Wang H, Zhang L, Jin Y. A meta-analysis of the protective effect of recombinant human erythropoietin (rhEPO) for neurodevelopment in preterm infants. Cell biochemistry and biophysics. 2015;71(2):795-802. doi:10.1007/s12013-014-0265-1.

22. Korzeniewski SJ, Allred E, Logan JW, Fichorova RN, Engelke S, Kuban KC et al. Elevated endogenous erythropoietin concentrations are associated with increased risk of brain damage in extremely preterm neonates. PloS one. 2015;10(3):e0115083. doi:10.1371/journal.pone.0115083.

23. NatalucciG, LatalB,KollerB,RueggerC,SickB,HeldLetal.Effect ofEarlyProphylacticHigh-Dose Recombinant Human Erythropoietin in Very Preterm Infants on Neurodevelopmental Outcome at 2 Years: A Randomized Clinical Trial. Jama. 2016;315(19):2079-85. doi:10.1001/ jama.2016.5504. 
24. Marti HH. Erythropoietin and the hypoxic brain. The Journal of experimental biology. 2004;207(Pt 18):3233-42. doi:10.1242/jeb.01049.

25. van der Kooij MA, Groenendaal F, Kavelaars A, Heijnen CJ, van Bel F. Neuroprotective properties and mechanisms of erythropoietin in in vitro and in vivo experimental models for hypoxia/ischemia. Brain research reviews. 2008;59(1):22-33. doi:10.1016/j. brainresrev.2008.04.007.

26. Jantzie LL, Miller RH, Robinson S. Erythropoietin signaling promotes oligodendrocyte development following prenatal systemic hypoxic-ischemic brain injury. Pediatric research. 2013;74(6):658-67. doi:10.1038/pr.2013.155.

27. Kramer BW, Moss TJ, Willet KE, Newnham JP, Sly PD, Kallapur SG et al. Dose and time response after intraamniotic endotoxin in preterm lambs. American journal of respiratory and critical care medicine. 2001;164(6):982-8. doi:10.1164/ ajrccm.164.6.2103061.

28. Newnham JP, Kallapur SG, Kramer BW, Moss TJ, Nitsos I, Ikegami M et al. Betamethasone effects on chorioamnionitis induced by intra-amniotic endotoxin in sheep. American journal of obstetrics and gynecology. 2003;189(5):1458-66.

29. Back SA, Riddle A, Dean J, Hohimer AR. The instrumented fetal sheep as a model of cerebral white matter injury in the premature infant. Neurotherapeutics : the journal of the American Society for Experimental NeuroTherapeutics. 2012;9(2):359-70. doi:10.1007/s13311-012-0108-y.

30. Gavilanes AW, Strackx E, Kramer BW, Gantert M, Van den Hove D, Steinbusch H et al. Chorioamnionitis induced by intraamniotic lipopolysaccharide resulted in an interval-dependent increase in central nervous system injury in the fetal sheep. American journal of obstetrics and gynecology. 2009;200(4):437 e1-8. doi:10.1016/j. ajog.2008.12.003.

31. Jellema RK, Lima Passos V, Zwanenburg A, Ophelders DR, De Munter S, Vanderlocht $\mathrm{J}$ et al. Cerebral inflammation and mobilization of the peripheral immune system following global hypoxia-ischemia in preterm sheep. Journal of neuroinflammation. 2013;10:13. doi:10.1186/1742-2094-10-13.

32. Bashiri A, Burstein E, Mazor M. Cerebral palsy and fetal inflammatory response syndrome: a review. Journal of perinatal medicine. 2006;34(1):5-12. doi:10.1515/JPM.2006.001.

33. Duggan PJ, Maalouf EF, Watts TL, Sullivan MH, Counsell SJ, Allsop J et al. Intrauterine T-cell activation and increased proinflammatory cytokine concentrations in preterm infants with cerebral lesions. Lancet. 2001;358(9294):1699-700.

34. Hagberg H, Gressens P, Mallard C. Inflammation during fetal and neonatal life: implications for neurologic and neuropsychiatric disease in children and adults. Annals of neurology. 2012;71(4):444-57. doi:10.1002/ana.22620.

35. Rosell A, Cuadrado E, Alvarez-Sabin J, Hernandez-Guillamon M, Delgado P, Penalba A et al. Caspase-3 is related to infarct growth after human ischemic stroke. Neuroscience letters. 2008;430(1):1-6. doi:10.1016/j.neulet.2007.05.006. 
36. Volpe JJ, Kinney HC, Jensen FE, Rosenberg PA. The developing oligodendrocyte: key cellular target in brain injury in the premature infant. International journal of developmental neuroscience : the official journal of the International Society for Developmental Neuroscience. 2011;29(4):423-40. doi:10.1016/j. ijdevneu.2011.02.012.

37. Dean JM, van de Looij Y, Sizonenko SV, Lodygensky GA, Lazeyras F, Bolouri H et al. Delayed cortical impairment following lipopolysaccharide exposure in preterm fetal sheep. Annals of neurology. 2011;70(5):846-56. doi:10.1002/ana.22480.

38. Mottahedin A, Ardalan M, Chumak T, Riebe I, Ek J, Mallard C. Effect of Neuroinflammation on Synaptic Organization and Function in the Developing Brain: Implications for Neurodevelopmental and Neurodegenerative Disorders. Frontiers in cellular neuroscience. 2017;11:190. doi:10.3389/fncel.2017.00190.

39. Volpe JJ. The encephalopathy of prematurity--brain injury and impaired brain development inextricably intertwined. Seminars in pediatric neurology. 2009;16(4):16778. doi:10.1016/j.spen.2009.09.005.

40. Czikk MJ, Totten S, Hammond R, Richardson BS. Microtubule-associated protein 2 and synaptophysin in the preterm and near-term ovine fetal brain and the effect of intermittent umbilical cord occlusion. Reproductive sciences. 2015;22(3):367-76. doi:10.1177/1933719114529371.

41. Lu HY, Zhang Q, Wang QX, Lu JY. Contribution of Histologic Chorioamnionitis and Fetal Inflammatory Response Syndrome to Increased Risk of Brain Injury in Infants With Preterm Premature Rupture of Membranes. Pediatric neurology. 2016;61:94-8 e1. doi:10.1016/j.pediatrneurol.2016.05.001.

42. Hagberg H, Mallard C, Ferriero DM, Vannucci SJ, Levison SW, Vexler ZS et al. The role of inflammation in perinatal brain injury. Nature reviews Neurology. 2015;11(4):192208. doi:10.1038/nrneurol.2015.13.

43. Bartha AI, Foster-Barber A, Miller SP, Vigneron DB, Glidden DV, Barkovich AJ et al. Neonatal encephalopathy: association of cytokines with MR spectroscopy and outcome. Pediatric research. 2004;56(6):960-6. doi:10.1203/01.PDR.0000144819.45689.BB.

44. Ramaswamy V, Horton J, Vandermeer B, Buscemi N, Miller S, Yager J. Systematic review of biomarkers of brain injury in term neonatal encephalopathy. Pediatric neurology. 2009;40(3):215-26. doi:10.1016/j.pediatrneurol.2008.09.026.

45. Huang HC, Wang CL, Huang LT, Chuang H, Liu CA, Hsu TY et al. Association of cord blood cytokines with prematurity and cerebral palsy. Early human development. 2004;77(1-2):29-36. doi:10.1016/j.earlhumdev.2004.01.001.

46. Carlo WA, McDonald SA, Tyson JE, Stoll BJ, Ehrenkranz RA, Shankaran S et al. Cytokines and neurodevelopmental outcomes in extremely low birth weight infants. The Journal of pediatrics. 2011;159(6):919-25 e3. doi:10.1016/j.jpeds.2011.05.042.

47. Minghetti L. Cyclooxygenase-2 (COX-2) in inflammatory and degenerative brain diseases. Journal of neuropathology and experimental neurology. 2004;63(9):901-10. 
48. Chhor V, Le Charpentier T, Lebon S, Ore MV, Celador IL, Josserand J et al. Characterization of phenotype markers and neuronotoxic potential of polarised primary microglia in vitro. Brain, behavior, and immunity. 2013;32:70-85. doi:10.1016/j. bbi.2013.02.005.

49. Shiow LR, Favrais G, Schirmer L, Schang AL, Cipriani S, Andres C et al. Reactive astrocyte COX2-PGE2 production inhibits oligodendrocyte maturation in neonatal white matter injury. Glia. 2017;65(12):2024-37. doi:10.1002/glia.23212.

50. Weaver-Mikaere L, Gunn AJ, Mitchell MD, Bennet L, Fraser M. LPS and TNF alpha modulate AMPA/NMDA receptor subunit expression and induce PGE2 and glutamate release in preterm fetal ovine mixed glial cultures. Journal of neuroinflammation. 2013;10:153. doi:10.1186/1742-2094-10-153.

51. Dave KA, Platel JC, Huang F, Tian D, Stamboulian-Platel S, Bordey A. Prostaglandin E2 induces glutamate release from subventricular zone astrocytes. Neuron glia biology. 2010;6(3):201-7. doi:10.1017/S1740925X10000244.

52. Evrard P, Gressens P, Volpe JJ. New concepts to understand the neurological consequences of subcortical lesions in the premature brain. Biology of the neonate. 1992;61(1):1-3.

53. Baburamani AA, Supramaniam VG, Hagberg H, Mallard C. Microglia toxicity in preterm brain injury. Reproductive toxicology. 2014;48:106-12. doi:10.1016/j. reprotox.2014.04.002.

54. Verney C, Pogledic I, Biran V, Adle-Biassette H, Fallet-Bianco C, Gressens P. Microglial reaction in axonal crossroads is a hallmark of noncystic periventricular white matter injury in very preterm infants. Journal of neuropathology and experimental neurology. 2012;71(3):251-64. doi:10.1097/NEN.0b013e3182496429.

55. Billiards SS, Haynes RL, Folkerth RD, Borenstein NS, Trachtenberg FL, Rowitch DH et al. Myelin abnormalities without oligodendrocyte loss in periventricular leukomalacia. Brain pathology. 2008;18(2):153-63. doi:10.1111/j.1750-3639.2007.00107.x.

56. Haynes RL, Folkerth RD, Keefe RJ, Sung I, Swzeda LI, Rosenberg PA et al. Nitrosative and oxidative injury to premyelinating oligodendrocytes in periventricular leukomalacia. Journal of neuropathology and experimental neurology. 2003;62(5):441-50.

57. Back SA, Luo NL, Mallinson RA, O'Malley JP, Wallen LD, Frei B et al. Selective vulnerability of preterm white matter to oxidative damage defined by F2-isoprostanes. Annals of neurology. 2005;58(1):108-20. doi:10.1002/ana.20530.

58. Favrais G, van de Looij Y, Fleiss B, Ramanantsoa N, Bonnin P, Stoltenburg-Didinger $\mathrm{G}$ et al. Systemic inflammation disrupts the developmental program of white matter. Annals of neurology. 2011;70(4):550-65. doi:10.1002/ana.22489.

59. Rousset CI, Chalon S, Cantagrel S, Bodard S, Andres C, Gressens P et al. Maternal exposure to LPS induces hypomyelination in the internal capsule and programmed cell death in the deep gray matter in newborn rats. Pediatric research. 2006;59(3):42833. doi:10.1203/01.pdr.0000199905.08848.55. 
60. Krishnan ML, Van Steenwinckel J, Schang AL, Yan J, Arnadottir J, Le Charpentier T et al. Integrative genomics of microglia implicates DLG4 (PSD95) in the white matter development of preterm infants. Nature communications. 2017;8(1):428. doi:10.1038/s41467-017-00422-w.

61. Tay TL, Savage JC, Hui CW, Bisht K, Tremblay ME. Microglia across the lifespan: from origin to function in brain development, plasticity and cognition. The Journal of physiology. 2017;595(6):1929-45. doi:10.1113/JP272134.

62. Ball G, Srinivasan L, Aljabar P, Counsell SJ, Durighel G, Hajnal JV et al. Development of cortical microstructure in the preterm human brain. Proceedings of the National Academy of Sciences of the United States of America. 2013;110(23):9541-6. doi:10.1073/ pnas.1301652110.

63. Cotter D, Wilson S, Roberts E, Kerwin R, Everall IP. Increased dendritic MAP2 expression in the hippocampus in schizophrenia. Schizophrenia research. 2000;41(2):313-23.

64. Kadoyama K, Takahashi Y, Higashida H, Tanabe T, Yoshimoto T. Cyclooxygenase-2 stimulates production of amyloid beta-peptide in neuroblastoma $\mathrm{x}$ glioma hybrid NG108-15 cells. Biochemical and biophysical research communications. 2001;281(2):48390. doi:10.1006/bbrc.2001.4357.

65. O'Gorman RL, Bucher HU, Held U, Koller BM, Huppi PS, Hagmann CF et al. Tractbased spatial statistics to assess the neuroprotective effect of early erythropoietin on white matter development in preterm infants. Brain : a journal of neurology. 2015;138(Pt 2):388-97. doi:10.1093/brain/awu363.

66. Ohls RK, Cannon DC, Phillips J, Caprihan A, Patel S, Winter S et al. Preschool Assessment of Preterm Infants Treated With Darbepoetin and Erythropoietin. Pediatrics. 2016;137(3):e20153859. doi:10.1542/peds.2015-3859.

67. Neubauer AP, Voss W, Wachtendorf M, Jungmann T. Erythropoietin improves neurodevelopmental outcome of extremely preterm infants. Annals of neurology. 2010;67(5):657-66. doi:10.1002/ana.21977.

68. Zhu C, Kang W, Xu F, Cheng X, Zhang Z, Jia L et al. Erythropoietin improved neurologic outcomes in newborns with hypoxic-ischemic encephalopathy. Pediatrics. 2009;124(2):e218-26. doi:10.1542/peds.2008-3553.

69. Brown MS, Eichorst D, Lala-Black B, Gonzalez R. Higher cumulative doses of erythropoietin and developmental outcomes in preterm infants. Pediatrics. 2009;124(4):e681-7. doi:10.1542/peds.2008-2701.

70. McAdams RM, McPherson RJ, Mayock DE, Juul SE. Outcomes of extremely low birth weight infants given early high-dose erythropoietin. Journal of perinatology : official journal of the California Perinatal Association. 2013;33(3):226-30. doi:10.1038/ jp.2012.78.

71. Bierer R, Peceny MC, Hartenberger CH, Ohls RK. Erythropoietin concentrations and neurodevelopmental outcome in preterm infants. Pediatrics. 2006;118(3):e635-40. doi:10.1542/peds.2005-3186. 
72. El Shimi MS, Awad HA, Hassanein SM, Gad GI, Imam SS, Shaaban HA et al. Single dose recombinant erythropoietin versus moderate hypothermia for neonatal hypoxic ischemic encephalopathy in low resource settings. The journal of maternal-fetal \& neonatal medicine : the official journal of the European Association of Perinatal Medicine, the Federation of Asia and Oceania Perinatal Societies, the International Society of Perinatal Obstet. 2014;27(13):1295-300. doi:10.3109/14767058.2013.855894.

73. Nguyen AQ, Cherry BH, Scott GF, Ryou MG, Mallet RT. Erythropoietin: powerful protection of ischemic and post-ischemic brain. Experimental biology and medicine. 2014;239(11):1461-75. doi:10.1177/1535370214523703.

74. Castillo-Melendez M, Yan E, Walker DW. Expression of erythropoietin and its receptor in the brain of late-gestation fetal sheep, and responses to asphyxia caused by umbilical cord occlusion. Developmental neuroscience. 2005;27(2-4):220-7. doi:10.1159/000085995.

75. Taoufik E, Petit E, Divoux D, Tseveleki V, Mengozzi M, Roberts ML et al. TNF receptor I sensitizes neurons to erythropoietin- and VEGF-mediated neuroprotection after ischemic and excitotoxic injury. Proceedings of the National Academy of Sciences of the United States of America. 2008;105(16):6185-90. doi:10.1073/pnas.0801447105.

76. Brines M, Cerami A. The receptor that tames the innate immune response. Molecular medicine. 2012;18:486-96. doi:10.2119/molmed.2011.00414. 
3 
Ruth Gussenhoven*, Daan RMG

Ophelders*, Martin Lammens, Benno

Küsters, Matthew W Kemp, John

P Newnham, Matthew S Payne,

Suhas G Kallapur, Allan H Jobe, Luc J

Zimmermann, Boris W Kramer, Tim GAM Wolfs

Journal of Neuroinflammation. 2016;13[1]:1

*Authors contributed equally 


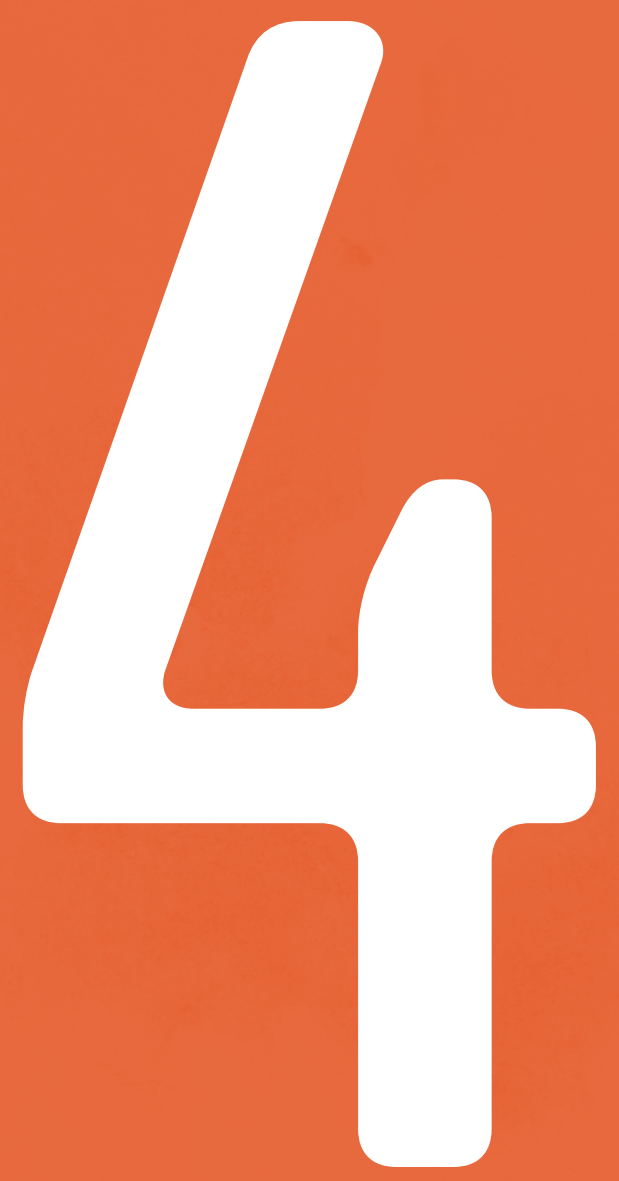

Neuroinflammation and structural injury of the fetal ovine brain following intra-amniotic Candida albicans exposure. 


\section{Abstract}

\section{Background}

Intra-amniotic Candida albicans infection is associated with preterm birth and high morbidity and mortality rates. Survivors are prone to adverse neurodevelopmental outcomes. The mechanisms leading to these adverse neonatal brain outcomes remain largely unknown. To better understand the mechanisms underlying Candida albicans-induced fetal brain injury, we studied immunological responses and structural changes of the fetal brain in a well-established translational ovine model of intra-amniotic Candida albicans infection. In addition, we tested whether these potential adverse outcomes of the fetal brain were improved in utero by antifungal treatment with Fluconazole.

\section{Methods}

Pregnant ewes received an intra-amniotic injection of 107 colony forming units Candida albicans or saline (controls) at 3 or 5 days before preterm delivery at 0.8 of gestation (term 150d). Fetal intra-amniotic/intraperitoneal injections of Fluconazole or saline (controls) were administered 2 days after Candida albicans exposure. Post mortem analyses for fungal burden, peripheral immune activation, neuroinflammation and white matter/ neuronal injury were

\section{Results}

Intra-amniotic exposure to Candida albicans caused a severe systemic inflammatory response, illustrated by a robust increase of plasma IL-6 concentrations. Cerebrospinal fluid cultures were positive for Candida albicans in the majority of the 3 day Candida albicans-exposed animals whereas no positive cultures were present in the 5 day Candida albicansexposed and Fluconazole treated animals. Although Candida albicans was not detected in the brain parenchyma, a neuroinflammatory response in the hippocampus and white matter was seen which was characterized by increased microglial and astrocyte activation. These neuroinflammatory changes were accompanied by structural white matter injury. Intra- 
amniotic Fluconazole reduced fetal mortality, but did not attenuate neuroinflammation and white matter injury.

\section{Conclusion}

Intra-amniotic Candida albicans exposure provoked acute systemic and neuroinflammatory responses with concomitant white matter injury. Fluconazole treatment prevented systemic inflammation without attenuating cerebral inflammation and injury. 


\section{Background}

Preterm birth is associated with chorioamnionitis which is defined as inflammation of the fetal membranes and amniotic fluid caused by microbial invasion [1,2]. The microorganisms most frequently associated with this condition include Ureaplasma species, Mycoplasma hominis, and Gardnerella vaginalis all of which most commonly originate from the lower reproductive tract [3]. These microorganisms and/or inflammatory mediators in the amniotic cavity can cause a fetal inflammatory response syndrome (FIRS) [4-6]. Chorioamnionitis and subsequent FIRS are independent risk factors for adverse outcomes, including injury of the fetal brain [5, 7]. Adverse neurodevelopmental outcomes result from diffuse cerebral inflammation and white matter injury, periventricular leukomalacia and intraventricular hemorrhage [5, 8, 9]. These conditions are associated with a high mortality rate and survivors are predisposed to long-term morbidity including mental retardation, impaired learning and visual disorders and in severe cases cerebral palsy $[7,8,10]$.

The pathophysiology of chorioamnionitis can also include viral and fungal species [11, 4, 12]. Candida albicans (C. albicans) is a commensal fungus of the gastro-intestinal tract which can be asymptomatic in the vaginal microbiota with increasing incidence during pregnancy [13, 14]. Intraamniotic $C$. albicans infections are associated with high mortality rates and severely impaired neurodevelopmental outcomes [14, 13, 15, 16] in which the mechanisms linking fetal exposure to neurological pathologies remain essentially unstudied. We hypothesized that antenatal exposure to $C$. albicans caused a neuroinflammatory response and subsequent white matter injury, which we tested by exposing fetal sheep to intra-amniotic $C$. albicans $[17,16]$.

In two clinical cases intra-amniotic C. albicans infections resolved after oral and intra-amniotic Fluconazole [13]. Fluconazole is the most frequently used antifungal in C. albicans infections, and inhibits ergosterol synthesis, which is an essential component of fungal cell membranes [18]. We therefore 
further hypothesized that fetal intra-amniotic and intra-peritoneal administration of Fluconazole would reduce the neuroinflammatory response and subsequent white matter injury to the fetal brain. Accordingly, systemic immune activation, neuroinflammation and structural white matter injury were assessed in the fetal sheep exposed to intra-amniotic C. albicans and treated with Fluconazole.

\section{Methods}

\section{Experimental design}

The study was approved by and performed according to the guidelines of the animal ethics committee of the University of Western Australia (Perth, Australia). Twenty-six date-mated Merino ewes with singleton pregnancies were randomly assigned to receive an intra-amniotic injection of saline (2 $\mathrm{mL}$ ) as a control or C. albicans (107 colony forming units (CFU), Western Australian clinical isolate) [16]. After 2 days an intra-amniotic/-peritoneal injection of Fluconazole (F) (30 mg per injection, Claris Life Sciences Limited, Chacharwadi-vasana, Ahmedabad-382 213, India) or saline (controls) was administered with delivery after 1 and 3 days (Figure 1). Intra-amniotic injections were performed as previously described [17].

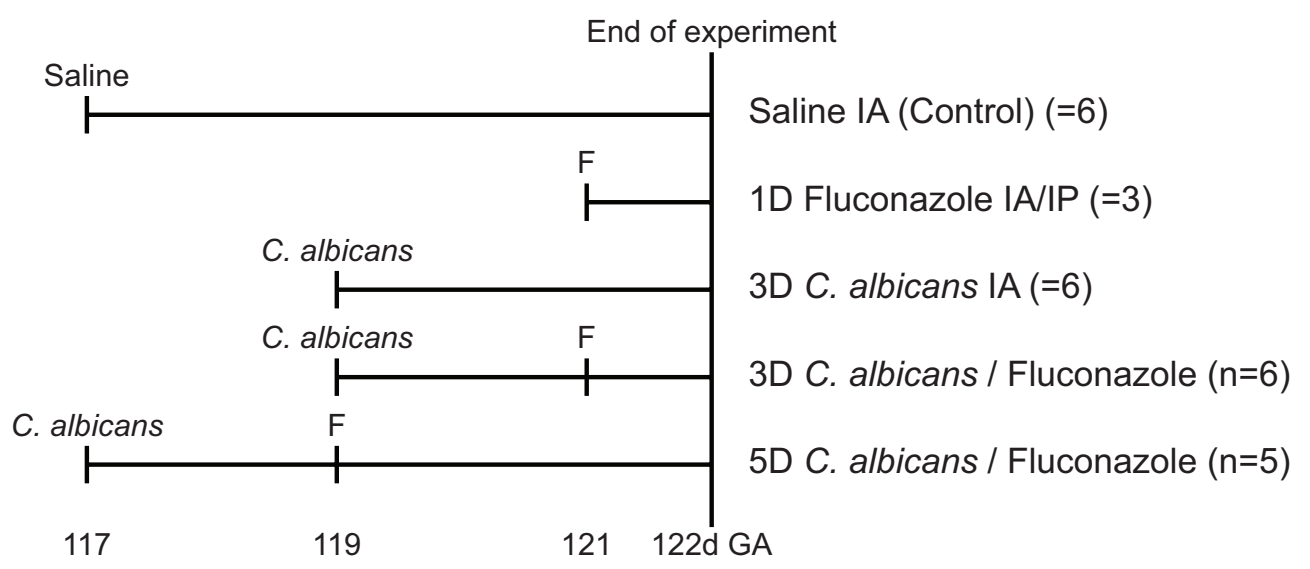

Figure 1. Experimental setup. Animals were exposed to C. albicans for 3 or 5 days in the absence or presence of intra-amniotic (IA) / intraperitoneal (IP) Fluconazole (F). Control animals received intraamniotic (IA) injection with saline. Fetuses were delivered at 0.8 of gestation. 
We did not include a 5D C. albicans only group since previous results with this model indicated that 5 days exposure to $C$. albicans alone was lethal $[16,17]$. Given the survival of 5D C. albicans/Fluconazole-treated group, we have concluded that Fluconazole in this model increases survival [16, 17].

\section{Tissue collection.}

Fetuses were delivered by Caesarean section at 0.8 of gestation (term $~ 150$ days) [19], equivalent of 32 - 34 weeks human gestation [20], and euthanized with intravenous pentobarbitone $(100 \mathrm{mg} / \mathrm{kg}$. Amniotic fluid, blood and cerebrospinal fluid (CSF) were collected at delivery and cultures for C. albicans were performed [16]. Briefly, amniotic fluid $(100 \mu \mathrm{L})$ was inoculated onto Difco Sabaraud-Dextrose agar and incubated at $37^{\circ} \mathrm{C}$ for $48 \mathrm{~h}$. Blood $(2 \mathrm{~mL})$ and liquor $(1 \mathrm{~mL})$ was inoculated into BACTEC Peds Plus culture vials (Becton Dickinson, Frankln Lakes, NJ) and incubated aerobically at $37^{\circ} \mathrm{C}$ for 72 hours [16]. Brains were immersion fixated in $4 \%$ paraformaldehyde.

\section{Enzyme-linked immunosorbent assay IL-6.}

Plasma interleukin (IL)-6 concentrations were assessed as an indication for systemic inflammation using a sheep-specific sandwich enzyme-linked immunosorbent assay (ELISA). First, 96 well plates were pre-coated with $100 \mu \mathrm{L}$ of mouse-anti-ovine monoclonal antibody (MAB1004, Millipore, Darmstadt, Germany, working concentration 1:200) overnight at $4^{\circ} \mathrm{C}$. Plates were washed three times with $0.05 \%$ tween-20 in phosphate buffered saline (PBST) Non-specific binding was blocked with a blocking buffer $(1 \%$ bovine serum albumin (BSA) in PBST) for 1 hour followed by a washing step.

Protein standards were prepared by serial dilution of recombinant IL-6 (ImmunoChemistry Technologies, Bloomington, MN, USA). Plasma samples $(100 \mu \mathrm{L})$ were loaded per well in duplicate and incubated for 2 hours at room temperature. Unbound protein was omitted by washing with PBST, followed by incubation with detection antibody (rabbit-anti-ovine IL-6, AB1839, Millipore, Darmstadt, Germany, working concentration 1:500) for 60 minutes and subsequent washing. Next, each well was incubated for 30 minutes with $100 \mu \mathrm{L}$ of a goat-anti-rabbit-HRP (Jackson ImmunoResearch Laboratories Inc, West Grove, PA, USA, working concentration 1:500) followed by washing and incubation with $3,3^{\prime}, 5,5^{\prime}$-tetramethylbenzidine (TMB) substrate solution for 15 minutes at room temperature. The reaction was stopped by addition of $50 \mu \mathrm{L}$ $2 \mathrm{~N}$ sulfuric acid to each well. Plates were then read on an ELISA plate reader at $450 \mathrm{~nm}$. 


\section{Histology and immunohistochemistry.}

The right hemisphere was divided into 4 defined anatomical regions.

Serial coronal sections of identical thickness $(4 \mu \mathrm{m})$ of the region containing the posterior hippocampus and mid-thalamus were cut with a Leica RM2235 microtome. Within this region, we analyzed hippocampus and the cerebral white matter since there regions are most affected following intra-uterine infection at this developmental stage [21]. All stainings were performed in series (every 15th section) and four sections per staining per animal were analyzed. Hematoxylin and eosin (H\&E) and periodic acid-Schiff (PAS) stainings were performed for morphological and anatomical analysis and for identification of pseudohyphae of $C$. albicans, respectively. Adjacent sections were stained for ionized calcium binding adaptor molecule 1 (IBA-1) (Wako Pure Chemical Industries, Osaka, Japan) for microglia, glial fibrillary acidic protein (GFAP) (DAKO Z0334) for astrocytes, myelin basic protein (MBP) (Merck Millipore, MAB386) for myelin sheaths, 2',3'-Cyclic-nucleotide 3'-phosphodiesterase (CNPase) (Sigma, C5922) for mature myelin producing oligodendrocytes, oligodendrocyte transcription factor 2 (Olig2) (Millipore, AB9610) for oligodendrocyte lineage cells, cluster of differentiation (CD) 68 (DAKO, M0718) for active microglia/phagocytizing macrophages, myeloperoxidase (MPO) (DAKO, A0398) for neutrophils, CD3 (DAKO A0452) for T-lymphocytes, Ki67 (DAKO, M7240) for cell proliferation, and cleaved caspase-3 (Cell signaling, \#9661) for apoptosis.

Sections were deparaffinized and rehydrated. Endogenous peroxidase activity was inactivated with $0.3 \% \mathrm{H}_{2} \mathrm{O}_{2}$ treatment. For every immunolabeled stain with the exception of GFAP and CD68, antigen retrieval was performed by microwave boiling of tissue sections in citrate buffer, $\mathrm{pH}$ 6.0. For CD68, antigen retrieval was performed with proteinase K (DAKO, S3004) treatment for 5 minutes at $37^{\circ} \mathrm{C}$. Nonspecific binding was blocked by incubation with 5\% BSA (MBP, Olig2 and CD3) or goat serum in PBS (IBA-1, GFAP, CNPase, CD68, MPO and Ki67). Sections were incubated overnight at $4^{\circ} \mathrm{C}$ in a closed humidity chamber with primary antibody (IBA-1, GFAP, MBP, CNPase and caspase-3 1:1000; Olig2, MPO and CD3 1:200; Ki67 1:100; CD68 1:50), and subsequently incubated with the specific secondary antibody. Immunostaining was enhanced with a Vectastain $\mathrm{ABC}$ peroxidase Elite kit (Vector Laboratories Inc, Burlingame, CA) followed by a (nickel) 3,3'-diaminobenzidine (DAB) staining and 0,1\% Nuclear Fast Red (Olig2, CD3, Ki67 and caspase-3) or Mayer's Hematoxylin (CD68 and $\mathrm{MPO})$ for background staining. Sections were dehydrated and cover slipped. 
To identify the mature and total number of oligodendrocytes, an immunohistochemical procedure for sequential double staining of CNPase and Olig2 was applied. In this procedure, sections were treated as described above and incubated with the anti- CNPase antibody, followed by incubation with a biotinylated anti-mouse secondary antibody and visualization with 3,3-diaminobenzidine (DAB; Dako). Before incubation with the Olig2 primary antibody, slides were blocked again with 5\% BSA for 60 minutes. Cells that stained positive for Olig2 were visualized by HistoGreen (Linaris, E109).

\section{Immunofluorescence.}

To identify $C$. albicans in the brain tissue, immunofluorescence staining was performed as described previously [17] using a rabbit anti-C. albicans antibody (Meridian Life Science, Memphis, TN, working concentration 1:50) and appropriate Alexa Fluor 594-labelled secondary antibody (working concentration 1:200). Briefly, sections were deparaffinized, rehydrated and incubated in $0.25 \%$ ammonia dissolved in $70 \%$ ethanol for one hour on a shaker. Antigen retrieval was performed using citrate buffer ( $\mathrm{pH}$ 6.0) in a microwave oven. Nonspecific binding was blocked by incubation with $10 \%$ goat serum for one hour followed by overnight incubation with primary anti-C.albicans antibody dissolved in $0.1 \%$ PBS with $0.2 \%$ Tween-20. For fluorescence staining, Alexa Fluor conjugated secondary antibody was used followed by incubation in $0.3 \%$ Sudan Black Solution to reduce auto-fluorescence. Counterstaining was done with the fluorescent nuclear marker Hoechst.

To assess neuronal injury, a Fluoro-Jade $\mathrm{C}$ staining was performed, which is a specific marker for identification of degenerating neurons [22, 23]. In this procedure, sections were deparaffinized, rehydrated and immersed in 0,06\% potassium permanganate with $0,01 \%$ Hoechst for 10 minutes. Before immersing in the Fluoro-Jade $\mathrm{C}$ solution, sections were rinsed in running tap water for 1-2 minutes. Immersion in the Fluoro-Jade C solution (Millipore, AG325) $(0,01 \%$ stock solution in 0,1\% acetic acid) was performed in the dark for 10 minutes. Sections were washed with distilled water and subsequently air dried at $50^{\circ} \mathrm{C}$. Finally, sections were cleared with xylene for 1 minute before cover slipped using DPX.

\section{Qualitative analysis.}

H\&E-stained sections were analyzed by three independent investigators and two neuropathologists, blinded to the experimental groups, to assess overall brain structure and inflammatory changes. The absence of tissue autolysis in our sections was confirmed by neuropathologists. The sections were examined 
for the presence of hemorrhages, gliosis, (cytotoxic) edema, abscess formation, and structural damage, including cyst formation. C. albicans fluorescent and PAS-stained sections were examined for the presence of C. albicans.

\section{Quantitative analysis.}

For the analysis of IBA-1, GFAP, MBP, CNPase, Olig2, and CD68 immunoreactivity, digital images of the hippocampus, white matter (WM) and periventricular white matter (PVWM) were acquired using an Olympus AX-70 microscope (Olympus, Tokyo, Japan) equipped with a black and white digital camera. From each section, one picture of the hippocampus was taken at a 20x magnification and images in the WM were taken at a 100x magnification of the gliotic foci and 4-6 consecutive images of the PVWM. The area fraction of IBA-1, GFAP and MBP immuno-reactivity was determined with a standard intensity threshold to determine positive staining using Qwin Pro V 3.5.1 software (Leica, Rijswijk, The Netherlands). Blood vessels and artefacts were excluded from analysis. The CNPase and Olig2 positive cells were counted using Qwin software and expressed as cells/mm2. Ki67, caspase-3, CD3 and MPO positive cells were counted in all brain regions, focusing on the cerebral vasculature. The digital images were acquired and analyzed by an independent observer who was blinded to the experimental groups. Fluoro-Jade C/Hoechst stainings were examined using the Olympus AX-70 fluorescent microscope.

\section{Data analysis.}

Statistical analysis was performed with GraphPad Prism software (version v5.0; GraphPad Software Inc., La Jolla, CA, USA). Results were analyzed using the non-parametric Kruskal-Wallis test with Dunnett's post-hoc testing to compare different groups. Results are given as mean and standard error of the mean (SEM) with significance at $\mathrm{p}<0.05$. 


\section{Results}

\section{C. albicans culture.}

C. albicans colonial morphology was confirmed by growth on Brilliance Candida Agar (Oxoid, Adelaide, Australia). Positive cerebrospinal fluid (CSF) cultures for C. albicans were detected in 67\% (4/6) of the 3D C. albicansexposed animals and in 50\% (3/6) of the 3D C. albicans/ Fluconazole-treated animals (Table 1). No positive cultures were present in the 5D C. albicans/ Fluconazole-treated group (0/5). Control animals had negative CSF cultures. Results of amniotic fluid and blood cultures were previously published by Maneenil and colleagues and are also summarized in the table [16].

\begin{tabular}{lllll}
\hline & Control & 3D C. albicans & $\begin{array}{l}\text { 3D C. albicans } \\
\text { and Fluconazole }\end{array}$ & $\begin{array}{l}\text { 5D C. albicans } \\
\text { and Fluconazole }\end{array}$ \\
$\begin{array}{l}\text { Cerebrospinal } \\
\text { fluid }\end{array}$ & $3021 \pm 328$ & $2765 \pm 312$ & $2256 \pm 305^{*}$ & $3040 \pm 285$ \\
Fetal blood * & $52.2 \pm 4.5$ & $50.8 \pm 4.9$ & $46.0 \pm 2.8 \#$ & $51.7 \pm 7.0$ \\
Amniotic fluid * $^{*}$ & $1.7 \pm 0.02$ & $1.9 \pm 0.02$ & $2.1 \pm 0.02^{*}$ & $1.7 \pm 0.02$ \\
\hline
\end{tabular}

Table 1. Candida albicans cultures. The ratio of C. albicans positive / total cultures are depicted. * Based on previously published data [16].

\section{No evidence of $C$. albicans invasion in the brain.}

Despite substantial numbers of animals with positive CSF cultures for $C$. albicans, PAS and immunofluorescent staining did not identify C. albicans in the parenchyma of the brains of any of the experimental groups (data not shown).

\section{Pro-inflammatory cytokine response.}

Significantly elevated plasma IL-6 concentrations were found in 50\% (3/6) of the 3D C. albicans-exposed animals when compared to controls (Figure 2). $40 \%(2 / 5)$ of the 5D C. albicans/Fluconazole-treated animals demonstrated elevated plasma concentrations of IL-6, however statistical significance was not reached. Plasma IL-6 concentrations in the controls, 1D Fluconazoleand 3D C. albicans/Fluconazole-treated animals were not detectable. 


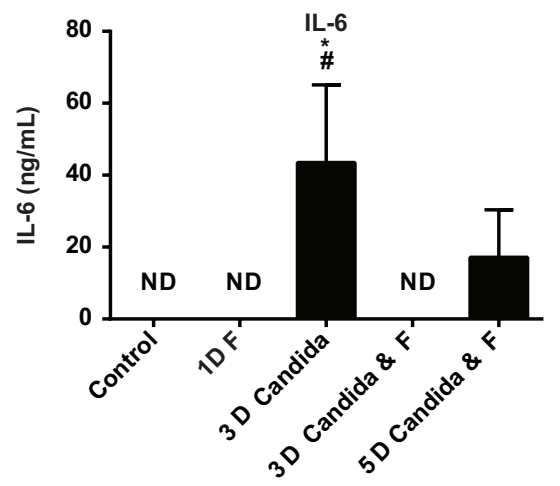

Figure 2. Plasma IL-6 concentrations following intra-amniotic $C$. albicans and/or Fluconazole treatment. For statistical analysis, undetectable values were assigned an arbitrary value of $1 \mathrm{pg} /$ $\mathrm{mL}$. * $\mathrm{P} \leq 0.05$ vs. control; \# $\mathrm{P} \leq 0.05$ vs. $3 \mathrm{D}$ C. albicans/ Fluconazole.

\section{Qualitative analysis of the brain.}

Qualitative analysis of H\&E stained sections revealed no evidence of structural changes including intraventricular hemorrhages and cystic lesions in any of the experimental groups. However, the brains of $C$. albicans-exposed animals had increased cell density in the white matter which primarily consisted of glial cells and blood-derived macrophages, that cannot be distinguished from microglia (Figure 3). These gliotic lesions were primarily present in the gyral crests of the white matter and were more pronounced in $100 \%$ (5/5) of animals of the 5D C. albicans/ Fluconazole-treated group (Figure 3 C-D), and in 67\% (8/12) of animals in the 3D C. albicans-exposed groups. Control animals had mild to no gliotic lesions (Figure $3 \mathrm{~A}-\mathrm{B})$. Interestingly, the subcortical white matter was not affected (Figure 3C).
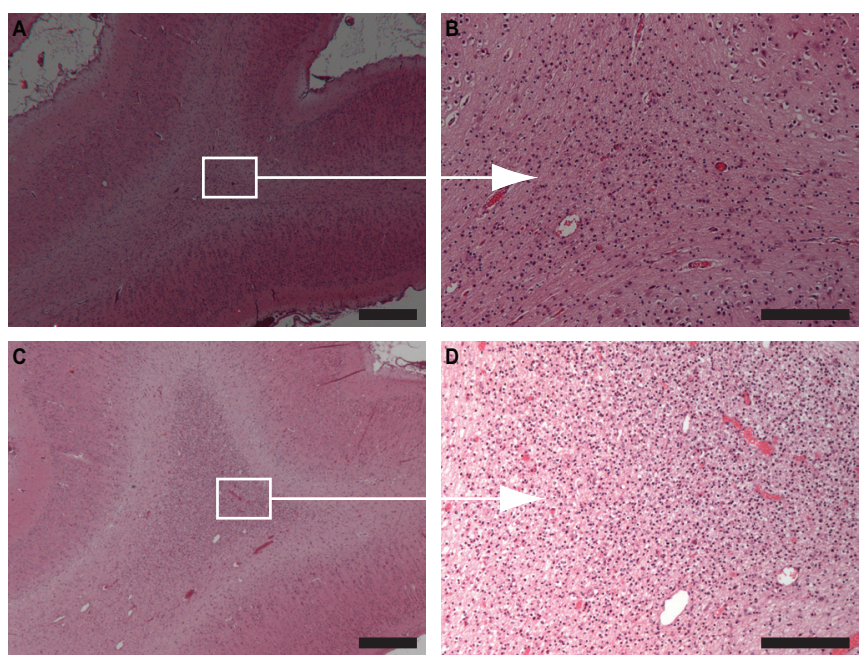

Figure 3. H\&E stained brain sections of control animals and 5D C. albicans/ Fluconazole-treated animals. Control animals $(\mathrm{A}, \mathrm{B})$ showed no to mild gliosis and the 5D C. albicans/Fluconazole-treated animals (C,D) showed severe gliotic foci in white matter of the gyral crest. A and C at 25x magnification (scale bar = $500 \mu \mathrm{m}$ ) and B and D at 200x magnification (scale bar $=100$ $\mu \mathrm{m})$. 

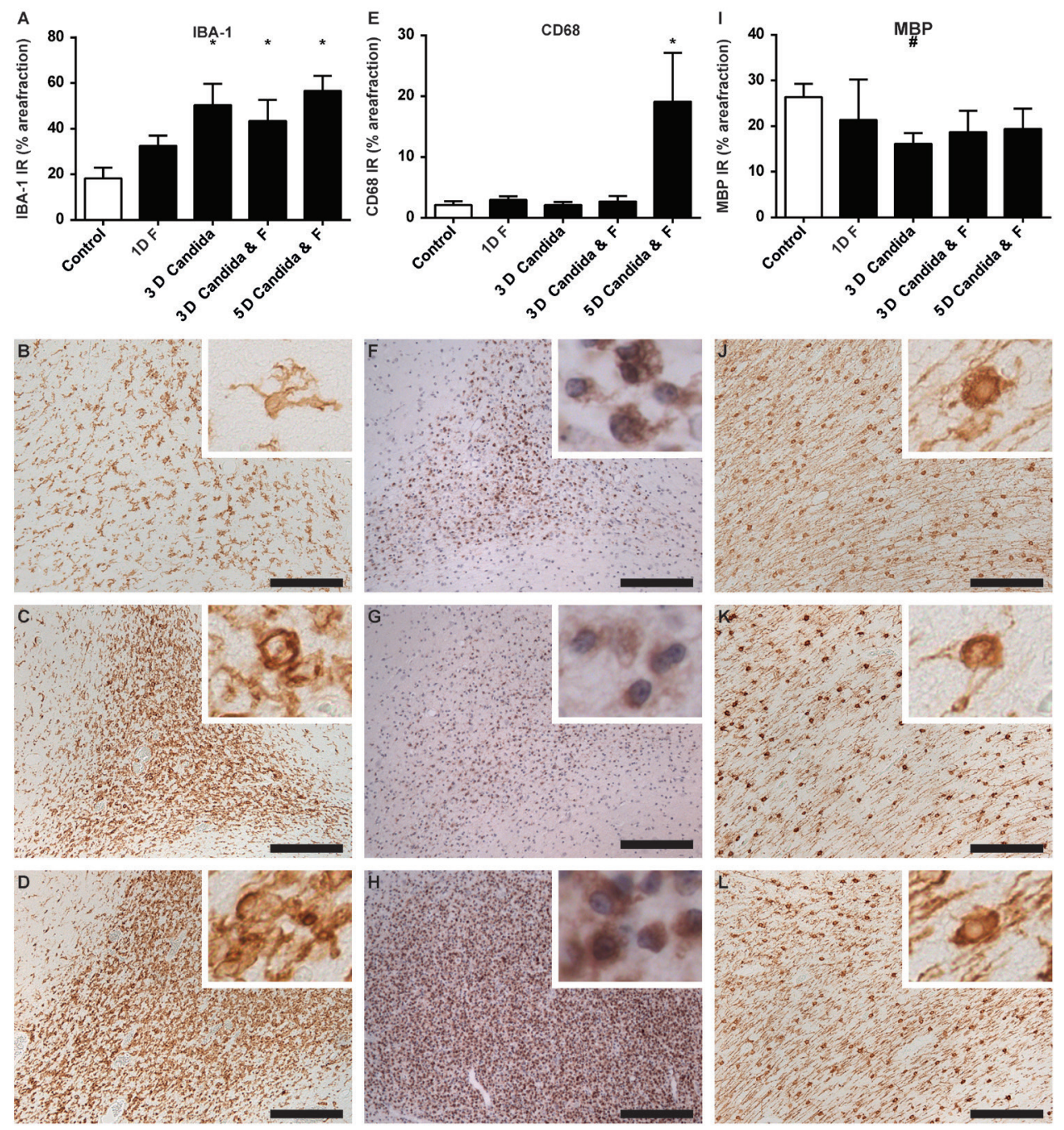

Figure 4. Antenatal exposure to $C$. albicans caused microglia activation and subsequent myelin disturbances in the white matter. Significant increase $\left({ }^{*}, \mathrm{p}<0.05\right)$ of the area fraction of IBA-1 (A-D) immunoreactivity (IR) was observed in all C. albicans-exposed animals compared to controls irrespective of Fluconazole (F) treatment. Significant increase $\left(^{*}, \mathrm{p}<0,05\right)$ of the area fraction of CD68 IR (E-H) was observed in animals of the 5D C. albicans/Fluconazole group. A decrease of MBP IR (I-L) was observed which did not reached significance $(\#, \mathrm{p}<0.1)$ in the 3D C. albicansexposed group compared to the controls irrespective of Fluconazole treatment. Representative figures show that the area fraction of the IBA-1 IR is higher in the 3D C. albicans group (C) and 5D C. albicans/Fluconazole-treated group (D) compared to the control group (B). Morphological analysis showed a higher density of amoeboid microglia present in the white matter after C. albicans exposure (inserts). In these regions with microglia activation, myelin disturbances and loss of myelin fibers were found in the 3D C. albicans-exposed (G) and the 5D C. albicans/Fluconazoletreated $(\mathrm{H})$ group. Figures of the 3D C. albicans/Fluconazole-treated group are not depicted here. Images taken at 100x magnification (insert at 400x magnification), scale bar $=500 \mu \mathrm{m}$. 


\section{Antenatal exposure to C. albicans caused microglia/macrophage and astrocyte activation in the hippocampus and white matter}

The neuroinflammatory changes as indicated by the more pronounced presence of gliotic foci, in C. albicans-exposed animals were further evaluated by immunohistochemical analysis of the microglial marker IBA-1. This staining demonstrated IBA-1 immunoreactivity (IR) distributed throughout the white matter in distinct foci in the $C$. albicans exposed animals. These foci were primarily detected at the gyral crests of the white matter which corresponds with the increased cell density found in the H\&E staining. This finding is substantiated by an increase of IBA-1 IR within these foci after intra-amniotic exposure to C. albicans irrespective of Fluconazole treatment compared to control group and/or 1D Fluconazole group (Figure 4A-D). These foci comprised microglia of both ramified and amoeboid morphology. However, quantification of microglial activation based on microglial morphology was hampered by the high cell density within these foci. Immunoreactivity of CD68, representative for activated microglia/phagocytosing macrophages [24], was increased in the 5D C. albicans/Fluconazole group compared to controls (Figure 4 E-H). No significant changes in IBA-1 immunoreactivity in the periventricular white matter were observed. However, in 17\% (1/6) of the 3D C. albicans animals and $60 \%(3 / 5)$ of the 5D C. albicans/Fluconazole animals, foci containing CD68 positive cells were found in the periventricular white matter (data not shown). Besides an increase in IBA-1 immunoreactivity, an increase of the astrocyte marker glial fibrillary acidic protein (GFAP) was found in the hippocampus after intra-amniotic exposure to C. albicans compared to the control group and/or 1D Fluconazole group (Figure 5). Administration of Fluconazole irrespective of pre-exposure to $C$. albicans did not induce changes in IBA-1 or GFAP immunoreactivity. 

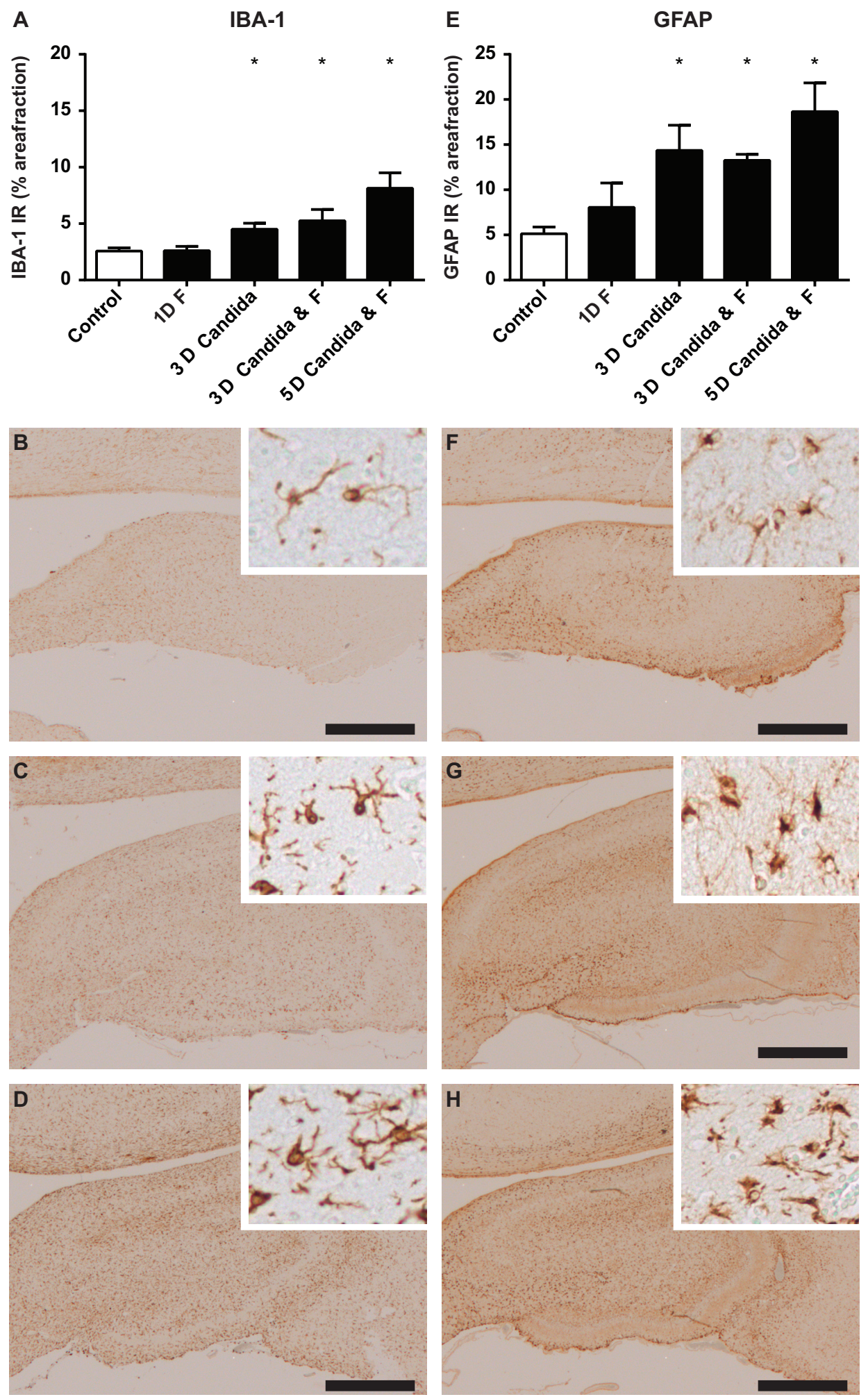
< Figure 5. Antenatal exposure to Candida albicans caused microglial (IBA-1) and astrocyte (GFAP) activation in the hippocampus. A significant increase $\left({ }^{*}, \mathrm{p}<0.05\right)$ of the area fraction of IBA-1 (A-D) and GFAP (E-H) immunoreactivity (IR) was observed in all C. albicans-exposed animals compared to controls irrespective of Fluconazole (F) treatment (A,E). No significant changes were found in the Fluconazole treated animals compared to controls. Representative figures show that the area fraction of the IBA-1 IR and GFAP IR was higher in the 3D C. albicans group $(C, G)$ and 5D C. albicans/Fluconazole-treated group (D,H) compared to the control group (B,F). Images at 20x magnification (inserts at 200x magnification), scale bar $=100 \mu \mathrm{m}$.

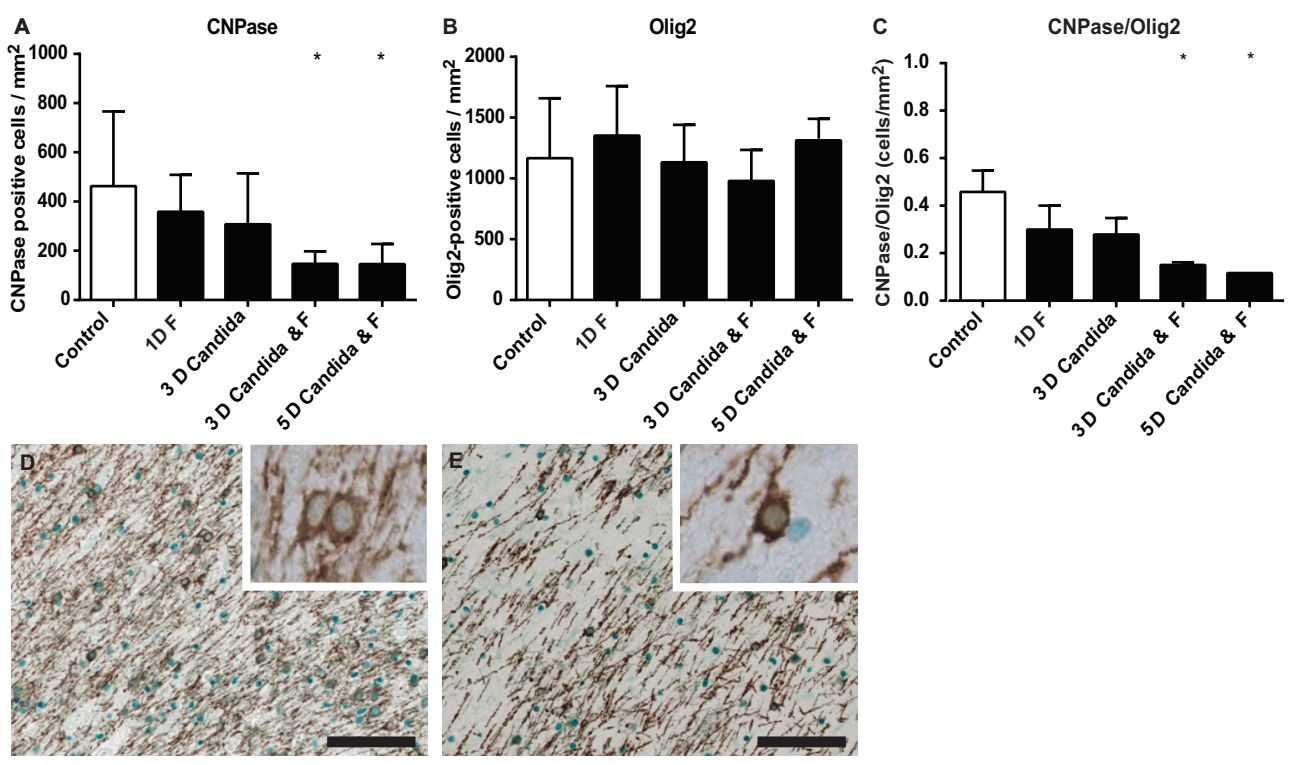

Figure 6. Myelin disturbances are accompanied by a loss of mature oligodendrocytes. A significant decrease $\left({ }^{*}, \mathrm{p}<0.05\right)$ of the ratio between CNPase positive mature oligodendrocytes and total number of cells of oligodendroglial lineage (Olig2 positive) was observed following 3D C. albicans and Fluconazole (F) treatment and 5D C. albicans-exposed groups and Fluconazole treatment compared to controls. $(\mathrm{A}, \mathrm{B})$ Graphical representation of the CNPase (A) and Olig2 (B) data; (C) Graphical representation of the CNPase/Olig2 ratio; (D-E) representative figures of CNPase/Olig2 double stain in (D) controls and (E) 5D C. albicans-exposed animals indicating an apparent loss of CNPase positive cells. Images taken at 200x magnification (insert at 400x magnification), scale bar $=100 \mu \mathrm{m}$.

There was reduced MBP immunoreactivity within regions of overt microglial activation in the 3D C. albicans-exposed animals. In addition, myelin texture was disturbed in all C. albicans-exposed animals whereas no myelin disturbances were observed in control animals (Figure $4 \mathrm{I}-\mathrm{L}$ ). The apparent loss of MBP IR and altered texture of myelin prompted us to further investigate the ratio between mature and total oligodendroglial lineage cells. Double staining for CNPase and Olig2 revealed a significant decrease of CNPase positive cells in the 3D C. albicans/Fluconazole and 5D C. albicans/Fluconazole groups whereas the number of Olig2 positive cells 
remained identical, thereby resulting in a decreased ratio of mature versus total oligodendrocytes in these animals (Figure $6 \mathrm{~A}-\mathrm{E}$ ).

\section{Antenatal exposure to $C$. albicans did not result in neuronal degeneration}

In all experimental groups no Fluoro-Jade $C$ positive neurons were detected throughout the brain sections (data not shown).

\section{MPO and CD3}

MPO- and CD3-positive cells were sporadically detected in the cerebral vasculature of the choroid plexus and meninges. No MPO- and CD3-positive cells were found in the cerebral white matter. No differences were seen between all experimental groups (supplementary figure 1).

\section{Ki67 and caspase 3}

An increase in Ki67 and caspase-3 positive cells was found in the white matter and hippocampus of the 5D C. albicans/Fluconazole group compared to controls (Figure $7 \mathrm{~A}-\mathrm{H}$, Table 2). Moreover, in the periventricular white matter, an increase in caspase-3 positive cells was observed.

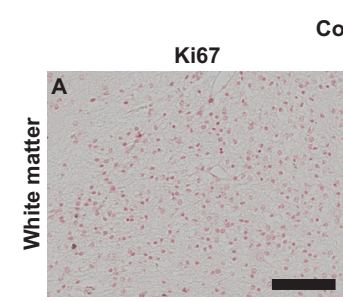

Control
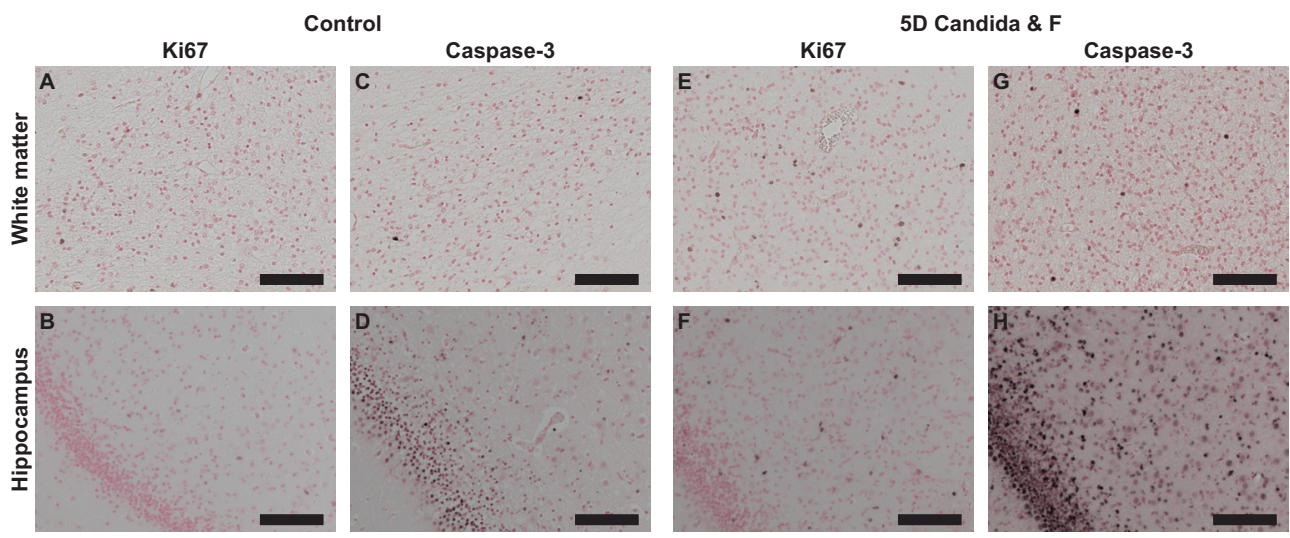

Figure 7. Antenatal exposure to Candida albicans resulted in increased proliferation and apoptosis in the subcortical white matter and hippocampus. A significant increase $\left({ }^{*}, \mathrm{p}<0.05\right)$ of KI67 and caspase-3 positive cells were observed following 5D C. albicans with Fluconazole treatment in the white matter $(\mathrm{E}, \mathrm{G})$ and hippocampus $(\mathrm{F}, \mathrm{H})$ compared to controls (A,B,C,D). Images taken at 200x magnification, scale bar $=100 \mu \mathrm{m}$. 

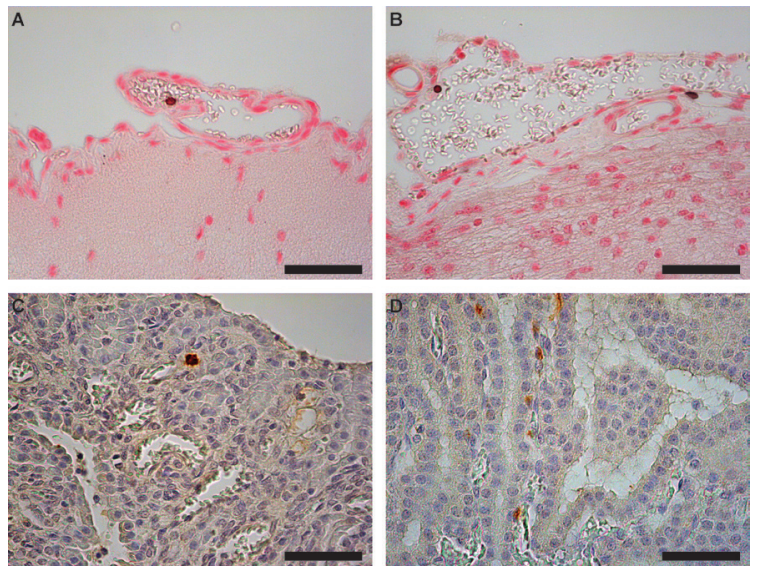

Supplementary figure 1. Antenatal exposure to $C$. albicans does not result in infiltration of peripheral immune cells. (A-B) representative pictures of $\mathrm{CD} 3$ positive $T$ lymphocytes in the meninges of (A) controls and (B) 5D C. albicans / Fluconazole animals. (C-D) representative

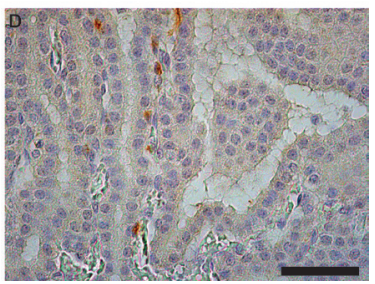
pictures of MPO positive neutrophils in the choroid plexus of (C) controls and (D) 5D C. albicans /Fluconazole animals.

\begin{tabular}{|c|c|c|c|c|c|}
\hline & Control & $\begin{array}{l}\text { 1D } \\
\text { Fluconazole }\end{array}$ & $\begin{array}{l}\text { 3D C. } \\
\text { albicans }\end{array}$ & $\begin{array}{l}\text { 3D C. } \\
\text { albicans and } \\
\text { Fluconazole }\end{array}$ & $\begin{array}{l}\text { 5D } C \text {. } \\
\text { albicans and } \\
\text { Fluconazole }\end{array}$ \\
\hline \multicolumn{6}{|l|}{ Ki67 + cells / mm2 } \\
\hline White matter & $\begin{array}{l}11.2 \\
(7.2)\end{array}$ & $\begin{array}{l}41.6 \\
(38.7)\end{array}$ & $\begin{array}{l}21.9 \\
(16.4)\end{array}$ & $\begin{array}{l}54.7 \\
(71.0)\end{array}$ & $\begin{array}{l}56.0 \\
(31.1)^{*}\end{array}$ \\
\hline $\begin{array}{l}\text { Periventricular white } \\
\text { matter }\end{array}$ & $\begin{array}{l}7.6 \\
(9.1)\end{array}$ & $\begin{array}{l}14.4 \\
(11.2)\end{array}$ & $\begin{array}{l}17.6 \\
(14.2)\end{array}$ & $\begin{array}{l}8.6 \\
(10.7)\end{array}$ & $\begin{array}{l}20.9 \\
(18.9)\end{array}$ \\
\hline Hippocampus & $\begin{array}{l}5.6 \\
(4.2) \\
\end{array}$ & $\begin{array}{l}16.8 \\
(13.6) \\
\end{array}$ & $\begin{array}{l}22.2 \\
(27.5)\end{array}$ & $\begin{array}{l}13.1 \\
(13.0)\end{array}$ & $\begin{array}{l}33.4 \\
(27.0) * \\
\end{array}$ \\
\hline \multicolumn{6}{|l|}{ Caspase $3+$ cells $/$ mm $^{2}$} \\
\hline White matter & $\begin{array}{l}12.8 \\
(13.6)\end{array}$ & $\begin{array}{l}14.1 \\
(11.1)\end{array}$ & $\begin{array}{l}23.7 \\
(33.2)\end{array}$ & $\begin{array}{l}16.9 \\
(6.8)\end{array}$ & $\begin{array}{l}40.2 \\
(22.6)\end{array}$ \\
\hline $\begin{array}{l}\text { Periventricular white } \\
\text { matter }\end{array}$ & $\begin{array}{l}26.1 \\
(11.4)\end{array}$ & $\begin{array}{l}35.4 \\
(24.4)\end{array}$ & $\begin{array}{l}93.4 \\
(110.2)\end{array}$ & $\begin{array}{l}46.4 \\
(29.1)\end{array}$ & $\begin{array}{l}155.1 \\
(22.4)^{*}\end{array}$ \\
\hline Hippocampus & $\begin{array}{l}364.3 \\
(175.3) \\
\end{array}$ & $\begin{array}{l}264.4 \\
(19.6) \\
\end{array}$ & $\begin{array}{l}443.2 \\
(198.8) \\
\end{array}$ & $\begin{array}{l}313.4 \\
(117.0) \\
\end{array}$ & $\begin{array}{l}726.3 \\
(317.9) *\end{array}$ \\
\hline
\end{tabular}

Table 2. Ki67 and Caspase-3 postive cells. Ki67 and Caspase-3 positive cells expressed as mean $( \pm$ SD). $*<0.05$ versus control. 


\section{Discussion}

The main finding of this study is that 3 days of intra-amniotic exposure to C. albicans resulted in increased microglial and astrocyte activation with subsequent focal white matter disturbances in the preterm ovine brain. At this stage, $C$. albicans positive CSF cultures were detected without $C$. albicanspositive immunostainings of the brain. This may indicate that cerebral inflammation and injury in the acute phase after intra-amniotic C. albicans delivery is not caused by invasion of $C$. albicans in the brain parenchyma $[25,26]$. This concept is supported by the absence of neutrophils in the brain parenchyma since neutrophils are known to aggravate disruption of the blood-brain barrier promoting further invasion of $C$. albicans in the brain [25]. However, we cannot rule out the possibility that low titers invaded the brain, since the initial phase of $C$. albicans invasion does not require a disruption of the blood-brain barrier and such low fungal burden of the brain cannot be detected by histology or immunofluorescence [15, 25].

Since C. albicans was not detected in the fetal brain, we considered that severe systemic immune activation, as shown in the present and previous studies [16, 17], was (primarily) responsible for the acute changes observed in the fetal brain 3 days post- $C$. albicans infection of the amniotic cavity for the following reasons: Pro-inflammatory cytokines, such as IL-6, contribute to activation of the endothelium of the blood-brain barrier, which in turn activate adjacent microglia and astrocytes, ultimately resulting in a cerebral inflammatory response [27-31]. In line with this, systemic inflammation is known to be causally involved in the induction of white matter injury - as seen in our model $[32,33]$. In addition, IL-6 was shown to be involved in the inhibition of neurogenesis [28] and the development of hyper-excitable neurological conditions including epilepsy, psychoses, anxiety and autism spectrum disorders in experimental models [34].

Notably, increased circulatory IL-6 concentrations were not always paralleled by positive blood cultures in our study. This finding can be explained by earlier studies $[35,36]$ in which the presence of a pro-inflammatory trigger in the amniotic fluid caused systemic inflammation without migration of 
microorganisms or their components into the fetal circulation. Intra-amniotic $C$. albicans caused a heterogeneous pattern of cerebral immune activation as illustrated by increased IBA-1 immunoreactivity in all C. albicans exposed animals. These regions of overt microgliosis were strongly associated with white matter injury within these specific regions. Moreover, expression of CD68, which is important for the phagocytic activity of macrophages/microglia, was increased at 5 days post exposure, and might play a prominent role the clearance of debris including injured mature oligodendrocytes and myelin [37].

White matter injury in this study was illustrated by reduction of MBP IR at 3 days post $C$. albicans exposure, disrupted myelin texture in all $C$. albicans exposed animals and a deficit of mature oligodendrocytes which was most prominent in the 5 days $C$. albicans/Fluconazole group. This white matter injury is considered to be the result of activated microglia as seen in our study, which produce cytokines, free radicals, nitric oxide, and excitotoxic amino acids, all detrimental for oligodendrocytes and myelin $[7,38]$. In addition, the most prominent loss of mature oligodendrocytes in the 5 days $C$. albicans/Fluzonazole group is most likely the result of clearance of these cells by phagocytizing microglia as previously stated. This deficit was paralleled by replenishment of the oligodendrocyte pool via two mechanisms. First oligodendrocyte progenitors could migrate to the site of injury $[39,40]$. Second increased oligodendrocyte numbers could be the consequence of increased proliferation [39, 41], which would be consistent with our Ki67 data. However, increased Ki67 positive cells do not exclusively identify oligodendrocyte proliferation but could also comprise other cell types. In particular, the increase of Ki67 positive cells could also, in part, account for microglial proliferation, since we observed an increase of microglia within these regions.

Nonetheless, the observed replenishment of oligodendrocytes is considered to be initiated as compensation for the observed reduction of mature oligodendrocytes $[39,41]$. It is tempting to speculate that these progenitors might fail to mature into functional myelin producing oligodendrocyte since this phenotype has previously been reported in experimental models of perinatal stress [42-44] and in human neonates with periventricular leukomalacia [39]. Finally, such oligodendrocyte maturation arrest correlates with motor deficits characteristic for cerebral palsy [38, 44, 45]. In conjunction with white matter injury, grey matter injury is increasingly recognized as a contributor to adverse neurodevelopmental outcome [35]. 
However, in our study no degenerating neurons were observed [23, 22]. Although it is not likely that neurodegeneration is already pertinent in the 5D C. albicans/Fluconazole group, we cannot exclude the possibility that the absence of Fluoro-Jade staining at 5 days post exposure is the result of already degenerated and dead neurons. In addition, cerebral palsy also comprises cognitive impairments $[7,8]$ for which we analyzed the hippocampus in which a similar inflammatory response was observed which is consistent with our previous results $[46,21]$.

We previously reported that intra-amniotic $C$. albicans rapidly colonizes the amniotic fluid, causing inflammation of the fetal skin and lungs, and progresses into a fetal systemic inflammatory response causing fetal death within 5 days after exposure $[16,17]$. In the present study we show that Fluconazole administration eradicated C. albicans from the CSF and reduced systemic inflammation. Importantly, reduction of the fungal burden and inhibition of systemic immune activation following Fluconazole treatment was not accompanied by attenuation of cerebral inflammation and injury. Multiple studies have demonstrated that cerebral inflammation acquired during early fetal development continues postnatally and might even persist into adulthood [47, 48]. Therefore, we consider that the observed cerebral inflammatory response following intra-amniotic $C$. albicans exposure is a persistent reaction that is initiated by peripheral immune activation. Although Fluconazole treatment did not protect against structural cerebral injury in this acute phase, the observed inhibition of systemic immune activation might be clinically relevant, since prolonged and aberrant systemic immune activation are known to induce blood-brain barrier disruption, thereby facilitating cerebral invasion of $C$. albicans and substantially aggravating cerebral injury resulting in increased morbidity and mortality [26]. Collectively, previous and current findings suggest that timing of infection and the start of antifungal treatment regimen are important in the initiation, maintenance, and possible resolution of neuroinflammation and structural injury in the fetal brain. The current study provides important insight in the sequelae of events that contribute to induction of adverse outcomes of the premature brain following intraamniotic C. albicans infection. In addition, this study points out that Fluconazole treatment should be started immediately after intra-amniotic exposure to $C$. albicans considering the narrow therapeutic time window to reduce morbidity and mortality. Therefore, further research should focus on timing, frequency and dosing of antifungals in the presence or absence of immunomodulatory treatment [49]. 
Fluconazole treatment was not associated with adverse effects in our study, which is in line with the findings of Maneenil and colleagues in the same model [16] and studies demonstrating that teratogenic effects of Fluconazole are exclusively seen when used in the first trimester at higher doses and after multiple gifts [50]. The administered Fluconazole dose in this study mimics the amount used in systemic neonatal candidiasis in which no adverse long-term brain pathology and behavioral deficits are currently described [50]. In addition, clinical in utero administration of Fluconazole prolonged pregnancy in two cases of intra-amniotic $C$. albicans infection without adverse treatment effects on the fetus [13].

We note that our study has several limitations. We only tested one dose and frequency of Fluconazole administration. In addition, we were not able to correlate histological changes to functional neurological outcomes. However, several studies previously established an association between endotoxin-induced chorioamnionitis and functional EEG changes [51, 52, 43].

\section{Conclusions}

We have shown that intra-amniotic exposure to $C$. albicans results in an acute systemic and neuroinflammatory response with concomitant white matter injury. Although systemic immune activation was significantly inhibited following Fluconazole treatment, modulation of the cerebral inflammatory response and prevention of concomitant white matter injury was not found.

This study forms the essential basis for follow up studies in which the timing, frequency and dosing of antifungal treatment must be explored and alternative/additional neuroprotective treatment options including immunomodulatory interventions can be tested. 


\section{References}

1. Agrawal V, Hirsch E. Intrauterine infection and preterm labor. Seminars in fetal \& neonatal medicine. 2012;17(1):12-9.

2. Goldenberg RL, Culhane JF, Iams JD, Romero R. Epidemiology and causes of preterm birth. Lancet. 2008;371(9606):75-84.

3. Goldenberg RL, Hauth JC, Andrews WW. Intrauterine infection and preterm delivery. The New England journal of medicine. 2000;342(20):1500-7.

4. Galinsky R, Polglase GR, Hooper SB, Black MJ, Moss TJ. The consequences of chorioamnionitis: preterm birth and effects on development. Journal of pregnancy. 2013;2013:412831.

5. Gantert M, Been JV, Gavilanes AW, Garnier Y, Zimmermann LJ, Kramer BW. Chorioamnionitis: a multiorgan disease of the fetus? Journal of perinatology : official journal of the California Perinatal Association. 2010;30 Suppl:S21-30.

6. Malaeb S, Dammann O. Fetal inflammatory response and brain injury in the preterm newborn. Journal of child neurology. 2009;24(9):1119-26.

7. Kuypers E, Ophelders D, Jellema RK, Kunzmann S, Gavilanes AW, Kramer BW. White matter injury following fetal inflammatory response syndrome induced by chorioamnionitis and fetal sepsis: lessons from experimental ovine models. Early human development. 2012;88(12):931-6.

8. Wu YW. Systematic review of chorioamnionitis and cerebral palsy. Mental retardation and developmental disabilities research reviews. 2002;8(1):25-9.

9. Chau V, McFadden DE, Poskitt KJ, Miller SP. Chorioamnionitis in the pathogenesis of brain injury in preterm infants. Clinics in perinatology. 2014;41(1):83-103.

10. Yoon BH, Romero R, Park JS, Kim CJ, Kim SH, Choi JH et al. Fetal exposure to an intraamniotic inflammation and the development of cerebral palsy at the age of three years. American journal of obstetrics and gynecology. 2000;182(3):675-81.

11. DiGiulio DB, Romero R, Amogan HP, Kusanovic JP, Bik EM, Gotsch F et al. Microbial prevalence, diversity and abundance in amniotic fluid during preterm labor: a molecular and culture-based investigation. PloS one. 2008;3(8):e3056.

12. Payne MS, Bayatibojakhi S. Exploring preterm birth as a polymicrobial disease: an overview of the uterine microbiome. Frontiers in immunology. 2014;5:595.

13. Bean LM, Jackson JR, Dobak WJ, Beiswenger TR, Thorp JA. Intra-amniotic fluconazole therapy for Candida albicans intra-amniotic infection. Obstetrics and gynecology. 
2013;121(2 Pt 2 Suppl 1):452-4.

14. Meizoso T, Rivera T, Fernandez-Acenero MJ, Mestre MJ, Garrido M, Garaulet C. Intrauterine candidiasis: report of four cases. Archives of gynecology and obstetrics. 2008;278(2):173-6.

15. Tsai NY, Laforce-Nesbitt SS, Tucker R, Bliss JM. A murine model for disseminated candidiasis in neonates. Pediatric research. 2011;69(3):189-93.

16. Maneenil G, Payne MS, Kannan PS, Kallapur SG, Kramer BW, Newnham JP et al. Fluconazole Treatment of Intrauterine Candida albicans Infection in Fetal Sheep. Pediatric research. 2015.

17. Payne MS, Kemp MW, Kallapur SG, Kannan PS, Saito M, Miura Y et al. Intrauterine Candida albicans infection elicits severe inflammation in fetal sheep. Pediatric research. 2014;75(6):716-22.

18. Moudgal VV, Sobel JD. Antifungal drugs in pregnancy: a review. Expert opinion on drug safety. 2003;2(5):475-83.

19. Collins JJ, Kallapur SG, Knox CL, Kemp MW, Kuypers E, Zimmermann LJ et al. Repeated intrauterine exposures to inflammatory stimuli attenuated transforming growth factor-beta signaling in the ovine fetal lung. Neonatology. 2013;104(1):49-55.

20. Back SA, Riddle A, Hohimer AR. Role of instrumented fetal sheep preparations in defining the pathogenesis of human periventricular white-matter injury. Journal of child neurology. 2006;21(7):582-9.

21. Gavilanes AW, Strackx E, Kramer BW, Gantert M, Van den Hove D, Steinbusch H et al. Chorioamnionitis induced by intraamniotic lipopolysaccharide resulted in an interval-dependent increase in central nervous system injury in the fetal sheep. American journal of obstetrics and gynecology. 2009;200(4):437 e1-8.

22. Rocha E, Hammond R, Richardson B. Necrotic cell injury in the preterm and near-term ovine fetal brain after intermittent umbilical cord occlusion. American journal of obstetrics and gynecology. 2004;191(2):488-96.

23. Schmued LC, Stowers CC, Scallet AC, Xu L. Fluoro-Jade C results in ultra high resolution and contrast labeling of degenerating neurons. Brain research. 2005;1035(1):24-31.

24. Perego C, Fumagalli S, De Simoni M-G. Temporal pattern of expression and colocalization of microglia/macrophage phenotype markers following brain ischemic injury in mice. Journal of neuroinflammation. 2011;8(1):174.

25. Navarathna DH, Munasinghe J, Lizak MJ, Nayak D, McGavern DB, Roberts DD. MRI confirms loss of blood-brain barrier integrity in a mouse model of disseminated candidiasis. NMR in biomedicine. 2013;26(9):1125-34.

26. Lionakis MS, Lim JK, Lee CC, Murphy PM. Organ-specific innate immune responses in a mouse model of invasive candidiasis. Journal of innate immunity. 2011;3(2):180-99.

27. Rivest $S$. Molecular insights on the cerebral innate immune system. Brain, behavior, and immunity. 2003;17(1):13-9.

28. Deverman BE, Patterson PH. Cytokines and CNS development. Neuron. 2009;64(1):61- 
78. 29. Dammann O, O'Shea TM. Cytokines and perinatal brain damage. Clinics in perinatology. 2008;35(4):643-63, v.

29. Dammann O, O'Shea TM. Cytokines and perinatal brain damage. Clinics in perinatology. 2008;35(4):643-63, v.

30. Hoogland IC, Houbolt C, van Westerloo DJ, van Gool WA, van de Beek D. Systemic inflammation and microglial activation: systematic review of animal experiments. J Neuroinflammation. 2015;12:114.

31. Biesmans S, Meert TF, Bouwknecht JA, Acton PD, Davoodi N, De Haes P et al. Systemic immune activation leads to neuroinflammation and sickness behavior in mice. Mediators of inflammation. 2013;2013:271359.

32. Gomez R, Romero R, Ghezzi F, Yoon BH, Mazor M, Berry SM. The fetal inflammatory response syndrome. American journal of obstetrics and gynecology. 1998;179(1):194202.

33. Rees S, Inder T. Fetal and neonatal origins of altered brain development. Early human development. 2005;81(9):753-61.

34. Atzori M, Garcia-Oscos F, Mendez JA. Role of IL-6 in the etiology of hyperexcitable neuropsychiatric conditions: experimental evidence and therapeutic implications. Future medicinal chemistry. 2012;4(17):2177-92.

35. Wolfs TG, Kramer BW, Thuijls G, Kemp MW, Saito M, Willems MG et al. Chorioamnionitis-induced fetal gut injury is mediated by direct gut exposure of inflammatory mediators or by lung inflammation. American journal of physiology Gastrointestinal and liver physiology. 2014;306(5):G382-93.

36. Kemp MW, Kannan PS, Saito M, Newnham JP, Cox T, Jobe AH et al. Selective exposure of the fetal lung and skin/amnion (but not gastro-intestinal tract) to LPS elicits acute systemic inflammation in fetal sheep. PloS one. 2013;8(5):e63355.

37. Perego C, Fumagalli S, DeSimoni MG. Temporal pattern of expression and colocalization of microglia/macrophage phenotype markers following brain ischemic injury in mice. Journal of neuroinflammation. 2011;8:174.

38. Volpe JJ. Brain injury in premature infants: a complex amalgam of destructive and developmental disturbances. The Lancet Neurology. 2009;8(1):110-24.

39. Billiards SS, Haynes RL, Folkerth RD, Borenstein NS, Trachtenberg FL, Rowitch DH et al. Myelin abnormalities without oligodendrocyte loss in periventricular leukomalacia. Brain pathology. 2008;18(2):153-63.

40. Bonestroo HJ, Heijnen CJ, Groenendaal F, van Bel F, Nijboer CH. Development of cerebral gray and white matter injury and cerebral inflammation over time after inflammatory perinatal asphyxia. Developmental neuroscience. 2015;37(1):78-94.

41. Filipovic R, Zecevic N. Lipopolysaccharide affects Golli expression and promotes proliferation of oligodendrocyte progenitors. Glia. 2005;49(4):457-66.

42. Back SA, Miller SP. Brain injury in premature neonates: A primary cerebral dysmaturation disorder? Annals of neurology. 2014;75(4):469-86. 
43. Dean JM, van de Looij Y, Sizonenko SV, Lodygensky GA, Lazeyras F, Bolouri $\mathrm{H}$ et al. Delayed cortical impairment following lipopolysaccharide exposure in preterm fetal sheep. Annals of neurology. 2011;70(5):846-56.

44. Segovia KN, McClure M, Moravec M, Luo NL, Wan Y, Gong X et al. Arrested oligodendrocyte lineage maturation in chronic perinatal white matter injury. Annals of neurology. 2008;63(4):520-30.

45. Buser JR, Maire J, Riddle A, Gong X, Nguyen T, Nelson K et al. Arrested preoligodendrocyte maturation contributes to myelination failure in premature infants. Annals of neurology. 2012;71(1):93-109.

46. Kuypers E, Jellema RK, Ophelders DR, Dudink J, Nikiforou M, Wolfs TG et al. Effects of intra-amniotic lipopolysaccharide and maternal betamethasone on brain inflammation in fetal sheep. PloS one. 2013;8(12):e81644.

47. Fleiss B, Gressens P. Tertiary mechanisms of brain damage: a new hope for treatment of cerebral palsy? The Lancet Neurology. 2012;11(6):556-66.

48. Dammann O. Persistent neuro-inflammation in cerebral palsy: a therapeutic window of opportunity? Acta paediatrica. 2007;96(1):6-7.

49. Wolfberg AJ, Dammann O, Gressens P. Anti-inflammatory and immunomodulatory strategies to protect the perinatal brain. Seminars in fetal \& neonatal medicine. 2007;12(4):296-302.

50. Alsaad AM, Kaplan YC, Koren G. Exposure to fluconazole and risk of congenital malformations in the offspring: A systematic review and meta-analysis. Reproductive toxicology. 2015;52:78-82.

51. Keogh MJ, Bennet L, Drury PP, Booth LC, Mathai S, Naylor AS et al. Subclinical exposure to low-dose endotoxin impairs EEG maturation in preterm fetal sheep. American journal of physiology Regulatory, integrative and comparative physiology. 2012;303(3):R270-8.

52. Gavilanes AW, Gantert M, Strackx E, Zimmermann LJ, Seeldrayers S, Vles JS et al. Increased EEG delta frequency corresponds to chorioamnionitis-related brain injury. Frontiers in bioscience. 2010;2:432-8. 


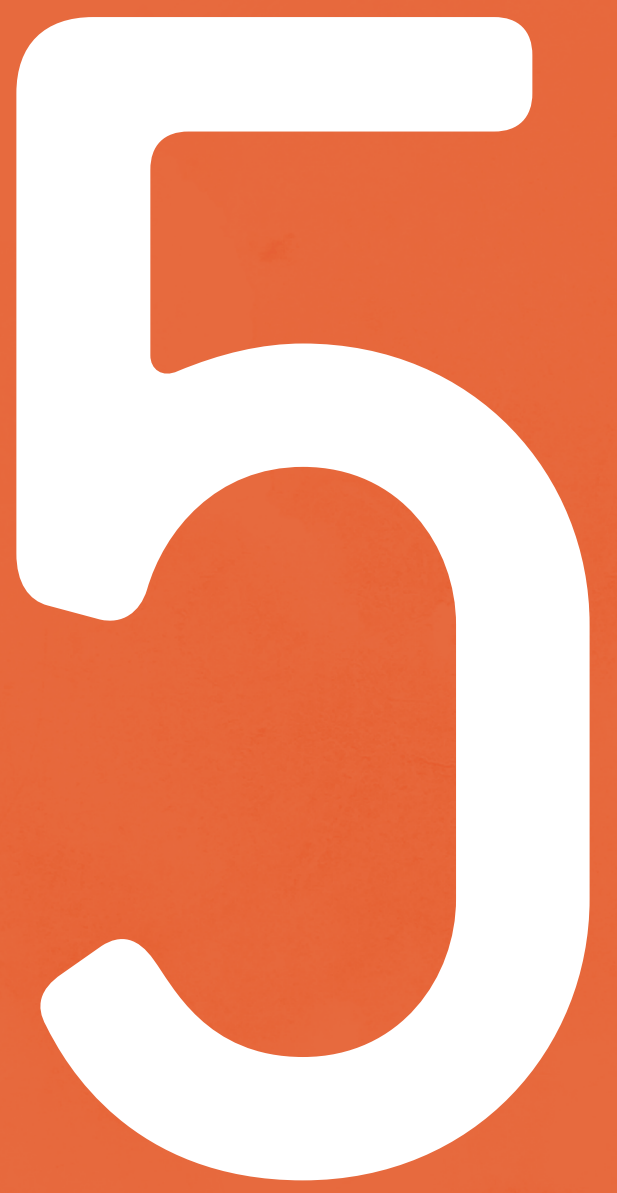

Systemic Multipotent Adult Progenitor Cells protect the cerebellum after asphyxia in fetal sheep. 



\section{Abstract}

\section{Background}

Involvement of the cerebellum in the pathophysiology of hypoxic-ischemic encephalopathy (HIE) in preterm infants is increasingly recognized. We aimed to assess the neuroprotective potential of intravenously administered Multipotent Adult Progenitor Cells (MAPC®) in the preterm cerebellum.

\section{Methods}

Instrumented preterm ovine fetuses were subjected to transient global hypoxia-ischemia (HI) by 25 minutes of umbilical cord occlusion at 0.7 of gestation. After reperfusion, two doses of MAPC cells were administered intravenously.

\section{Results}

Global HI caused marked cortical injury in the cerebellum, histologically indicated by disruption of cortical strata, impeded Purkinje cell development and decreased dendritic arborisation. Furthermore, global HI induced histopathological microgliosis, hypomyelination and disruption of white matter organization. MAPC cell treatment significantly prevented cortical injury and region-specifically attenuated white matter injury in the cerebellum following global HI. Diffusion tensor imaging (DTI) detected HI-induced injury and MAPC cell neuroprotection in the preterm cerebellum.

\section{Conclusion}

This study has demonstrated in a preclinical large animal model that early systemic MAPC therapy improved structural injury of the preterm cerebellum following global HI. Microstructural improvement was detectable with DTI. These findings support the potential of MAPC cell therapy for the treatment of HIE and the added clinical value of DTI for the detection of cerebellar injury and the evaluation of cell-based therapy. 


\section{Significance statement}

Cerebellar injury after asphyxia was reduced with systemic multipotent adult progenitor cells (MAPC) therapy. These findings in the cerebellum essentially extended our previous experimental work showing that MAPC cells induced structural and functional neuroprotection after asphyxia in the ovine cerebrum. MAPC cells are a clinical-grade product with extensively proven safety and a recent clinical trial in adult stroke patients showed that MAPC cells improved functional outcomes after 1-year followup. Our work in a relevant large animal model with clinical-grade stem cells greatly improve the potential for translation into neonatal medicine. 


\section{Introduction}

Hypoxic-ischemic encephalopathy (HIE) is an important cause of mortality and morbidity in preterm neonates [1]. Global hypoxic-ischemic encephalopathy in preterm infants affects individual brain regions differently [1, 2]. HIE was recognized primarily as a white matter disease of the cerebrum, leaving cerebellar involvement underappreciated in the clinical setting [2]. However, previous findings demonstrate that cerebellar injury plays an important role in the high prevalence of non-motor deficits (i.e. cognition, learning and behavior) in survivors of prematurity [3].

During the third trimester of pregnancy the cerebellum exhibits a rapid increase in growth and development [2]. Any disturbance of cerebellar growth e.g. by hypoxiaischemia or inflammation, may lead to irreversible aberrations of the cerebellar integrity [2, 4]. Moreover, cerebellar lesions in the developing brain can cause or aggravate contra-lateral supratentorial deficits (cerebellocerebral diaschisis) [2].

Until recently, acute cerebellar injuries were rarely detected with conventional clinical neuroimaging, such as T1- and T2-weighted magnetic resonance imaging (MRI) [5]. Clinical imaging studies using new advanced MRI modalities, including diffusion tensor imaging (DTI), however showed that cerebellar injury in premature survivors with neonatal encephalopathy is common and associated with adverse clinical outcome [6]. Moreover, disturbed cerebellar development in prematurely born children can still be detected at school age/adolescence with advanced neuroimaging [7].

Therefore, protection of the cerebellum following preterm birth and/or $\mathrm{HI}$ is clinically highly relevant and cell-based therapy has proven to be a promising treatment to improve neurodevelopmental outcomes in preterm infants [8]. We have previously shown that intravenous administration of mesenchymal stem cells (MSC) and multipotent adult progenitor cells (MAPC) protect cerebral structure and function in a fetal sheep model of HIE [9, 10]. In this model of preterm brain injury, we demonstrated that the injurious effects of global hypoxia-ischemia and therapeutic effects of intravenous stem cell therapy could be readily detected in the cerebral white matter using DTI [9]. 
The primary aim of this study was therefore to assess the neuroprotective potential of MAPC cellsin the cerebellum following global hypoxia-ischemia (HI). We hypothesized that MAPC cell therapy would reduce structural injury in the preterm cerebellum following global $\mathrm{HI}$. To test this hypothesis, 0.7 gestation instrumented fetal sheep were exposed to global hypoxia-ischemia by umbilical cord occlusion and subsequently treated with intravenously delivered MAPC cells. Cerebellar injury was assessed by histopathology and diffusion weighted MRI. 


\section{Material and methods}

\section{Study approval}

All animal procedures were performed in accordance with the guidelines and approval of the institutional Animal Ethics Research committee of Maastricht University, The Netherlands.

\section{Multi-potent Adult Progenitor Cells}

Multipotent adult progenitor cells (MAPC) are a plastic adherent bonemarrow derived population of adult progenitor cells with long-term culture expansion and potency [11, 12]. MultiStem ${ }^{\circledR}$, the clinical grade MAPC product, is currently being tested for neuroprotection in an international phase III trial of adult stroke (clinicaltrial.gov NCT03545607) [13]. Differentiation capacity, functional characteristics and genomic and epigenetic stability of the MAPC cells were confirmed as previously described [11, 12]. MAPC cells were expanded under Good Manufacturing Practice (GMP) as previously described [14] and stored in liquid nitrogen. Before administration, the MAPC cells were thawed and washed twice and suspended in PBS at a concentration of $10 \times 106$ cells $/ \mathrm{ml}$. The fetuses randomized to MAPC cell treatment received two intravenously delivered doses of $10 \times 106$ cells in $1 \mathrm{ml}$ PBS.

\section{Experimental design}

Fetuses of time-mated Texel ewes were instrumented as previously described [9]. The outcome of the cerebrum of these animals has been previously reported [10]. We assessed the cerebellum of twenty-three $(n=23)$ singleton fetuses (both sexes), which had been randomly assigned to one of the four following experimental groups: (1) sham umbilical cord occlusion, saline treatment (sham-SAL; $\mathrm{n}=8$ ), (2) sham umbilical cord occlusion, MAPC treatment (sham-MAPC; $\mathrm{n}=3$ ), (3) umbilical cord occlusion, saline treatment (HI-SAL or HI; $\mathrm{n}=7$ ) and (4) umbilical cord occlusion, MAPC treatment (HI-MAPC; $n=5$ ). Power analysis indicated that with a sample size of $n=5$ in the HI-MAPC group significant treatment effects could be detected in the cerebellum, which was based on previous reported MAPC- 
mediated white matter protection in the cerebrum [10]. Previous clinical [13] and experimental [10,15-17] work has extensively shown safety of MAPC cells under control conditions. Therefore, a smaller sham-MAPC group of $n=3$ was considered acceptable to exclude MAPCinduced changes in the cerebellum under sham conditions.

Fetal instrumentation was performed at 102 days of gestation (experimental day -4). At 106 days of gestation (experimental day 0) fetuses were subjected to 25 minutes of umbilical cord occlusion (UCO) or sham conditions. At the time of UCO, brain maturation is broadly equivalent to 30 weeks of human gestation which coincides with the high-risk window of brain injury in preterm infants [18]. The dynamics of physiological parameters during UCO were previously reported [10]. Fetuses received two doses of MAPC cells or an equal volume of the vehicle (1 hour and 4 days following UCO). Randomization, (sham) UCO and tissue sampling was performed by investigators who were blinded for treatment allocation.

\section{Brain sampling}

After a reperfusion period of 7 days, animals were sacrificed at 113 days of gestation with intravenous pentobarbitone $(200 \mathrm{mg} / \mathrm{kg})$. Brains were collected and the right hemisphere was submersion fixated in $4 \%$ paraformaldehyde (PFA) at $4^{\circ}$ Celsius for 3 months.

\section{Magnetic Resonance Imaging}

To wash out the PFA, forty-eight hours prior to MRI imaging the right hemisphere was washed with PBS and stored in PBS containing 1\% sodium azide. DTI images were acquired using an echo planar imaging (EPI) sequence with diffusion gradients $(\mathrm{b}=4000 \mathrm{~s} / \mathrm{mm} 2)$ applied in 66 non-collinear directions and 6 B0 measurements. An average of 60 slices was recorded within 36 minutes using a repetition time $(\mathrm{TR})=500 \mathrm{~ms}$ and echo time $(\mathrm{TE})=75 \mathrm{~ms}$. Isovolumetric voxel size was $0.5 \mathrm{~mm} 3$. The FOV was 30x60x60 $\mathrm{mm}$ and scan matrix size $60 \times 120 \times 60 \mathrm{~mm}$.

Diffusion weighted images were processed using ExploreDTI version 4.8.6 running in Matlab (R2015, The MathWork Inc., Natick, MA) [19]. To optimize image quality, all datasets were corrected for motion, EPI-induced distortions and Eddy currents [19, 20]. Diffusion tensors were calculated robustly using the REKINDLE approach to exclude motion-induced outliers prior to tensor estimation [21]. For each dataset, color-coded FA 
maps were spatially aligned with the T2 image, and visualized as a semitransparent overlay on the T2 maps. This visualization approach improved localization of the desired anatomical regions of interest. After region of interest (ROI) placement in the cerebellum (white matter and cortex) FA values were obtained for each ROI in Explore DTI. Manual delineation of ROIs was performed by a neonatologist with expertise in DTI analysis who was blinded for the treatment allocation.

\section{Histology and immunohistochemistry}

The right hemisphere of the cerebellum was embedded in paraffin and serial sagittal sections $(4 \mu \mathrm{m})$ were cut with a Leica RM2235 microtome (Leica, Rijswijk, The Netherlands) [22]. At the intersection of the cerebellar peduncles, Nissl and Luxol Fast Blue (LFB) stainings were performed for morphological and anatomical analysis. Immunohistochemical analysis were performed on three sections per animal (every 10th consecutive section). Adjacent sections were stained with an anti-ionized calcium binding adaptor molecule 1 (IBA-1) antibody (019-19741, Wako Pure Chemical Industries) for microglia, a glial fibrillary acidic protein (GFAP) antibody (ZO334, Dako) for astroglia, a myelin basic protein (MBP) antibody (MAB386, Milipore) for mature oligodendrocytes and myelin, a calbindin antibody (CB-38a, Swant) for Purkinje cells (PC) and a cleaved caspase-3 antibody (\#9664, Cell signaling) for apoptotic cell death.

Endogenous peroxidase activity was inactivated with $0.3 \% \mathrm{H} 2 \mathrm{O} 2$ treatment. Antigen retrieval was performed by microwave boiling of tissue sections in citrate buffer ( $\mathrm{pH}$ 6.0) [22]. Nonspecific binding was blocked by incubation with bovine, goat or donkey serum in PBS. Sections were incubated overnight at $4^{\circ} \mathrm{C}$ with the diluted primary antibody (IBA-1, GFAP, MBP \& Caspase-3 1:1000; calbindin 1:6000). The following day sections were incubated with the specific secondary antibody and staining was enhanced with a Vectastain $\mathrm{ABC}$ peroxidase Elite kit (Vector Laboratories Inc, Burlingame, $\mathrm{CA}$ ) and (nickel) 3,3'-diaminobenzidine staining.

\section{Qualitative analysis}

Nissl stained sections were analyzed by a neuropathologist with expertise in the field of neonatal brain injuries and two independent researchers who were blinded for the experimental set up. Sections were assessed for structural and inflammatory changes including the presence of gliosis, hemorrhages and structural damage, including cyst formation. LFB sections were evaluated for myelin structure and myelin abnormalities. 


\section{Quantitative analysis of Nissl staining}

The thickness of cerebellar strata was measured on Nissl stained sections. The molecular layer (ML) and external granular layer (EGL) thickness were determined in Image $1.49 \mathrm{v}$ (U. S. National Institutes of Health, Bethesda, Maryland, USA) using 20x images of lobule I - X from Nissl stained cerebellar sections. The thickness of the ML was defined as the distance between the top of the Purkinje cell (PC) soma and the internal border of the EGL. EGL thickness was determined relative to the ML. Measurements were performed in duplicate by two independent researchers on 20 images per animal. One image was taken of the crest and one of the insert of each lobule (lobule I-X) (Figure 1), resulting in 20 images per animal.

\section{Quantitative analysis of immunohistochemical stained sections}

Immunohistochemical stainings were analyzed using a light microscope (Leica DM2000) equipped with Leica QWin Pro version 3.4.0 software (Leica Microsystems, Mannheim, Germany). Inflammatory changes were assessed by IBA-1 immunoreactivity (IR) and white matter alterations were assessed by MBP IR in the anterior cerebellum (lobule III) and posterior cerebellum (lobule IX) (Figure 1). Region of interests were both the white matter and cortex of the cerebellum; as illustrated in Figure 1 the cortical region of interest comprised all three cortical layers i.e. IGL, ML and EGL, whereas the white matter was marked from the beginning of the lobe till the boundary of the IGL. Area fractions were determined using a standard threshold to detect positive staining with Leica QWin Pro V 3.5.1 software (Leica, Rijswijk, The Netherlands). In the same regions, caspase positive cells were counted and expressed as positive cells / $\mathrm{mm} 2$.

PC density was quantified by counting all cell somas positive for calbindin that intersect with a horizontal line of known length and then dividing the number of cells by the length of the line. Counting was performed in lobule III and IX in four random fields of views per lobule.

The areal densities of GFAP+ cells and in particular GFAP+ Bergmann glia that are only located in the $\mathrm{ML}$, were determined by measuring area fractions of GFAP IR in the cerebellar white matter and IGL and ML of the cerebellar cortex using Leica QWin Pro V 3.5.1 software (Leica, Rijswijk, The Netherlands). In addition to area fractions, the linear density of the Bergmann glial fibers (GFAP+ fibers) in the ML was determined using ImageJ. Perpendicular to the Bergmann glial fibers a horizontal line was drawn and fibers that intersected this line were counted in three random fields of view in lobe III and three in 
lobe IX ( 6 fields of view per animal). Linear density was calculated by dividing the number of intersecting fibers by the length of the line and displayed in intersections $/ \mathrm{mm}$.

\section{Statistical analysis}

All values are shown as means with $95 \%$ confidence interval or standard deviations with significance level at $\mathrm{p}<0.05$. Comparison between different experimental groups was performed with analysis of variance (ANOVA) and Bonferroni post-hoc testing. In case data were positively skewed, log-transformation was applied to obtain normal distributed data or non-parametric Kruskal-Wallis test was performed with Dunn's post-hoc testing. Statistical analysis was performed with IBM SPSS Statistics Version 22.0 (IBM Corp., Armonk, NY, USA; SPSS, RRID: SCR_002865) and GraphPad Prism software (version v5.0; GraphPad Software Inc., La Jolla, CA, USA).

A

B

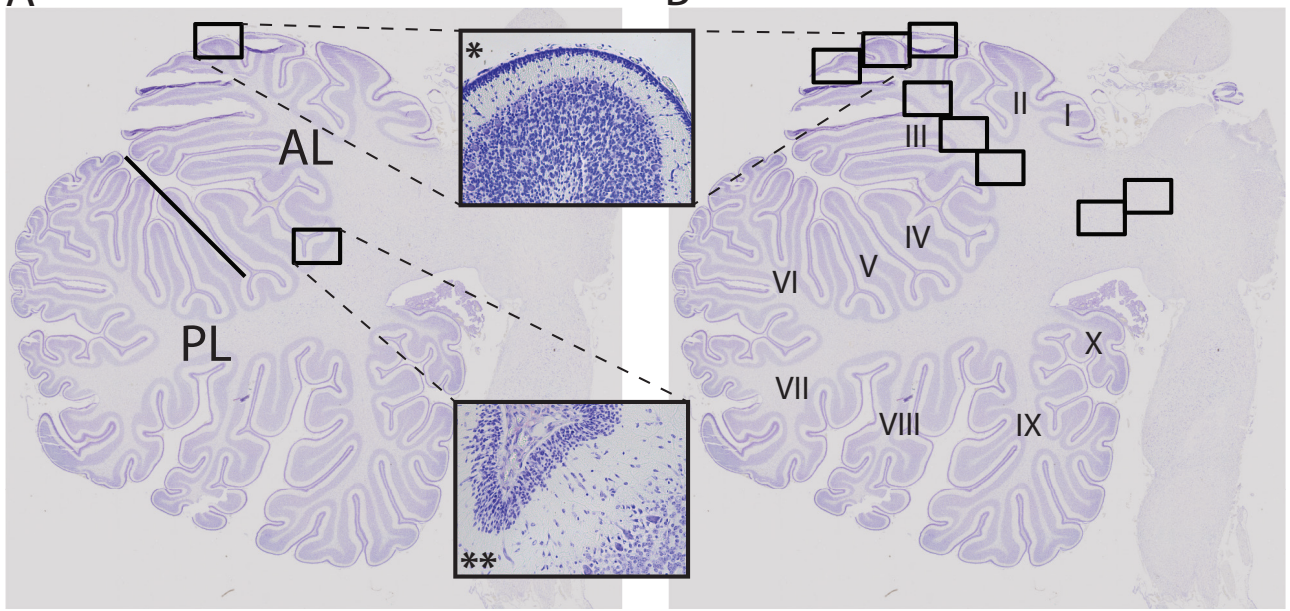

Figure 1. Overview of quantitative analysis. $(A-B)$ Immunohistochemical Nissl staining depicting regions of interest in the cerebellum. (A) Thickness of the external granular layer and molecular layer was measured in the crest $\left.{ }^{*}\right]$ and inserts $\left[{ }^{* *}\right.$ ) of the anterior lobe and the posterior lobe of the cerebellum. (B) Immunohistochemical stainings were analyzed in lobe III and in lobe IX and in the cerebellar peduncles. The black squares indicate the regions of interest in cerebellar white matter, cerebellar cortex and the peduncles. $A L=$ anterior lobe; $P L=$ posterior lobe; ${ }^{*}=$ crest of the lobule; ${ }^{* *}=$ insert of lobule. 


\section{Results}

The structural and functional benefits of MAPC in the cerebrum after global $\mathrm{HI}$ and reproducibility of physiological parameters of the animals in this study have been previously reported [10]. One animal in the HI-MAPC group was excluded from analysis because of maternal postoperative complications necessitating early delivery (day 3 post UCO) of the fetus before the end of the experiment (day 7 post UCO). In the cerebellum, no significant differences in histopathological and neuroimaging outcome parameters were found between the sham-SAL and sham-MAPC group which is consistent with previous experimental studies [10, 15, 17]. Therefore, sham-SAL and sham-MAPC groups were pooled and presented as one control (CTR) group.

\section{MAPC cell therapy prevented histopathological alterations in the cerebellar cortex after global HI}

Global HI significantly induced a decrease of the ML thickness (CTR vs. HI $p<0.001$ ) and not an increase of the EGL thickness (CTR vs. HI $p=0.161$ ). This resulted in a significant increase of the EGL/ML-ratio (CTR vs. HI $\mathrm{p}=0.007$ ) (Figure 2). Following HI, cortical layers were morphologically less defined and cellular density within the EGL and ML was increased following HI compared to control animals (Figure 2).

Purkinje cells (PCs) were analyzed based on cell number and morphology by means of Nissl and calbindin staining. Nissl staining showed that global $\mathrm{HI}$ reduced the number of morphologically normal PCs, increased empty spaces or gaps within the PC layer and increased the number of pyknotic PCs (Figure 2). Pyknotic PCs showed shrunken nuclei with chromatin condensation and reduced or bright pink, acidophilic cytoplasm (Figure 2D, insert). Moreover, there were more PCs present in the internal granular layer (IGL) and cerebellar white matter following HI compared to controls, indicating more ectopic PCs (data not shown).

Global HI-induced loss of PCs was confirmed by a significant (CTR vs. HI $\mathrm{p}<0.001$ ) decrease of calbindin+ cells following HI (Figure 2). In 
addition, dendritic arborisation of calbindin+ cells was reduced following HI compared to controls indicating altered Purkinje cell development or injury (Figure 2). Interestingly, the reduction of dendritic arborisation in the cerebellar cortex was more pronounced in the anterior lobes compared to the posterior lobes of the cerebellum (Figure 3), indicating region-specific neuronal vulnerability in the cerebellum after global HI. Moreover, in the anterior lobe (Figure 3E) markedly more 'gaps' within the PC layer were detected as compared to the posterior lobe (Figure 3G), indicating that loss of PCs was more pronounced in the anterior lobe. Overall, no significant differences (CTR vs. HI p = 0.121) in caspase- $3+$ cells were found following global HI.
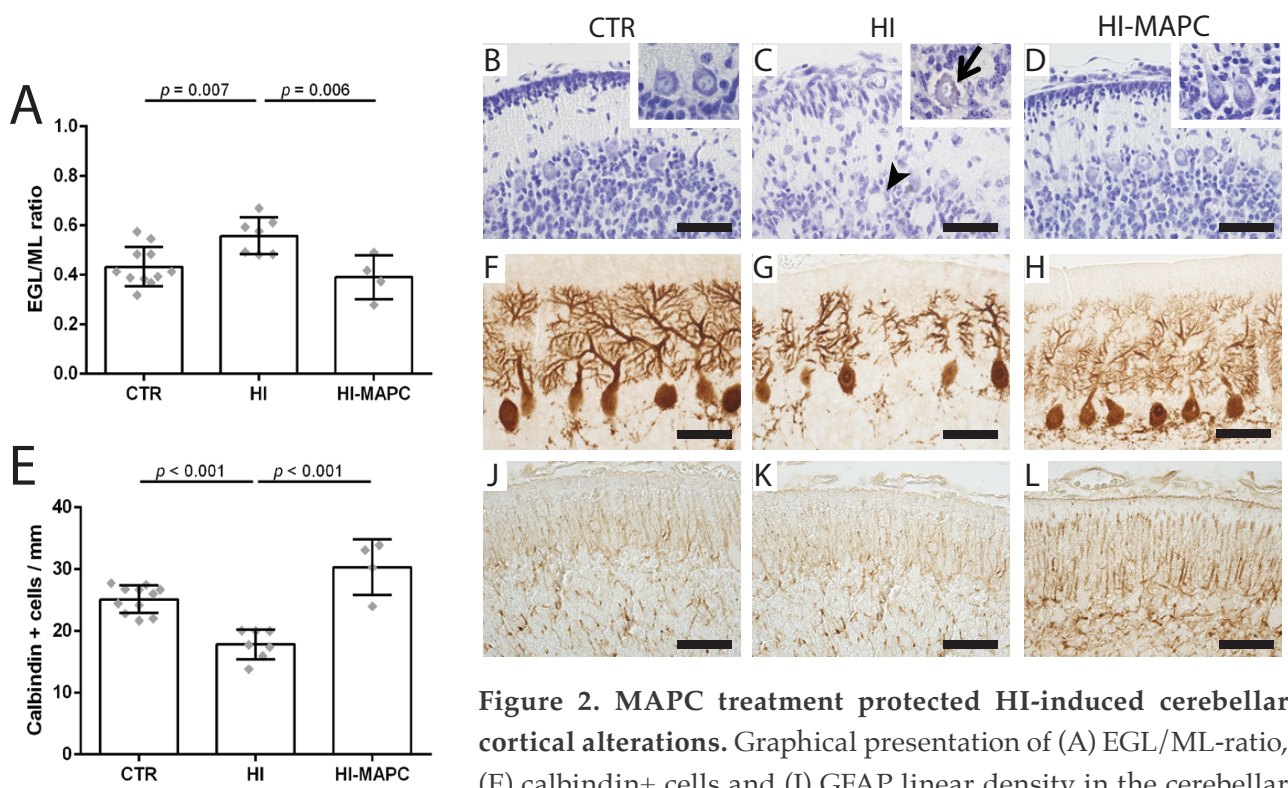

Figure 2. MAPC treatment protected HI-induced cerebellar cortical alterations. Graphical presentation of (A) EGL/ML-ratio, (E) calbindin + cells and (I) GFAP linear density in the cerebellar cortex. Means $\pm 95 \% \mathrm{CI}$ and levels of significance are depicted. Immunohistochemical (B-D) Nissl, (F-H) calbindin and (J-L) GFAP staining in the cerebellar cortex at 400x magnification. Global HI increased the EGL/ML-ratio and decreased the number of calbindin+ cells in the preterm cerebellum. Furthermore, global HI increased the number of pyknotic cells (arrow in insert of panel C), caused more 'gaps' within the PC layer (arrowhead in panel C) and disturbed dendritic arborisation of PCs (panel G). MAPC cell therapy significantly prevented the increase of EGL/ML-ratio and loss of calbindin+ cells after global HI. HI and MAPC treatment did not induced changes in GFAP linear density. CTR = control, $\mathrm{HI}=$ hypoxia-ischemia, $\mathrm{EGL}=$ external granular layer, $\mathrm{ML}=$ molecular layer. Scale $\mathrm{bar}=50 \mu \mathrm{m}$. 
Systemic MAPC cell administration prevented the alterations of cerebellar cortical strata after global $\mathrm{HI}$ as shown by a significantly decreased EGL/ML ratio in the total cerebellum (HI vs. HI-MAPC $\mathrm{p}=0.006$ ). MAPC cell therapy prevented HI-induced loss of Purkinje cells as evidenced by significantly increased number of calbindin+ cells in the total cerebellum (HI vs. HI-MAPC $\mathrm{p}<0.001$ ). Furthermore, the MAPC cells prevented loss of dendritic arborisation after global HI (Figure 2). No significant differences in EGL/ML ratio were found between the control (CTR) and treatment group (HI-MAPC).

\section{ANTERIOR LOBE}
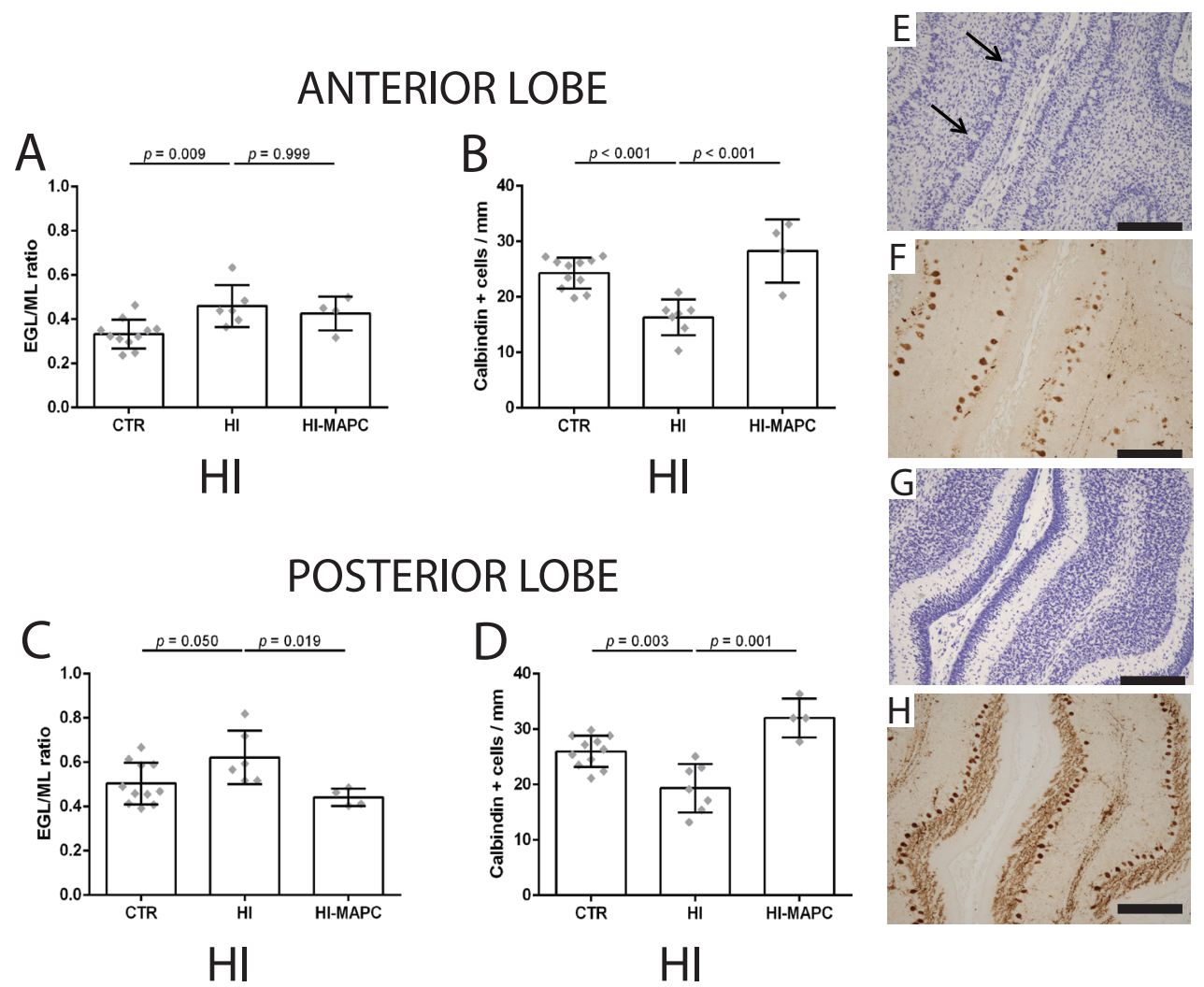

Figure 3. The anterior cerebellum displayed a morphological phenotype distinctly different from the posterior cerebellum following HI.(A-D) Graphical presentation of EGL/ML-ratio and calbindin+ cells in the (A-B) anterior lobe and (C-D) posterior lobe. Means $\pm 95 \% \mathrm{CI}$ and levels of significance are depicted. (E-H) Immunohistochemical (E, G) Nissl and (F, H) calbindin staining in the cerebellar cortex at 100x magnification. Global HI induced more pronounced disruption of cortical layers and loss of calbindin+ cells in the $(E, F)$ anterior lobe compared to the $(G, H)$ posterior lobe of the cerebellum. More 'gaps' (arrows in panel E) within the PC layer of the anterior lobe were seen as compared to the posterior lobe. MAPC cell therapy prevented HI-induced calbindin+ cell loss in both lobes and prevented disruption of cortical strata in the posterior lobe. CTR = control, $\mathrm{HI}=$ hypoxia-ischemia, EGL $=$ external granular layer, $\mathrm{ML}=$ molecular layer. Scale bar $=200 \mu \mathrm{m}$. 


\section{Cortical Bergmann glia were not altered after global HI and MAPC cell therapy}

We assessed linear fiber density of GFAP+ Bergmann glia, which are specialized astrocytes located in the ML of the cerebellar cortex and act as a migration scaffold for granule cells which in turn are important for PC development $[23,24]$. Fiber linear density was not significantly altered following global HI (CTR vs. HI $\mathrm{p}=0.443$ ) (Figure 2I-L). Systemic MAPC cell administration did not alter GFAP+ fiber linear density in the cerebellar cortex following HI (HI vs. HI-MAPC $\mathrm{p}=0.992$ ) (Figure 2I-L).

No significant differences in GFAP IR were found in the cerebellar white matter (CTR vs. HI p = 0.999) and cerebellar cortex GFAP IR (CTR vs. HI $p$ $=0.999)$ after global HI. MAPC cell administration significantly increased GFAP IR in the cerebellar cortex following HI (HI vs. HI-MAPC $\mathrm{p}=0.017$ ) (Figure 2I-L). MAPC cells did not alter GFAP expression in sham conditions.

\section{MAPC cell therapy region-specifically prevented histopathological cerebellar white matter injury after global HI}

White matter injury was assessed by measuring area fraction of Myelin Basic Protein (MBP) IR and quantitative analysis of Luxol Fast Blue (LFB) staining. Global HI significantly reduced MBP IR in the cerebellar white matter (CTR vs HI $p=0.009$ ) indicating hypomyelination (Figure 4A-D). Both MBP (Figure 4B-D) and LFB (Figure 4F-H) staining showed evident disruption and disorganization of myelin sheaths in cerebellar white matter following global HI. No cystic formation or hemorrhages were found in all experimental groups in the cerebellum following global HI.

Systemic MAPC cell administration increased MBP-IR following global HI in the white matter of the cerebellum, however the difference did not reach significance (HI vs. HI-MAPC $\mathrm{p}=0.087$ )(Figure 4A). Sub-analysis indicated that MAPC cell administration did significantly increase MBP-IR in the posterior lobe of the cerebellum after global HI (HI vs. HI-MAPC $\mathrm{p}=0.024)$ (Figure 4E). No significant differences in MBP IR were detected between the control (CTR) and treatment group (HI-MAPC).

\section{MAPC cell therapy did not alter histopathological microgliosis in the preterm cerebellum after global HI}

The cerebellar inflammatory response after global HI was studied by assessing microglial proliferation using ionized calcium binding adaptor, which is a highly specific marker for resting and activated microglia in 
sheep [10]. Global HI significantly increased IBA-1 immunoreactivity in cerebellar white matter (CTR vs. HI $p=0.045)$ and the cerebellar peduncles (CTR vs. HI $\mathrm{p}=0.045)$ indicating marked microglial proliferation in these regions (Figure $4 \mathrm{H}-\mathrm{K}$ ). Within the cerebellar white matter, IBA-IR was increased in the anterior lobes (CTR vs. HI p =0.050) but did not reach significance in the posterior lobes (CTR vs. HI $p=0.117$ ). In the cerebellar cortex, no significant change in IBA-1 IR was detected after global HI (CTR vs. HI p = 0.133) (data not shown). Morphological assessment showed that activated microglia were localized in close association with PCs following HI (Figure 4J insert).

Systemic MAPC cell administration did not alter IBA-1 IR (HI vs HI-MAPC $p=0.999$ ) in the preterm cerebellum following HI (Figure 4). No significant differences in IBA-1 IR were detected between the control (CTR) and treatment group (HI-MAPC).
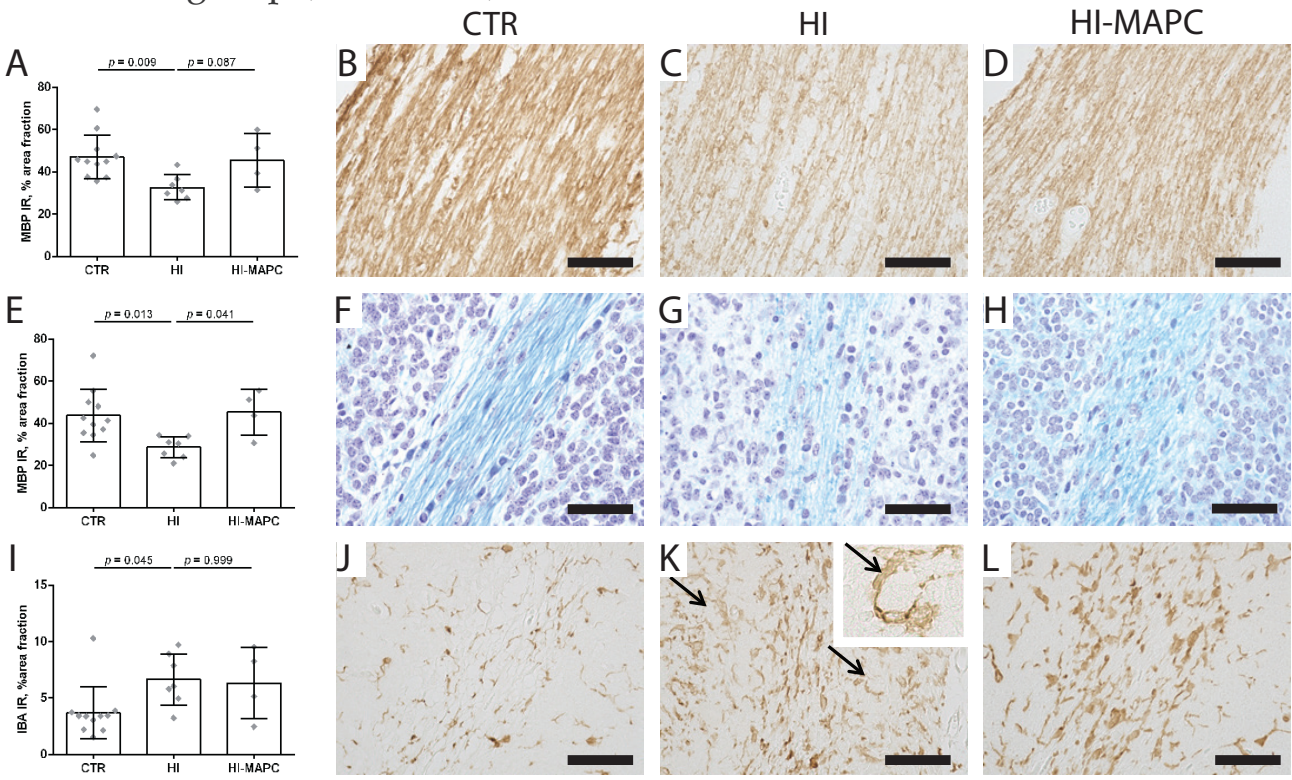

Figure 4. MAPC treatment region-specifically prevented disruption of white matter and hypomyelination without modulating microgliosis in the cerebellar white matter. Graphical presentation of (A, E) MBP IR area fractions of the (A) total cerebellum and (E) posterior lobes and (I) IBA-1 IR area fractions in the total cerebellar white matter. Means $\pm 95 \% \mathrm{CI}$ and levels of significance are depicted. Immunohistochemical (B-D) MBP, (F-H) Luxol fast blue and (J-L) IBA-1 staining in the cerebellar white matter. Global HI caused marked hypomyelination with disorganization of myelin sheets and microglial proliferation in cerebellar white matter. Activated microglia were co-localized with PCs or 'gaps' in the PC layer (arrows in insert of panel K). MAPC cell therapy prevented white matter injury in the preterm cerebellum after global HI. MAPC cell therapy did not alter microglial proliferation in cerebellar white matter. Images are taken at 400x magnification. CTR $=$ control, $\mathrm{HI}=$ hypoxia-ischemia, $\mathrm{IR}=$ immunoreactivity. Scale bar $=50 \mu \mathrm{m}$. 


\section{MAPC cell therapy prevented microstructural changes detected by DTI in the cerebellar cortex after global HI}

FA is a sensitive DTI marker to detect microstructural changes in both white matter and cortex [25]. FA values in the total cerebellum were significantly decreased following global HI (CTR vs. HI p = 0.017) (Figure 5). The decrease of FA values was particularly pronounced in the cerebellar cortex (CTR vs. HI p = 0.007) and did not reach statistical significance in cerebellar white matter (CTR vs. HI p = 0.074). In the cerebellar cortex FA values were positively related to the number of calbindin+ cells in the posterior lobe (Pearson's $r=0.72, p=0.004$ ) and in total cortex (Pearson's $r=0.53, p=$ 0.040 ) and inversely related to linear density of GFAP+ Bergmann glia fibers in the anterior lobe (Pearson's $r=-0.55, p=0.040$ ). In cerebellar white matter FA values were inversely related to IBA-1 IR (Pearson's $r=-0.66$, $\mathrm{p}=0.007$ ) and positively related to MBP IR (Pearson's $\mathrm{r}=0.54, \mathrm{p}=0.036$ ) in the anterior lobe of the cerebellum. No significant differences in mean diffusivity (MD) values in the cerebellum were found following global HI compared to controls (CTR vs. HI p = 0.792).

Systemic MAPC cell administration significantly increased FA values of the total cerebellum (HI vs. HI-MAPC $\mathrm{p}=0.043$ ). The MAPC-induced increase of FA values was particularly pronounced in cerebellar cortex (HI vs. HI-MAPC $\mathrm{p}=0.035)$ and did not reach statistical difference in cerebellar white matter (HI vs. HI-MAPC p=0.626) (Figure 5). No significant differences in FA values were detected between the control (CTR) and treatment group (HI-MAPC).

A

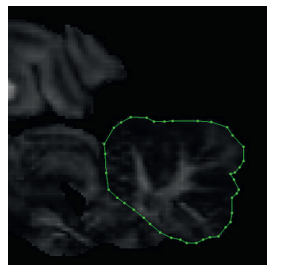

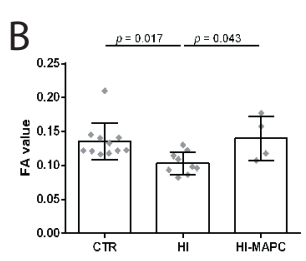
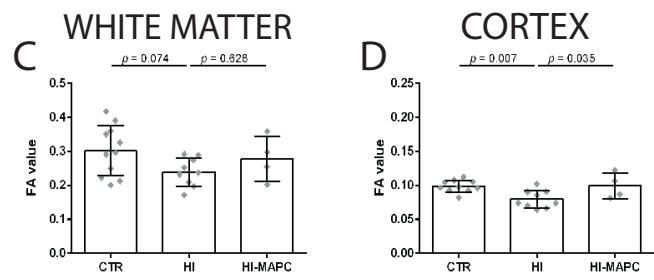

Figure 5. Microstructural changes detected by DTI in the preterm cerebellum following HI and MAPC treatment. (A) Representative FA map depicting the delineation of the cerebellar region of interest. (B-D) Graphical presentation of FA values in the (B) total cerebellum, (C) cerebellar white matter and (D) cerebellar cortex. Means $\pm 95 \% \mathrm{CI}$ and levels of significance are depicted. Global HI significantly decreased FA values in the cerebellum, indicating disruption of cerebellar microstructure. MAPC cell therapy significantly protected the cerebellum against microstructural alterations, particularly in the cerebellar cortex. $\mathrm{CTR}=$ control, $\mathrm{HI}=$ hypoxia-ischemia, $\mathrm{FA}=$ fractional anisotropy. 


\section{Discussion}

Cerebellar injury is increasingly recognized as a contributor to hypoxicischemic encephalopathy (HIE) in preterm infants. Nowadays, cell-based therapy is considered a promising strategy for the treatment of HI-induced white matter injury in the neonatal brain [26]. This is the first study in a preclinical large animal model demonstrating that systemic stem cell therapy may have the potential to protect the preterm cerebellum after global HI.

We found in our model that global HI induced a pattern of injury with disrupted cortical strata, white matter injury and microgliosis in the preterm ovine cerebellum. More precisely, we showed that global HI increased the EGL/ML-ratio, which could not be explained by altered apoptotic cell death within this layer. The latter finding might be explained by the timing of analysis and/or involvement of other mechanisms of cell death (i.e. necrosis or autophagy). The altered EGL/ML ratio may also be the result of impeded migration of the granular cells from the EGL to the IGL reflecting cerebellar underdevelopment following global HI [2]. In line, global HI increased the number of ectopic PCs, which is indicative for a migration arrest of PCs from the ependymal zone to the cerebellar cortex [4]. The disruption of cortical strata within this important developmental period may form the pre-stage for cerebellar underdevelopment which is frequently observed in preterm infants and associated with various neurodevelopmental disorders in later life [2]. The alterations in the cerebellar strata following global HI were paralleled by marked loss and reduced dendritic arborisation of Purkinje cells (PC) which play a pivotal role in structural and functional organization of cerebellar circuitry [27, 28]. Developmental disturbances in the cerebellar cortex were more pronounced in the anterior lobe as compared to the posterior lobe after global HI. This spatial vulnerability might reflect distinct molecular properties acquired during development rendering the anterior lobe more vulnerable to injury [29, 30]. We observed hypomyelination and disorganization of myelin structure in the preterm cerebellum following global HI, which in this model similarly occurred in 
the cerebral subcortical white matter as previously reported [10]. Excessive activation of microglia is considered as one of the essential steps leading to white matter injury [31]. Accordingly, we found increased microgliosis in the white matter of the cerebellum following global HI. Our findings are in line with previous studies demonstrating white matter injury in the ovine cerebellum after repeated global HI [32] or an alternative pro-inflammatory stimulus such as systemic endotoxin [33] indicating the vulnerability of cerebellar white matter during early development.

We demonstrated that early systemic MAPC cell treatment modulated the pattern of injury in the preterm cerebellum after global HI, as indicated by protection of ultrastructure of cortical strata, prevention of PC loss and region-specific prevention of cerebellar white matter injury. These findings extend our previous results showing that the MAPC cells prevented hypomyelination of the periventricular white matter in the ovine preterm brain after global HI [10].

MAPC cells have been shown to possess strong anti-inflammatory and regenerative properties [16, 17]. Therefore, the first dose of MAPC cells was administered early after $\mathrm{HI}$, targeted to modulate the emerging inflammatory response, which plays an important role in the etiology of HIE [31]. The second dose of MAPC cells was administered in the sub-acute phase after global HI to support neuroregeneration after primary cell death has been initiated [31]. We showed that MAPC cells prevented hypomyelination and reduced PC cell injury to control levels after global HI. We consider it to be unlikely that regeneration of PC cells and oligodendrocytes (and remyelination) would occur in the short follow-up period of 7 days. Therefore, we consider that neuroprotection was primarily established by the first dose of MAPC cells that dampened the peripheral and cerebral inflammatory response, rather than regeneration of oligodendrocytes and PC cells by the second dose. Such pharmacological benefit of MAPC-driven immunomodulation was previously shown in experimental studies where MAPC cells modulated the cerebral and peripheral inflammatory response after various types of CNS injury, thereby providing an environment for recovery and repair of brain function and structure [16, 17]. More precisely, the neuroprotective effect was attributed to modulation of the peripheral inflammatory response in a spleen-mediated fashion $[17,34]$ and modulation of the cerebral inflammatory response by inducing a microglial-type switch from a pro- inflammatory M1 phenotype to an anti-inflammatory 
M2 phenotype [16, 17, 35]. Unfortunately, we cannot confirm the MAPC-induced switch towards a dominant neuroprotective M2 phenotype since ovine-specific reagents are currently not available to discriminate M1 from M2 microglia. Moreover, we were not able to incorporate various timepoints to assess the cerebral inflammatory and structural changes over time or to discriminate between the therapeutic effect of the first and second dose of MAPC cells. These limitations are inherent to limited experimental groups to make large animal experiments feasible.

We showed neuroprotection with an early treatment strategy, which was initiated 1 hour after global HI. Recent work in a similar ovine model of HIE indicated that later (12-24 hours after global HI) administration of stem cells similarly protected the preterm brain [36, 37]. Moreover, recent clinical data showed that MAPC cells are neuroprotective when administered 18-36 hours after ischemic stroke [13]. Based on these combined findings we expect that administration of MAPC cells to neonates at a clinically more feasible timepoint (24 hours) after the hypoxic-ischemic event will result in comparable neuroprotective effects as shown in our current study.

The work in this preclinical animal model enabled us to correlate microstructural histological changes with DTI. Although acute cerebellar injury was found in a large $(>80 \%)$ percentage of infants in human post-mortem studies [38], conventional MRI (e.g. T1- and T2 weighted imaging) is notoriously poor in detecting acute cerebellar injury [39]. We showed that DTI readily detected HI-induced injury and MAPC cellmediated microstructural improvement, both of which were concurrently demonstrated with histopathology. Furthermore, FA values correlated well with several relevant histopathological markers of cerebellar injury, further indicating the added value of assessing microstructural injury and repair with this advanced imaging technique. Our data suggest that DTI allows early detection of cerebellar alterations in pediatric patients. Therefore, we advocate the use of DTI to evaluate HI damage to the brain and to monitor the effects of future neuroprotective strategies in vivo in humans. However, not all histological changes in our study were detected by DTI and effect sizes appear to be more pronounced in histopathological analysis suggesting that even with advanced MRI techniques such as DTI, certain pathological phenomena could be underestimated. Currently, advanced imaging algorithms and post-processing methods are being 
developed, which will increase sensitivity and specificity of DTI and thereby further improve the clinical use of DTI as a biomarker of individual neurodevelopment and therapeutic effect in the future.

One important limitation of this large animal study is the low number of animals. The drop-out of one animal in the HI-MAPC group may have influenced therapeutic effect sizes. Given the relatively small animal numbers per group, we reported actual $p$ values and tended to interpret $p$ values between 0.05 and 0.1 as biologically relevant [40]. This assumption decreases the chance of a false-negative finding but increases the chance that one of these differences is a false-positive result [41]. Importantly, the observed treatment effects in the cerebellum were consistent with our previous experimental work showing MAPC-mediated neuroprotection after global HI [10].

MAPC cells have been approved for clinical use in adult CNS injury. A recent clinical trial in adult stroke patients showed that early administration of a single dose of MultiStem ${ }^{\circledR}$ (the clinical name for the MAPC derived product) was safe and significantly improved functional outcomes after 1-year follow-up [13]. An international multi-centered phase III trial further evaluating the neuroprotective potential of these cells in adult stroke patients has been initiated in 2018 (clinicaltrial.gov NCT03545607). Altogether, our findings of neuroprotection in the preterm cerebrum [10] and cerebellum may extend future clinical application of clinical-grade MAPC cells from adult stroke to neonatal brain injury.

In conclusion, this is the first study in a preclinical animal model that indicated that early systemic MAPC cell therapy induced microstructural improvement in the preterm cerebellum following global HI. Moreover, microstructural alterations in the cerebellum were detectable with DTI, indicating the added clinical value of this imaging technique. Our findings underline the potential of MAPC cell therapy for the treatment of preterm brain injury after $\mathrm{HI}$. 


\section{References}

1. Volpe JJ. Brain injury in premature infants: a complex amalgam of destructive and developmental disturbances. Lancet neurology. 2009;8:110-124.

2. Volpe JJ. Cerebellum of the premature infant: rapidly developing, vulnerable, clinically important. J Child Neurol. 2009;24:1085-1104.

3. Stoodley CJ, Limperopoulos C. Structure-function relationships in the developing cerebellum: Evidence from early-life cerebellar injury and neurodevelopmental disorders. Seminars in fetal \& neonatal medicine. 2016;21:356-364.

4. Hutton LC, Yan E, Yawno T, et al. Injury of the developing cerebellum: a brief review of the effects of endotoxin and asphyxial challenges in the late gestation sheep fetus. Cerebellum. 2014;13:777-786.

5. Kwan S, Boudes E, Gilbert G, et al. Injury to the Cerebellum in Term Asphyxiated Newborns Treated with Hypothermia. AJNR Am J Neuroradiol. 2015;36:1542-1549.

6. Johnsen SD, Bodensteiner JB, Lotze TE. Frequency and nature of cerebellar injury in the extremely premature survivor with cerebral palsy. J Child Neurol. 2005;20:60-64.

7. Pieterman K, White TJ, van den Bosch GE, et al. Cerebellar Growth Impairment Characterizes School-Aged Children Born Preterm without Perinatal Brain Lesions. AJNR. American journal of neuroradiology. 2018.

8. Bennet L, Tan S, Van den Heuij L, et al. Cell therapy for neonatal hypoxia-ischemia and cerebral palsy. Annals of neurology. 2012;71:589-600.

9. Jellema RK, Wolfs TG, Lima Passos V, et al. Mesenchymal stem cells induce T-cell tolerance and protect the preterm brain after global hypoxia-ischemia. PLoS ONE. 2013;8:e73031.

10. Jellema RK, Ophelders DR, Zwanenburg A, et al. Multipotent adult progenitor cells for hypoxic-ischemic injury in the preterm brain. J Neuroinflammation. 2015;12:241.

11. Boozer S, Lehman N, Lakshmipathy U, et al. Global Characterization and Genomic Stability of Human MultiStem, A Multipotent Adult Progenitor Cell. J Stem Cells. 2009;4:17-28.

12. Kovacsovics-Bankowski M, Mauch K, Raber A, et al. Pre-clinical safety testing supporting clinical use of allogeneic multipotent adult progenitor cells. Cytotherapy. 2008;10:730-742.

13. Hess DC, Wechsler LR, Clark WM, et al. Safety and efficacy of multipotent adult progenitor cells in acute ischaemic stroke (MASTERS): a randomised, double-blind, placebo-controlled, phase 2 trial. Lancet Neurol. 2017;16:360-368. 
14. Cunha JP, Leuckx G, Sterkendries $P$, et al. Human multipotent adult progenitor cells enhance islet function and revascularisation when co-transplanted as a composite pellet in a mouse model of diabetes. Diabetologia. 2017;60:134-142.

15. Mays RW, Borlongan CV, Yasuhara $T$, et al. Development of an allogeneic adherent stem cell therapy for treatment of ischemic stroke. J Exp Stroke Transl Med. 2010;3:34-46.

16. Busch SA, Hamilton JA, Horn KP, et al. Multipotent adult progenitor cells prevent macrophage-mediated axonal dieback and promote regrowth after spinal cord injury. The Journal of neuroscience : the official journal of the Society for Neuroscience. 2011;31:944-953.

17. DePaul MA, Palmer M, Lang BT, et al. Intravenous multipotent adult progenitor cell treatment decreases inflammation leading to functional recovery following spinal cord injury. Scientific reports. 2015;5:16795.

18. Back SA, Riddle A, Hohimer AR. Role of instrumented fetal sheep preparations in defining the pathogenesis of human periventricular white-matter injury. Journal of Child Neurology. 2006;21:582-589.

19. Leemans A, Jones DK. The B-matrix must be rotated when correcting for subject motion in DTI data. Magn Reson Med. 2009;61:1336-1349.

20. Irfanoglu MO, Walker L, Sarlls J, et al. Effects of image distortions originating from susceptibility variations and concomitant fields on diffusion MRI tractography results. Neuroimage. 2012;61:275-288.

21. Tax CM, Otte WM, Viergever MA, et al. REKINDLE: robust extraction of kurtosis INDices with linear estimation. Magn Reson Med. 2015;73:794-808.

22. Ophelders DR, Wolfs TG, Jellema RK, et al. Mesenchymal Stromal Cell-Derived Extracellular Vesicles Protect the Fetal Brain After Hypoxia-Ischemia. Stem Cells Transl Med. 2016;5:754-763.

23. Lordkipanidze T, Dunaevsky A. Purkinje cell dendrites grow in alignment with Bergmann glia. Glia. 2005;51:229-234.

24. Tolcos M, McDougall A, Shields A, et al. Intrauterine Growth Restriction Affects Cerebellar Granule Cells in the Developing Guinea Pig Brain. Developmental neuroscience. 2018;40:162-174.

25. Alexander AL, Lee JE, Lazar M, et al. Diffusion tensor imaging of the brain. Neurotherapeutics : the journal of the American Society for Experimental NeuroTherapeutics. 2007;4:316-329.

26. Gortner L, Felderhoff-Muser U, Monz D, et al. Regenerative therapies in neonatology: clinical perspectives. Klin Padiatr. 2012;224:233-240.

27. Daskalakis ZJ, Paradiso GO, Christensen BK, et al. Exploring the connectivity between the cerebellum and motor cortex in humans. The Journal of physiology. 2004;557:689-700.

28. Akkal D, Dum RP, Strick PL. Supplementary motor area and presupplementary motor area: targets of basal ganglia and cerebellar output. The Journal of neuroscience : the official journal of the Society for Neuroscience. 2007;27:10659-10673. 
29. Cerminara NL, Lang EJ, Sillitoe RV, et al. Redefining the cerebellar cortex as an assembly of non-uniform Purkinje cell microcircuits. Nature reviews. Neuroscience. 2015;16:79-93.

30. D'Mello AM, Stoodley CJ. Cerebro-cerebellar circuits in autism spectrum disorder. Frontiers in neuroscience. 2015;9:408.

31. Hagberg H, Mallard C, Ferriero DM, et al. The role of inflammation in perinatal brain injury. Nat Rev Neurol. 2015;11:192-208.

32. Castillo-Melendez M, Chow JA, Walker DW. Lipid peroxidation, caspase-3 immunoreactivity, and pyknosis in late-gestation fetal sheep brain after umbilical cord occlusion. Pediatric research. 2004;55:864-871.

33. Dean JM, Farrag D, Zahkouk SA, et al. Cerebellar white matter injury following systemic endotoxemia in preterm fetal sheep. Neuroscience. 2009;160:606-615.

34. Walker PA, Shah SK, Jimenez F, et al. Intravenous multipotent adult progenitor cell therapy for traumatic brain injury: preserving the blood brain barrier via an interaction with splenocytes. Experimental neurology. 2010;225:341-352.

35. Walker PA, Bedi S, Shah SK, et al. Intravenous multipotent adult progenitor cell therapy after traumatic brain injury: modulation of the resident microglia population. Journal of neuroinflammation. 2012;9:228.

36. Li J, Yawno T, Sutherland A, et al. Preterm white matter brain injury is prevented by early administration of umbilical cord blood cells. Exp Neurol. 2016;283:179-187.

37. van den Heuij LG, Fraser M, Miller SL, et al. Delayed intranasal infusion of human amnion epithelial cells improves white matter maturation after asphyxia in preterm fetal sheep. J Cereb Blood Flow Metab. 2017:271678X17729954.

38. Alderliesten ME, Peringa J, van der Hulst VP, et al. Perinatal mortality: clinical value of postmortem magnetic resonance imaging compared with autopsy in routine obstetric practice. BJOG : an international journal of obstetrics and gynaecology. 2003;110:378382.

39. Vermeulen RJ, Fetter WP, Hendrikx L, et al. Diffusion-weighted MRI in severe neonatal hypoxic ischaemia: the white cerebrum. Neuropediatrics. 2003;34:72-76.

40. Wasserstein RL, Schirm AL, Lazar NA. Moving to a World Beyond " $p<0.05$ ". Am Stat. 2019;73:1-19.

41. Amrhein V, Greenland S, McShane B. Scientists rise up against statistical significance. Nature. 2019;567:305-307. 
Ruth Gussenhoven*,Luise Klein*, Daan R.M.G. Ophelders, Denise H.J. Habets, Bernd Giebel, Boris W. Kramer, Leon J. Schurgers, Chris P.M. Reutelingsperger, and Tim G.A.M. Wolfs

Journal of Clinical Medicine 2019; 8[2]: 137.

*Authors contributed equally 


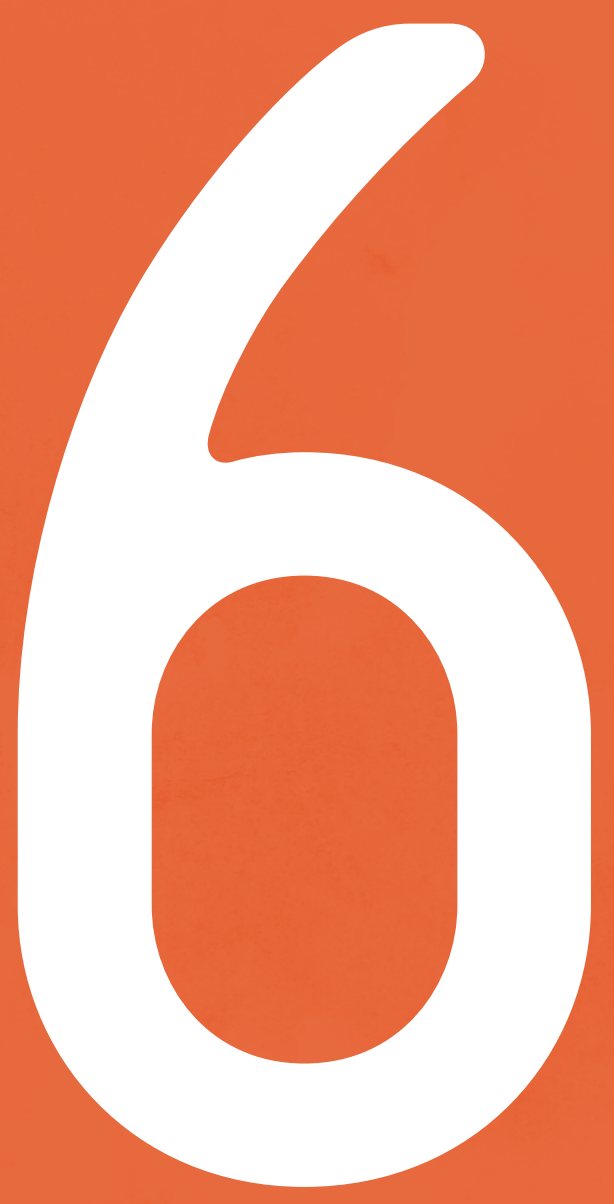

Annexin A1 as neuroprotective determinant for blood-brain barrier integrity in neonatal hypoxic-ischemic encephalopathy 


\section{Abstract}

Blood-brain barrier (BBB) disruption is associated with hypoxia-ischemia (HI) induced brain injury and life-long neurological pathologies. Treatment options are limited. Recently, we found that mesenchymal stem/stromal cell derived extracellular vesicles (MSC-EVs) protected the brain in ovine fetuses exposed to HI. We hypothesized that Annexin A1 (ANXA1), present in MSC-EVs, contributed to their therapeutic potential by targeting the ANXA1/Formyl peptide receptor (FPR), thereby preventing loss of the BBB integrity. Cerebral ANXA1 expression and leakage of albumin into the fetal ovine brain parenchyma after HI were analyzed by immunohistochemistry. For mechanistic insights, barrier integrity of primary fetal endothelial cells was assessed after oxygen-glucose deprivation (OGD) followed by treatment with MSC-EVs or human recombinant ANXA1 in the presence or absence of FPR inhibitors. Our study revealed that BBB integrity was compromised after HI which was improved by MSC-EVs containing ANXA1. Treatment with these MSC-EVs or ANXA1 improved BBB integrity after OGD, an effect abolished by FPR inhibitors. Furthermore, endogenous ANXA1 was depleted within 24 hours after induction of $\mathrm{HI}$ in cerebovasculature and ependyma and upregulated 72 hours after $\mathrm{HI}$ in microglia. Targeting ANXA1/FPR with ANXA1 in the immature brain has great potential in preventing BBB loss and concomitant brain injury following HI. 


\section{Introduction}

Hypoxic ischemic encephalopathy (HIE) in new-borns is defined as brain injury caused by insufficient blood flow and oxygen supply to the brain generally due to placental insufficiency or umbilical cord occlusion in the perinatal period [1]. HIE is therefore after preterm birth the most important recognized perinatal cause of neonatal mortality [2]. Accordingly, HIE has detrimental effects on brain development with severe long-term disabilities including cognitive impairments, mental retardation, epilepsy and cerebral palsy [1]. Treatment strategies are currently limited to hypothermia therapy which is only applicable in late preterm and term infants with HIE [3-6].

Understanding the underlying pathologic mechanisms of HIE is crucial for the development of new therapeutic interventions suited for preterm infants as well. Besides direct effects of hypoxia and reperfusion, and chronic neuroinflammation, the disruption of the blood-brain barrier (BBB) is increasingly recognized as an important cause of brain injury following neonatal HIE [5,7-10]. The BBB is mainly composed of endothelial cells (ECs) which form a barrier that communicates with both, the peripheral immune system and cells of the central nervous system, including pericytes, neurons, microglia and astrocytes, to maintain cerebral homeostasis [11,12]. There is evidence that the BBB is already functional early in development [13]. Following a hypoxic-ischemic (HI) insult excessive glutamate due to energy failure is released and free radicals are produced damaging cerebral cells including endothelial cells of the BBB [1,11]. Necrotic and apoptotic cells activate microglia that release cy tokines [1]. Microglia activation and oxidative stress contribute to changes in BBB permeability [14,15]. BBB permeability increases and perpetuates the neuroinflammatory response by enabling the infiltration of peripheral immune cells potentially aggravating the immune response and brain injury [16,17]. Clinical studies analyzing the BBB integrity demonstrate that global $\mathrm{HI}$ in neonates resulted in an increased CSF/serum albumin-ratio, an indicator for increased BBB permeability, and subsequent deregulation of cerebral homeostasis [7,8]. Moreover, more severe HIE clinical scores are associated with increased BBB permeability measurements, suggesting that brain injury is exacerbated due to BBB disruption [7]. In line, 
experimental rodent and ovine models for $\mathrm{HI}$ demonstrated that $\mathrm{HI}$ adversely affected the BBB. More precisely, brain pathology was closely related to areas of compromised BBB [18] and decrease in tight junction proteins was correlated with increased BBB permeability [9]. Altogether, the contribution of BBB injury in the pathophysiology of HIE indicates that strengthening the BBB integrity in the course of HI can attenuate brain injury. This idea mandates the understanding of the mechanisms regulating BBB integrity in order to exploit them as potential therapeutic targets.

We have previously shown in a fetal ovine HIE model that mesenchymalstem/ stromal cell derived extracellular vesicles (MSC-EVs) improved brain stem function and partially protected against hypomyelination. The therapeutic effects could not be attributed to anti-inflammatory effects of MSC-EVs [19]. Considering the role of the $\mathrm{BBB}$, we hypothesized that protective effects of MSC-EVs against global HI induced brain injury involve protection of the BBB.

Recently, Solito et al. identified Annexin A1 (ANXA1) to be an essential endogenous regulator of BBB integrity in neurodegenerative diseases [20]. This group furthermore showed that pharmacological administration of human recombinant ANXA1 (hrANXA1) ameliorated BBB permeability [20]. Classically, ANXA1 is appreciated as an important downstream effector molecule of anti-inflammatory glucocorticoid effects [21]. It activates antiinflammatory and pro-resolving mechanisms mainly as an agonist of formyl peptide receptors (FPRs), which are G protein-coupled receptors regulating host defense and inflammation [22,23]. In the adult brain, changes in ANXA1 expression are associated with neurodegenerative diseases related to BBB dysfunction such as Alzheimer's disease, Parkinson's disease and multiple sclerosis [20,24-27]. In the fetal brain, ANXA1 is expressed by microglia and ECs that form the BBB [12], suggesting a role of ANXA1 in cerebral inflammation and $\mathrm{BBB}$ regulation during development. Since annexins are frequently found in the proteome of extracellular vesicles [28-30], we hypothesized that MSC-EVs protected BBB integrity after global $\mathrm{HI}$ in new-borns, at least in part, through ANXA1 that is present in MSC-EVs [28-30]. We tested this in a preterm fetal ovine model in which brain injury was induced by global hypoxia-ischemia by transient umbilical cord occlusion (UCO) for 25 minutes $[16,17,19]$. Furthermore, we studied mechanistic insights in a widely accepted BBB in vitro model in which trans-endothelial electrical resistance (TEER) was measured on fetal primary ECs as a marker for endothelial integrity [31]. 


\section{Experimental Section}

\section{Study approval and experimental design}

All experimental procedures were performed in compliance with a protocol approved by the Animal Experiments Committee of Maastricht University Medical Center, NL (Mesenchymal stem cell-derived exosomes as a therapeutic intervention for hypoxia-ischemia in the preterm neonate, DEC 2012-064) and conducted in accordance with ARRIVE guidelines (https://www. nc3rs.org.uk/arrive-guidelines) and the Maastricht University guidelines on the Care and Use of the Laboratory Animals. The study design was described previously [19]. Briefly, individual fetuses $(n=37)$ of Texel pregnant ewes were randomly assigned to four different experimental groups: (1) sham umbilical cord occlusion (UCO) and saline treatment (sham-SAL), (2) sham UCO and MSC-EV treatment (sham-MSC-EVs), (3) UCO and saline treatment (HI-SAL) and (4) UCO and MSC-EV treatment (HI-MSC-EVs). All fetuses were instrumented at 102 days of gestational age (term 147 days of gestational age). An inflatable vascular occluder was inserted around the umbilical cord for induction of transient global hypoxia ischemia. An umbilical vessel catheter was placed in the femoral artery and brachial vein for measuring blood pressure and for the administration of MSC-EVs, respectively. After a recovery period of 4 days, fetuses were subjected to 25 minutes of sham occlusion or UCO through inflation of the vascular occluder. At the time of UCO, ovine brain development is comparable with 30 weeks of gestation in humans which coincides with the high-risk window of brain injury in preterm infants [32]. Fetuses assigned for MSC-EVs treatment received two boluses of MSC-EVs from $4 \times 107$ cell equivalents at 1 hour and 4 days following (sham) UCO. Control animals received an equal volume of sterile $0.9 \%$ sodium chloride (SAL) at similar time points. Fetuses were sacrificed 1 day $(n=10), 3$ days $(n=8)$ or 7 days $(\mathrm{n}=19)$ after (sham) UCO (Figure 1). The investigators performing the (sham) UCO, tissue sampling and post-mortem analysis were blinded to treatment allocation. The MSC-EV groups on day 7 are relatively small due to a dropout of $16 \%$ which was primarily restricted to the sham-MSC-EV group (i.e., 3 animals of the sham-MSC-EV group and 1 animal of the HI-MSC-EV) caused by a technical reason as reported previously [19]. 


\section{Mesenchymal stem/stromal cell-extracellular vesicles}

After informed consent according to the Declaration of Helsinki, bone marrow-derived MSCs were raised from human donated bone marrow, as described previously [33]. The MSC characteristics were verified by flow cytometry and conventional MSC differentiation assays [19,33]. MSC-conditioned media was harvested as described previously and EVs were harvested from MSC-conditioned medium through a polyethylene glycol (PEG) method in which following PEG precipitation the volume of the conditioned media is reduced by a low-speed centrifugation $(1500 \times \mathrm{g})$ after overnight incubation at $4{ }^{\circ} \mathrm{C}$. Obtained pellets were resuspended and washed in $0.9 \% \mathrm{NaCl}$ and following reprecipitation by ultracentrifugation solved in $1 \mathrm{~mL}$ aliquots, reflecting the MSC-EV yield of the supernatant of 4 x 107 MSCs [33]. MSC-EVs were characterized by Nanoparticle Tracking Analysis (NTA) and by western blots for CD81 and tumor susceptibility gene 101 (Tgs101), as described previously [33,34]. For our study, MSC-EVs were further characterized by tunable resistive pulse sensing (TRPS) and additional western blot analyses.

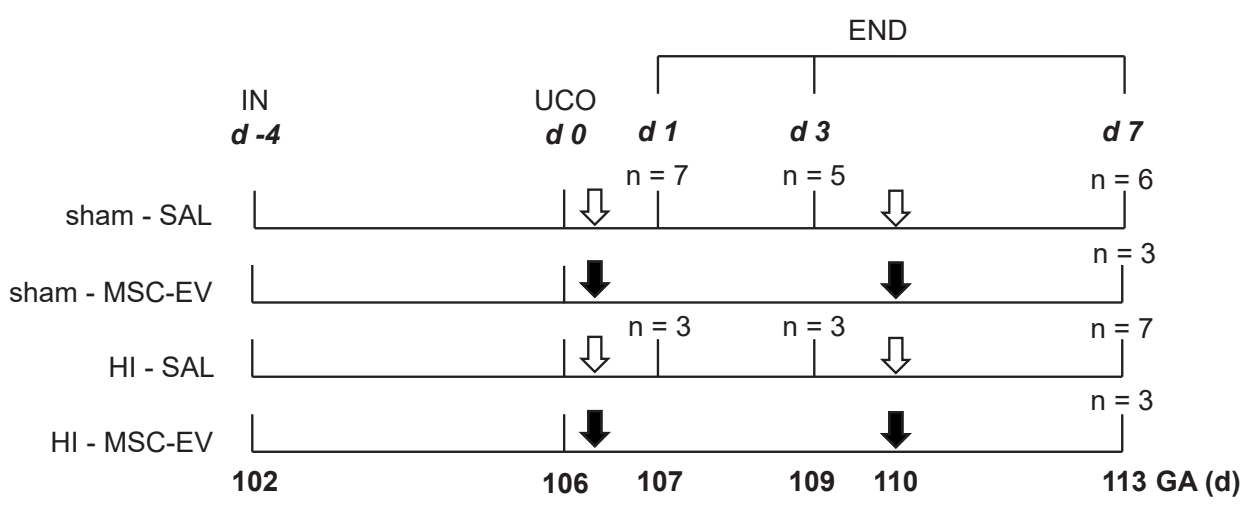

Figure 1. Experimental design. Fetuses were instrumented at GA 102 (IN; d-4). After 4 days of recovery, fetuses were subjected to 25 minutes of umbilical cord occlusion (UCO) or sham occlusion (0d). One hour and 4 days (110d GA) after (sham) UCO, fetuses received either intravenous MSC-EVs (2.0 x $10^{7} \mathrm{cell}$ equivalents; black arrows) or an equivalent volume of saline $0.9 \%$ (SAL; white arrows). After a $1 \mathrm{~d}, 3 \mathrm{~d}$ (sham-SAL and HI-SAL groups only) and 7d (all groups) reperfusion period, at 107d, 109d and 113d GA respectively, animals were sacrificed and brain tissue was collected. Abbreviations: $d$, day; END, end of experiment; GA, gestational age; HI, hypoxia-ischemia; IN, instrumentation; MSC-EV, mesenchymal stem cell-derived extracellular vesicle; UCO, umbilical cord occlusion, SAL, saline. 


\section{MSC-EV analysis using tunable resistive pulse sensing [TRPS]}

For in vitro experiments, amount of particles within the MSC-EVs were determined by TRPS using a qNano Gold with Izon Control Suite 3.2 Software and CPC100 calibration beads (Izon, Oxford, UK) by the Department of Medical Microbiology at Maastricht University. A NP150 nanopore [Izon, Oxford, UK) was coated using the Izon reagent kit for EV analysis according to the manufacturer's instructions. To obtain a stable baseline current, samples were diluted 1:100 in Solution Q (Izon, Oxford, UK) and to prevent frequent pore obstruction, 10\% (v/v) Solution G (Izon, Oxford, UK) was added. The NP150 nanopore was used at a stretch of $46.51 \mathrm{~mm}$ and a pressure of 6 mbar. Mean current for measuring was $128 \mathrm{nA}$ with a voltage of $0.42 \mathrm{~V}$. Recordings were stopped after 10 minutes with more than 400 blockades detected in both samples.

\section{Western blot of MSC-EVs}

MSC-EVs $\left(40 \mu \mathrm{L}, 1.76 \mathrm{mg} / \mathrm{mL}\right.$ protein concentration determined by Pierce ${ }^{\mathrm{TM}}$ BCA assay [Fisher Scientific, Landsmeer, NL]) were tested for Annexin A1 [ANXA1] by western blot analysis. Pure human platelet lysate (hPL), hPL centrifuged at $10.000 \times \mathrm{g}$ for 10 minutes and centrifuged lysate with subsequent filtration through a $0.2 \mu \mathrm{M}$ filter were used as negative control to ensure that no platelet derived ANXA1 was present in the MSC-EVs as MSCs were expanded in MSC basal media supplemented with 10\% hPL. Gel electrophoresis of samples was performed on a 10\% SDS-polyacrylaminde gel.

Separated samples including 200 ng human recombinant (hr]ANXA1 [35] and a broad protein marker [Biorad, Hercules, CA, USA] were transferred to a nitrocellulose membrane and probed with a 1:1000 dilution of anti-ANXA1 which was a kind gift from Prof. Mauro Perretti followed by an goat anti-rabbit-IgG-alkaline phosphatase [SigmaAldrich, St. Louis, MO, USA]. For visualisation the membrane was washed in a reaction buffer [0.1 M Tris- $\mathrm{HCl}, 0.1 \mathrm{M} \mathrm{NaCl}, 5 \mathrm{mM} \mathrm{MgCl}$ ] followed by incubation in substrate solution containing Nitrotetrazolium Blue chloride [Sigma-Aldrich, St. Louis, MO, USA] and 5-Bromo-4-chloro-3-indolyl phosphate disodium salt (Sigma-Aldrich, St. Louis, MO, USA]. The reaction was stopped by 0.5 M EDTA, and scans were taken by an Epson Perfection V300 Photo scanner [Supplementary Figure S1].

This western blot procedure was confirmed and extended. For this experiment, a dilution curve of 1, 3, 10 and 30 ng human recombinant [hr]ANXA1 [35] and a Dual color marker (Biorad, Hercules, CA, USA) were transferred to a nitrocellulose membrane and probed with a 1:2500 dilution of anti-ANXA1 (kind gift from Prof. Mauro Perretti) followed by an goat anti-mouse-IgG-HPR (Dako, Santa Clara, CA, USA). Samples were detected by Pierce $^{\text {TM }}$ ECL Western Blotting according to the manufacturer's protocol (Fisher Scientific, Landsmeer, NL]. Pictures were taken by an Odyssey scanner at different exposure times [50 sec and $1500 \mathrm{sec}$ ) (Li-cor, Bad-Homburg, Germany). 
(a)
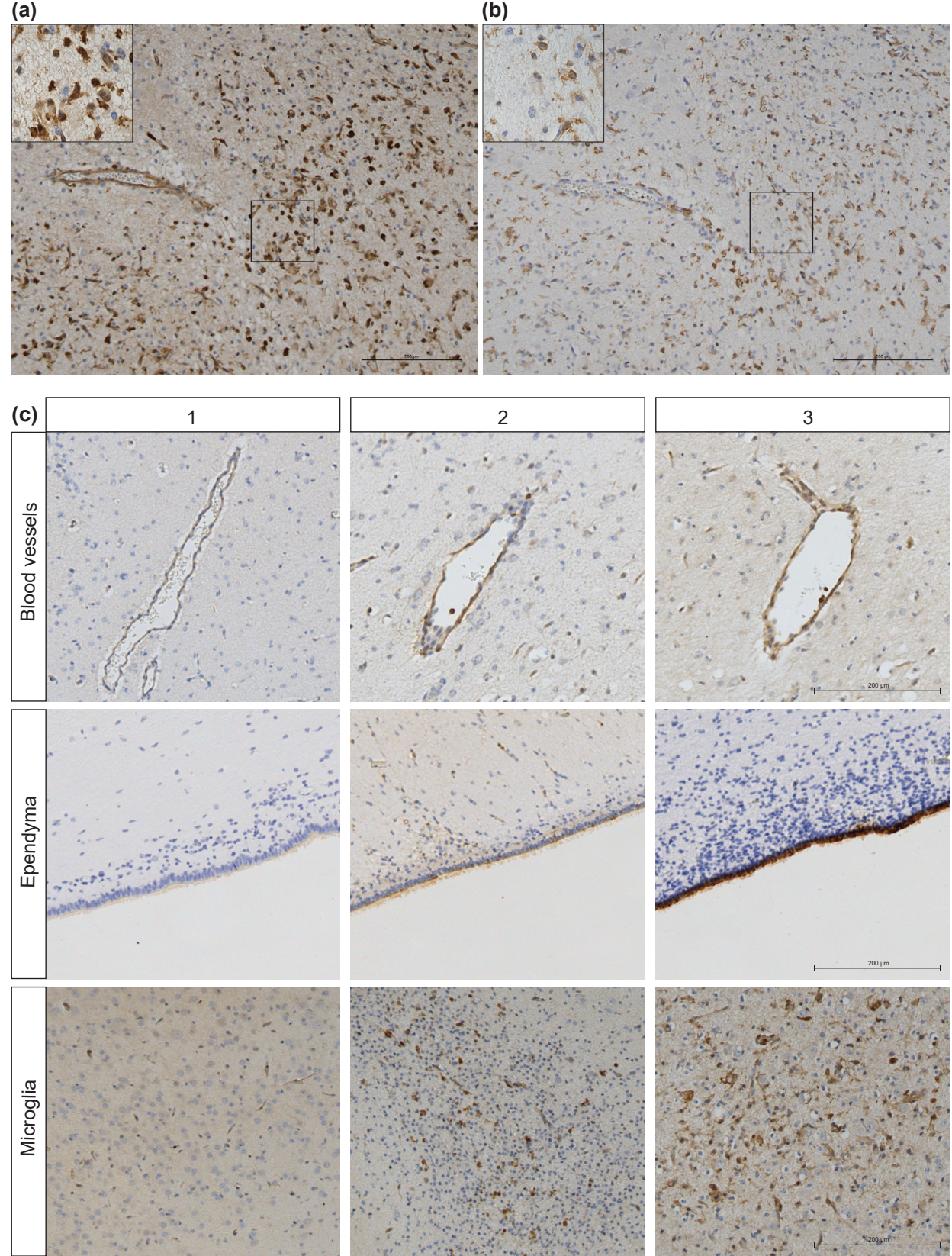
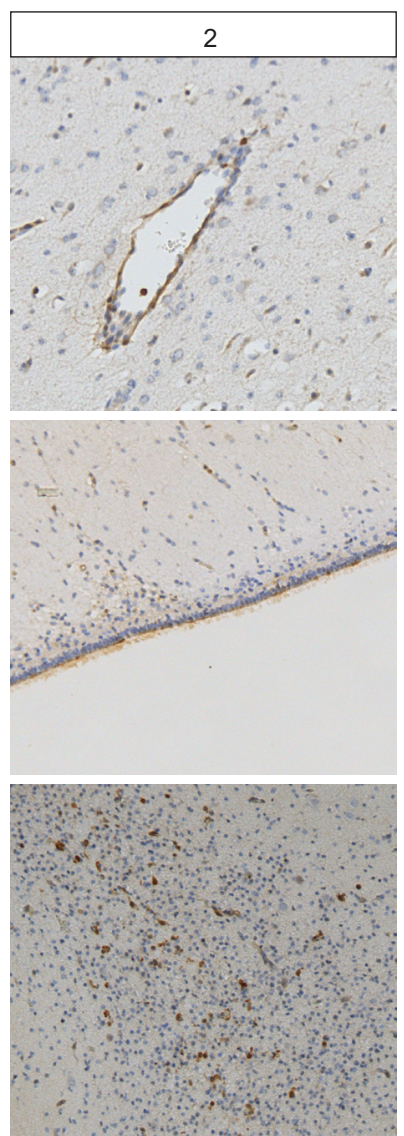
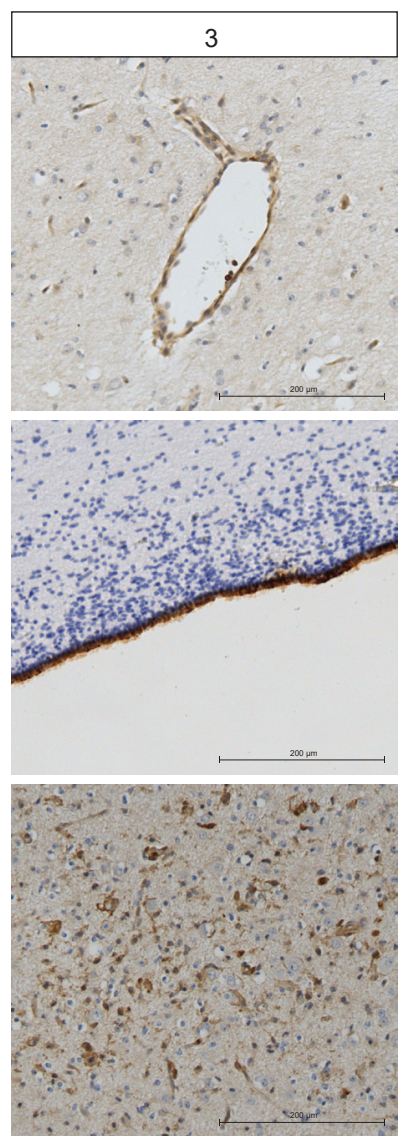

Figure 2. ANXA1 scoring system. a) ANXA1 is expressed within the white matter and b) co-localizes with IBA1 IR in adjacent sections (magnification 100x, scale bar $200 \mu \mathrm{m})$. c) ANXA1 IR scoring system (1, 2,3 ) of blood vessels, ependymal tissue and microglia (magnification 100x, scale bar $200 \mu \mathrm{m}$ ). 


\section{Immunohistochemistry and analysis}

Immediately after sacrifice, fetal brains were removed from skulls and weighed.

The right hemisphere was submersion fixated in ice-cold $4 \%$ paraformaldehyde for 3 months. After fixation, a predefined region containing the lateral ventricles, periventricular white matter and basal ganglia was embedded in paraffin and serial coronal sections ( 4 $\mathrm{m}$ ) were cut with a Leica RM2235 microtome (Leica Microsystems B.V., Amsterdam, NL). Coronal sections were stained for albumin as a marker for BBB leakage, ionized calcium binding adaptor molecule 1 (IBA1) as a general microglia marker and ANXA1. Endogenous peroxidase activity was quenched via incubation with $0.3 \%$ hydrogen peroxide dissolved in Tris-Buffered Saline (TBS). Antigen retrieval involved boiling tissues in a sodium citrate buffer $(\mathrm{pH}$ 6.0) using a microwave oven. Next, sections were incubated overnight with the primary polyclonal rabbit antiANXA1 (Abcam; Cambridge, UK, 1:100), anti-albumin (Accurate Chemical, Westbury, NY, USA; 1:2000), anti-IBA1 (Wako chemicals, Neuss, Germany; 1:1000) antibody at $4^{\circ} \mathrm{C}$, followed by incubation with a secondary polyclonal swine anti-rabbit biotin (Dako, Santa Clara, CA, USA; 1:200). The antibody specific staining was enhanced with a Vectastain ABC peroxidase elite kit (Vector Laboratories, Burlingame, CA, USA) followed by a 3,3'-diaminobenzidine (DAB) staining. Nuclei were counterstained with Mayer's haematoxylin.

Analysis of immunohistochemical stainings was done after taking digital images using a Leica DM2000 microscope with Leica Qwin Pro V3.4.0. Software (Leica Microsystems, Mannheim, Germany). Images of ANXA1 and IBA1 were taken at a magnification of 100x. Region of interest comprised the blood vessels, ependymal lining cells and white matter including microglial cells stained with IBA1. Assessment of ANXA1 IR in microglial cells was determined based on cellular phenotype and staining of adjacent sections with IBA1 co-localizing with ANXA1 IR (Figure 2 a and b). For the quantification of the intensity of ANXA1 IR we used a scoring system (1-3) to evaluate the immunoreactivity intensity of ANXA1 whereby score 1 comprised minor, 2 moderate and 3 intense immunoreactivity (IR) (Figure 2c). Scoring was complemented by analysis of area fractions, expressed as the percentage of positive staining relative to the total area using a standard threshold intensity, determined with Leica Qwin Pro V 3.5.1. Software (Leica, Rijswijk, NL). Same area fraction measurements were used for IBA1 IR assessment as described previously [16]. Moreover, the thickness of the ANXA1 positive stained periventricular area was measured with ImageJ Software version 1.48 . 
Analysis of albumin staining was done on 10 images (200x magnification) of similar sized blood vessels per animal. To evaluate the integrity of the BBB, albumin extravasation was scored with a (-) if no albumin was present in the cerebral parenchyma and a $(+)$ if positive albumin staining was present in the surrounding cerebral tissue of the blood vessel (see exemplary Figure 3b). These results are displayed as a percentage of albumin extravasation indicating leaky blood vessels.

\section{Endothelial cell isolation and culture}

Endothelial cells were a generous gift of Dr. Nynke van den Hoogen, University of Maastricht, Department of Mental Health and Neuroscience. Cells were isolated from brains of Sprague-Dawley rat pups sacrificed at day P3 by cervical dislocation accordingly to Bernas et al. 2010 [36]. The brain developmental stage of rodents on postnatal day 3 (P3) is comparable to preterm human infants [37]. Brains were dissected from the skull and meninges and large vessels were removed before trituration of the tissue by passing the fragments through decreasing pipet tips. Large fragments were filtered out by passing cell suspension through a $500 \mu \mathrm{M}$ strainer (pluriSelect, Leipzig, Germany). Cells in the flow-through were collected on a $30 \mu \mathrm{M}$ strainer (pluriSelect, Leipzig, Germany) and subsequently centrifuged at $51 \times \mathrm{g}$ for 10 minutes. The pellet was resuspended in DMEM-F12-glutamax (Fisher Scientific, Landsmeer, NL) supplemented with 10\% heat inactivated fetal bovine serum (FBS) (Sigma-Aldrich, St. Louis, MO, USA), 1\% antibioticantimycotic solution (Sigma-Aldrich, St. Louis, MO, USA), $50 \mu \mathrm{g} / \mathrm{mL}$ EC growth supplement (BD Biosciences, San Jose, CA, USA), $1 \mathrm{mg} / \mathrm{mL}$ heparin (Biochrom $\mathrm{GmbH}$, Berlin, Germany) and hydrocortisone $500 \mathrm{nM}$ (Stemcell Technologies, Cologne, Germany) and transferred into a T25 flask pre-coated with type-I-collagen (Corning Life Science, Oneonta, NY, USA). Culture expansion was allowed for approximately one month to achieve highly confluent cerebrovascular ECs showing minimal contamination by pericytes $(<5 \%)$. Characterization of the ECs to assess the purity of the cell population was performed by immunocytochemistry. Cells were grown on glass slides and stained for von Willebrand Factor (vWF) (Dako, Santa Clara, CA, USA), zona-occludens 1 (ZO-1) (Fisher Scientific, Landsmeer, NL) as EC markers and a-smooth muscle actin (a-SMA) as marker for pericytes (Sigma-Aldrich, St. Louis, MO, USA). Cells were fixated by incubation in $4 \%$ paraformaldehyde followed by blocking with either 3\% bovine serum albumin (BSA) or 10\% normal goat serum (NGS) in phosphate buffered saline (PBS). Cells were then incubated overnight with the primary antibody (ZO-1 1:50, a-SMA and vWF 
1:200) at $4^{\circ} \mathrm{C}$, followed by incubation with the appropriate alexa-fluor labelled secondary antibody (1:200). Nuclei were stained with DAPI and coverslips were mounted using fluorescent mounting medium (Dako, Santa Clara, CA, USA).

\section{Trans-endothelial electrical resistance [TEER]}

A cellular monolayer of ECs was cultured on semipermeable filter inserts (transwell, 12 wells) (Corning Life Science, Oneonta, NY, USA). TEER was measured as an established quantitative readout for barrier integrity [31] using an Epithelial Voltohmmeter (EVOM2, World Precision Instruments, Sarasota, FL, USA) with two chopstick electrodes, each containing a silversilver chloride pellet for measuring voltage and a silver pellet for passing current. Measurements of the resistance in ohm $(\Omega)$ across the cell layer were made on the semipermeable membrane by placing one electrode in the upper compartment and the other electrode in the lower compartment. Measurements were performed in duplo per insert and consistently conducted for several days, 30 minutes after culture media was changed and temperature was kept at $37^{\circ} \mathrm{C}$ before and between all measurements. Once values plateaued, the membrane reached confluency and further experiments could be performed (baseline measurement). When ECs reached confluency in the transwells, cells were randomly assigned to oxygen-glucose deprivation (OGD) or normoxia conditions and baseline TEER was measured. Normoxia controls were left in normal culture conditions. OGD was performed by changing the culture media with DMEM without glucose and glutamine (Life Technologies, Carlsbad, CA, USA) and exposing ECs to $0 \%$ oxygen in a hypoxic chamber at $37^{\circ} \mathrm{C}$ for 4 hours. After 4 hours of normoxia/OGD, medium was changed to culture media and TEER was measured followed by one or a combination of the treatments at the following concentrations: $1.1 \times 109$ MSC-EVs were given per well based on our TRPS analysis, hrANXA1 [31] ( $3 \mu \mathrm{M})$, FPR1/2 receptor blockers WRW4 $(10 \mu \mathrm{M}$, TOCRIS Bio-techne Ltd., Abingdon, UK) and cyclosporine $\mathrm{H}(1 \mu \mathrm{M}$, Sigma-Aldrich, St. Louis, MO, USA). Subsequently, TEER was measured in all groups at $0,3,6,12$ and 24 hours after normoxia/OGD. This setup resulted in following treatment groups: (1) no treatment, (2) MSC-EVs, (3) hrANXA1, (4) WRW4, (5) Cyclosporine H, (6) WRW4 + MSC-EV, (7) cyclosporine H + MSC-EVs, (8) WRW4 + hrANXA1, (9) cyclosporine H + hrANXA1. Cell culture experiments were repeated in duplo to test for reproducibility $(n=4)$. 


\section{Statistical analysis}

Regarding immunohistochemistry results, all values are shown as mean with $95 \%$ confidence interval (CI) or standard deviations (SD). Comparison between different experimental groups was performed with one- or two-way analysis of variance (ANOVA) and appropriate post-hoc testing. In case data were positively skewed, log-transformation was applied to obtain normal distributed data or non-parametric testing was performed.

Data from TEER measurements were obtained from 2 independent experiments each run in $n=2$ per treatment group making a total of $n=4$ per group. The absolute TEER values are reported as resistance across the EC layer on the semipermeable membrane in $\mathrm{Ohm}(\Omega)$. These values are averaged and presented as mean absolute TEER measurements $(\Omega)$ with $95 \% \mathrm{CI}$ and tested with a two-way analysis of variance (ANOVA) and appropriate post-hoc testing for significance.

Statistical analysis was performed with IBM SPSS Statistics Version 22.0 (IBM Corp., Armonk, NY, USA; SPSS) graphical design was performed using GraphPad Prism 5 (GraphPad Software, La Jolla, CA, USA; GraphPad Prism). Exact $p$-values are reported and statistical significance was accepted at $p<$ 0.05 .

\section{Results}

\section{MSC-EVs tended to prevent albumin leakage in the fetal ovine brain following global hypoxia-ischemia.}

Global HI induced a 34\%-38\% increase in albumin leakage compared to control animals (mean of $22 \%$ vs. $56 \%$ in sham-SAL vs. $7 d$ HI-SAL $p=0.054$ and mean of $22 \%$ vs. $60 \%$ of $3 \mathrm{~d}$ HI-SAL p $=0.088$ ) into the brain parenchyma (Figure 3a). At 7 days, in four out of seven (56\%) animals increased albumin leakage was found following HI compared to the mean of the sham-SAL group (sham-SAL vs. 7d HI-SAL, $\mathrm{p}=0.054$ ). No difference in albumin leakage was found at 1 day following HI (data not shown). MSC-EV treatment prevented albumin leakage on day 7 after reperfusion compared to the untreated control animals following HI (HI-SAL vs. 7d HI-MSC-EVs $\mathrm{p}=0.0501)$. MSC-EVs did not induced albumin leakage in sham conditions (sham-SAL vs sham-MSC-EVs $\mathrm{p}=0.613$ ) (Figure 3a). 
(a)

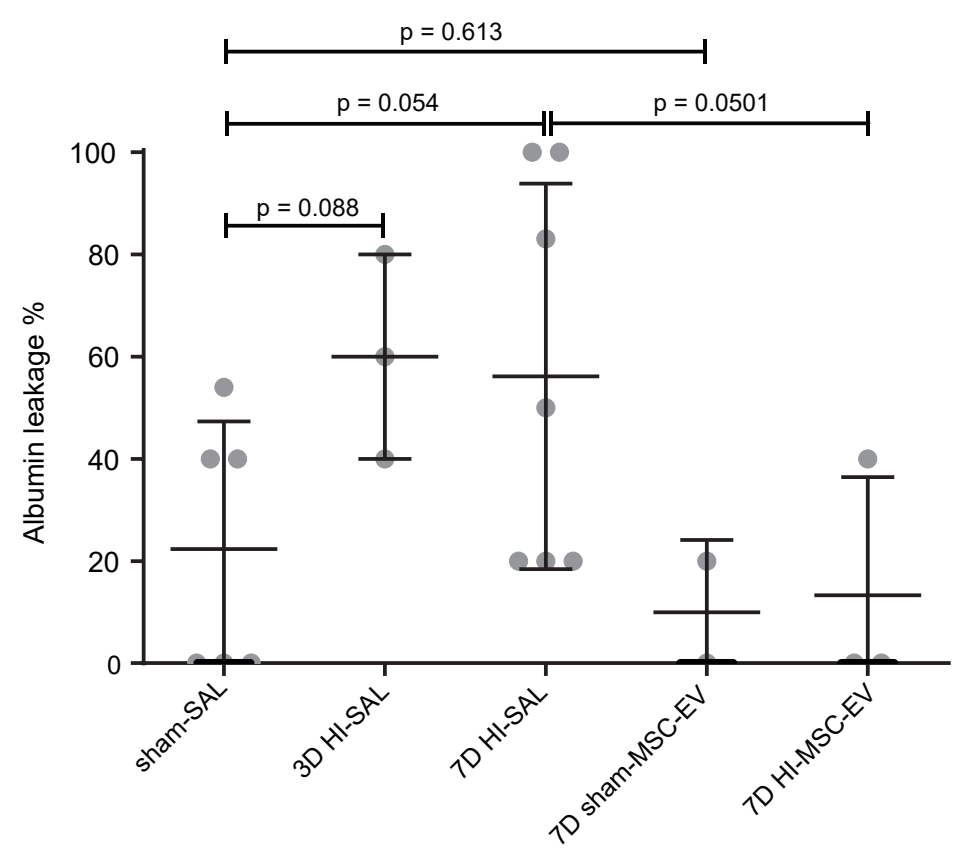

$\underline{6}$

(b)

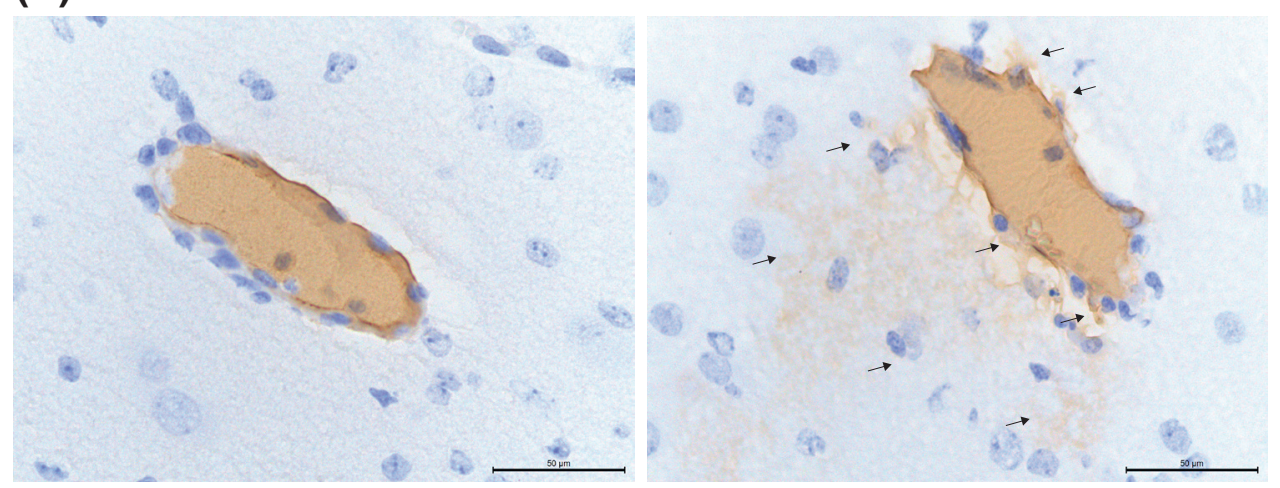

Figure 3. Albumin leakage into brain parenchyma. a) Percentage of albumin leakage inside the brain parenchyma is depicted. b) Immunohistochemical distribution of endogenous albumin. Representative vessels of a HI-MSC-EV animal showing albumin present inside the vessel (left) and albumin extravasation after $\mathrm{HI}$ (right) indicated by arrows. 400x magnification, scale bar $50 \mu \mathrm{m}$. 


\section{MSC-EVs and ANXA1 restored endothelial resistance / barrier integrity following oxygen-glucose deprivation in FPR dependent manner}

To study the potential mechanism underlying the protective effects of MSC-EVs observed in vivo, a model of primary fetal ECs isolated from rat brains at postnatal day 3 (P3) was used. Morphology of ECs was typically cobblestone-like (Figure 4a). Purity of EC culture was determined, 4 weeks after starting of the cell culture by immunocytochemical analysis of the vWF (Figure 4b), ZO-1 (Figure 4c) and pericyte marker a-SMA (Figure $4 \mathrm{~d})$. Baseline TEER values were approximately $150 \Omega$ per trans-well insert before experiments were initiated. After 4 hours of OGD, TEER values significantly decreased in each treatment group. Subsequently, MSC-EVs steadily increased TEER and values plateaued from 12 hours onwards at $122 \Omega$ (no treatment vs MSC-EVs 24 hours, $p=0.022$ ). These improved TEER values upon MSV-EV treatment were not detected when the FPR1 and FPR2 receptors were blocked by cyclosporine $\mathrm{H}$ and WRW4 (Figure 4e and f). As ANXA1 is an appreciated agonist of FPRs and known to strengthen BBB integrity in adult neuropathologies, we investigated whether ANXA1 is present in MSC-EVs and confirmed its presence by a western blot (Figure 4g). Taking pictures at low exposure times $(10-50 \mathrm{sec}), 10$ and $30 \mathrm{ng}$ hrANXA1 $(37 \mathrm{kDa})$ was already detectable. After increasing the exposure time (1500 sec), a fragment of $37 \mathrm{kDa}$ was detected in the MSC-EV sample whereas still no band was visible in the 1 and 3 ng hrANXA1 lanes or negative controls. Since the MSC-EV fraction used for the blot $(40 \mu \mathrm{L})$ contains more than $3 \mathrm{ng}$ and less than $10 \mathrm{ng}$ hrANXA1, $1 \mathrm{ml}$ of MSC-EVs isolated from 4 x 107 cells would contain (75-250 ng ANXA1/mL aliquot MSC-EVs. In line, a physiological relevant amount of ANXA1 in MSCs was found within the same ng range [30].

Subsequently, we tested whether BBB integrity in our in vitro model was improved after OGD in response to hrANXA1. Similar to MSC-EV treatment, hrANXA1 significantly improved TEER values after OGD (no treatment vs MSC-EVs 24 hours, $\mathrm{p}=0.017$ ), which was prevented in the presence of FPR inhibitors (Figure $4 \mathrm{~h}$ and i). 


\section{ANXA1 is widely expressed in the preterm ovine brain and the expression decreases acutely after global hypoxia-ischemia}

We investigated the temporary dynamics of ANXA1 expression within cerebral blood vessels, ependymal lining and microglia in vivo after HI. We found that 1 day after global HI, ANXA1 IR decreased significantly in blood vessels (1d sham-SAL vs. 1d HI-SAL $p=0.032$ ) and ependymal lining cells (1d sham-SAL vs. $1 \mathrm{~d}$ HI-SAL $\mathrm{p}=0.007)$ compared to controls, whereas at day 3 and day 7 seven ANXA1 expression were normalized (3d sham-SAL vs. 3d HI-SAL $\mathrm{p}=0.880$ and $\mathrm{p}=0.975$; 7d sham-SAL vs. 7d HI-SAL $\mathrm{p}=0.100$ and $\mathrm{p}=0.894$ ) (Figure $5 \mathrm{a}$ and $\mathrm{b}$ ). Systemic administration of MSC-EVs did not change ANXA1 expression 7 days after (sham) UCO in cerebral vasculature, ependyma and microglia (7d HI-SAL vs. 7d HI-MSC-EVs $\mathrm{p}=0.794$, 7d HI-SAL vs. 7d HI-MSC-EVs $\mathrm{p}=0.603$, 7d HI-SAL vs. 7d HI-MSC-EVs p = 0.999) (Supplementary Figure S2).

Since ANXA1 is expressed by microglia, we assessed IBA1 IR and ANXA1 IR in adjacent sections in the same regions of interest as illustrated in Figure 5c. In these white matter regions microglia were abundantly present and we found that at 3 days after global HI ANXA1 expression was significantly increased compared to time-matched controls (3d sham-SAL vs. 3d HI-SAL $\mathrm{p}=0.047$ ) (Figure 5c). Moreover, analysis of area fraction of IBA-1 IR showed that increased microglial activity was found at 3 and 7 days following HI (data not shown) which is in line with previous studies [16,19,38,39]. 
(a)

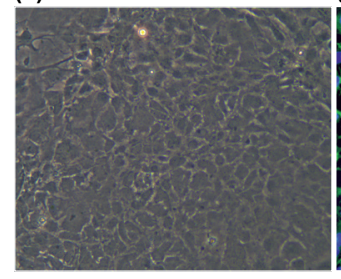

(b)
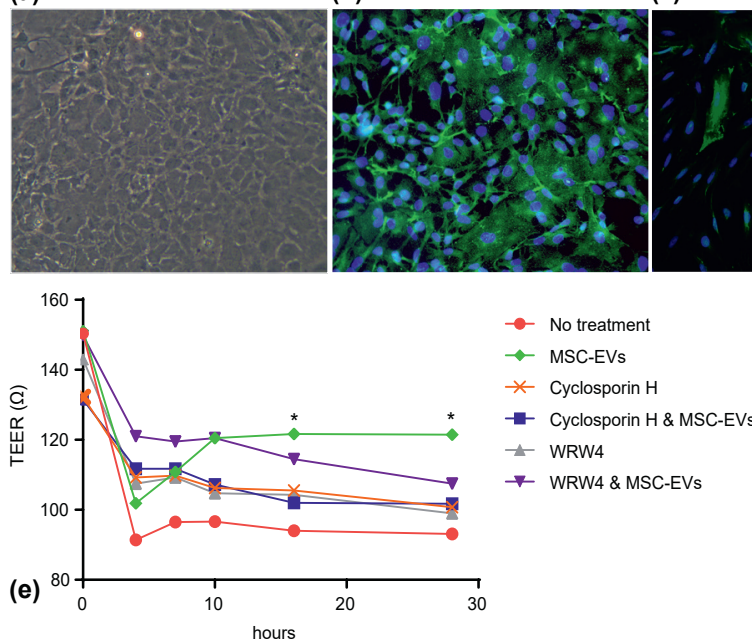

1.

$\begin{array}{llll}1 & 3 & 10 & 30\end{array}$

(g)

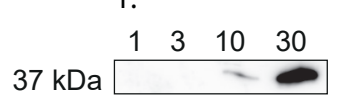

2.

MSC-EVs

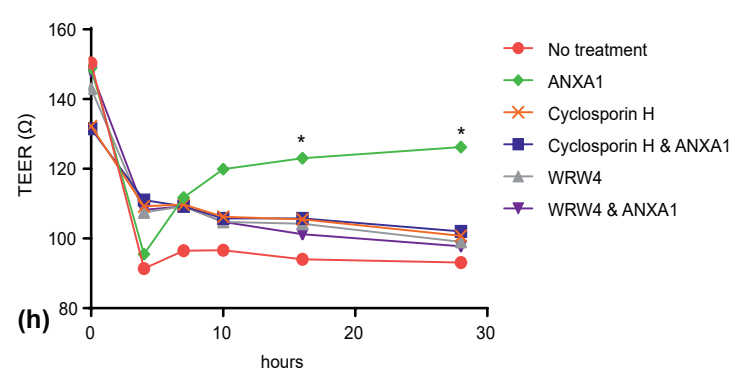

(c)

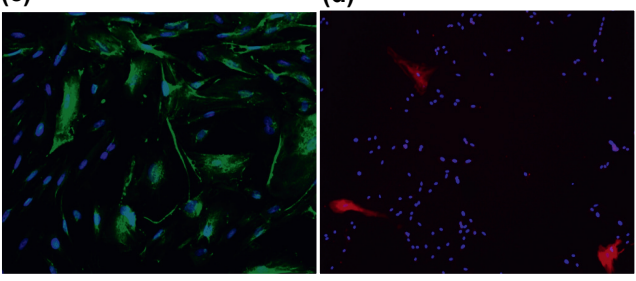

(d)
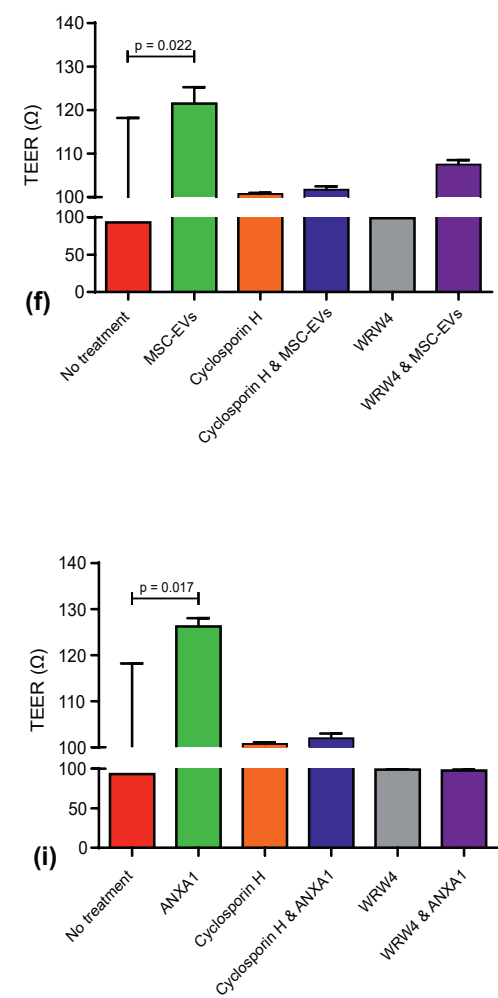

Figure 4. MSC-EVs and ANXA1 prevent loss of BBB integrity in vitro after OGD via FPRs. Characterization of primary fetal rat endothelial cell culture by a) bright-field microscopy (100x magnification) and b) immunocy tochemistry for vWF (200x magnification) c) immunocytochemistry for ZO-1 (200x magnification) d) immunocytochemistry for $\alpha$-SMA (40x magnification). e) Baseline TEER measurements were taken before initiation of OGD (0 hours). Four hours after OGD, fetal rat endothelial cells were treated with MSC-EVs and/ or FPR inhibitors and followed up for 3, 6, 12 and 24 hours (n=4). f) TEER endpoint measurement 24 hours after OGD and MSC-EV treatment (28 hours absolute time) in the presence or absence of FPR inhibitors $(\mathrm{n}=4) ;{ }^{*}=\mathrm{p}<0.05$. g) Western blot analyses to detect ANXA1 in MSC-EVs. g1. After 50 sec of exposure time, a weak and intense fragment was detected in the 10 and $30 \mathrm{ng}$ hrANXA1 lanes respectively whereas no signal was detected when MSC-EVs (not shown) or 1 and 3 ng hrANXA1 were loaded per lane. g2. After $1500 \mathrm{sec}$ of exposure time, endogenous ANXA1 $(37 \mathrm{kDa})$ was detected in lysate of MSC-EVs but not in MSC culture medium controls (pure hPL, pure hPL lysate $+10.000 \mathrm{xg}$ for 10 minutes, pure $\mathrm{hP}+10.000 \mathrm{xg}$ for 10 minutes $+0.2 \mu \mathrm{M}$ filtered) which were used as negative controls (not shown). h) Baseline TEER measurements were taken before initiation of OGD (0 hours). Four hours after OGD, fetal rat endothelial cells were treated with hrANXA1 and/or FPR inhibitors and followed up for 3, 6, 12 and 24 hours (n=4). i) TEER endpoint measurement, 24 hours after OGD and hrANXA1 treatment (28 hours absolute time) in the presence or absence of FPR inhibitors $(n=4) ;{ }^{*}=p<0.05$. magnification, scale bar $50 \mu \mathrm{m}$. 

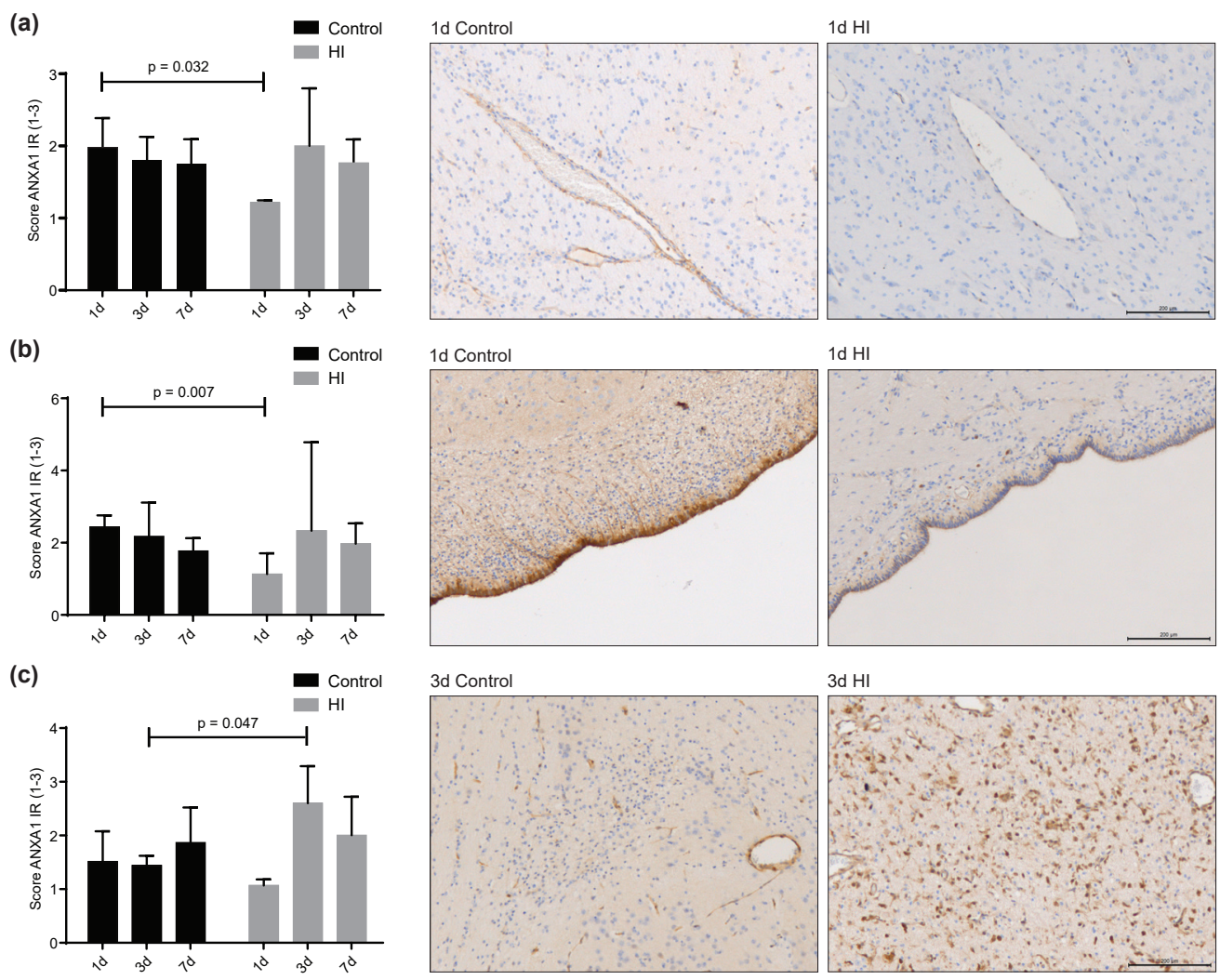

Figure 5. Temporal expression of ANXA1 on 1, 3 and 7 days after $\mathrm{HI}$ in cerebrovasculature, ependymal lining and microglia. a) ANXA1 expression in cerebrovasculature and representative picture of ANXA1 loss in vasculature 1 day after HI compared to control. b) Endogenous ANXA1 over time in ependymal lining and representative picture of ANXA1 loss in ependymal lining 1 day after HI compared to control. c) Endogenous ANXA1 expression in microglia over time and representative pictures of ANXA1 increase in microglia 3 days after HI compared to control. 100x magnification, scale bar $200 \mu \mathrm{m}$. 


\section{Discussion}

We show in our translational fetal sheep model of global HI that the BBB integrity is compromised, as indicated by an increased albumin leakage into the brain parenchyma. This is in line with clinical reports and studies with fetal animals in which loss of BBB integrity occurred after HI [7,9,10,18]. BBB dysfunction is one of the key mediators in adult neurological disorders including multiple sclerosis (MS), stroke, Alzheimer's and Parkinson's disease since it facilitates immune cell infiltration into the central nervous system (CNS), which act as drivers of cerebral inflammation and subsequent brain injury [24-27,40]. In the fetal situation, HI results in a multifactorial cascade of detrimental events, of which BBB dysfunction similar to adult neuropathologies is identified as one of the contributors to cerebral injury leading to life-long cognitive and sensory motoric disabilities [7,8]. In addition to direct BBB dysfunction, multiple phases of energy failure following global HI lead to cell death and release of excitotoxic molecules, eliciting a strong pro-inflammatory response by microglia that secrete cytokines and reactive oxygen species that exacerbate existing BBB damage [1,14,15,25,41-43]. Moreover, systemic inflammation, induced by a global HI insult, can increase the BBB permeability, allowing inflammatory mediators such as cytokines and inflammatory cells to enter the parenchyma, thereby contributing to disease progression. Evidence for such second hit in our global HI model was previously shown by recruitment of immune cells into the circulation within 24 hours following global $\mathrm{HI}$ with a subsequent marked cerebral influx of neutrophils and T-cells [16]. The influx of peripheral immune cells after cerebral ischemia is considered to further aggravate acute inflammation of the brain that was initiated by immediate cell death and microglial activation [42-44]. This emphasizes that disturbance of BBB integrity by $\mathrm{HI}$ and peripheral and cerebral inflammation act synergistically, leading to a self-enhancing loop of inflammation/brain damage which needs to be contained. Hence, targeting molecular pathways regulating BBB integrity appears a logical strategy to combat brain injury following HI.

We have previously shown in our fetal sheep model of global HI that 
pharmacological administration of MSC-EVs resulted in partial protection against hypomyelination. These therapeutic effects could not be explained by anti-inflammatory effects of the MSC-EVs [19]. Therefore, we focused on an alternative explanation for pharmacological effects of MSC-EVs, being restoring the injured BBB by targeting the ANXA1/ FPR-axis following HI. In the current study, we report that MSC-EVs protect the BBB that was compromised by global HI in our fetal sheep model. We conclude from our previous [19] and current findings that the protective effects of MSC-EVs arise, at least in part, from their protective actions on the BBB. Interestingly, recent studies of adult traumatic brain injury and stroke models showed that stem cell therapy diminished trauma and HI-induced BBB leakage [44,45]. Although our combined findings indicate that ANXA1driven inhibition of cerebral inflammation is not a plausible explanation for the pharmacological effects of MSC-EVs, we cannot rule out the possibility that microglia are part of the underlying working mechanism. More precisely, Solito et al found that ANXA1 plays an important role in controlling non-inflammatory phagocytosis of apoptotic cells and promoting resolution of inflammation in models of Alzheimer's disease [25]. An annexin-driven switch into M2 microglia that typically express such characteristics is a realistic scenario that warrants further investigation in a follow up study where neuroprotective effects of ANXA1 will be directly tested in vivo.

The most important finding of this study was that MSC-EV mediated protection of BBB integrity (after OGD) was dependent on FPR signaling and that MSC-EVs contain the potent FPR agonist ANXA1. ANXA1 is a multifunctional molecule originally identified as anti-inflammatory/ pro-resolving mediator and more recently also as a crucial regulator of BBB integrity [20,21]. In particular, ANXA1 KO mice exhibit increased BBB permeability compared to WT mice due to disrupted inter-EC tight junctions [20]. ANXA1 regulates the BBB by binding to the FPR2 receptor that in turn inhibits the activity of RhoA, subsequently promoting cytoskeletal stability and enhancing tight junction formation [20,46,47]. Blocking of FPR signaling by cyclosporine $\mathrm{H}$ or WRW4 leads to activation of RhoA that might induce a leaky BBB by destabilizing $\beta$-actin cytoskeleton. RhoA activity, inhibited by ANXA1, was also identified to induce BBB permeability in AD supporting the role of ANXA1 to regulate BBB integrity by this pathway [48]. Another study proposed FPR/ANXA1 interactions in improving BBB integrity, in a model studying sexual dimorphism in systemic inflammation [49]. Exclusively in young female mice ANXA1 signaling, downstream of estrogen, prevented inflammation-induced BBB tight junction breakdown and limited lymphocyte 
extravasation into the brain parenchyma [49]. Similar sexual dimorphism in susceptibility of $\mathrm{HI}$ induced preterm brain injury is also observed in clinic and experimental models which found females to be better protected from premature brain damage and sequelae compared to males [50-52].

In accordance with previous reports, we found ANXA1 to be expressed by fetal microglia, BBB endothelial and ependymal cells [12,53,54]. The mechanism by which endogenous ANXA1 regulates BBB integrity in brain microvascular ECs is considered to occur by binding to $\beta$-actin, thereby promoting cytoskeleton formation, which serves as an anchor linking $\beta$-actin to the plasma membrane, facilitating establishment of tight junctions between ECs [20].

Since ependymal cells, which form the basis of the brain-CSF interface, fulfil a similar barrier function during development as ECs of the BBB $[13,55,56]$ it is likely that ANXA1 exerts the same regulatory function in these cells. Neuroependymal cells of the fetus are connected by strap junctions that restrict the influx of larger molecules from the CSF into the brain [13,55-57] and disruption might cause uncontrolled passage of larger molecules between the brain and the CSF. This tempts us to speculate that loss of ANXA1 within the first 24 hours after $\mathrm{HI}$ might lead to a weakened brain-CSF barrier function. For future research, determination of albumin in CSF immediately after HI insult might give insights into the function of ANXA1 in ependymal lining. We aimed to measure albumin in CSF but unfortunately, this analysis was hampered by blood contamination during sampling of CSF in a number of samples.

An important observation in this study is the acute, transient decrease of endogenous ANXA1 expression of the cerebral vasculature following global $\mathrm{HI}$ indicating that an important endogenous protection of the BBB is abrogated which might destabilize BBB integrity promoting the vicious cycle of inflammation/brain damage. The clinical consequences of ANXA1 loss are supported by earlier findings, showing that depletion of ANXA1 was also detected in cerebrovascular capillaries and serum of MS and AD patients and respective experimental models in which a disrupted BBB is a major requirement for the onset of disease [20,48].

Besides acute ANXA1 loss in cerebrovasculature and ependymal cells, we found an increase in ANXA1 expression of microglia three days after HI compared to time-matched control animals. The increase of endogenous 
ANXA1 in microglia after the acute phase might be part of controlled resolution of inflammation. ANXA1 facilitates phagocytosis of apoptotic cells and debris by microglia without eliciting a pro-inflammatory response $[25,43,47,58]$. Furthermore, ANXA1 skews macrophages from a pro-inflammatory towards an anti-inflammatory phenotype [59-61]. The specific and acute loss of endogenous ANXA1 following HI in cells lining the BBB suggests the idea that the BBB is the primary site affected by HI, highlighting it as the most obvious therapeutic target in $\mathrm{HI}$.

A limitation of our in vitro study is that we cannot completely rule out that primary cells might lose characteristics during culturing. However, there is evidence that cerebral endothelial cells maintain specific morphology in culture which is especially the case with the endothelium from embryonal origin [62]. In particular, fetal endothelial cells did not show signs of cellular aging; cells retained ultrastructural characteristics and cells did not change lectin binding pattern [62]. Furthermore, there are several differences between adult and fetal brain endothelial cells i.e. adult and fetal endothelial cells differ in growth rate and expression of endothelial markers [63]. Also functional differences have been reported which are important to be mentioned such as different reaction to tumor-conditioned media [63] and neonatal endothelial cells display a lower barrier integrity compared to adult cells measured by TEER [64].

Given the relative small animal numbers per group, which is an inherent limitation to large animal models, we report actual $\mathrm{P}$ values and tend to interpret $\mathrm{P}$ values between 0.05 and 0.1 as biologically relevant. This assumption will decrease the chance of a false negative finding but increases the chance that one of these differences is a false positive result.

Together our data in this proof of concept study support the notion that the ANXA1/FPR axis is a therapeutic target to treat fetuses exposed to HI of the brain and that hrANXA1 is a potential therapeutic agent especially with regard to BBB function. Our current understanding of the pathologic course of $\mathrm{HI}$ is described by a primary phase during/after $\mathrm{HI}$ that is characterized by reduction in cerebral blood flow and subsequent oxygen/nutrient shortage resulting in severe tissue damage. The primary phase is followed by a latent/recovery phase ( $\sim 6$ hours after $\mathrm{HI})$ in which cerebral blood flow is restored leading to a secondary energy failure phase 6-72 hours after the initial insult $[1,4,6]$. The latent phase is believed to be the optimal window of 
therapeutic interventions $[1,4,6]$. Considering that ANXA1 depletion occurs within the first 72 hours upon ischemia in cerebrovasculature and ependyma, supplementation of exogenous ANXA1 should be conducted within the pharmacological window of opportunity of at most three days after the HI insult but preferably earlier.

\section{Supplementary Materials}

The following supplementary material is available online at www.mdpi.com/ xxx/s1, Figure S1 Western blot of MSC-EVs and negative controls for ANXA1, Figure S2 ANXA1 IR within cerebral blood vessels, ependyma and microglia in response to MSC-EV treatment 7 days after $\mathrm{HI}$ or sham-HI.

\section{Funding}

This work was supported by the Kinderonderzoekfonds Limburg (T.G.A.M.W.). 


\section{References}

1. Allen, K.A.; Brandon, D.H. Hypoxic Ischemic Encephalopathy: Pathophysiology and Experimental Treatments. Newborn Infant Nurs Rev 2011, 11, 125-133, doi:10.1053/j. nainr.2011.07.004.

2. Pathirana, J.; Muñoz, F.M.; Abbing-Karahagopian, V.; Bhat, N.; Harris, T.; Kapoor, A.; Keene, D.L.; Mangili, A.; Padula, M.A.; Pande, S.L., et al. Neonatal death: Case definition \& guidelines for data collection, analysis, and presentation of immunization safety data. Vaccine 2016, 34, 6027-6037, doi:10.1016/j. vaccine.2016.03.040.

3. Gopagondanahalli, K.R.; Li, J.; Fahey, M.C.; Hunt, R.W.; Jenkin, G.; Miller, S.L.; Malhotra, A. Preterm Hypoxic-Ischemic Encephalopathy. Frontiers in pediatrics 2016, 4, 114, doi:10.3389/fped.2016.00114.

4. Davidson, J.O.; Wassink, G.; van den Heuij, L.G.; Bennet, L.; Gunn, A.J. Therapeutic Hypothermia for Neonatal Hypoxic-Ischemic Encephalopathy - Where to from Here? Front Neurol 2015, 6, 198, doi:10.3389/fneur.2015.00198.

5. Rao, R.; Trivedi, S.; Vesoulis, Z.; Liao, S.M.; Smyser, C.D.; Mathur, A.M. Safety and Short-Term Outcomes of Therapeutic Hypothermia in Preterm Neonates 34-35 Weeks Gestational Age with Hypoxic-Ischemic Encephalopathy. J Pediatr 2017, 183, 37-42, doi:10.1016/j.jpeds.2016.11.019.

6. Cotten, C.M.; Shankaran, S. Hypothermia for hypoxic-ischemic encephalopathy. Expert Rev Obstet Gynecol 2010, 5, 227-239, doi:10.1586/eog.10.7.

7. Kumar, A.; Mittal, R.; Khanna, H.D.; Basu, S. Free radical injury and blood-brain barrier permeability in hypoxic-ischemic encephalopathy. Pediatrics 2008, 122, e722-727, doi:10.1542/peds.2008-0269.

8. Lee, W.L.A.; Michael-Titus, A.T; Shah, D.K. Hypoxic-Ischaemic Encephalopathy and the Blood-Brain Barrier in Neonates. Dev Neurosci 2017, 39, 49-58, doi:10.1159/000467392.

9. Chen, X.; Threlkeld, S.W.; Cummings, E.E.; Juan, I.; Makeyev, O.; Besio, W.G.; Gaitanis, J.; Banks, W.A.; Sadowska, G.B.; Stonestreet, B.S. Ischemia-reperfusion impairs blood-brain barrier function and alters tight junction protein expression in the ovine fetus. Neuroscience 2012, 226, 89-100, doi:10.1016/j.neuroscience.2012.08.043.

10. Baburamani, A.A.; Castillo-Melendez, M.; Walker, D.W. VEGF expression and microvascular responses to severe transient hypoxia in the fetal sheep brain. Pediatr Res 2013, 73, 310-316, doi:10.1038/pr.2012.191. 
11. Moretti, R.; Pansiot, J.; Bettati, D.; Strazielle, N.; Ghersi-Egea, J.F.; Damante, G.; Fleiss, B.; Titomanlio, L.; Gressens, P. Blood-brain barrier dysfunction in disorders of the developing brain. Front Neurosci 2015, 9, 40, doi:10.3389/fnins.2015.00040.

12. McArthur, S.; Loiola, R.A.; Maggioli, E.; Errede, M.; Virgintino, D.; Solito, E. The restorative role of annexin A1 at the blood-brain barrier. Fluids Barriers CNS 2016, 13, 17, doi:10.1186/ s12987-016-0043-0.

13. Saunders, N.R.; Daneman, R.; Dziegielewska, K.M.; Liddelow, S.A. Transporters of the blood-brain and blood-CSF interfaces in development and in the adult. Molecular aspects of medicine 2013, 34, 742-752, doi:10.1016/j.mam.2012.11.006.

14. Smyth, L.C.D.; Rustenhoven, J.; Park, T.I.; Schweder, P.; Jansson, D.; Heppner, P.A.; O'Carroll, S.J.; Mee, E.W.; Faull, R.L.M.; Curtis, M., et al. Unique and shared inflammatory profiles of human brain endothelia and pericytes. J Neuroinflammation 2018, 15, 138, doi:10.1186/ s12974-018-1167-8.

15. da Fonseca, A.C.; Matias, D.; Garcia, C.; Amaral, R.; Geraldo, L.H.; Freitas, C.; Lima, F.R. The impact of microglial activation on blood-brain barrier in brain diseases. Front Cell Neurosci 2014, 8, 362, doi:10.3389/fncel.2014.00362.

16. Jellema, R.K.; Lima Passos, V.; Zwanenburg, A.; Ophelders, D.R.M.G.; De Munter, S.; Vanderlocht, J.; Germeraad, W.T.V.; Kuypers, E.; Collins, J.J.P.; Cleutjens, J.P.M., et al. Cerebral inflammation and mobilization of the peripheral immune system following global hypoxia-ischemia in preterm sheep. Journal of Neuroinflammation 2013, 10, 807, doi:10.1186/1742-2094-10-13.

17. Jellema, R.K.; Wolfs, T.G.A.M.; Lima Passos, V.; Zwanenburg, A.; Ophelders, D.R.M.G.; Kuypers, E.; Hopman, A.H.N.; Dudink, J.; Steinbusch, H.W.; Andriessen, P., et al. Mesenchymal Stem Cells Induce T-Cell Tolerance and Protect the Preterm Brain after Global Hypoxia-Ischemia. PLOS ONE 2013, 8, e73031, doi:10.1371/journal.pone.0073031.

18. Ek, C.J.; D’Angelo, B.; Baburamani, A.A.; Lehner, C.; Leverin, A.L.; Smith, P.L.; Nilsson, H.; Svedin, P.; Hagberg, H.; Mallard, C. Brain barrier properties and cerebral blood flow in neonatal mice exposed to cerebral hypoxia-ischemia. J Cereb Blood Flow Metab 2015, 35, 818-827, doi:10.1038/jcbfm.2014.255.

19. Ophelders, D.R.; Wolfs, T.G.; Jellema, R.K.; Zwanenburg, A.; Andriessen, P.; Delhaas, T.; Ludwig, A.K.; Radtke, S.; Peters, V.; Janssen, L., et al. Mesenchymal Stromal Cell-Derived Extracellular Vesicles Protect the Fetal Brain After Hypoxia-Ischemia. Stem Cells Transl Med 2016, 5, 754-763, doi:10.5966/sctm.2015-0197.

20. Cristante, E.; McArthur, S.; Mauro, C.; Maggioli, E.; Romero, I.A.; Wylezinska-Arridge, M.; Couraud, P.O.; Lopez-Tremoleda, J.; Christian, H.C.; Weksler, B.B., et al. Identification of an essential endogenous regulator of blood-brain barrier integrity, and its pathological and therapeutic implications. Proc Natl Acad Sci U S A 2013, 110, 832-841, doi:10.1073/ pnas.1209362110.

21. Perretti, M.; D'Acquisto, F. Annexin A1 and glucocorticoids as effectors of the resolution of inflammation. Nature Reviews Immunology 2009, 9, 62, doi:10.1038/nri2470. 
22. Chen, K.; Bao, Z.; Gong, W.; Tang, P.; Yoshimura, T.; Wang, J.M. Regulation of inflammation by members of the formyl-peptide receptor family. Journal of Autoimmunity 2017, 85, 64-77, doi:https://doi.org/10.1016/j.jaut.2017.06.012.

23. He, H.Q.; Ye, R.D. The Formyl Peptide Receptors: Diversity of Ligands and Mechanism for Recognition. Molecules 2017, 22, doi:10.3390/molecules22030455.

24. Persidsky, Y.; Ramirez, S.H.; Haorah, J.; Kanmogne, G.D. Blood-brain barrier: structural components and function under physiologic and pathologic conditions. J Neuroimmune Pharmacol 2006, 1, 223-236, doi:10.1007/s11481-006-9025-3.

25. McArthur, S.; Cristante, E.; Paterno, M.; Christian, H.; Roncaroli, F.; Gillies, G.E.; Solito, E. Annexin A1: A Central Player in the Anti-Inflammatory and Neuroprotective Role of Microglia. Journal of immunology (Baltimore, Md. : 1950) 2010, 185, 6317-6328, doi:10.4049/jimmunol.1001095.

26. Probst-Cousin, S.; Kowolik, D.; Kuchelmeister, K.; Kayser, C.; Neundörfer, B.; Heuss, D. Expression of annexin-1 in multiple sclerosis plaques. Neuropathology and Applied Neurobiology 2002, 28, 292-300, doi:10.1046/j.1365-2990.2002.00396.x.

27. Knott, C.; Stern, G.; Wilkin, G.P. Inflammatory regulators in Parkinson's disease: iNOS, lipocortin-1, and cyclooxygenases-1 and -2. Mol Cell Neurosci 2000, 16, 724-739, doi:10.1006/mcne.2000.0914.

28. Maia, L.; de Moraes, C.N.; Dias, M.C.; Martinez, J.B.; Caballol, A.O.; Testoni, G.; de Queiroz, C.M.; Peña, R.D.; Landim-Alvarenga, F.C.; de Oliveira, E. A proteomic study of mesenchymal stem cells from equine umbilical cord. Theriogenology 2017, 100, 8-15, doi:https://doi.org/10.1016/j.theriogenology.2017.05.015.

29. de Moraes, C.N.; Maia, L.; de Oliveira, E.; de Paula Freitas Dell'Aqua, C.; Chapwanya, A.; da Cruz Landim-Alvarenga, F.; Oba, E. Shotgun proteomic analysis of the secretome of bovine endometrial mesenchymal progenitor/stem cells challenged or not with bacterial lipopolysaccharide. Veterinary Immunology and Immunopathology 2017, 187, 42-47, doi:https://doi.org/10.1016/j.vetimm.2017.03.007.

30. Rackham, C.L.; Vargas, A.E.; Hawkes, R.G.; Amisten, S.; Persaud, S.J.; Austin, A.L.; King, A.J.; Jones, P.M. Annexin A1 Is a Key Modulator of Mesenchymal Stromal CellMediated Improvements in Islet Function. Diabetes 2016, 65, 129-139, doi:10.2337/ db15-0990.

31. Srinivasan, B.; Kolli, A.R.; Esch, M.B.; Abaci, H.E.; Shuler, M.L.; Hickman, J.J. TEER measurement techniques for in vitro barrier model systems. J Lab Autom 2015, 20, 107-126, doi:10.1177/2211068214561025.

32. Back, S.A.; Riddle, A.; Hohimer, A.R. Role of instrumented fetal sheep preparations in defining the pathogenesis of human periventricular white-matter injury. Journal of child neurology 2006, 21, 582-589, doi:10.1177/08830738060210070101.

33. Kordelas, L.; Rebmann, V.; Ludwig, A.K.; Radtke, S.; Ruesing, J.; Doeppner, T.R.; Epple, M.; Horn, P.A.; Beelen, D.W.; Giebel, B. MSC-derived exosomes: a novel tool to treat therapyrefractory graft-versus-host disease. Leukemia 2014, 28, 970-973, doi:10.1038/leu.2014.41. 
34. Sokolova, V.; Ludwig, A.-K.; Hornung, S.; Rotan, O.; Horn, P.A.; Epple, M.; Giebel, B. Characterisation of exosomes derived from human cells by nanoparticle tracking analysis and scanning electron microscopy. Colloids and Surfaces B: Biointerfaces 2011, 87, 146-150, doi:https://doi.org/10.1016/j.colsurfb.2011.05.013.

35. Kusters, D.H.M.; Chatrou, M.L.; Willems, B.A.G.; De Saint-Hubert, M.; Bauwens, M.; van der Vorst, E.; Bena, S.; Biessen, E.A.L.; Perretti, M.; Schurgers, L.J., et al. Pharmacological Treatment with Annexin A1 Reduces Atherosclerotic Plaque Burden in LDLR-/- Mice on Western Type Diet. PLOS ONE 2015, 10, e0130484, doi:10.1371/journal.pone.0130484.

36. Bernas, M.J.; Cardoso, F.L.; Daley, S.K.; Weinand, M.E.; Campos, A.R.; Ferreira, A.J.; Hoying, J.B.; Witte, M.H.; Brites, D.; Persidsky, Y., et al. Establishment of primary cultures of human brain microvascular endothelial cells to provide an in vitro cellular model of the blood-brain barrier. Nat Protoc 2010, 5, 1265-1272, doi:10.1038/nprot.2010.76.

37. Kinney, H.C.; Volpe, J.J. Modeling the encephalopathy of prematurity in animals: the important role of translational research. Neurol Res Int 2012, 2012, 295389, doi:10.1155/2012/295389.

38. Galinsky, R.; Draghi, V.; Wassink, G.; Davidson, J.O.; Drury, P.P.; Lear, C.A.; Gunn, A.J.; Bennet, L. Magnesium sulfate reduces EEG activity but is not neuroprotective after asphyxia in preterm fetal sheep. J Cereb Blood Flow Metab 2017, 37, 1362-1373, doi:10.1177/0271678x16655548.

39. Wassink, G.; Davidson, J.O.; Dhillon, S.K.; Fraser, M.; Galinsky, R.; Bennet, L.; Gunn, A.J. Partial white and grey matter protection with prolonged infusion of recombinant human erythropoietin after asphyxia in preterm fetal sheep. Journal of cerebral blood flow and metabolism : official journal of the International Society of Cerebral Blood Flow and Metabolism 2017, 37, 1080-1094, doi:10.1177/0271678X16650455.

40. Weiss, N.; Miller, F.; Cazaubon, S.; Couraud, P.O. The blood-brain barrier in brain homeostasis and neurological diseases. Biochim Biophys Acta 2009, 1788, 842-857, doi:10.1016/j.bbamem.2008.10.022.

41. Engelhardt, S.; Huang, S.-F.; Patkar, S.; Gassmann, M.; Ogunshola, O.O. Differential responses of blood-brain barrier associated cells to hypoxia and ischemia: a comparative study. Fluids and Barriers of the CNS 2015, 12, 4, doi:10.1186/2045-8118-12-4.

42. Nishioku, T.; Matsumoto, J.; Dohgu, S.; Sumi, N.; Miyao, K.; Takata, F.; Shuto, H.; Yamauchi, A.; Kataoka, Y. Tumor necrosis factor-alpha mediates the blood-brain barrier dysfunction induced by activated microglia in mouse brain microvascular endothelial cells. J Pharmacol Sci 2010, 112, 251-254, doi:10.1254/jphs.09292sc.

43. Ries, M.; Loiola, R.; Shah, U.N.; Gentleman, S.M.; Solito, E.; Sastre, M. The antiinflammatory Annexin A1 induces the clearance and degradation of the amyloid-beta peptide. J Neuroinflammation 2016, 13, 234, doi:10.1186/s12974-016-0692-6.

44. Tang, G.; Liu, Y.; Zhang, Z.; Lu, Y.; Wang, Y.; Huang, J.; Li, Y.; Chen, X.; Gu, X.; Wang, Y., et al. Mesenchymal stem cells maintain blood-brain barrier integrity by inhibiting aquaporin-4 upregulation after cerebral ischemia. Stem Cells 2014, 32, 3150-3162, doi:10.1002/stem.1808. 
45. Bedi, S.S.; Aertker, B.M.; Liao, G.P.; Caplan, H.W.; Bhattarai, D.; Mandy, F.; Mandy, F.; Fernandez, L.G.; Zelnick, P.; Mitchell, M.B., et al. Therapeutic time window of multipotent adult progenitor therapy after traumatic brain injury. J Neuroinflammation 2018, 15, 84, doi:10.1186/s12974-018-1122-8.

46. Wang, Z.; Chen, Z.; Yang, J.; Yang, Z.; Yin, J.; Zuo, G.; Duan, X.; Shen, H.; Li, H.; Chen, G. Identification of two phosphorylation sites essential for annexin A1 in blood-brain barrier protection after experimental intracerebral hemorrhage in rats. Journal of cerebral blood flow and metabolism : official journal of the International Society of Cerebral Blood Flow and Metabolism 2017, 37, 2509-2525, doi:10.1177/0271678X16669513.

47. Sheikh, M.H.; Solito, E. Annexin A1: Uncovering the Many Talents of an Old Protein. International Journal of Molecular Sciences 2018, 19, 1045, doi:10.3390/ijms19041045.

48. Park, J.C.; Baik, S.H.; Han, S.H.; Cho, H.J.; Choi, H.; Kim, H.J.; Choi, H.; Lee, W.; Kim, D.K.; Mook-Jung, I. Annexin A1 restores A $\beta(1-42)$-induced blood-brain barrier disruption through the inhibition of RhoA-ROCK signaling pathway. Aging Cell 2017, 16, 149-161, doi:10.1111/acel.12530.

49. Maggioli, E.; McArthur, S.; Mauro, C.; Kieswich, J.; Kusters, D.H.; Reutelingsperger, C.P.; Yaqoob, M.; Solito, E. Estrogen protects the blood-brain barrier from inflammationinduced disruption and increased lymphocyte trafficking. Brain Behav Immun 2016, 51, 212-222, doi:10.1016/j.bbi.2015.08.020.

50. Johnston, M.V.; Hagberg, H. Sex and the pathogenesis of cerebral palsy. Developmental medicine and child neurology 2007, 49, 74-78, doi:10.1111/j.1469-8749.2007.0199a.x.

51. Mayoral, S.R.; Omar, G.; Penn, A.A. Sex differences in a hypoxia model of preterm brain damage. Pediatr Res 2009, 66, 248-253, doi:10.1203/PDR.0b013e3181b1bc34.

52. Al Mamun, A.; Yu, H.; Romana, S.; Liu, F. Inflammatory Responses are Sex Specific in Chronic Hypoxic-Ischemic Encephalopathy. Cell transplantation 2018, 27, 1328-1339, doi:10.1177/0963689718766362.

53. Morris, J.; Christian, H.; Flower, R. 19: Annexin 1 distribution in the CNS: an association with stem cells? Journal of Anatomy 2004, 205, 525-525.

54. Solito, E.; McArthur, S.; Christian, H.; Gavins, F.; Buckingham, J.C.; Gillies, G.E. Annexin A1 in the brain - undiscovered roles? Trends in Pharmacological Sciences 2008, 29, 135-142, doi:https://doi.org/10.1016/j.tips.2007.12.003.

55. Saunders, N.R.; Liddelow, S.A.; Dziegielewska, K.M. Barrier mechanisms in the developing brain. Frontiers in pharmacology 2012, 3, 46, doi:10.3389/fphar.2012.00046.

56. Whish, S.; Dziegielewska, K.M.; Mollgard, K.; Noor, N.M.; Liddelow, S.A.; Habgood, M.D.; Richardson, S.J.; Saunders, N.R. The inner CSF-brain barrier: developmentally controlled access to the brain via intercellular junctions. Front Neurosci 2015, 9, 16, doi:10.3389/fnins.2015.00016.

57. Fossan, G.; Cavanagh, M.; Evans, C.; Malinowska, D.; Møllgård, K.; Reynolds, M.; Saunders, N.J.D.B.R. CSF-brain permeability in the immature sheep fetus: a CSF-brain barrier. 1985, 18, 113-124. 
58. Liu, J.H.; Feng, D.; Zhang, Y.F.; Shang, Y.; Wu, Y.; Li, X.F.; Pei, L. Chloral Hydrate Preconditioning Protects Against Ischemic Stroke via Upregulating Annexin A1. CNS Neurosci Ther 2015, 21, 718-726, doi:10.1111/cns.12435.

59. Locatelli, I.; Sutti, S.; Jindal, A.; Vacchiano, M.; Bozzola, C.; Reutelingsperger, C.; Kusters, D.; Bena, S.; Parola, M.; Paternostro, C., et al. Endogenous annexin A1 is a novel protective determinant in nonalcoholic steatohepatitis in mice. Hepatology (Baltimore, Md.) 2014, 60, 531-544, doi:10.1002/hep.27141.

60. Luo, Z.Z.; Gao, Y.; Sun, N.; Zhao, Y.; Wang, J.; Tian, B.; Shi, J. Enhancing the interaction between annexin-1 and formyl peptide receptors regulates microglial activation to protect neurons from ischemia-like injury. Journal of Neuroimmunology 2014, 276, 24-36, doi:https://doi.org/10.1016/j.jneuroim.2014.07.013.

61. McArthur, S.; Gobbetti, T.; Juban, G.; Desgeorges, T.; Theret, M.; Gondin, J.; TollerKawahisa, J.; Reutelingsperger, C.; Perretti, M.; Mounier, R. Annexin A1 drives macrophage skewing towards a resolving phenotype to accelerate the regeneration of muscle injury through AMPK activation. bioRxiv 2018.

62. Plendl, J.; Neumüller, C.; Vollmar, A.; Auerbach, R.; Sinowatz, F. Isolation and characterization of endothelial cells from different organs of fetal pigs. Anatomy and Embryology 1996, 194, 445-456, doi:10.1007/BF00185992.

63. Plendl, J.; Sinowatz, F. Fetal versus adult brain endothelium in vitro: Characterization and reaction to tumor-conditioned media; 1995; Vol. 1, pp. 28-39.

64. Takata, F.; Dohgu, S.; Yamauchi, A.; Matsumoto, J.; Machida, T.; Fujishita, K.; Shibata, K.; Shinozaki, Y.; Sato, K.; Kataoka, Y., et al. In Vitro Blood-Brain Barrier Models Using Brain Capillary Endothelial Cells Isolated from Neonatal and Adult Rats Retain Age-Related Barrier Properties. PLOS ONE 2013, 8, e55166, doi:10.1371/journal. pone.0055166. 
$\underline{6}$ 


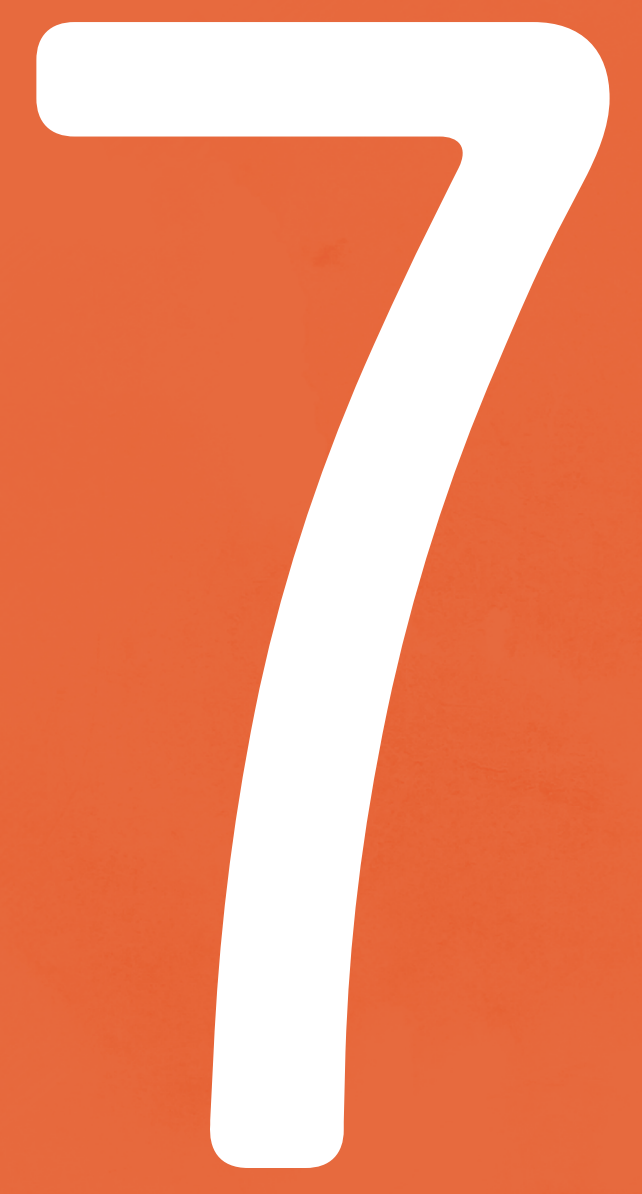

\section{Summary \& \\ general \\ discussion}





\section{Summary \& discussion}

Perinatal inflammatory stress and premature birth are strongly associated with neurological disabilities throughout life [1]. Over the last decades, extensive research has been performed to identify causes and unravel pathophysiological mechanisms, leading to injury of the developing brain in order to facilitate development of therapeutic strategies. Despite different approaches and numerous studies, many essential questions are still unanswered and the obtained knowledge resulted in only limited translation into clinical practice. As a result, still high rates of neurodevelopmental impairment are observed in survivors of prematurity. From the different essential questions that remain unanswered, I focused on the following items which formed the basis for the chapters in this thesis:

1. The peripheral and cerebral outcomes of the fetus following different intra amniotic microbial stimuli (chapter 2-4).

2. The time-dependent changes in the developing brain in the course of perinatal inflammatory stress (chapter $2 \& 3$ ).

3. The effect of repetitive inflammatory stimuli on preterm brain development (chapter 2).

4. Therapies for EoP with a focus on cell-based therapies and their regiondependent effects (chapter 5 \& 6).

5. Defining the optimal window of opportunity for new neuroprotective interventions (chapter $3 \& 6$ ).

Perinatal brain injury (EoP) can result from different origins with different underlying mechanisms. I, therefore, studied the effects of chorioamnionitis (chapter 2-4) and global hypoxia-ischemia (HI) (chapter 5-6) separately as two important, independent, contributing factors to EoP. For these studies, we have primarily used two preclinical ovine models closely mimicking human brain development in utero. In chapter 2-4, ovine models of intrauterine infection/inflammation have been used to mimic acute or chronic chorioamnionitis with fetal involvement (FIRS) to study its effects 
on fetal brain development. Considering the polymicrobial origin of chorioamnionitis different infectious/inflammatory triggers were used within these studies, including Ureaplasma Parvum, C. albicans and E. coli-derived Lipopolysaccharide (LPS) as representatives of most common bacterial and fungal isolates in chorioamnionitis [2].

In chapter 2-4 we showed that intra amniotic exposure to LPS or C. albicans provoked an acute increase in fetal systemic IL-6 concentrations within the first days after in utero exposure. More recently, these findings were recapitulated by UP (unpublished data), indicating that regardless of the microbial trigger a systemic inflammatory response is induced within the same timeframe. Pro-inflammatory cytokines, and IL-6 in particular, contribute to the activation of cerebral endothelium and cells of the blood-brain barrier [3-5]. These cells in turn activate adjacent microglia and astrocytes as their primary targets and producers of inflammatory mediators, ultimately resulting in a neuroinflammatory response [6-10]. This is also supported by our data in which we showed that the induction of a systemic response was followed by the induction of a cerebral inflammatory response as shown by an increase in microglia density and subsequently cerebral injury (chapter 2-4). In addition, IL-6 inhibits the development of new neurons in experimental models which is part of the pathogenesis of hyper-excitable neurological conditions including epilepsy and autism spectrum disorders $[3,11]$.

The clinical importance of increased fetal circulatory IL-6 levels has been further underscored by studies which showed a strong association between IL-6 levels and neonatal morbidity and mortality [12]. Numerous clinical studies have shown that fetal involvement by the induction of FIRS (characterized by increased pro-inflammatory cytokines including IL-6) during chorioamnionitis is related to higher rates of neonatal sepsis and multi-organ adverse outcomes including perinatal brain injury [12, 13] and in particular CP [14]. A recent study including 2,390 extremely preterm infants ( $<27$ weeks of gestation) demonstrated that the presence of clinical chorioamnionitis was associated with an increased risk of cognitive impairment at 18-22 months of corrected age [15]. In contrast, histological chorioamnionitis without fetal involvement has not been associated with adverse neurodevelopmental outcomes $[16,17]$ and shown to be only a weak risk factor for $\mathrm{CP}$ [16]. These combined data suggest that the release of inflammatory cytokines (FIRS) in the course of intrauterine infections 
play a crucial role in the initiation and aggravation of brain injury [14]. Based on the items described above, it is appealing to consider systemic IL-6 levels as a prognostic marker to predict cerebral outcomes. However, caution is required for the following reasons: Increased plasma IL-6 levels are also found after non-infection-related insults including Rh-alloimmunization [18], trauma [19] and HI insults [20]. It still needs to be established whether the prognosis of FIRS differs if induced by infectious or non-infectious insults. Moreover, increased plasma levels of IL-6 in the acute phase $(<24 \mathrm{~h})$ following an ischemic insult or traumatic brain injury have been shown to be a reliable prognostic marker for adverse cerebral outcomes $[21,22]$. We demonstrated that IL-6 concentrations were only acutely and transiently increased following intrauterine exposure to inflammation (UP, LPS) (chapter 2 and 3). Therefore, the timing of IL-6 analysis appears to be essential as marker for cerebral prognosis and needs to be incorporated into the clinical decision model. Further studies should focus on the use of IL-6 in combination with other implemented systemic inflammatory markers (CRP, procalcitonin) or novel biomarkers that can determine the onset of intra amniotic infections [23] to better predict cerebral outcomes. Currently, promising biomarker studies are ongoing that meet this need in the future.

In women who deliver preterm, Ureaplasma species are the most frequently isolated microorganisms from the amniotic fluid and placentae [24]. Although Ureaplasma parvum (UP) is known as a pathogen of low virulence, it is associated with chorioamnionitis, spontaneous abortions/ miscarriages and neonatal respiratory diseases [25]. Despite the fact that these microorganisms have been routinely found within placentae of pregnancies with chorioamnionitis, the role of Ureaplasma species as a causative agent has not been irrefutably explained. The controversy surrounding the role of UP in disease is reinforced by the fact that not all women infected with Ureaplasma spp. develop chorioamnionitis [25, 26]. In chapter 2 we however show that Ureaplasma parvum (UP) is not a harmless commensal colonizing the amniotic fluid. Chronic intra amniotic exposure induced changes in the fetal brain that might explain the observed altered neurological outcomes at 2 years of age in children born following chorioamnionitis by UP [27]. We found that chronic UP exposure in the third trimester decreased the number of astrocytes which was associated with increased number of oligodendrocytes (OLs) and epigenetic changes in the cerebral white matter and hippocampus. In line, changes in astrocyte function or density are associated with altered neurological 
outcomes [28]. In particular, altered astrocyte protein expression (GFAP) and disrupted astrocyte maturation have been implicated in the pathogenesis of neurodevelopmental disorders such as autism and cerebral palsy. In addition, the increase of OL lineage cells, as seen following $42 \mathrm{~d}$ of UP exposure, might indicate replenishment of OLs upon initial loss in the acute phase following UP exposure. This combined with the loss of MBP suggests a maturation arrest of OL progenitor cells which is a key feature of white matter injury in preterms $[29,30]$.

To gain more insight in the time dependent changes of the white matter in these fetal brains we studied the effects of intra amniotic LPS exposure over time. We found increased numbers of apoptotic cells in the white matter which was followed by loss of (pre-) OLs at 15 days post LPS exposure (chapter 3). Such changes of the white matter predispose to the long-term adverse neurological outcomes in later life [30], which supports the developmental origin of health and disease $(\mathrm{DOHaD})$ hypothesis. Considering the clinical importance, several potential mechanisms responsible for this altered white matter development, were studied and described in both chapter 2 and 3. First, aberrant or excessive microgliosis following LPS exposure was found (chapter 3) with subsequent cell death and/or maturation arrest of OLs with detrimental effects for the immature brain. Second, the observed alterations in astrocyte expression (chapter 2) is proposed to induce altered OL maturation since astrocytes are important for the metabolic support of OLs and key in regulating glutamate homeostasis [31]. At this stage of fetal brain development, pre-OLs are typically present in relative high numbers, and these cells are particularly vulnerable to glutamate receptor induced injury. When astrocyte function is altered, OLs lose metabolic support, glutamate homeostasis is disturbed and pre-OLs are prone to glutamate induced injury [32].

Alternatively, inflammation-induced epigenetic changes during early developmentcancause substantial lasting neurodevelopmentalimpairments later in life $[33,34]$. The observed increase of the DNA methylation marker 5-mc (chapter 2), an important repressor of gene transcription [35], is indicative for such epigenetic changes which are already induced during pregnancy complicated by chorioamnionitis. These findings highlight that essential alterations can occur in the antenatal period, which might be prerequisite for disturbed maturation and differentiation of immature OLs with potential detrimental consequences in later life. Based on 
the data described in this thesis, longitudinal follow up studies in our pre-clinical models are ongoing in which the long term consequences of the pathophysiological processes imitated in the perinatal period will be studied causally. Such a longitudinal study enables us to study tertiary mechanisms of brain injury which include epigenetic changes and persistent inflammation [9]. These processes can persist for months or years after the initial insult in the perinatal period and are proposed to contribute to the prevention of endogenous repair and regeneration which contribute to the neuropathological substrate of EoP [9].

Chorioamnionitis is not limited to bacteria or bacterial products but also include viral and fungal species [2]. Although intra-amniotic fungal infections are rare, its consequences on fetal development are of enormous impact including a high mortality rate and severe neurological disabilities. We have chosen $C$. albicans infection since this is the most common fungal species found in women diagnosed with chorioamnionitis (chapter 4) [2]. As shown in this chapter, intra-amniotic exposure to $C$. albicans resulted in a systemic immune response (increased IL-6 levels) with concomitant microglial and astrocyte activation, focal white matter disturbances, increased cell death and fetal death within 5 days upon exposure without fungal invasion of the brain parenchyma. We have previously shown that C. albicans resulted in skin, lung [36] and gastro-intestinal tract inflammation [37]. Moreover, Nikiforou et al. [37] showed fungal translocation through the epithelial barrier within the blood stream from 3 days onwards. Since at this stage no invasive growth of $C$. albicans was present in other organs including the brain parenchyma, we consider that the systemic inflammatory response at this stage of the infection as most important trigger for cerebral inflammation and subsequent injury which corresponds with the postulated pathophysiological changes of the brain following intra-amniotic UP and LPS exposure.

Antenatal treatment for Candida chorioamnionitis is challenging and, until now, only resulted in half of cases in the delivery of living infants [38]. We showed that intra-amniotic Fluconazole treatment after intraamniotic $C$. albicans infection successfully eradicated C. albicans from the CSF and temporarily inhibited systemic immune activation. However, modulation of the cerebral inflammatory response and prevention of the concomitant white matter injury was not observed. Nonetheless, a single dose of Fluconazole promoted fetal survival. In addition, Nikiforou et al. 
showed that Fluconazole treatment was successful in decreasing colonization and epithelial injury of the fetal gut. Taken together, we consider that the decreased mortality is a result of the decreased colonization and epithelial injury of the fetal gut and potentially the lung resulting in a temporarily diminished systemic inflammatory response. Besides eradication of the microbe, future studies should aim at preventing the inflammation-and/or HI-induced adverse effects on the brain including immune-modulatory/regenerative therapies.

The etiology of EoP is complex and multifactorial. Over the last decade (pre)clinical evidence demonstrated that the brain experiences altered susceptibility when exposed to a second injurious hit following pre-exposure to inflammation [39,40]. This concept of sensitization and preconditioning is supported by clinical data showing that the combination of antenatal infection and a hypoxic-ischemic (HI) insult around birth dramatically increases the risk of cerebral palsy (OR 78) when compared to either HI (OR 2.5) or infection (OR 7.2) alone [39]. Besides this specific combination of insults, multiple ante-, peri- or postnatal hits can contribute to the development of brain injury in the preterm infant including small for gestational age and impaired placental growth [41-43]. In addition, evidence is accumulating that postnatal ventilation-induced white matter injury, barotrauma around birth, (par)enteral feeding, necessary medication (glucocorticoids), surgeries, all could contribute to the development of EoP [44]. Clinically, exposure to multiple hits is associated with an enormous increase in the risk of and severity of white matter abnormalities [41, 45, 46]. Therefore, the impact of these factors on brain development also depends on interactions between different insults and treatments which need to be further explored.

In chapter 2 we investigated the effect of multiple inflammatory hits, representing intrauterine inflammation and possible postnatal infections, and the effect on brain development. In this double-hit study, we found that chronic intra-amniotic UP exposure prevented microgliosis, epigenetic and lipid profile changes and myelin disturbances when animals were exposed to a second inflammatory hit with lipopolysaccharide (LPS) from E.coli. In line, this 'preconditioning' phenotype was also found in ovine fetuses exposed to 70 days of UP and 7 days LPS [47]. Such preconditioning renders the brain less susceptible to a second insult, thereby resulting in less brain injury [48]. In contrast to these cerebral findings, UP infection for 24 days appeared to sensitize the fetal lungs [49] and gut (unpublished data) against a 
second inflammatory hit with LPS indicating that target organ and duration of the insult determines susceptibility for a second hit. The underlying mechanisms by which inflammation establishes a favorable environment protecting the brain against a second event remain largely unknown. Gene ontology analysis reveals that the most over-represented genes belong to immune and inflammatory processes and cell death pathways in LPS-induced preconditioning in the immature brain [48]. Moreover, the time delay between insults appears to be critical in determining the pathological outcome as the same sublethal exposure can render the tissue either more (sensitization) or less (preconditioning) sensitive to the same subsequent, severe insult, depending on the interval between events. Mallard et al. showed that the vulnerability of the brain to an HI insult following in utero inflammation differs between neonatal and adult brains resulting in sensitization and preconditioning respectively [50]. Altogether, these data support the complexity of the underlying pathophysiological mechanisms of EoP. Considering the clinical heterogeneity between patients makes it almost impossible to develop tailor-made treatments and predict outcomes on individual levels. Recognition of individual causal factors leading to brain damage is the first step in a complex translational mission to tailor safe and effective therapies resulting in individualized/ personalized medicine.

\section{Treatments}

The introduction of therapeutic hypothermia as a treatment for moderate to severe neonatal encephalopathy follows two decades of pre-clinical studies in experimental HI models and clinical trials [51]. There is clear evidence that therapeutic hypothermia in this setting reduces adverse outcomes including decrease of mortality and neurodevelopmental disability at 18 months of age (RR 0.75\% CI 0.68-0.83) which persists into childhood. However, around $40 \%$ of infants, have an adverse neurodevelopmental outcome, despite treatment [52]. Moreover, in preterm infants hypothermia is contra-indicated since it is associated with increased risk of side effects including poor neurodevelopmental outcome. Recently, the crucial role of the immune response has been recognized as having an important influence on outcome of hypothermia treatment [53-55]. It has been shown 
that therapeutic hypothermia is ineffective and even harmful in the presence of infection/inflammation in adult clinical studies [55]. In a pre-clinical neonatal rodent study cooling was not neuroprotective in inflammation-sensitized HI [54]. In a small prospective study of placental histology being related to MRI of babies undergoing therapeutic hypothermia, therapeutic hypothermia was less protective in babies whose placentas showed chorioamnionitis [55]. Considering these data, the need for new therapeutic strategies to prevent EoP is emerging. As already elaborated in the introduction of this thesis, stem cell therapies are of increasing interest as a neurotherapeutic for neonatal brain injury and may show great potential in the prevention of encephalopathy of prematurity [56]. As shown in chapter 2-4 the initiation of a cerebral inflammatory response is one of the essential steps leading to brain injury. Modulating this inflammatory response directly or indirectly is considered to be a key mechanism of action of a potential treatment. Second, regeneration of injured OLs and neurons and/or stimulate the maturation of these cells is a prerequisite of a new treatment to prevent EoP [9, 57]. Mesenchymal stromal cells possess both anti-inflammatory and regenerative properties. We have previously shown in an ovine model of HI-induced brain injury that intravenous administration of bone marrow-derived adherent stromal cells (MSCs, MAPCs) prevented cerebral inflammation, white matter injury and loss of function/decreased seizure activity following global hypoxiaischemia $[58,59]$.

Besides the cerebrum, the cerebellum is increasingly appreciated as an important contributor in EoP [60]. Clinical data show that disturbed cerebellar development in prematurely born children can still be detected at school age/adolescence and is associated with adverse neurodevelopmental outcome [61]. More precisely, it has been shown that cerebellar injury plays an important role in the high prevalence of non-motor deficits like cognition, learning and behavior in survivors of prematurity. Therefore, protection of the cerebellum following preterm birth and/or $\mathrm{HI}$ is clinically highly relevant. In chapter 5 we demonstrated that besides the cerebral white matter, MAPC therapy also protected the cerebellum against HI-induced injury. We found marked cortical injury, microgliosis and hypomyelination in the cerebellum following global $\mathrm{HI}$, changes that are indicative as a pre-stage for cerebellar underdevelopment [60]. We showed that these cerebellar alterations were prevented by intravenous administration of MAPCs and potentially may prevent neurodevelopmental disorders in later life. 
The different phases leading to brain injury following a $\mathrm{HI}$ insult have been well described and discussed in the introduction of this thesis [9, 62, 63]. Numerous studies have shown that microglia become excessively activated within 24 hours after an acute global HI insult and abundantly release pro-inflammatory cytokines, free oxygen species and excitatory amino acids [62-65]. Moreover, systemically, global HI also provokes a pro-inflammatory environment which triggers systemic release of danger signals reaching peak values around 24 hours after the insult [62-64, 66]. This systemic accumulation of DAMPs has been associated with massive activation of the peripheral immune system with rapid mobilization of immune effector cells (i.e. neutrophils, monocytes, T-cells) from the spleen [65, 67]. These mobilized effector cells can invade the neonatal brain through a disrupted blood-brain barrier and aggravate the existing injury [65,67]. This concept has been confirmed and extended by our data in a preclinical model of neonatal HIE, showing that neuroinflammation mediated by microglia was associated with marked mobilization of the peripheral immune system and splenic involution [58]. These immunomodulatory changes were associated with an increased seizure burden and induction of white matter injury. In our studies, we administered MSC [58] and MAPC (chapter 5) at $1 \mathrm{~h}$ and/or 4 days after global HI since these cells have shown to possess both strong anti-inflammatory and regenerative capacities in CNS injury. These capacities are influenced by the host' microenvironment. With the first dose, we aimed to dampen the acute peripheral and cerebral inflammatory response which is considered as one of the first steps leading to EoP [1, 9]. Interestingly, we showed in our pre-clinical model that MSC induced persistent peripheral T-cell tolerance in vivo and reduced invasion of T-cells into the preterm brain following global HI, which might be a mechanism underlying the therapeutic effects of MSCs [58]. Ultimately, attenuation of the cerebral and peripheral inflammatory response, will prevent/inhibit subsequent cell death and injury in the brain.

Besides the immunomodulatory effects, we postulate that MAPC cell therapy promotes remyelination in a similar manner as MSCs, which have been shown to stimulate neural progenitor cells to differentiate towards the OL lineage and induce remyelination in vivo. Therefore, the second dose was administered 4 days after global $\mathrm{HI}$ to support repair of oligodendrocyte and neuronal injury, which is initiated in the sub-acute phase after HI-injury. We showed that MAPC cells prevented hypomyelination and 
induced almost complete protection against PC loss at 7 days after HI. This is a very short period which makes protection a more likely mechanism than regeneration. In line, another group that studied the neuroprotective effects of stem cell based therapies in the preterm ovine fetus showed that these cells suppressed cerebral inflammation and protected white matter structures [68]. This study attributed these protective effects by indirect systemic and neuroimmunomodulatory effects. The neuroimmunomodulatory effects included the induction of a microglia phenotype switch towards a resting state, i.e. from a pro- inflammatory M1 phenotype to an anti-inflammatory M2 phenotype. Miron et al. showed that these M2 microglia enhance OL differentiation during remyelination in the brain [69]. Unfortunately, we cannot confirm the MAPC-induced switch towards a dominant, neuroprotective M2 phenotype since ovinespecific reagents to discriminate M1 from M2 microglia were not available at that moment. Recently, M2 specific markers (including CD 163) have been validated in fetal sheep studies which in future studies are useful to discriminate microglia phenotypes (unpublished). Altogether, we consider that especially theearly administration of MAPC cell treatment is accountable for the neuroprotective effects observed in this study. Although one might question the clinical feasibility of the 1 hour gift, recent experimental and clinical data indicated that later (12-36 hour) administration of stem cells similarly protected the adult brain after stroke [70]. Based on these combined findings, we expect that administration of MAPC cells to neonates at a clinically more feasible time point ( 24 hours) after the HI event will result in comparable neuroprotective effects as shown in our current study. Clinically, the pathophysiological process of encephalopathy in preterm infants evolves over time and therefore we cannot exclude that late administration of MAPCs would still exert beneficial effects at a later stage of brain development. Therefore, we consider the timing of analysis as an important limitation for the assessment of regenerative effects of MAPC cells in our study.

Despite that immunomodulatory and regenerative effects have been shown, the underlying mechanism of action of stem cell therapies remain largely unknown. It was initially thought that the therapeutic action of stem cells relied on direct replacement of dead and injured cells. However, since the number of cells that reach the site of injury is minimal, there is marginal engraftment and short cell survival $(72 \mathrm{~h})$, this theory was largely disregarded [59, 71]. In line, we and others could not identify MAPC cells 
within the cerebral parenchyma after 7 days [59, 71]. Meanwhile, there was growing evidence that the pharmacological effects of stem cell therapy rely at least in part on paracrine mechanisms since studies showed comparable therapeutic effects of MSC-conditioned medium when compared to its cellular equivalent $[72,73]$. Consistently, we have previously shown in our fetal sheep model of global HI that i.v. administration of mesenchymal stem cell-derived extracellular vesicles (MSC-EVs) resulted in partial protection against hypomyelination [74]. Remarkably, these therapeutic effects could not be explained by anti-inflammatory effects of the MSC-EVs such as seen after MSC treatment. This prompted us to focus on an alternative explanation for pharmacological effects of MSC-EVs, being restoring the injured BBB following global HI. There is accumulating evidence that the BBB around the second trimester becomes functional [75]. However, a global $\mathrm{HI}$ insult results in release of reactive oxygen species and excitotoxic molecules (direct/metabolic effects of $\mathrm{HI}$ ) and cytokines released from the peripheral and local innate immune system (inflammatory component) leading to BBB dysfunction [76]. An increased permeability of the BBB results in infiltration of peripheral immune cells (e.g. macrophages, leukocytes, T-cells) that can aggravate white matter injury by the release of pro-inflammatory mediators. Therefore, strengthening or restoring maintenance of BBB integrity by enforcing endothelial cells could restrict the extent of white matter injury. In chapter 6, we observed that global $\mathrm{HI}$ negatively affects the integrity of the fetal blood-brain barrier (BBB). We provide substantial evidence that this reduced integrity is associated with an acute drop in Annexin A1 (ANXA1) expression in the cerebral vasculature. Annexin A1 is known as an endogenous regulator of BBB integrity in neurodegenerative diseases [77]. Moreover, using an in vitro model of fetal cerebral endothelial cells we demonstrated that targeting the ANXA1/FPR axis is effective in restoring the BBB integrity loss following oxygen and glucose deprivation. Importantly, Annexins, including ANXA1, are frequently found in the proteome of stem cells and their EVs [78]. Altogether, we conclude from our previous and current findings that the protective effects of MSC-EVs arise, at least in part, from their protective actions on the BBB which may be mediated by Annexin A1. This concept is supported by a recent study that demonstrated that the BBB integrity in a murine brain endothelial cell line was rescued by administration of human recombinant ANXA1 following $\beta$-Amyloid 1-42 (A $\beta 42)$-induced BBB disruption [79]. Although our combined findings indicate that ANXA1driven inhibition of cerebral inflammation is not a plausible explanation 
for the pharmacological effects of MSC-EVs, we cannot rule out the possibility that microglia are part of the underlying working mechanism. More precisely, Solito et al. found that ANXA1 plays an important role in controlling non-inflammatory phagocytosis of apoptotic cells and promoting resolution of inflammation in models of Alzheimer's disease [80]. An Annexin-driven switch into M2 microglia that typically express such characteristics is a realistic scenario that warrants further investigation in a follow up study where neuroprotective effects of ANXA1 will be directly tested in vivo. Taken together, we and others show that the beneficial effects of stromal derived cells depend on a combination of actions of the intact cell and its secretome.

\section{Future studies - personalized medicine - designing targeted stem cell therapies}

The work presented in this thesis helps to unravel the time-dependent changes in the fetal brain following perinatal inflammatory stress. Apoptotic cell death, measured by the number of cleaved caspase- 3 positive cells, is an important prognostic factor for neurological outcomes [81]. Importantly, whereas HI-induced cell death has been observed within 3 days after the HI insult [82, 83], we found increased apoptotic cell death following antenatal exposure to infection from 8 days post intra-amniotic LPS exposure (chapter 3). These time dependent differences in the acute phase following a sterile or infectious inflammatory trigger are important determinants in defining a treatment regimen.

The data presented in this thesis indicate that defined windows of opportunities emerge following perinatal inflammatory triggers which are different following an $\mathrm{HI}$ insult compared to an infectious trigger. These important findings indicate a potential mismatch between the optimal timing and current clinical initiation of treatment regimens which might be an explanation for the reported inconsistencies of clinical trials. This may in particular be true for trials with EPO [84]. More precisely: the work 
described in chapter 3 \& 6 shows a time-dependent and transient decrease of the pEPOR expression and endogenous ANXA1 content in the brain. Therefore, availability of a technology to detect the onset of inflammation in utero in a safe and non-invasive manner is key for the application of this concept. Moreover, every child that is born has its own "fingerprint" of risk factors potentially contributing to the development of EoP. This demands detection tools for the recognition of these distinct risk factors and a more individualized treatment approach. Correct timing of treatment initiation in relation to the nature and stage of injury is of great clinical importance in the near future.

Our studies and other preclinical studies primarily focused on single hit models with no standardized route, dose or intervention time of (cellbased or cell-derived) therapies. Timing and route of delivery for many treatments and dosage related effects are likely to play a role in efficacy and therapeutic potential. In experimental settings, several research groups have used intracranial delivery of neurotherapeutics [85, 86], however clinically a less invasive administration route is preferred [87]. Intranasal delivery is emerging as an effective administration method for especially cell based therapies which directly targets the brain, preventing loss of cells in the peripheral organs. Comparing intravenous delivery, which has the proposed advantage of reducing systemic inflammation as a key factor in initiating EoP, to intranasal delivery should be elucidated in future studies [56].

The best studied treatment currently in clinical trials is EPO. Although EPO is an endogenous factor expressed in the fetal brain, preclinical studies have shown that EPO treatment requires pharmacological, non-physiological doses to cross the blood-brain barrier to acquire a neuroprotective effect $[88,89]$. Clinical trials still show great variability in used dosages of EPO which could explain the inconsistent results [84].

Until today, hypothermia is the only clinically available effective neuroprotective treatment for neonates at risk for brain injury. We know that hypothermia alters the time course of pathophysiologic events resulting in brain injury including energy failure and inflammation. Moreover, it modifies drug metabolism drastically. Therefore, designing new neurotherapeutic strategies must consider the pharmacological alterations induced by hypothermia and future studies should focus on these potential interactions of treatments. 
In conclusion, the nature of brain injury in preterm neonates is very complex, multi factorial and sensitive approaches for patient identification are essential. In addition, preclinical studies primarily focused on single hit models with no standardized route, dose or intervention time of (cell-based or cell-derived) therapies. This mismatch impedes development and or translation of neurological therapeutics for preterm infants, explaining the existing unmet clinical need. As such, future (pre) clinical studies should (1) address the multi-factorial nature of EoP, thereby allowing (2) proper patient stratification; (3) focus on dose, route and timing; and (4) explore synergistic approaches in which cell-based therapies are combined with therapies, such as EPO, melatonin, glucocorticoids, and hypothermia. Achieving the correct poise of interventions at the correct time in relation to the underlying pathophysiology and stage of injury will be a significant challenge in the next years. 


\section{References}

1. Volpe, J. J. "The Encephalopathy of Prematurity--Brain Injury and Impaired Brain Development Inextricably Intertwined." Semin Pediatr Neurol 16, no. 4 (2009): 167-78.

2. DiGiulio, D. B., R. Romero, H. P. Amogan, J. P. Kusanovic, E. M. Bik, F. Gotsch, C. J. Kim, O. Erez, S. Edwin, and D. A. Relman. "Microbial Prevalence, Diversity and Abundance in Amniotic Fluid During Preterm Labor: A Molecular and Culture-Based Investigation." PLoS One 3, no. 8 (2008): e3056.

3. Deverman, B. E., and P. H. Patterson. "Cytokines and Cns Development." Neuron 64, no. 1 (2009): 61-78.

4. Dammann, O., and T. M. O'Shea. “Cytokines and Perinatal Brain Damage." Clin Perinatol 35, no. 4 (2008): 643-63, v.

5. Rochfort, K. D., and P. M. Cummins. "The Blood-Brain Barrier Endothelium: A Target for Pro-Inflammatory Cytokines." Biochem Soc Trans 43, no. 4 (2015): 702-6.

6. Hagberg, H., C. Mallard, D. M. Ferriero, S. J. Vannucci, S. W. Levison, Z. S. Vexler, and P. Gressens. "The Role of Inflammation in Perinatal Brain Injury." Nat Rev Neurol 11, no. 4 (2015): 192-208.

7. Mallard, C., M. E. Tremblay, and Z. S. Vexler. "Microglia and Neonatal Brain Injury." Neuroscience 405 (2019): 68-76.

8. Van Steenwinckel, J., A. L. Schang, S. Sigaut, V. Chhor, V. Degos, H. Hagberg, O. Baud, B. Fleiss, and P. Gressens. "Brain Damage of the Preterm Infant: New Insights into the Role of Inflammation." Biochem Soc Trans 42, no. 2 (2014): 557-63.

9. Fleiss, B., and P. Gressens. "Tertiary Mechanisms of Brain Damage: A New Hope for Treatment of Cerebral Palsy?" Lancet Neurol 11, no. 6 (2012): 556-66.

10. Favrais, G., Y. van de Looij, B. Fleiss, N. Ramanantsoa, P. Bonnin, G. StoltenburgDidinger, A. Lacaud, E. Saliba, O. Dammann, J. Gallego, S. Sizonenko, H. Hagberg, V. Lelievre, and P. Gressens. "Systemic Inflammation Disrupts the Developmental Program of White Matter." Ann Neurol 70, no. 4 (2011): 550-65.

11. Atzori, M., F. Garcia-Oscos, and J. A. Mendez. "Role of Il-6 in the Etiology of Hyperexcitable Neuropsychiatric Conditions: Experimental Evidence and Therapeutic Implications." Future Med Chem 4, no. 17 (2012): 2177-92.

12. Gomez, R., R. Romero, F. Ghezzi, B. H. Yoon, M. Mazor, and S. M. Berry. "The Fetal Inflammatory Response Syndrome." Am J Obstet Gynecol 179, no. 1 (1998): 194-202.

13. Yoon, B. H., R. Romero, J. S. Park, M. Kim, S. Y. Oh, C. J. Kim, and J. K. Jun. “The Relationship among Inflammatory Lesions of the Umbilical Cord (Funisitis), Umbilical Cord Plasma Interleukin 6 Concentration, Amniotic Fluid Infection, and Neonatal Sepsis." Am J Obstet Gynecol 183, no. 5 (2000): 1124-9. 
14. Yoon, B. H., J. K. Jun, R. Romero, K. H. Park, R. Gomez, J. H. Choi, and I. O. Kim. "Amniotic Fluid Inflammatory Cytokines (Interleukin-6, Interleukin-1beta, and Tumor Necrosis Factor-Alpha), Neonatal Brain White Matter Lesions, and Cerebral Palsy." Am J Obstet Gynecol 177, no. 1 (1997): 19-26.

15. Pappas, A., D. E. Kendrick, S. Shankaran, B. J. Stoll, E. F. Bell, A. R. Laptook, M. C. Walsh, A. Das, E. C. Hale, N. S. Newman, R. D. Higgins, Health Eunice Kennedy Shriver National Institute of Child, and Network Human Development Neonatal Research. "Chorioamnionitis and Early Childhood Outcomes among Extremely Low-Gestational-Age Neonates." JAMA Pediatr 168, no. 2 (2014): 137-47.

16. Shi, Z., L. Ma, K. Luo, M. Bajaj, S. Chawla, G. Natarajan, H. Hagberg, and S. Tan. "Chorioamnionitis in the Development of Cerebral Palsy: A Meta-Analysis and Systematic Review." Pediatrics 139, no. 6 (2017).

17. Bierstone, D., N. Wagenaar, D. L. Gano, T. Guo, G. Georgio, F. Groenendaal, L. S. de Vries, J. Varghese, H. C. Glass, C. Chung, J. Terry, M. Rijpert, R. E. Grunau, A. Synnes, A. J. Barkovich, D. M. Ferriero, M. Benders, V. Chau, and S. P. Miller. "Association of Histologic Chorioamnionitis with Perinatal Brain Injury and Early Childhood Neurodevelopmental Outcomes among Preterm Neonates." JAMA Pediatr 172, no. 6 (2018): 534-41.

18. Vaisbuch, E., R. Romero, R. Gomez, J. P. Kusanovic, S. Mazaki-Tovi, T. Chaiworapongsa, and S. S. Hassan. "An Elevated Fetal Interleukin-6 Concentration Can Be Observed in Fetuses with Anemia Due to Rh Alloimmunization: Implications for the Understanding of the Fetal Inflammatory Response Syndrome." J Matern Fetal Neonatal Med 24, no. 3 (2011): 391-6.

19. Rodney, T., N. Osier, and J. Gill. "Pro- and Anti-Inflammatory Biomarkers and Traumatic Brain Injury Outcomes: A Review." Cytokine 110 (2018): 248-56.

20. Chiesa, C., G. Pellegrini, A. Panero, T. De Luca, M. Assumma, F. Signore, and L. Pacifico. "Umbilical Cord Interleukin-6 Levels Are Elevated in Term Neonates with Perinatal Asphyxia." Eur J Clin Invest 33, no. 4 (2003): 352-8.

21. Waje-Andreassen, U., J. Krakenes, E. Ulvestad, L. Thomassen, K. M. Myhr, J. Aarseth, and C. A. Vedeler. "Il-6: An Early Marker for Outcome in Acute Ischemic Stroke." Acta Neurol Scand 111, no. 6 (2005): 360-5.

22. Woiciechowsky, C., B. Schoning, J. Cobanov, W. R. Lanksch, H. D. Volk, and W. D. Docke. "Early Il-6 Plasma Concentrations Correlate with Severity of Brain Injury and Pneumonia in Brain-Injured Patients." J Trauma 52, no. 2 (2002): 339-45.

23. Woodcock, T., and M. C. Morganti-Kossmann. "The Role of Markers of Inflammation in Traumatic Brain Injury." Front Neurol 4 (2013): 18.

24. Goldenberg, R. L., J. C. Hauth, and W. W. Andrews. "Intrauterine Infection and Preterm Delivery." N Engl J Med 342, no. 20 (2000): 1500-7.

25. Sweeney, E. L., S. J. Dando, S. G. Kallapur, and C. L. Knox. “The Human Ureaplasma Species as Causative Agents of Chorioamnionitis." Clin Microbiol Rev 30, no. 1 (2017): 349-79. 
26. Donders, G. G. G., K. Ruban, G. Bellen, and L. Petricevic. "Mycoplasma/Ureaplasma Infection in Pregnancy: To Screen or Not to Screen." J Perinat Med 45, no. 5 (2017): 505-15.

27. Berger, A., A. Witt, N. Haiden, A. Kaider, K. Klebermasz, R. Fuiko, M. Langgartner, and A. Pollak. "Intrauterine Infection with Ureaplasma Species Is Associated with Adverse Neuromotor Outcome at 1 and 2 Years Adjusted Age in Preterm Infants." J Perinat Med 37, no. 1 (2009): 72-8.

28. Yang, Y., H. Higashimori, and L. Morel. "Developmental Maturation of Astrocytes and Pathogenesis of Neurodevelopmental Disorders." J Neurodev Disord 5, no. 1 (2013): 22.

29. Penn, A. A., P. Gressens, B. Fleiss, S. A. Back, and V. Gallo. "Controversies in Preterm Brain Injury." Neurobiol Dis 92, no. Pt A (2016): 90-101.

30. Back, S. A., and S. P. Miller. "Brain Injury in Premature Neonates: A Primary Cerebral Dysmaturation Disorder?" Ann Neurol 75, no. 4 (2014): 469-86.

31. Carson, M. J., J. C. Thrash, and B. Walter. "The Cellular Response in Neuroinflammation: The Role of Leukocytes, Microglia and Astrocytes in Neuronal Death and Survival." Clin Neurosci Res 6, no. 5 (2006): 237-45.

32. Volpe, J. J. "Neurobiology of Periventricular Leukomalacia in the Premature Infant." Pediatr Res 50, no. 5 (2001): 553-62.

33. Jirtle, R. L., and M. K. Skinner. "Environmental Epigenomics and Disease Susceptibility." Nat Rev Genet 8, no. 4 (2007): 253-62.

34. Bilbo, S. D., and J. M. Schwarz. "Early-Life Programming of Later-Life Brain and Behavior: A Critical Role for the Immune System." Front Behav Neurosci 3 (2009): 14.

35. Ficz, G., M. R. Branco, S. Seisenberger, F. Santos, F. Krueger, T. A. Hore, C. J. Marques, S. Andrews, and W. Reik. "Dynamic Regulation of 5-Hydroxymethylcytosine in Mouse Es Cells and During Differentiation." Nature 473, no. 7347 (2011): 398-402.

36. Maneenil, G., M. S. Payne, P. Senthamarai Kannan, S. G. Kallapur, B. W. Kramer, J. P. Newnham, Y. Miura, A. H. Jobe, and M. W. Kemp. "Fluconazole Treatment of Intrauterine Candida Albicans Infection in Fetal Sheep." Pediatr Res 77, no. 6 (2015): 740-8.

37. Nikiforou, M., E. M. Jacobs, M. W. Kemp, M. W. Hornef, M. S. Payne, M. Saito, J. P. Newnham, L. E. Janssen, A. H. Jobe, S. G. Kallapur, B. W. Kramer, and T. G. Wolfs. "Intra-Amniotic Candida Albicans Infection Induces Mucosal Injury and Inflammation in the Ovine Fetal Intestine." Sci Rep 6 (2016): 29806.

38. Maki, Y., M. Fujisaki, Y. Sato, and H. Sameshima. "Candida Chorioamnionitis Leads to Preterm Birth and Adverse Fetal-Neonatal Outcome." Infect Dis Obstet Gynecol 2017 (2017): 9060138.

39. Fleiss, B., C. J. Tann, V. Degos, S. Sigaut, J. Van Steenwinckel, A. L. Schang, A. Kichev, N. J. Robertson, C. Mallard, H. Hagberg, and P. Gressens. "Inflammation-Induced Sensitization of the Brain in Term Infants." Dev Med Child Neurol 57 Suppl 3 (2015): 17-28. 
40. Hagberg, H., O. Dammann, C. Mallard, and A. Leviton. "Preconditioning and the Developing Brain." Semin Perinatol 28, no. 6 (2004): 389-95.

41. Leviton, A., R. N. Fichorova, T. M. O'Shea, K. Kuban, N. Paneth, O. Dammann, E. N. Allred, and Elgan Study Investigators. "Two-Hit Model of Brain Damage in the Very Preterm Newborn: Small for Gestational Age and Postnatal Systemic Inflammation." Pediatr Res 73, no. 3 (2013): 362-70.

42. Khwaja, O., and J. J. Volpe. "Pathogenesis of Cerebral White Matter Injury of Prematurity." Arch Dis Child Fetal Neonatal Ed 93, no. 2 (2008): F153-61.

43. Miller, S. L., P. S. Huppi, and C. Mallard. "The Consequences of Fetal Growth Restriction on Brain Structure and Neurodevelopmental Outcome." J Physiol 594, no. 4 (2016): 807-23.

44. Barton, S. K., M. Tolcos, S. L. Miller, C. Christoph-Roehr, G. M. Schmolzer, T. J. Moss, S. B. Hooper, E. M. Wallace, and G. R. Polglase. "Ventilation-Induced Brain Injury in Preterm Neonates: A Review of Potential Therapies." Neonatology 110, no. 2 (2016): 155-62.

45. Korzeniewski, S. J., R. Romero, J. Cortez, A. Pappas, A. G. Schwartz, C. J. Kim, J. S. Kim, Y. M. Kim, B. H. Yoon, T. Chaiworapongsa, and S. S. Hassan. "A "Multi-Hit" Model of Neonatal White Matter Injury: Cumulative Contributions of Chronic Placental Inflammation, Acute Fetal Inflammation and Postnatal Inflammatory Events." J Perinat Med 42, no. 6 (2014): 731-43.

46. Barnett, M. L., N. Tusor, G. Ball, A. Chew, S. Falconer, P. Aljabar, J. A. Kimpton, N. Kennea, M. Rutherford, A. David Edwards, and S. J. Counsell. "Exploring the Multiple-Hit Hypothesis of Preterm White Matter Damage Using Diffusion Mri." Neuroimage Clin 17 (2018): 596-606.

47. Kallapur, S. G., B. W. Kramer, C. L. Knox, C. A. Berry, J. J. Collins, M. W. Kemp, I. Nitsos, G. R. Polglase, J. Robinson, N. H. Hillman, J. P. Newnham, C. Chougnet, and A. H. Jobe. "Chronic Fetal Exposure to Ureaplasma Parvum Suppresses Innate Immune Responses in Sheep." J Immunol 187, no. 5 (2011): 2688-95.

48. Mallard, C., and H. Hagberg. "Inflammation-Induced Preconditioning in the Immature Brain." Semin Fetal Neonatal Med 12, no. 4 (2007): 280-6.

49. Willems, M. G. M., M. W. Kemp, L. A. Fast, N. M. M. Wagemaker, L. E. W. Janssen, J. P. Newnham, M. S. Payne, O. B. Spiller, S. G. Kallapur, A. H. Jobe, T. Delhaas, B. W. Kramer, and Tgam Wolfs. "Pulmonary Vascular Changes in Extremely Preterm Sheep after Intra-Amniotic Exposure to Ureaplasma Parvum and Lipopolysaccharide." PLoS One 12, no. 6 (2017): e0180114.

50. Wang, X., H. Hagberg, C. Nie, C. Zhu, T. Ikeda, and C. Mallard. "Dual Role of Intrauterine Immune Challenge on Neonatal and Adult Brain Vulnerability to Hypoxia-Ischemia." J Neuropathol Exp Neurol 66, no. 6 (2007): 552-61.

51. Jacobs, S., R. Hunt, W. Tarnow-Mordi, T. Inder, and P. Davis. "Cooling for Newborns with Hypoxic Ischaemic Encephalopathy." Cochrane Database Syst Rev, no. 4 (2007): CD003311. 
52. Roka, A., and D. Azzopardi. "Therapeutic Hypothermia for Neonatal Hypoxic Ischaemic Encephalopathy." Early Hum Dev 86, no. 6 (2010): 361-7.

53. An, C., Y. Shi, P. Li, X. Hu, Y. Gan, R. A. Stetler, R. K. Leak, Y. Gao, B. L. Sun, P. Zheng, and J. Chen. "Molecular Dialogs between the Ischemic Brain and the Peripheral Immune System: Dualistic Roles in Injury and Repair." Prog Neurobiol 115 (2014): 6-24.

54. Osredkar, D., M. Thoresen, E. Maes, T. Flatebo, M. Elstad, and H. Sabir. “Hypothermia Is Not Neuroprotective after Infection-Sensitized Neonatal Hypoxic-Ischemic Brain Injury." Resuscitation 85, no. 4 (2014): 567-72.

55. Wintermark, P., T. Boyd, M. C. Gregas, M. Labrecque, and A. Hansen. "Placental Pathology in Asphyxiated Newborns Meeting the Criteria for Therapeutic Hypothermia." Am J Obstet Gynecol 203, no. 6 (2010): 579 e1-9.

56. Wagenaar, N., C. H. Nijboer, and F. van Bel. "Repair of Neonatal Brain Injury: Bringing Stem Cell-Based Therapy into Clinical Practice." Dev Med Child Neurol 59, no. 10 (2017): 997-1003.

57. Davidson, J. O., J. M. Dean, M. Fraser, G. Wassink, T. C. Andelius, S. K. Dhillon, L. Bennet, and A. J. Gunn. "Perinatal Brain Injury: Mechanisms and Therapeutic Approaches." Front Biosci (Landmark Ed) 23 (2018): 2204-26.

58. Jellema, R. K., T. G. Wolfs, V. Lima Passos, A. Zwanenburg, D. R. Ophelders, E. Kuypers, A. H. Hopman, J. Dudink, H. W. Steinbusch, P. Andriessen, W. T. Germeraad, J. Vanderlocht, and B. W. Kramer. "Mesenchymal Stem Cells Induce T-Cell Tolerance and Protect the Preterm Brain after Global Hypoxia-Ischemia." PLoS One 8, no. 8 (2013): e73031.

59. Jellema, R. K., D. R. Ophelders, A. Zwanenburg, M. Nikiforou, T. Delhaas, P. Andriessen, R. W. Mays, R. Deans, W. T. Germeraad, T. G. Wolfs, and B. W. Kramer. "Multipotent Adult Progenitor Cells for Hypoxic-Ischemic Injury in the Preterm Brain." J Neuroinflammation 12 (2015): 241.

60. Volpe, J. J. "Cerebellum of the Premature Infant: Rapidly Developing, Vulnerable, Clinically Important." J Child Neurol 24, no. 9 (2009): 1085-104.

61. Pieterman, K., T. J. White, G. E. van den Bosch, W. J. Niessen, I. K. M. Reiss, D. Tibboel, F. E. Hoebeek, and J. Dudink. "Cerebellar Growth Impairment Characterizes SchoolAged Children Born Preterm without Perinatal Brain Lesions." AJNR Am J Neuroradiol 39, no. 5 (2018): 956-62.

62. Wang, Q., H. Lv, L. Lu, P. Ren, and L. Li. “Neonatal Hypoxic-Ischemic Encephalopathy: Emerging Therapeutic Strategies Based on Pathophysiologic Phases of the Injury." J Matern Fetal Neonatal Med (2018): 1-8.

63. Nair, J., and V. H. S. Kumar. "Current and Emerging Therapies in the Management of Hypoxic Ischemic Encephalopathy in Neonates." Children (Basel) 5, no. 7 (2018).

64. Douglas-Escobar, M., and M. D. Weiss. “Hypoxic-Ischemic Encephalopathy: A Review for the Clinician." JAMA Pediatr 169, no. 4 (2015): 397-403. 
65. Lai, J. C. Y., E. Rocha-Ferreira, C. J. Ek, X. Wang, H. Hagberg, and C. Mallard. "Immune Responses in Perinatal Brain Injury." Brain Behav Immun 63 (2017): 210-23.

66. Jenkins, D. D., L. G. Rollins, J. K. Perkel, C. L. Wagner, L. P. Katikaneni, W. T. Bass, D. A. Kaufman, M. J. Horgan, S. Languani, L. Givelichian, K. Sankaran, J. Y. Yager, and R. H. Martin. "Serum Cytokines in a Clinical Trial of Hypothermia for Neonatal Hypoxic-Ischemic Encephalopathy." J Cereb Blood Flow Metab 32, no. 10 (2012): 1888-96.

67. Johnston, M. V., A. Fatemi, M. A. Wilson, and F. Northington. "Treatment Advances in Neonatal Neuroprotection and Neurointensive Care." Lancet Neurol 10, no. 4 (2011): 372-82.

68. Li, J., T. Yawno, A. Sutherland, J. Loose, I. Nitsos, R. Bischof, M. Castillo-Melendez, C. A. McDonald, F. Y. Wong, G. Jenkin, and S. L. Miller. "Preterm White Matter Brain Injury Is Prevented by Early Administration of Umbilical Cord Blood Cells." Exp Neurol 283, no. Pt A (2016): 179-87.

69. Miron, V. E., A. Boyd, J. W. Zhao, T. J. Yuen, J. M. Ruckh, J. L. Shadrach, P. van Wijngaarden, A. J. Wagers, A. Williams, R. J. M. Franklin, and C. Ffrench-Constant. "M2 Microglia and Macrophages Drive Oligodendrocyte Differentiation During Cns Remyelination." Nat Neurosci 16, no. 9 (2013): 1211-18.

70. Hess, D. C., L. R. Wechsler, W. M. Clark, S. I. Savitz, G. A. Ford, D. Chiu, D. R. Yavagal, K. Uchino, D. S. Liebeskind, A. P. Auchus, S. Sen, C. A. Sila, J. D. Vest, and R. W. Mays. "Safety and Efficacy of Multipotent Adult Progenitor Cells in Acute Ischaemic Stroke (Masters): A Randomised, Double-Blind, Placebo-Controlled, Phase 2 Trial." Lancet Neurol 16, no. 5 (2017): 360-68.

71. Yasuhara, T., K. Hara, M. Maki, R. W. Mays, R. J. Deans, D. C. Hess, J. E. Carroll, and C. V. Borlongan. "Intravenous Grafts Recapitulate the Neurorestoration Afforded by Intracerebrally Delivered Multipotent Adult Progenitor Cells in Neonatal HypoxicIschemic Rats." J Cereb Blood Flow Metab 28, no. 11 (2008): 1804-10.

72. Lai, R. C., T. S. Chen, and S. K. Lim. "Mesenchymal Stem Cell Exosome: A Novel Stem Cell-Based Therapy for Cardiovascular Disease." Regen Med 6, no. 4 (2011): 481-92.

73. Lai, R. C., F. Arslan, M. M. Lee, N. S. Sze, A. Choo, T. S. Chen, M. Salto-Tellez, L. Timmers, C. N. Lee, R. M. El Oakley, G. Pasterkamp, D. P. de Kleijn, and S. K. Lim. "Exosome Secreted by Msc Reduces Myocardial Ischemia/Reperfusion Injury." Stem Cell Res 4, no. 3 (2010): 214-22.

74. Ophelders, D. R., T. G. Wolfs, R. K. Jellema, A. Zwanenburg, P. Andriessen, T. Delhaas, A. K. Ludwig, S. Radtke, V. Peters, L. Janssen, B. Giebel, and B. W. Kramer. "Mesenchymal Stromal Cell-Derived Extracellular Vesicles Protect the Fetal Brain after Hypoxia-Ischemia." Stem Cells Transl Med 5, no. 6 (2016): 754-63.

75. Saunders, N. R., R. Daneman, K. M. Dziegielewska, and S. A. Liddelow. "Transporters of the Blood-Brain and Blood-Csf Interfaces in Development and in the Adult." Mol Aspects Med 34, no. 2-3 (2013): 742-52. 
76. Moretti, R., J. Pansiot, D. Bettati, N. Strazielle, J. F. Ghersi-Egea, G. Damante, B. Fleiss, L. Titomanlio, and P. Gressens. "Blood-Brain Barrier Dysfunction in Disorders of the Developing Brain." Front Neurosci 9 (2015): 40.

77. Cristante, E., S. McArthur, C. Mauro, E. Maggioli, I. A. Romero, M. Wylezinska-Arridge, P. O. Couraud, J. Lopez-Tremoleda, H. C. Christian, B. B. Weksler, A. Malaspina, and E. Solito. "Identification of an Essential Endogenous Regulator of Blood-Brain Barrier Integrity, and Its Pathological and Therapeutic Implications." Proc Natl Acad Sci U S A 110, no. 3 (2013): 832-41.

78. Maia, L., C. N. de Moraes, M. C. Dias, J. B. Martinez, A. O. Caballol, G. Testoni, C. M. de Queiroz, R. D. Pena, F. C. Landim-Alvarenga, and E. de Oliveira. "A Proteomic Study of Mesenchymal Stem Cells from Equine Umbilical Cord." Theriogenology 100 (2017): 8-15.

79. Park, J. C., S. H. Baik, S. H. Han, H. J. Cho, H. Choi, H. J. Kim, H. Choi, W. Lee, D. K. Kim, and I. Mook-Jung. "Annexin A1 Restores Abeta1-42 -Induced Blood-Brain Barrier Disruption through the Inhibition of Rhoa-Rock Signaling Pathway." Aging Cell 16, no. 1 (2017): 149-61.

80. McArthur, S., E. Cristante, M. Paterno, H. Christian, F. Roncaroli, G. E. Gillies, and E. Solito. "Annexin A1: A Central Player in the Anti-Inflammatory and Neuroprotective Role of Microglia." J Immunol 185, no. 10 (2010): 6317-28.

81. Rosell, A., E. Cuadrado, J. Alvarez-Sabin, M. Hernandez-Guillamon, P. Delgado, A. Penalba, M. Mendioroz, A. Rovira, I. Fernandez-Cadenas, M. Ribo, C. A. Molina, and J. Montaner. "Caspase-3 Is Related to Infarct Growth after Human Ischemic Stroke." Neurosci Lett 430, no. 1 (2008): 1-6.

82. Barrett, R. D., L. Bennet, A. Naylor, S. A. George, J. M. Dean, and A. J. Gunn. “Effect of Cerebral Hypothermia and Asphyxia on the Subventricular Zone and White Matter Tracts in Preterm Fetal Sheep." Brain Res 1469 (2012): 35-42.

83. Bennet, L., V. Roelfsema, S. George, J. M. Dean, B. S. Emerald, and A. J. Gunn. "The Effect of Cerebral Hypothermia on White and Grey Matter Injury Induced by Severe Hypoxia in Preterm Fetal Sheep." J Physiol 578, no. Pt 2 (2007): 491-506.

84. Wang, H., L. Zhang, and Y. Jin. "A Meta-Analysis of the Protective Effect of Recombinant Human Erythropoietin (Rhepo) for Neurodevelopment in Preterm Infants." Cell Biochem Biophys 71, no. 2 (2015): 795-802.

85. Park, W. S., S. I. Sung, S. Y. Ahn, H. S. Yoo, D. K. Sung, G. H. Im, S. J. Choi, and Y. S. Chang. "Hypothermia Augments Neuroprotective Activity of Mesenchymal Stem Cells for Neonatal Hypoxic-Ischemic Encephalopathy." PLoS One 10, no. 3 (2015): e0120893.

86. Daadi, M. M., A. S. Davis, A. Arac, Z. Li, A. L. Maag, R. Bhatnagar, K. Jiang, G. Sun, J. C. Wu, and G. K. Steinberg. "Human Neural Stem Cell Grafts Modify Microglial Response and Enhance Axonal Sprouting in Neonatal Hypoxic-Ischemic Brain Injury." Stroke 41, no. 3 (2010): 516-23. 
87. Ahn, S. Y., Y. S. Chang, S. I. Sung, and W. S. Park. “Mesenchymal Stem Cells for Severe Intraventricular Hemorrhage in Preterm Infants: Phase I DoseEscalation Clinical Trial." Stem Cells Transl Med 7, no. 12 (2018): 847-56.

88. Brines, M. L., P. Ghezzi, S. Keenan, D. Agnello, N. C. de Lanerolle, C. Cerami, L. M. Itri, and A. Cerami. "Erythropoietin Crosses the Blood-Brain Barrier to Protect against Experimental Brain Injury." Proc Natl Acad Sci U S A 97, no. 19 (2000): 10526-31.

89. Traudt, C. M., and S. E. Juul. "Erythropoietin as a Neuroprotectant for Neonatal Brain Injury: Animal Models." Methods Mol Biol 982 (2013): 113-26. 


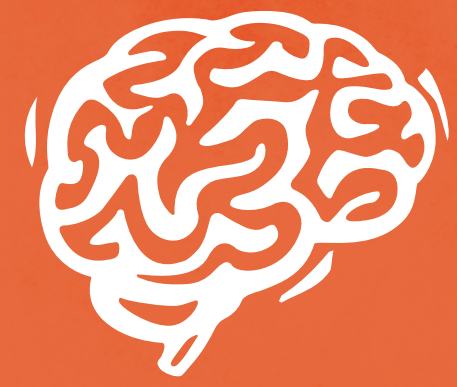




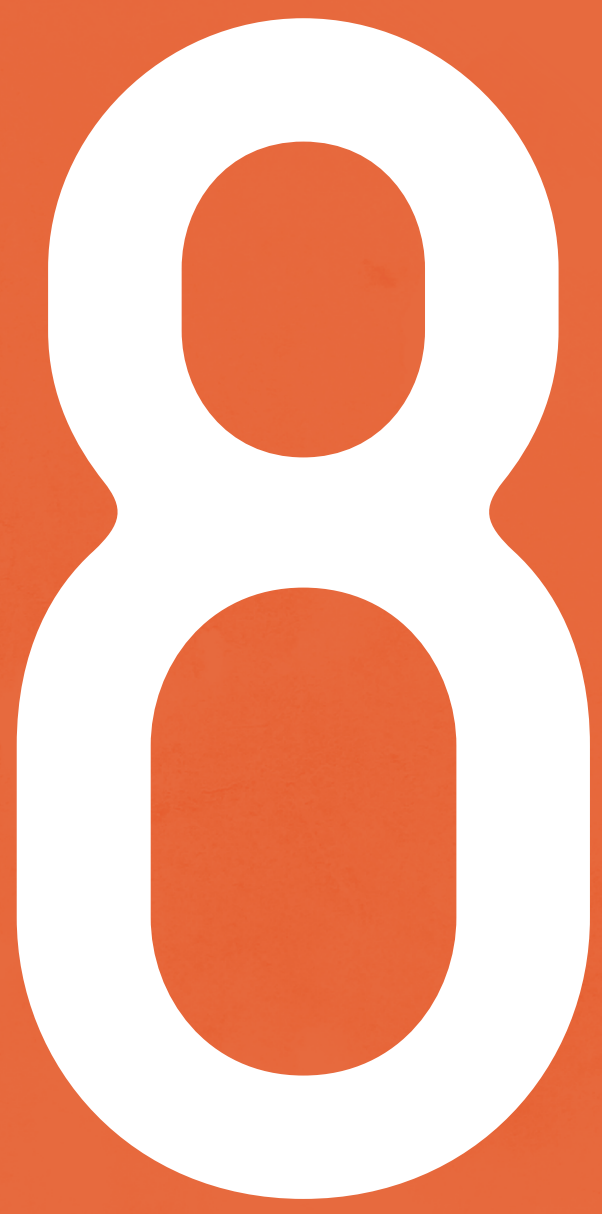

Nederlandse samenvatting 


\section{Nederlandse samenvatting}

Vroeggeboorte en inflammatoire stress rondom de geboorte zijn sterk geassocieerd met neurologische handicaps gedurende het gehele leven. In de afgelopen jaren is er uitgebreid onderzoek verricht om de onderliggende oorzaak en mechanismen die leiden tot deze hersenschade te identificeren om zo uiteindelijk nieuwe behandelingen te ontwikkelen. Echter, er blijven bepaalde essentiële vragen onbeantwoord waardoor deze resultaten maar in beperkte mate worden geïmplementeerd in de klinische praktijk. Het gevolg hiervan is dat tot op de dag van vandaag nog steeds veel vroeggeboren kinderen hersenschade ontwikkelen. In mijn thesis onderzoek ik het ontstaan van hersenschade na verschillende (gecombineerde) triggers, focus ik mij op de tijdsafhankelijke veranderingen in de hersenen van de vroeggeborene na deze triggers om zo het juiste tijdstip van behandelen beter te kunnen vaststellen en test ik nieuwe behandelingen.

EoP (hersenchade bij vroeggeborenen) heeft verschillende onderliggende oorzaken en ontstaansmechanismen. De twee meest belangrijke bijdragende factoren die ik onafhankelijk van elkaar heb bestudeerd in dit proefschrift zijn een intrauteriene ontsteking (chorioamnionitis) (hoofdstuk 2-4) en zuurstoftekort (globale hypoxie-ischemie) (hoofdstuk 5 \& 6). Om dit te bestuderen heb ik gebruik gemaakt van een preklinische groot proefdiermodel, het schaapmodel, welke de humane hersenontwikkeling tijdens de zwangerschap zeer nauwkeurig nabootst. In hoofdstuk 2-4 heb ik gebruik gemaakt van intra-uteriene infectie/inflammatie modellen welke de klinische acute/chronische chorioamnionitis met foetale betrokkenheid (FIRS) nabootst. In deze hoofdstukken worden de tijdsafhankelijke veranderingen en processen in het premature brein in kaart gebracht na blootstelling aan een intra-uteriene infectie (chorioamnionitis). Chorioamnionitis is een polymicrobieel ziektebeeld en in ons model maken wij gebruik van verschillende inflammatoire stimuli inclusief Ureaplasma Parvum (UP), C. albicans en E. coli-derived Lipopolysaccharide (LPS) welke de meest voorkomende bacteriën en schimmels in chorioamnionitis vertegenwoordigen. 
De eerste belangrijke bevinding van mijn werk is dat in de acute fase na blootstelling aan verschillende microbiële triggers (UP, Candida als LPS) een systemische immuunreactie optreedt die ik gekarakteriseerd heb door bepaling van het cytokine IL-6 in het foetale serum. Verhoogde systemische IL-6 levels is een essentiële marker voor het vaststellen van een foetaal inflammatory response syndrome (FIRS) welke in meerdere klinische studies is geassocieerd met neonatale mortaliteit en morbiditeit waaronder hersenschade inclusief cerebrale parese (CP). Onze data laat zien dat de IL-6 levels acuut en kortdurend verhoogd zijn na blootstelling aan intra-uteriene blootstelling aan inflammatie (UP, LPS) (hoofdstuk 2 \& 3). Om de bepaling van IL-6 levels als klinische biomarker voor het ontstaan van hersenschade te gaan gebruiken is het belangrijk om het tijdstip van deze bepaling mee te nemen in het beslismodel. Daarnaast zullen studies zich moeten gaan focussen op het combineren van bestaande systemische inflammatoire markers (CRP, procalcitonine) of nieuwe biomarkers die het ontstaan van een intra-uteriene infectie kunnen bepalen om zo de neurologische uitkomst beter te kunnen voorspellen in de toekomst.

In hoofdstuk 2 onderzoek ik de gevolgen van chronische intra-uteriene blootstelling aan Ureaplasma Parvum (UP) op het foetale brein. Ureaplasma species worden in vrouwen die te vroeg bevallen het meeste geïsoleerd uit het vruchtwater en placenta. Ondanks dat UP de meest voorkomende geïsoleerde microbe is bestaat er veel onenigheid over de rol van UP binnen de neonatale morbiditeit en mortaliteit. In mijn onderzoek laat ik zien dat chronische intra-uteriene blootstelling aan UP veranderingen in het foetale brein veroorzaken die op lange termijn zijn geassocieerd met een veranderde neurocognitieve ontwikkeling, waaronder verlies van astrocyten, epigenetische veranderingen en veranderingen in ontwikkelende witte stofcellen. Deze veranderingen kunnen verklarend zijn voor de geobserveerde veranderde neurologische uitkomsten op 2-jarige leeftijd in kinderen die geboren zijn en blootgesteld aan UP geïnduceerde chorioamnionitis.

Een volgende belangrijke bevinding laat zien dat in ieder infectieus/ inflammatoir model de systemische ontstekingsresponse (verhoogd IL-6) gevolgd wordt door een cerebrale response gekenmerkt door activatie van microglia, veranderingen in de witte stof maturatie en verhoogd aantal apoptotische cellen. Opmerkelijk is dat deze verhoogde celdood 
na blootstelling aan een inflammatoire trigger relatief later ontstaat in vergelijking met na een hypoxisch-ischemisch insult (hoofdstuk 3). Na blootstelling aan LPS zien wij een verhoogd aantal caspase-3 positieve cellen (marker voor apoptose) vanaf dag 8, waarbij dit na een globaal HI insult al vanaf dag 3 wordt waargenomen. Daarnaast wijzen de geobserveerde witte stof veranderingen op een maturatie arrest, een beeld dat naast het verlies aan witte stof als meest belangrijk onderliggend probleem gezien wordt in hersenschade van de vroeggeboren baby (EoP). Daarnaast maken deze veranderingen in de witte stof de baby vatbaar voor het ontwikkelen van neurologische handicaps gedurende het leven welke de $\mathrm{DOHaD}$ (developmental origin of health and disease) oftewel Barker hypothese ondersteunt. In mijn thesis worden verschillende oorzaken van deze veranderde witte stof maturatie als verklaring gevonden en onderbouwd waaronder afwijkende en overmatige microglia activatie, veranderingen in astrocyten populatie en epigenetische veranderingen. Echter een beperkende factor aan deze studies is de korte termijn follow-up. Lange termijn follow-up studies worden op dit moment uitgevoerd waarin de lange termijn gevolgen en de pathofysiologische processen onderliggend aan deze veranderingen bestudeerd zullen worden.

In hoofdstuk 4 heb ik de effecten van Candida albicans op het brein bestudeerd aangezien chorioamnionitis niet alleen wordt beperkt tot bacteriële triggers. Hoewel C. albicans geïnduceerde chorioamnionitis maar zelden voorkomt, zijn de effecten hiervan op de foetus enorm schadelijk. Ook in dit model zien wij een vergelijkbaar patroon aan veranderingen (verhoogd IL-6, cerebrale ontsteking en witte stof veranderingen). Een belangrijke bevinding vanuit deze studie is dat een eenmalige gift van intra-uteriene Fluconazole (antimycoticum) wel in staat is om neonatale sterfte te voorkomen maar niet de cerebrale ontstekingsresponse en potentieel schade op de lange termijn tegen gaat. Deze bevindingen pleiten ervoor dat een behandeling naast antimicrobiële/bacteriële eigenschappen ook immunomodulatoire/anti-inflammatoire effecten moet hebben.

\section{Behandelingen}

Therapeutische hypothermie is tot op heden de enige behandeling die hersenschade (deels) kan voorkomen die clinici voorhanden hebben voor pasgeboren baby's. Echter, $40 \%$ van de behandelde kinderen heeft ondanks hypothermie nog steeds afwijkende neurologische uitkomsten op de latere leeftijd. Daarnaast heeft hypothermie in vroeggeboren kinderen meer 
bijwerkingen en wordt afgeraden in deze kwetsbare groep kinderen. Meer recent onderzoek laat ook zien dat in de aanwezigheid van een infectie (chorioamnionitis) hypothermie helemaal niet effectief is en mogelijk zelfs nadelige gevolgen heeft. De noodzaak voor een nieuwe behandeling om EoP te voorkomen/behandelen is dus groot. Stamcellen zijn mogelijk een goede kandidaat hiervoor. Stamcellen bezitten zowel regeneratieve als immunomodulatoire eigenschappen. In eerder onderzoek heeft onze groep laten zien dat beenmerg afkomstige stamcellen (MSCs, MAPCs) in staat zijn om cerebrale ontsteking, witte stofschade en functieverlies als gevolg van zuurstoftekort kunnen voorkomen in het schapenmodel.

In hoofdstuk 5 laat ik zien dat deze MAPCs ook in staat zijn om het cerebellum (kleine hersenen) te beschermen tegen zuurstofgebrek rondom de geboorte. Schade aan het cerebellum speelt een toenemende belangrijke rol in de ontwikkeling van (niet-motorische) problemen na vroeggeboorte. $\mathrm{Na}$ zuurstoftekort rondom de geboorte (hypoxie-ischemie) ontstaat er een ontstekingsreactie, verlies van witte stof en verlies van belangrijke neuronen (purkinje cellen) in het cerebellum. Deze veranderingen passen bij een vertraagde of achterblijvende ontwikkeling van het cerebellum en worden geassocieerd met EoP. Ik laat zien dat MAPCs deze veranderingen kunnen voorkomen en hiermee dus problemen op latere leeftijd worden voorkomen.

In hoofdstuk 6 onderzoek ik het onderliggende mechanisme van deze stamceltherapie. De mechanismen waarop stamcelen het immuunsysteem kunnen beïnvloeden zijn namelijk grotendeels onbekend. Een voorgesteld mechanisme is door uitscheiding van extra-cellulaire blaasjes (EVs) waarin immuun modulerende moleculen verpakt zitten. In ons schaapmodel hebben wij eerder laten zien dat EVs afkomstig van stamcellen (MSC-EVs) in staat zijn om de duur en aantal convulsies te verminderen na zuurstoftekort. Daarnaast was er een neiging tot witte stof bescherming. Echter, in tegenstelling tot de stamcel zelf kon dit beschermend effect van de EVs niet verklaard worden door anti-inflammatoire effecten. Als alternatief werkingsmechanisme laat ik zien in deze studie dat MSC-EVs in staat zijn de bloed-breinbarrière (BBB) te herstellen na een periode van zuurstoftekort rondom de geboorte. Het is algemeen bekend dat de BBB na zuurstoftekort beschadigd raakt waardoor schadelijke immuuncellen uit het bloed de hersenen kunnen binnendringen en hier bijdragen aan de ontstekingsresponse en witte stofschade. Daarnaast laten wij zien dat deze 
verstoring in barrière gepaard gaat met een vermindering van AnnexineA1 (ANXA1) expressie in het brein. ANXA1 staat bekend als een regulator van de bloed-hersenbarrière en speelt ook een rol in het ontstaan van een beschadigde barrière in ziekten als Parkinson en Multipele Sclerose. In een kweekmodel toon ik aan dat door het toedienen van zowel MSC-EVs als ANXA1 de bloed-hersenbarrière wordt hersteld na een periode van zuurstoftekort. Deze bescherming van de BBB komt door signalering van ANXA1, via de ANXA1/FPR-axis welke het tight junction complex van de endotheelcellen versterkt. Concluderend, het beschermende effect van MSC-EVs kan (deels) worden verklaard door het beschermende effect van de bloed-hersenbarrière, welke wordt gemedieerd door ANXA1.

Tot slot heb ik in hoofdstuk 3 ingezoomd op de regulatie van het receptor systeem van een ander potentieel neuro-farmacologische toepassing namelijk het al bekende cytokine Erytropoëtine (EPO). Zowel uit deze studie, als ook uit de data van het ANXA1/FPR receptor systeem (hoofdstuk 6), blijkt dat er op specifieke tijdstippen na een pro-inflammatoir insult in utero, er veranderingen ontstaan in deze complexen welke inzicht verschaffen over mogelijke timing van start van behandelingen. Zo ontstaat er na blootstelling aan een pro-inflammatoir insult over de tijd een depletie ontstaat van het endogene ANXA1. Tevens hebben we een verminderde activiteit van de EPO-receptor aangetoond. Deze resultaten vormen een mogelijke verklaring voor de wisselende uitkomsten van klinische studies waarin EPO als mogelijk behandeling in pasgeborenen werd getest. Tevens hebben we voor het potentieel klinisch gebruik van AnxA1 een "optimal window of opportunity" blootgelegd.

\section{De toekomst}

Hersenschade bij vroeggeborenen is multifactorieel en complex en kent een zeer heterogene patiëntenpopulatie. Ieder kind heeft een eigen "vingerafdruk" van risicofactoren die een rol spelen in het ontstaan en verloop van mogelijke hersenschade. Dit behoeft een individuele aanpak wat betreft de behandeling (personalised medicine). In mijn onderzoek breng ik tijdsafhankelijke veranderingen in het premature brein in kaart na inflammatoire blootstelling in utero. Belangrijk hierin is dat ik laat zien dat er mogelijk een mismatch bestaat tussen het optimale moment waarop behandeling het meest effect zou zijn en het tijdstip waarop er in de kliniek daadwerkelijk behandeling wordt gegeven. Om een individuele aanpak 
te implementeren in de kliniek is stratificatie middels betrouwbare biomarkers een vereiste. De timing en het type insult spelen hierbij een sleutelrol. Afhankelijk van de voorgeschiedenis van de pasgborene kan een op maat beleid worden afgesteld. Interventies middels stamcellen, Annexine A1 en EPO (of een combinatie hiervan) zijn in dit kader veelbelovend. 


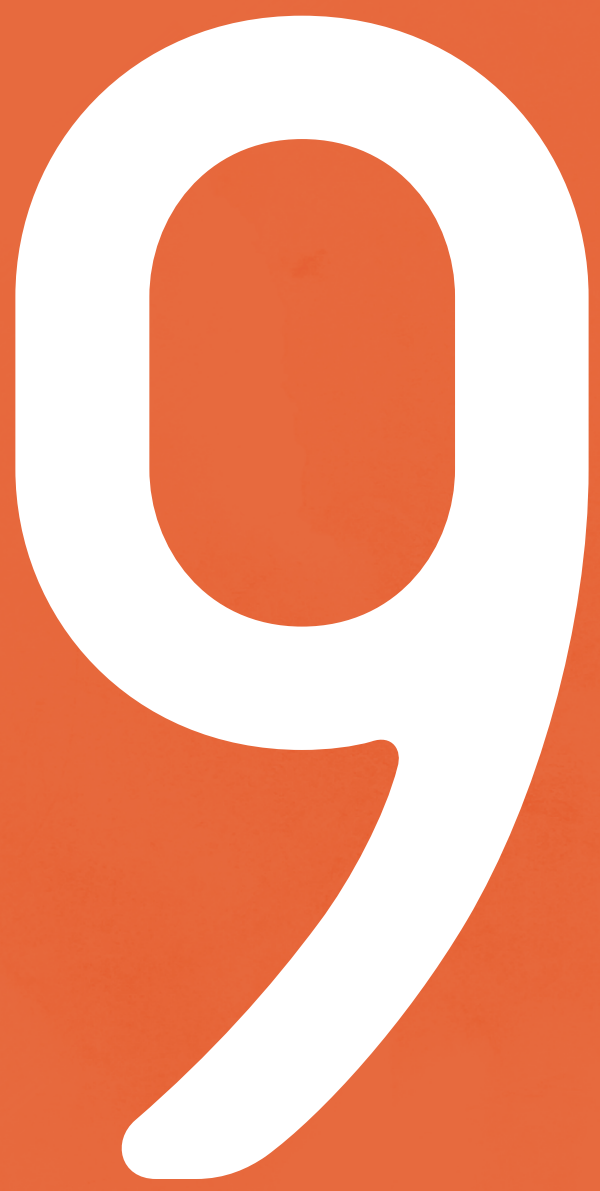

Valorization 



\section{Valorization}

Over the past decades, improved perinatal health care has led to more infants that survive preterm birth and consequently a shift in survival to earlier gestational ages [1]. However, the chance of event-free survival is still low in extremely preterm babies. Nowadays, around $35 \%$ of preterm infants survive with neurodevelopmental impairments such as cognitive deficits and attention deficit disorder which rises up to 50-60\% in extremely preterm babies ( $<27$ weeks of gestation), and around 10\% develop motoric spasticity (CP) [2]. This results in a prevalence of $\mathrm{CP}$ around two per 1000 live births in developed and in developing countries [3, 4]. Assuming that in the Netherlands 180.000 children are born annually, this accounts for 360 babies born with CP every year. EoP does not only affect the patient but has an enormous impact on its families and society $[5,6]$. Substantially increased lifetime costs are therefore attributed to EoP. Annually, in the Netherlands the costs for all CP patients comprises $€ 737,5$ million Euros (0,10\% of BNP) [4].

In this thesis, I addressed the time dependent changes in the preterm brain in the course of perinatal stress and identified (cell-based, cell-derived and anti-infective) therapies. For this purpose, we used a translational ovine model of preterm brain injury caused by either HI or intrauterine infection. The long gestational period of sheep enables us to study antenatal developmental processes in detail and to administer our therapies at specific time-points in during this development. Moreover, ovine brain development in utero and the cerebral disease progression following perinatal inflammatory insults (global HI and chorioamnionitis) has essential overlap with man [7].

The data presented in this thesis (chapter 2-6) support and extend the concept that EoP has a multifactorial and complex origin, indicating that every newborn child has its own "fingerprint" of risk factors potentially contributing to EoP development. More precisely, the timing of the peripheral and cerebral inflammation and its pathophysiological cerebral 
changes were shown to be different following a sterile (HI) insult when compared to an infectious trigger (chapter $3 \&$ ). Accordingly, we show that defined windows of opportunity emerge following exposure to multiple perinatal inflammatory triggers. These important findings highlight that a potential mismatch might be present between the optimal timing of a treatment (in relation to the nature of the insult(s)) and the current clinical initiation of a treatment. This mismatch impedes development and translation of neurological therapeutics for preterm infants. Correct timing of treatment initiation in relation to the distinct nature and stage of injury is of great clinical importance in the near future. Translation of our findings into the clinical setting demands diagnostic tools for the recognition of these perinatal risk factors in a safe and non-invasive manner. Our group is currently working on analyzing volatile organic compounds in breath condensate of pregnant women to determine the presence and/or predict the onset of intra-amniotic infections.

In addition, we performed diffusion MRI sequencing in our study to further improve the clinical use of DTI as a biomarker of individual neurodevelopment and therapeutic effect in the future. Correlation of such new crucial imaging biomarkers with microstructural histological changes will improve clinical application and subsequently result in an individualized treatment approach (chapter 5). Such personalized medicine in which tailor made treatments will be adjusted to individuals need is nowadays focus of research in every facet of health care, from prevention to terminal care. The overall aim is to develop new methodologies and technological applications that make it easier to predict outcomes in individual cases, align treatment with those predictions, and administer reliable tests to determine the success of that treatment. Closer cooperation and back-to-back analysis between translational/basic science and clinical data will help to achieve this aim.

Our data (chapter 4) suggests that eradication of Candida Albicans with anti-fungal treatment is not sufficient to prevent brain injury. Our work in chapter 3 supports other reports that describe that cerebral inflammation that is initiated upon exposure with an infectious trigger continuous (postnatally) and might even persist into adulthood. Therefore, future treatment protocols should combine multiple therapeutics that harbor anti-microbial, immunomodulatory and regenerative capacities to protect 
against neurodevelopmental disorders in later life. Potential candidates that have proven to possess anti-inflammatory or immunomodulatory capacities are erythropoietin, cell-based therapies including MSCs/MAPCs (chapter 3 \& 5) and AnnexinA1 (chapter 6). In chapter 6, we investigate the potential therapeutic role of AnxA1 in hypoxic-ischemic encephalopathy (HIE). We are the first group to demonstrate a strong potential for AnxA1 in the treatment of HI-induced brain injury. The intellectual property has been patented which offers new scientific and economic opportunities. Future research should focus on route, dose and timing of AnxA1 and elucidate whether AnxA1 can act synergistically with other therapies including cell-based therapies like MAPCs (Multistem $\left.{ }^{\circledR}\right)$ that have been successfully tested (chapter 5). The potential for MultiStem ${ }^{\circledR}$ to treat ischemic stroke is currently being evaluated in a registered trial in Japan and in a pivotal Phase 3 clinical trial conducted in North America and Europe (clinicaltrial.gov). In addition, this program received the Fast Track designation, as well as the Regenerative Medicine Advanced therapy designation from the FDA (fda.gov). These designations are designed to accelerate the development, regulatory review and subsequent commercialization of product candidates like MultiStem ${ }^{\circledR}$ cell therapy for ischemic stroke if the clinical evaluation demonstrates appropriate safety and therapeutic effectiveness. As a subsequent step, MAPCs can be evaluated as therapeutic intervention in neonatal brain injury. In conclusion, the findings in this thesis are on short term primary of value to other researchers, but in long term could be translated into commercially available products and/or services to improve the outcome of babies at risk for EoP. 


\section{References}

1. “Perinatale Zorg in Nederland 2017. ." Utrecht: Perined, 2019.

2. Volpe, J. J. "The Encephalopathy of Prematurity--Brain Injury and Impaired Brain Development Inextricably Intertwined." Semin Pediatr Neurol 16, no. 4 (2009): 167-78.

3. Blair, E. "Epidemiology of the Cerebral Palsies." Orthop Clin North Am 41, no. 4 (2010): 441-55.

4. Sellier, E., M. J. Platt, G. L. Andersen, I. Krageloh-Mann, J. De La Cruz, C. Cans, and Network Surveillance of Cerebral Palsy. "Decreasing Prevalence in Cerebral Palsy: A Multi-Site European Population-Based Study, 1980 to 2003." Dev Med Child Neurol 58, no. 1 (2016): 85-92.

5. Blencowe, H., S. Cousens, M. Z. Oestergaard, D. Chou, A. B. Moller, R. Narwal, A. Adler, C. Vera Garcia, S. Rohde, L. Say, and J. E. Lawn. "National, Regional, and Worldwide Estimates of Preterm Birth Rates in the Year 2010 with Time Trends since 1990 for Selected Countries: A Systematic Analysis and Implications." Lancet 379, no. 9832 (2012): 2162-72.

6. Lawn, J. E., R. Davidge, V. K. Paul, S. von Xylander, J. de Graft Johnson, A. Costello, M. V. Kinney, J. Segre, and L. Molyneux. "Born Too Soon: Care for the Preterm Baby." Reprod Health 10 Suppl 1 (2013): S5.

7. Back, S. A., A. Riddle, and A. R. Hohimer. "Role of Instrumented Fetal Sheep Preparations in Defining the Pathogenesis of Human Periventricular White-Matter Injury." J Child Neurol 21, no. 7 (2006): 582-9. 


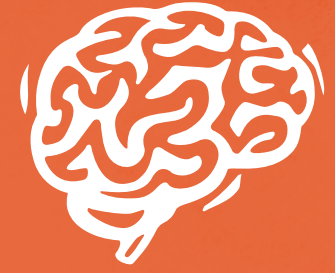



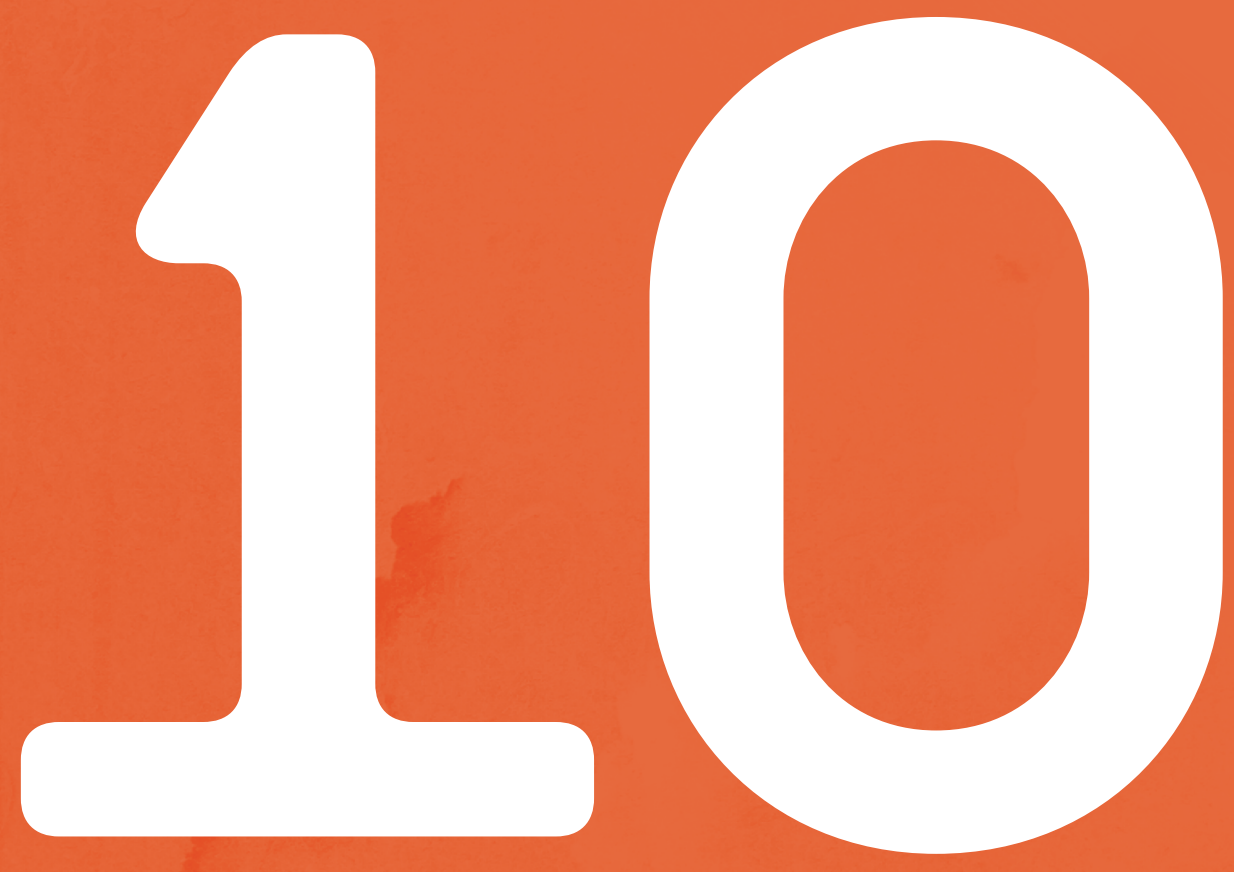

$$
\begin{array}{r}
\text { Curriculum Vitae } \\
\& \text { List of } \\
\text { publications }
\end{array}
$$




\section{Curriculum Vitae}

Ruth Gussenhoven werd geboren op 2 januari 1989 in Echt, Limburg. Na het behalen van het VWO diploma aan het Bisschoppelijk College te Echt begon zij in 2007 haar Bachelor Geneeskunde aan de Universiteit van Utrecht en vervolgde zij haar opleiding met de Master in Geneeskunde aan de Universiteit van Maastricht. Vanwege de interesse in de embryologie en ontwikkelings(patho)fysiologie en ambities binnen de perinatologie koos zij voor een keuzestage op de Neonatale Intensive Care Unit in het MUMC+. Haar Master sloot zij af met een stage binnen de kinderradiologie onder supervisie van prof. Dr. Simon Robben en een wetenschapsstage in het onderzoekslaboratorium van prof. Boris Kramer en dr. Tim Wolfs. Hier deed zij onderzoek naar de effecten van intra-uteriene blootstelling aan infecties op de ontwikkeling van de foetale hersenen. Gedurende deze stages groeide haar interesse in de ontwikkelings(patho)fysiologie van de hersenen. In het bijzonder de sterke ontwikkelingen binnen dit vakgebied en het gebrek aan goede behandelopties voor deze kinderen dreven haar om dit onderzoek voort te zetten. Na het succesvol behalen van haar artsendiploma in 2014 startte zijn haar promotietraject waar zij van 2014-2018 aan heeft gewerkt. De resultaten van dit onderzoek staan in dit proefschrift beschreven. Vanaf mei 2018 is zij werkzaam als arts-assistent (niet in opleiding) Kindergeneeskunde in het Maxima Medisch Centrum in Veldhoven.

Ruth Gussenhoven was born on 2th of January 1989 in Echt (Limburg, the Netherlands). After finishing secondary school at Bisschoppelijk College in Echt she started her Bachelor in Medicine at the University of Utrecht in 2007 followed by her Master in Medicine at the University of Maastricht. Driven by her interest in embryology and developmental (patho)physiology she choose for an elective internship at the Neonatology Intensive Care Unit at MUMC+. At the end of her Master studies she performed an internship at the Pediatric Radiology department under supervision of Prof. Simon Robben and a scientific internship at the research laboratory under supervision of prof. dr. Boris Kramer and dr. Tim Wolfs. During the scientific internship she studied the effects of intra-uterine exposure of infections on the development of the fetal brain. In 
particular, the development within this discipline and the lack of an effective treatment for these vulnerable children encouraged her to continue this research. After successfully obtaining her doctor's degree in 2014, she started her PhD training which resulted in this thesis. From May 2018 she works as a resident (not in training) in Pediatrics at the Maxima Medical Center in Veldhoven. 


\section{List of publications}

- Systemic Multipotent Adult Progenitor Cells protect the cerebellum after asphyxia in preterm fetal sheep.

Ruth Gussenhoven, Jeroen Dudink, Kay Pieterman, Martin Lammens, Daan R.M.G. Ophelders, Robert W. Mays, Luc J. Zimmermann, Boris W. Kramer, Tim G.A.M. Wolfs, Reint K. Jellema.

Resubmitted in Stem Cells translational medicine

- Annexin A1 as Neuroprotective Determinant for Blood-Brain Barrier Integrity in Neonatal Hypoxic-Ischemic Encephalopathy.

Gussenhoven R, Klein L, Ophelders DRMG, Habets DHJ, Giebel B, Kramer BW, Schurgers LJ, Reutelingsperger CPM, Wolfs TGAM.

J Clin Med. 2019 Jan 24;8(2). pii: E137.

- The influence of anesthetics on substantia nigra tyrosine hydroxylase expression and tau phosphorylation in the hypoxic-ischemic near-term lamb.

Barkhuizen M, van Dijck FJP, Jellema RK, Gussenhoven R, Engelbertink I, van Mechelen R, Cleutjens JPM, Seehase M, Steinbusch HWM, Zimmermann LJ, Gavilanes AWD, Kramer BW.

Pediatr Res. 2018 Jun;83(6):1190-1199.

- Chorioamnionitis, neuroinflammation, and injury: timing is key in the preterm ovine fetus.

Gussenhoven R, Westerlaken RJJ, Ophelders DRMG, Jobe AH, Kemp MW, Kallapur SG, Zimmermann LJ, Sangild PT, Pankratova S, Gressens P, Kramer BW, Fleiss B, Wolfs TGAM.

J Neuroinflammation. 2018 Apr 19;15(1):113. . 
- The Paradoxical Effects of Chronic Intra-Amniotic Ureaplasma paroum Exposure on Ovine Fetal Brain Development.

Gussenhoven R, Ophelders DRMG, Kemp MW, Payne MS, Spiller OB, Beeton ML, Stock SJ, Cillero-Pastor B, Barré FPY, Heeren RMA, Kessels L, Stevens B, Rutten BP, Kallapur SG, Jobe AH, Kramer BW, Wolfs TGAM. Dev Neurosci. 2017; 39(6):472-486.

- Neuroinflammation and structural injury of the fetal ovine brain following intraamniotic Candida albicans exposure.

Gussenhoven $\mathbf{R}^{*}$, Ophelders DR*, Lammens M, Küsters B, Kemp MW, Newnham JP, Payne MS, Kallapur SG, Jobe AH, Zimmermann LJ, Kramer BW, Wolfs TG.

J Neuroinflammation. 2016 Feb 2;13:29. doi: 10.1186/s12974-016-0492-z.

- Intra-Amniotic LPS Induced Region-Specific Changes in Presynaptic Bouton Densities in the Ovine Fetal Brain.

Strackx E, Jellema RK, Rieke R, Gussenhoven R, Vles JS, Kramer BW, Gavilanes AW.

Biomed Res Int. 2015; 2015:276029.

- Pulmonary Artery Aneurysm as a Clue to Behcet's Disease in a 7-Year-Old Boy. Ruth Gussenhoven, Simon Robben, Jannie Hof, Francois Vermeulen, Gijs van Well

Int J Clin Pediatr. 2015 


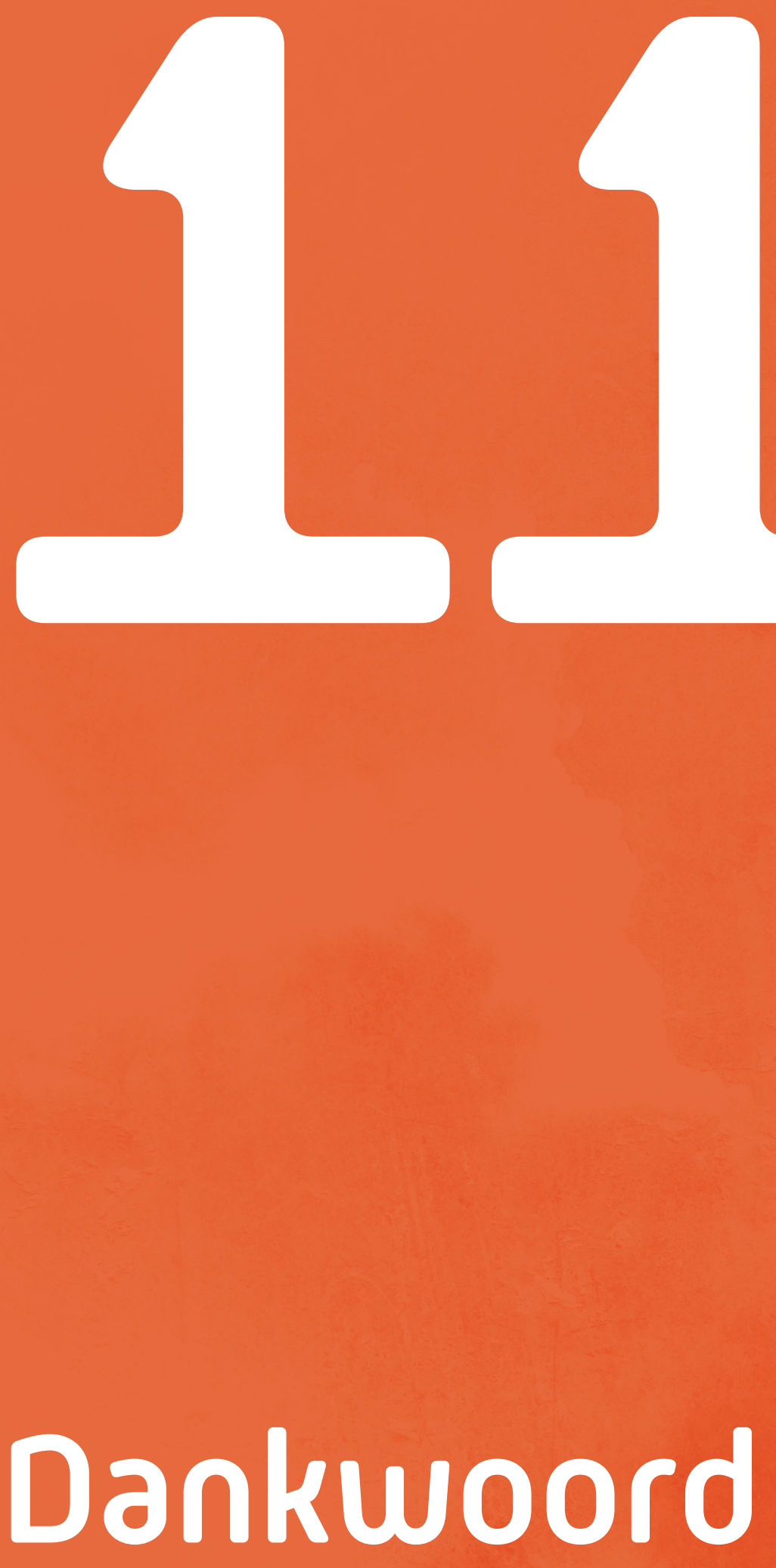





\section{De finish...}

Met een goede fles wijn begin ik aan dit laatste hoofdstuk uit mijn boekje. Met een lach kijk ik terug op een bijzondere tijd uit mijn leven. Maar ook bloed, zweet en tranen zijn mij niet bespaard gebleven. $\mathrm{Nu}$ zijn de laatste paar meters aangebroken, de finish is in zicht, en is eronderuit komen echt geen optie meer: het gaat gebeuren! In dit laatste hoofdstuk mag ik alle fijne mensen bedanken die mij de afgelopen jaren zo ontzettend goed geholpen hebben en zonder wie dit resultaat nooit zo mooi was geworden. En ook de mensen die mij iedere keer opnieuw hebben gemotiveerd en op de moeilijke momenten hebben gestimuleerd of afgeleid ben ik ontzettend dankbaar.

Te beginnen met mijn promotieteam bestaande uit dr. Tim Wolfs, prof. Dr. Boris Kramer en prof. Dr. Luc Zimmermann, zonder wie ik niet de kans had gekregen om mijn onderzoeksstage voort te zetten in dit PhD traject. Als jonge bijna afgestudeerde dokter heb ik hier mijn eerste stappen in de onderzoekswereld mogen zetten en mij uiteindelijk mogen ontwikkelen tot een volwaardig onderzoeker.

Tim, ik weet zeker dat zonder jouw gedrevenheid en begeleiding ik nooit zo ver was gekomen. Je optimisme, kritische houding en brede kennis en ervaring in het onderzoek gaven mij de sturing die ik nodig had. Telkens wist jij mij te stimuleren om het beste uit mezelf te halen en mijn doel niet uit het oog te verliezen. Messcherp moest het zijn en alles werd tot in de puntjes geperfectioneerd, want goed was niet goed genoeg. Uiteindelijk resulterend in een aantal echt mooie publicaties waar we trots op mogen zijn. Gelukkig werd dit harde werken ook afgewisseld met heel hard lachen (anekdotes om een heel boek mee te schrijven dus ik zal het je hier besparen). Dit maakte het harde werken nooit vervelend en ook de moeilijke momenten makkelijker te relativeren. Ik heb ontzettend veel van je geleerd en heb bewondering voor hoe jij leidinggeeft over de groep. Bedankt voor de steun die je mij hebt gegeven en ik ben er trots op dat ik onder jouw supervisie mag promoveren. 
Boris, "Als jij neonatoloog wil worden, moet je onderzoek komen doen bij mij." was het eerste wat je tegen mij zei bij onze ontmoeting. Je enthousiasme voor het vakgebied werkte aanstekelijk en als net afgestudeerde jonge dokter werd ik geboeid op de manier hoe jij sprak over deze groep kwetsbare patiëntjes van de NICU. Mijn onderzoekstijd werd dan ook regelmatig afgewisseld met een bezoek aan de NICU. Bedankt voor het mede mogelijk maken voor de kans om mij als onderzoeker te ontwikkelen en ook om hierbij het klinische aspect niet uit het oog te verliezen.

Luc, bedankt voor de kritische input met betrekking tot de inhoud van mijn onderzoek maar vooral ook bedankt voor het zijn van een luisterend oor op de momenten waar ik die nodig had. Heel veel succes en plezier met je nieuwe baan.

De leden van de leescommissie, prof. Dr. Jeroen Vermeulen, prof. Dr. Marc Spaanderman, dr. Daniel van den Hoven, Prof. Dr. Freek Hoebeek en dr. Jane Pillow, wil ik bedanken voor het beoordelen van mijn proefschrift. Het positieve oordeel van deze groep maken voor mij dit proefschrift extra bijzonder! Beste Jeroen, bovendien wil ik je bedanken voor je toegankelijkheid en behulpzaamheid over de afgelopen jaren zowel vakinhoudelijk maar ook als ik vastliep met MRI-software. Ik mocht altijd bij je aankloppen. Ook de sessies waarin we brainstormden om nieuwe MRI-projecten van de grond te krijgen zijn uiteindelijk niet voor niets geweest. Ik kijk enorm uit naar alle resultaten van de aankomende studies. Beste Freek, we zijn ooit in Rotterdam begonnen met onze samenwerking en inmiddels zijn we je gevolgd naar Utrecht. Je scherpe inbreng en vermogen om iets vanuit andere invalshoeken te bekijken samen met je fundamentele neurowetenschappelijke kennis waren vaak van toegevoegde waarde waren voor mijn studies. Daarnaast zijn de gezamenlijke meetings tussen onze onderzoeksgroepen, ook samen met Jeroen, Kay, Cora en Caroline, waarin we brainstormen over onze studies een ontzettend leuk en inspirerend aspect van onderzoek. De biertjes na afloop maken het natuurlijk nog gezelliger en een treinritje naar Utrecht geen straf. Ik hoop dat de samenwerking tussen beide groepen voor de toekomst nog vele mooie projecten gaan opleveren. Dear Jane, thank you for the critical appraisal of my thesis and granting it with a positive result. It was nice meeting you at the JENS conference in Maastricht this year. 
Ontzettend blij ben ik met mijn twee paranimfen, Daan en Lilian, die deze dag naast mij staan! Maar veel belangrijker is dat jullie de afgelopen jaren mijn 'lab' maatjes zijn geweest. Ik heb veel steun aan jullie gehad. Zonder jullie was mij dit nooit gelukt. Daan, ooit ben ik begonnen als jouw student en was jij voor mij als begeleider iemand met een enorme brede kennis en passie voor dit onderzoek. Daarnaast deelden we de interesse in de ontwikkelings(patho)fysiologie van de hersenen waarover we uren konden filosoferen en brainstormen over nieuwe experimenten. Hierdoor voelde ik me al snel thuis in het laboratorium. Als onderzoeker ben jij een onuitputtelijke bron van nieuwe ideeën en hadden we helaas vaak tijd te kort om deze allemaal uit te voeren. Tijdens onze zoektochten naar steeds betere koffiebarretjes in en rond het ziekenhuis hebben we ook ontzettend veel gelachen, praatte jij mij weer bij over de 'achterklap'-roddels maar kon ik ook mijn frustraties en moeilijkheden bij je kwijt. Daan, je bent een ontzettend fijn mens en goede collega, bedankt dat je er voor mij bent geweest en ik heb niemand liever naast mij staan op deze dag dan jij! Lilian, jij verdient ook een ontzettend grote pluim voor al het werk dat jij hebt verzet in het lab. Jij was mijn extra paar gouden handen! Zonder al te veel moeite wist jij ieder protocol werkende te krijgen en konden we vaak samen enorme reeksen aan experimenten verzetten. Daarnaast hebben we veel gelachen en gek gedaan (met het foute uur op de achtergrond), zette je me iedere ochtend een bakje verse koffie en kon ik ook als ik ergens tegenaan liep goed met je praten. Je heerlijke nuchtere kijk op dingen, die ik weleens mis (en niet alleen op de lab-uitjes;)) waren vaak wel helpend. Ik ben blij met jou naast mij op deze dag!

Mijn andere (ex)collega's van het kindergeneeskunde lab. Maria, Monique, Sizzle, Jip, Reint \& Matthias. Thanks for welcoming me as your newest roommate and introducing me in the world of sheep science. Maria, I hope the Greek sun is treating you well and that you never feel cold anymore! I miss you as our roomie, but we can finally open the windows for some fresh air. Jip, bedankt voor de gezelligheid en de flauwe 'plastische' humor die ik stiekem wel kon waarderen. Ik wens je ontzettend veel succes met de laatste loodjes en het krijgen van een opleidingsplek, je hebt het verdiend! Reint, het zien van jouw verdediging was voor mij de eerste keer dat ik deze ceremonie zag waarbij ik al plaatsvervangende zenuwen kreeg, erg indrukwekkend. Ik heb alleen maar respect voor hoe jij de kliniek combineert met de wetenschap en hard door bikkelt aan je carrière. Een bijna onmogelijke combinatie lijkt mij, heel knap! Mijn laatste project 
ontstond vanuit onze nieuwsgierigheid naar de kleine hersenen welke we uiteindelijk samen hebben opgepakt. De weg was af en toe hobbelig, de laatste loodjes zijn het zwaarst, maar ik denk dat we uiteindelijk tevreden mogen zijn met het resultaat. Bedankt voor je begeleiding en vooral ook het geduld, zeker voor het afgelopen jaar. Beste Matthias, bedankt voor de gezelligheid tijdens de koffie breaks en ook voor je luisterend oor en adviezen die je me hebt gegeven afgelopen jaren. Wat zou het leuk zijn om in de toekomst ook eens samen dienst in de kliniek te mogen draaien.

Het lab zou nooit overeind blijven zonder de ondersteunende rol van Nico, Leon en Teun, welke van onschatbare waarde is geweest. Samen zorgen jullie voor orde in de chaos maar ook voor een gezellige ontspannen sfeer waarbij er naast hard werken ook veel gelachen kon worden. Bedankt! Teun, je optimisme en warmte worden nog iedere dag gemist op het lab! De lab-uitjes met jou blijven voor altijd een mooie herinnering!

Mijn nieuwe collega's, Luise, Helene, Ilse \& Charlie, ik mis jullie als mijn teamgenootjes! Ik heb veel bewondering voor hoe jullie samen dit gigantische project toch maar even weten neer te zetten, echt teamwork! Luise, ik ben dankbaar voor je hulp in het afronden en opschrijven van het Annexine project. Super goed gedaan! Ik ben heel benieuwd wat de toekomst van Annexine ons gaat brengen. Charlie, bedankt voor onze nachtdienst samen met het eerste lammetje. Dat was wel heel bijzonder! Bedankt voor je Brabantse gezelligheid en je lieve woorden. Jij bent toch echt de volgende in de rij en ik weet zeker dat je dat fantastisch zult gaan doen!

Een groot aantal aan studenten hebben mijn onderzoek ondersteund in de afgelopen jaren. Ik wil jullie graag bedanken voor jullie inzet, enthousiasme en motivatie. Met name Ilse, Bas, Denise, Rob, Pieter, Michelle, Elise, Tamara, Dianne \& Miro. In het bijzonder, Denise, als lid van team Xtreme is het jou toch maar even mooi gelukt om het Annexine project van de grond te krijgen. Je mentaliteit, positieve instelling en ambitie zijn een groot aandeel geweest in het slagen van dit project, je bent een topper! Dat je je voor je eigen $\mathrm{PhD}$ opnieuw in een onbekend en ingewikkeld onderwerp hebt gestort vind ik alleen maar erg knap. Jij gaat geen uitdaging uit de weg! Ook ben ik fan van de sportieve, reislustige uitdagingen die jij zoekt naast je werk. Mocht je nog eens een te gek idee hebben, laat het me dan vooral weten. 
Rob, bedankt voor je hulp in het LPS-kinetics project. Jouw onuitputtelijke energie, positiviteit en gedrevenheid zijn bewonderenswaardig en jaloersmakend. Het lijkt wel alsof jouw dag meer dan 24 uur bevat. Knap hoe jij een opleiding tot kinderarts weet te combineren met het opzetten van een nieuw project voor je eigen onderzoek. Ik kijk heel erg uit naar je eerste resultaten, ontzettend veel succes!

Alle coauteurs wil ik bedanken voor hun gewaardeerde input en feedback die ik heb mogen ontvangen voor mijn studies. Chris Reutelingsperger en Leon Schurgers van de afdeling Biochemie wil ik graag bedanken voor de ontzettend fijne samenwerking en het samenbrengen van Annexines en de kindergeneeskunde. Ik ben ontzettend enthousiast over de eerste resultaten en ben heel benieuwd wat de samenwerking in de toekomst gaat brengen. Chris, ook wil ik je bedanken voor het deelnemen in mijn corona. Jeroen Dudink en Kay Pietermans wil ik speciaal bedanken voor de hulp in het analyseerbaar maken van de MRI-data, jullie inhoudelijke bijdrage voor de papers en de gezelligheid tijdens onze ontmoetingen. Jeroen nog bedankt voor je gastvrijheid en het lenen van je kamer in het WKZ om mij zo een weekje af te zonderen en de analyses te verrichten. Kay heel veel succes met je verdere opleiding tot radioloog. Bart Rutten, bedankt voor je input met betrekking tot de epigenetica. Heel veel dank aan de pathologen, Benno Kusters en Martin Lammens, voor jullie expertise in het beoordelen van onze studies. Jullie bijdrage is zeer waardevol geweest voor de publicaties. Bobbi Fleiss \& Pierre Gressens, thank you very much for the contributions to my work. You are a great example for me as a researcher and I admire your work.

Daarnaast zijn er nog collega's van verschillende andere afdelingen geweest die ieder op zijn manier enorm geholpen hebben om dit boekje tot stand te brengen. Hieronder vallen onder andere Jack Cleutjens en Benoit Frere van de afdeling Pathologie. Jack, mijn idee"en voor histologische analyses waren voor jou nooit te vreemd, en het lukte je dan ook altijd om hier een 'quip' voor te ontwerpen. Dit heeft mijn PhD-leven een stuk makkelijker gemaakt. Ontzettend bedankt. Benoit, als unitleider van het histologie-lab van het ziekenhuis wisten weje altijd te vinden voor een protocol of een snelle reeks aan automatische kleuringen. Ook dit maakte ons leven makkelijker. Heel veel dank hiervoor. Van de Mental Health and Neuroscience groep wil ik Harry Steinbusch bedanken voor de kans om mijn onderzoek te mogen doen onder de paraplu van MHeNs en Hellen Steinbusch voor de 
technische ondersteuning op het lab. Ook Nico en Lisette van

Biochemie bedank ik voor de technische ondersteuning en hulp met betrekking tot de experimenten. From the M4I department I would like to thank Berta and Karolina for explaining me repeatedly and patiently the basics of mass spectroscopy. Without you, I would have felt lost in translation with respect to the mass spectroscopy.

Naast het werken was er ook tijd voor ontspanning, ben ik nieuwe sportieve uitdagingen aangegaan en heb ik dit gecombineerd met verschillende bezoekjes aan Afrika en het steunen van de Flying Doctors. Een aantal mensen in het bijzonder zijn dit met mij aangegaan en hebben dit mede mogelijk gemaakt. Team the Big-6, Eva, Linda, Arjen, Kyra en Bart, ontzettend bedankt voor zowel het plezier in de voorbereidingen als ook de tijd in Tanzania. Het fietsen van de Africa Classic is voor mij een onvergetelijk mooie ervaring geweest en ik vond het heel gezellig dat ik dit samen met jullie mocht meemaken. Adhiambo Witlox en Stijn Bolink van de Orthopedie, bedankt voor de ontzettend leuke en leerzame tijd die ik met jullie mocht doorbrengen in Ghana in het St. John of God hospital. Dit stukje kliniek gaf mij midden in mijn promotieonderzoek weer de juiste drive om door te gaan. Hier werd ik weer herinnerd aan hoe mooi het werk is dat we als dokter mogen doen. Iets wat je toch met momenten uit het oog verliest wanneer je ondergedompeld zit in het laboratoriumwerk. Ontzettend mooi dat jullie deze samenwerking vanuit Maastricht blijven faciliteren. Daarnaast wil ik Adhiambo ook bedanken voor het te gekke idee wat in Africa is ontstaan om samen met Mark deel te nemen aan de IronMan Maastricht! Mijn oude zwem carrière heb ik weer een boost gegeven en al deze zwemtrainingen hebben mij echt de nodige ontspanning gegeven tijdens dit traject. Als toppunt heb ik 3,8 kilometer door de Maas afgelegd en uiteindelijk samen met jullie over de finish gerend, wat een adrenaline! Ik zou het zo weer doen!

Dan nu mijn nieuwe collega's uit het Maxima Medisch Centrum. Ik had me geen leukere start van mijn nieuwe baan kunnen bedenken dan MMSchnee!! Nog zonder een dag gewerkt te hebben mocht ik al mee op ski-uitje. Dit maakt een kennismaking wel erg leuk! Ik wil alle kinderartsen en neonatologen uit het MMC Veldhoven heel erg bedanken voor de mogelijkheid die jullie mij gegeven hebben om dokter te worden. Na 3,5 jaar fulltime onderzoek te hebben gedaan, was het voor mij zeker geen makkelijke start. Maar ieder van jullie gaf mij het vertrouwen en het 
veilige gevoel dat als beginnende dokter nodig hebt en helpt om je hoofd boven water te houden. Maar ook als ik even kopje onder ging hielpen jullie mij er weer bovenop. Jullie vertrouwen in mij als dokter hebben mij helpen groeien en uiteindelijk ook zelf het vertrouwen gegeven dat ik een goede dokter ga worden. Het is moeilijk in woorden te omschrijven hoeveel dat voor mij betekent heeft! Een kort extra bedankje voor onze begeleiders/opleiders Titia en Feico voor jullie begeleiding die mij heeft helpen groeien maar ook het geduld en de ruimte die jullie mij gegeven hebben om naast de kliniek mijn onderzoek af te kunnen ronden. En ook zeker een extra dankjewel aan mijn twee mentoren Koen en Bas voor jullie oprechte interesse, motiverende peptalks en adviezen die jullie mij gegeven hebben om mij te helpen mijn keuzes te maken en drempels te overwinnen. Koen voor jou ook veel succes met je onderzoek! Bas, niemand minder dan jij had begrip over hoe zwaar het met momenten was om mijn promotie af te ronden, bedankt voor de peptalks. Ook voor Ellen en Albertine heel veel succes met de laatste loodjes in het afronden van jullie proefschrift! En zoals beloofd, Inge, bedankt voor de chocoladereep wanneer ik er even doorheen zat, de gouden tip van de koffietjes bij B\&D en onze hardloopavonturen! Leuk dat ik een dagje mee mocht lopen op het revalidatiecentrum, jammer dat de patienten juist die dag niet kwamen opdagen ;).

Daarnaast een speciaal bedankje voor de verpleegkundig specialisten van het MMC. Van de NICU, Christ-jan, Petra, Heidi \& Danielle, wat heb ik ontzettend veel van jullie geleerd! Bedankt dat jullie iedere keer opnieuw het geduld opbrengen om ons beginnende stuntelende assistenten wegwijs te maken op deze bijzondere afdeling, het blijft een wonderlijk mooie wereld. Deze patiënten kunnen enorm blij zijn dat jullie met jullie jarenlange ervaring en deskundigheid iedere dag weer voor ze klaar staan en zulke goede zorg leveren. En dan Frank natuurlijk niet te vergeten. Duizendmaal dank dat jij mij tijdens mijn sollicitatie het voordeel van de twijfel hebt gegeven. En daarna bedankt dat je me bijna arbeidsongeschikt hebt gemaakt tijdens een mountainbike ritje door Best ;). Maar even serieus, opboksen tegen ouders en patiënten die telkens vragen of dokter Frank er is, is natuurlijk een onmogelijke taak. Dat bewijst maar hoe goed jij bent in wat je doet en dat je voor mij een groot voorbeeld bent in het vak. Ga daar vooral mee door!

Alle (ex) collega-assistenten uit het MMC - Giel, Etienne, Angel, Fia, Najla, Eveline, Richelle, Jonna, Anouk, Laurien, Lisan, Mireille, Ingrid, 
Joelle, Bianca, Mark, Kimberley, Myrthe, Noortje, Femke, Hilde - bedankt voor de hulp tijdens mijn (in)werkperiode, de gezelligheid, de TGIF-lunches, het fijne teamwork, koffiemomentjes, de etentjes en natuurlijk de ski-tripjes niet te vergeten. Jullie hebben mijn tijd in het MMC mede zo fijn gemaakt. Ik wens ieder van jullie heel veel succes met waar jullie pad ook heen mag gaan.

Ook een speciaal bedankje aan de fantastische teams van verpleegkundigen van de kinderafdeling, medium care en NICU! Zonder jullie jarenlange ervaring en vertrouwen in mij had ik nooit mijn eerste (en ook niet mijn laatste) diensten overleefd. Bedankt dat jullie mij het vertrouwen als dokter hebben gegeven. Jullie zijn ge-wel-dig! Daarnaast waren mijn vele diensten (want overdag zagen jullie mij nooit) nooit zo leuk geweest zonder vele van jullie, bedankt voor het vele lachen, de goede gesprekken en de traktaties als 'Smoskes' waar we de nacht weer mee door kwamen.

Vrienden en familie. Ondanks dat ik jullie erg heb verwaarloosd de afgelopen jaren zijn jullie wel zeker de belangrijkste mensen in mijn leven. Ik denk dat mijn dankbaarheid in woorden uit drukken hier bijna onmogelijk is en slechts een aaneenschakeling van understatements gaat zijn. Daarom hoop ik ook dat ieder van jullie wel weet wat je voor mij betekent. Ik ga het hier dan ook niet te lang maken aangezien dat misschien teniet zal doen aan hoe ik echt over jullie denk en hoe blij ik met jullie ben in mijn leven.

My old time favorites, Bo, Chanou, Karlijn, Manon, Pascalle en Sanne, al bijna meer dan de helft van ons leven zijn wij vriendinnen. Ondanks dat ik de afgelopen jaren vaker gestrest, moe en niet altijd even gezellig ben geweest zijn jullie er geweest om mij door deze periode heen te helpen. Ook al zien we elkaar wat minder vaak, heb ik het idee dat de kwaliteit er juist op vooruit gaat. Tijdens onze uitjes, jaarlijkse weekendjes weg en ski-tripjes blinken wij nog steeds uit in doen alsof we nooit ouder zullen worden! Alle zorgen worden vergeten en we weten als geen ander alles te relativeren, geen zinnig woord uit te brengen en buikpijn te hebben van de slappe lach. Bedankt dat wij er altijd voor elkaar zijn, ik hou van jullie!

Liefste Sharon, Ginger, Sanne, Brenna, Linda, Mahnaz, Suus, Sharon, Romy - Van wijn \& spelletjes avonden, weekendjes weg, festivals tot heerlijk afblazen tijdens voetbaltoernooitjes welke gevierd werden tijdens de derde helft. Ontelbaar mooie herinneringen heb ik met jullie, en nog 
veel meer gaan er zeker weten komen. Ik ben super blij met jullie in mijn leven!

Vronie, wetende dat complimentjes uitdelen in Donkiesjot uitgroeien tot zo een goede vriendschap zouden we dit veel vaker moeten doen. Samen kunnen wij goed lachen, vooral met een grote mate aan zelfspot. In combinatie met bourgondische gezelligheid, lekker eten en goede wijn maakt het samenzijn met jou altijd fijn!

Mijn mede-groepje 14 maatjes, Nadia, Nienke, Fleur \& Anouk. Samen hebben wij onze eerste stapjes in het dokter worden gezet en dat blijft voor altijd speciaal! Nadia dat ik de meest bijzondere dag uit jouw leven aanwezig mocht zijn vond ik heel bijzonder, wat een sprookjesvoorbeeld van de liefde!

Mieke, samen zij-instromen in Maastricht, onze sporadische stap-avonturen in Maastricht met bijzondere ontmoetingen, stiekem sigaretjes roken met de handdoek in ons haar, wilde fietsplannen van Maastricht naar Groningen vroegtijdig op de eerste hulp belanden, vervolgens alleen nog maar veilig met de trein en uitstappen waar we nog nooit geweest zijn, in Göteborg Daan opzoeken of een weekendje midden Nederland in de spa - het maakt niet uit wat we doen, alles is gezellig met jou! Jij wordt een echte Top-Dokter!

Lieve Elke, lief en leed hebben wij samen gedeeld gedurende onze onderzoeksjaren. Jij hebt een enorm sterk doorzettingsvermogen waardoor ik zeker weet dat jij er wel gaat komen. Hou nog heel even vol, ik gun je het beste in de wereld!

Lida, ik heb je ontmoet tijdens de MMC-introductiedag en er was meteen een enorme klik, alsof ik je al jaren ken! Ik heb bewondering voor je enorme gedrevenheid en ambities, je positieve instelling en je enorme dosis humor. Want ja, tussen de lege ketchupflessen zou ik ook nooit gelukkig worden! Ik mis je in het MMC en Eindhoven, maar wens je super veel succes in Utrecht.

Esther, mijn maatje bij TCM en ook in het MMC. Je laat me niet alleen peentjes zweten aan de andere kant van het gordijntje als die baby er weer eens niet gemakkelijk uit wilde komen maar je laat me ook afzien tijdens een fietstocht in Ibiza. Ik vond het leuk met jou! 
Dan mijn familie, klein maar heel fijn!

Mijn oom, tante, neefjes, nichtjes en aanhangsels. Hilde en Harry, bedankt dat jullie er altijd voor mij zijn geweest en ook altijd voor mij zullen zijn. Mijn onderzoekende vermogen en 'buiten de box'- denken werd als kind al enorm gestimuleerd bij jullie op de boerderij! Wat was dit altijd fijn! En ook nu nog kom ik graag bij jullie over de vloer voor een gezellig kopje koffie, een goed gesprek of een fijne knuffel! En ook Vera, Trudy, Bas \& Mart ik hou van jullie! Al lopen we de deur niet plat bij elkaar, het samenzijn is altijd fijn!

Mijn allerliefste zussen, Anke en Lieke, wij zijn al van jongs af aan zo verschillend, maar toch kan ik me een leven zonder jullie niet voorstellen. Ik ben ontzettend gek op de twee liefste kleine neefjes die jullie in de familie hebben gebracht. Ik ben super trots op jullie! Sil, wat is samenwonen met jou fijn in Eindhoven. Ons 'kleine' broertje wat ben ik trots op jou, je bent de liefste! De schoonbroers/zusje, Niels, Wilco en Mirthe, inmiddels zijn jullie al zo ingeburgerd in de familie, en ik hoop ook dat jullie dat voor altijd zullen blijven!

Liefste pap en mam, een hele grote dank jullie wel voor alles! Jullie hebben me de beste opvoeding gegeven die ik me kon wensen. Ik heb een fantastischejeugd gehad en de vrijheid gekregen om de wereld te ontdekken op mijn manier. Mijn eigen keuzes heb ik mogen maken en ook mijn fouten waarmee ik jullie behoorlijk heb uitgedaagd en jullie geduld op de proef heb gesteld. Ondanks dat heb ik mij altijd onvoorwaardelijk gesteund en geliefd gevoeld door jullie! Pap, bedankt voor het helpen ontwerpen van dit boekje! Raad eens hoeveel ik van jullie hou? 


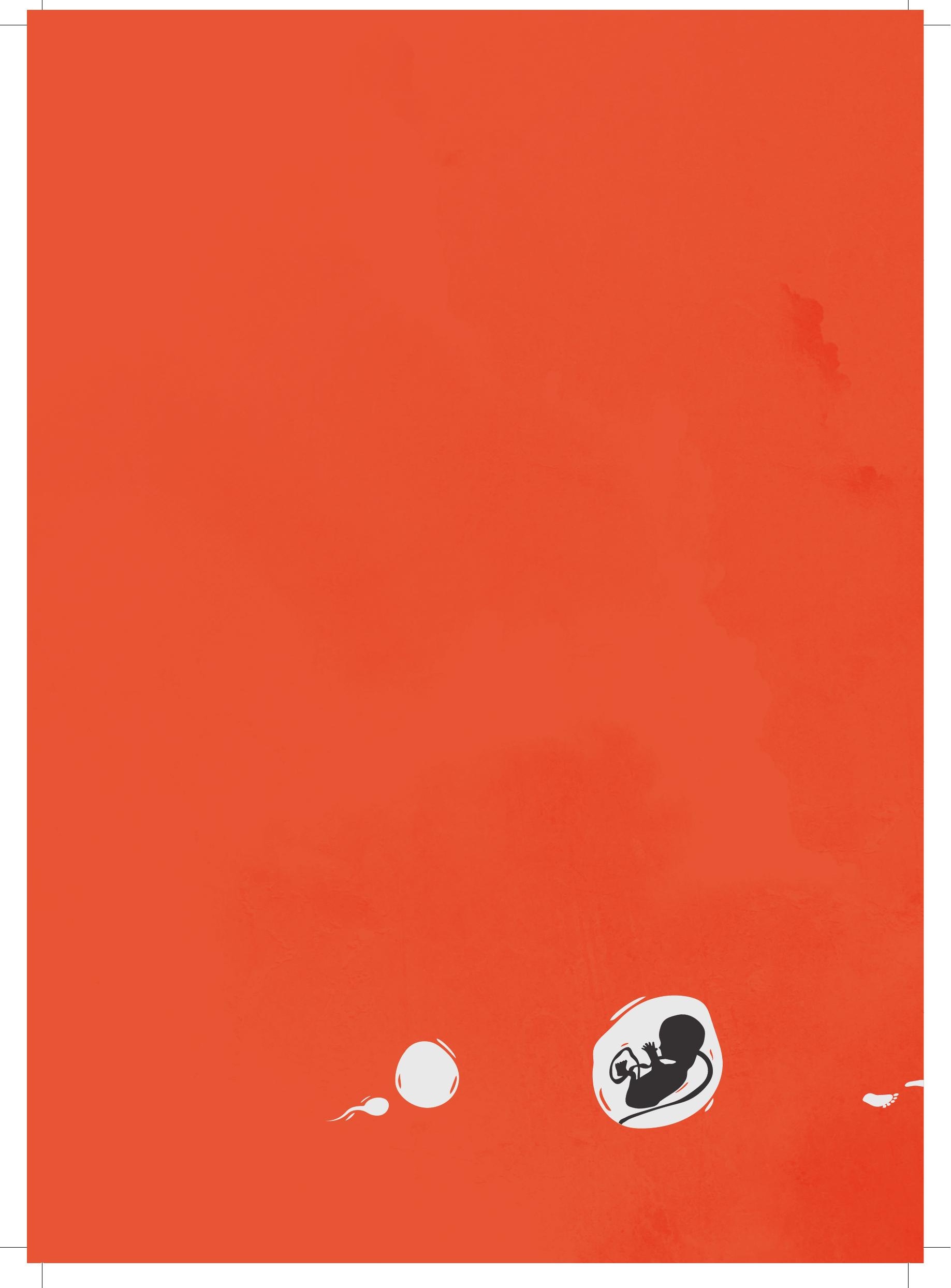




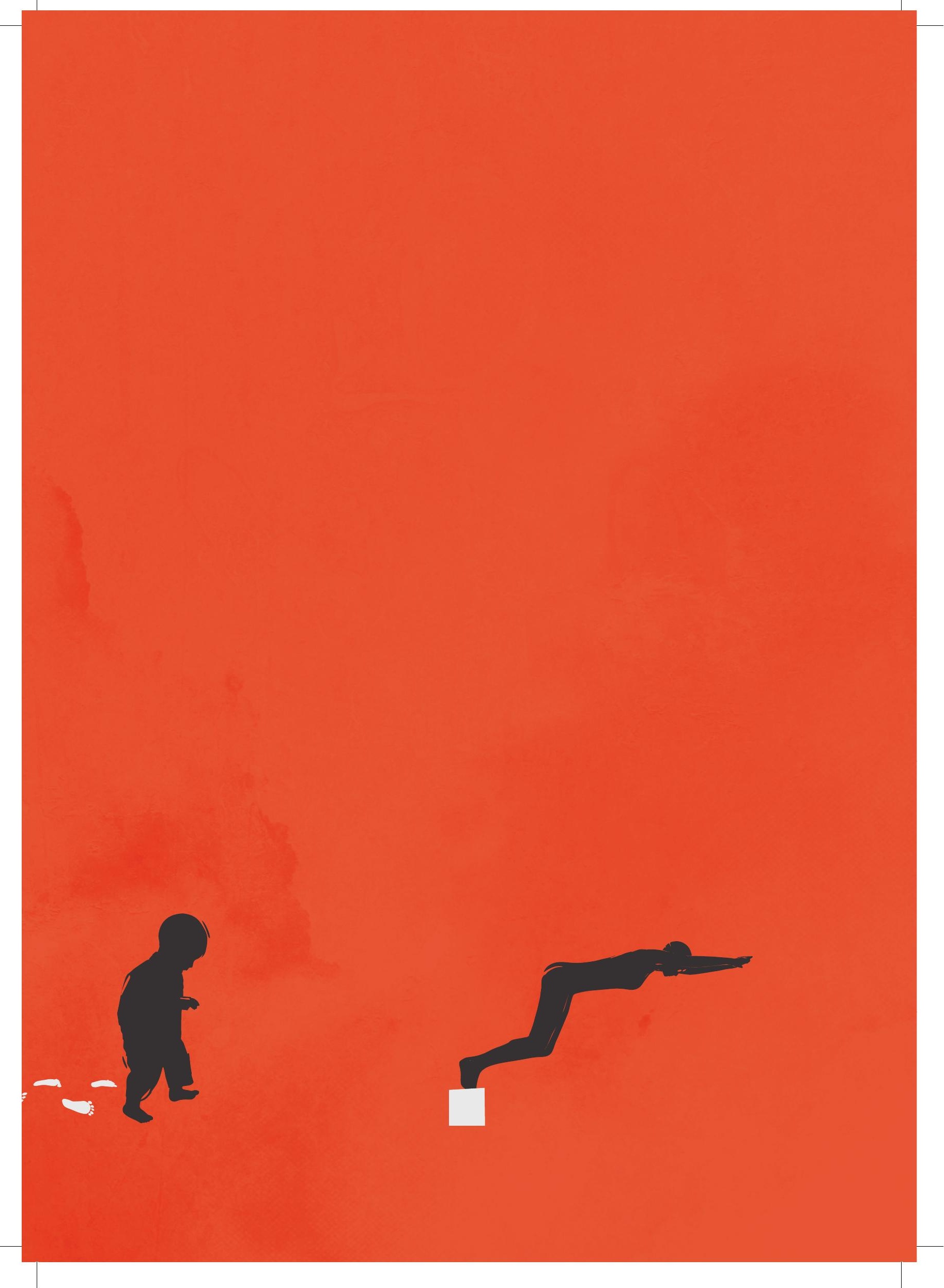


